\title{
Exploring the baroreceptor reflex function in neonates
}

Citation for published version (APA):

Andriessen, P. (2004). Exploring the baroreceptor reflex function in neonates. [Doctoral Thesis, Maastricht University]. Universiteit Maastricht. https://doi.org/10.26481/dis.20041208pa

Document status and date:

Published: 01/01/2004

DOI:

10.26481/dis.20041208pa

Document Version:

Publisher's PDF, also known as Version of record

\section{Please check the document version of this publication:}

- A submitted manuscript is the version of the article upon submission and before peer-review. There can be important differences between the submitted version and the official published version of record.

People interested in the research are advised to contact the author for the final version of the publication, or visit the DOI to the publisher's website.

- The final author version and the galley proof are versions of the publication after peer review.

- The final published version features the final layout of the paper including the volume, issue and page numbers.

Link to publication

\footnotetext{
General rights rights.

- You may freely distribute the URL identifying the publication in the public portal. please follow below link for the End User Agreement:

www.umlib.nl/taverne-license

Take down policy

If you believe that this document breaches copyright please contact us at:

repository@maastrichtuniversity.nl

providing details and we will investigate your claim.
}

Copyright and moral rights for the publications made accessible in the public portal are retained by the authors and/or other copyright owners and it is a condition of accessing publications that users recognise and abide by the legal requirements associated with these

- Users may download and print one copy of any publication from the public portal for the purpose of private study or research.

- You may not further distribute the material or use it for any profit-making activity or commercial gain

If the publication is distributed under the terms of Article $25 \mathrm{fa}$ of the Dutch Copyright Act, indicated by the "Taverne" license above, 


\title{
Exploring the baroreceptor reflex function in neonates
}

\author{
Peter Andriessen
}


Cover: Finn (In the hands of the Father, photo: Peter Andriessen ${ }^{\circledR}$ )

(C) Peter Andriessen, 2004

Exploring the baroreceptor reflex function in neonates.

Maastricht University, The Netherlands

ISBN no: $90-76014-10-8$

NUR no: 883

No part of this thesis may be reproduced or transmitted in any form or by any means, electronic or mechanical, including photocopy, recording, or any information storage and retrieval system, without permission of the copyright owner.

Lay-out: Henk en Lars Dinnissen

Printed by: Verhagen Grafische Media, Veldhoven, The Netherlands 


\section{Exploring the baroreceptor reflex function in neonates}

\section{PROEFSCHRIFT}

ter verkrijging van de graad van doctor

aan de Universiteit Maastricht,

op gezag van de Rector Magnificus,

Prof. mr. G.P.M.F. Mols,

volgens het besluit van het College van Decanen,

in het openbaar te verdedigen

op woensdag 8 december 2004 om 14:00 uur

door

Peter Andriessen 


\section{PROMOTOR:}

Prof. dr. C.E. Blanco

\section{COPROMOTOR:}

Dr. S. Bambang Oetomo (Máxima Medisch Centrum, Veldhoven)

\section{BEOORDELINGSCOMMISSIE:}

Prof. dr. R.A.M.G. Donckerwolcke (voorzitter)

Prof. dr. M. Allessie

Prof. dr. F. van Bel (Universitair Medisch Centrum Utrecht)

Prof. dr. J. de Haan

Prof. dr. M.A. Hanson (University of Southampton, Verenigd Koninkrijk)

Financial support by Abbott B.V. (Hoofddorp, The Netherlands), Dräger Medical Netherlands B.V. (Zoetermeer, The Netherlands), Friso Kindervoeding (Leeuwarden, The Netherlands), GE Healthcare Technologies (Hoevelaken, The Netherlands) and Stichting Wetenschapsfonds Máxima Medisch Centrum (Veldhoven, The Netherlands) for the reprint of this thesis is gratefully acknowledged. 


\section{Contents}

$\begin{array}{lll}\text { Chapter } 1 \text { Outline and goals of the thesis } & 7\end{array}$

Chapter 2 The baroreceptor reflex: short-term blood pressure control 11

2.1 The autonomic nervous system in reflex control of blood pressure $\quad 12$

2.2 The baroreceptor reflex as a closed loop control system $\quad 15$

2.3 Baroreceptor reflex mediated heart rate control $\quad 19$

2.4 Methods exploring baroreceptor reflex mediated heart rate control in neonates 21

2.5 Ontogeny of the baroreceptor reflex 22

2.6 Summary 25

Chapter 3 System network, acquisition and analysis of cardiovascular fluctuations $\quad 29$

3.1 System network and environment 30

3.2 Data acquisition and subjects 31

3.3 The rationale for frequency domain analysis 33

3.4 Frequency ranges of interest 34

3.5 Fast Fourier transform 35

$\begin{array}{ll}3.6 \text { Data analysis } & 37\end{array}$

3.7 Transfer function analysis $\quad 40$

3.8 Summary 43

Chapter 4 Customized spectral band analysis compared with conventional Fourier analysis of heart rate variability in neonates (Physiological Measurements 2004)

Chapter 5 Noninvasive assessment of blood pressure variability in preterm infants (Pediatric Research 2004)

Chapter 6 Cardiovascular fluctuations and transfer function analysis in stable preterm infants (Pediatric Research 2003)

Chapter 7 Cardiovascular autonomic regulation in preterm infants: the effect of atropine (Pediatric Research - In Press) 
Chapter 8 Baroreceptor reflex sensitivity in neonates: the effect of gestational age (submitted)

Chapter 9 General discussion and perspectives

9.1 Methodology

9.2 The baroreceptor reflex mediated cardiovascular fluctuations in preterm infants

9.3 Autonomic nervous control of the baroreceptor reflex

9.4 Development of the baroreceptor reflex mediated heart rate response

9.5 Perspectives: fetal programming and future research

Chapter 10 Samenvatting

Appendix A Constructing a heart rate and blood pressure variability analysis

Appendix B Feasibility of continuous noninvasive blood pressure Measurements in neonates

Appendix C Passive head-up tilting in neonates

Dankwoord 


\section{Chapter 1}

\section{Outline and goals of the thesis}


The baroreceptor reflex buffers sudden changes in systemic blood pressure by adapting heart rate and peripheral vascular resistance. Heart rate responses are mediated by both parasympathetic and sympathetic efferent nerve activity, whereas vascular resistance is adapted by sympathetic nerves only. Because vascular resistance is difficult to measure autonomic changes related to baroreceptor reflex control are usually studied by evaluating heart rate and blood pressure fluctuations only. Spectral analysis offers the opportunity to decompose spontaneously occurring fluctuations in blood pressure and heart rate into a power spectrum, and to relate the character of the fluctuations to physiological processes. Low frequency cardiovascular fluctuations of approximately $0.1 \mathrm{~Hz}$ in human adults are ascribed to the baroreceptor reflex activity and presumed to be under sympathetic and parasympathetic control, whereas high frequent fluctuations are associated with respiratory activity (respiratory sinus arrhythmia) and are under parasympathetic control only ${ }^{1}$. Standard methods of measurements and spectral band definitions have been formulated for human adults ${ }^{2}$. As the neonatal heart rate, blood pressure values and respiration rates differ from that of the adult, neonatal studies likely require different spectral band definitions. Despite abundant reports about heart rate variability in neonates 3.4 , very little information is known about blood pressure variability and notably about the interaction between heart rate and blood pressure fluctuations and underlying regulation mechanisms. In the human neonate, autonomic control of the circulation has been assessed most often by measuring alterations in heart rate in response to postural changes. Several studies have demonstrated in healthy preterm and term infants that head-up tilting (to unload the arterial baroreceptors) produce a significant heart rate response ${ }^{5-8}$. In contrast, other investigators have been unable to demonstrate a consistent response of heart rate to passive head-up tilting and concluded that the baroreceptor mediated heart rate response is poorly developed during the neonatal period 9.10 .

Because little is known in neonates about short-term regulation of blood pressure, the autonomic nervous system and how regulation is affected by perinatal factors I was interested in exploring the baroreceptor reflex by measuring beat-to-beat signals of heart rate and arterial blood pressure. Advantage was taken of standard neonatal intensive care management (invasive arterial catheters, monitoring and atropine administration) in clinical recovering infants, ranging from very preterm to term gestation. 
In addition, the interest in baroreceptor reflex function in the neonate was driven by the association between low birth weight, increased sympathetic activity in the perinatal period and subsequent high blood pressure in adulthood, found in several experimental animal and human studies ${ }^{11-14}$.

\section{Goals of the thesis}

1. To develop a methodology for analyzing heart rate and blood pressure signals in neonates.

2. To study the baroreceptor reflex mediated cardiovascular fluctuations by transfer function analysis.

3. To study the role of the autonomic nervous system on the baroreceptor reflex.

4. To study the development of baroreceptor reflex function with age.

5. To formulate perspectives related to 'fetal programming' of the baroreceptor reflex.

A short overview of the baroreceptor reflex is discussed in chapter 2. Data acquisition and variability analysis is presented in chapter 3 . In chapter 4 a customized filtering technique is presented and compared with fast Fourier transform for analyzing shortterm variability. A method for noninvasive assessment of blood pressure signals is presented in chapter 5 . The existence of a functioning baroreceptor reflex in stable very preterm infants is presented in chapter 6 . In chapter 7 a study is presented in which atropine modulates heart rate and blood pressure variability in very preterm infants, suggesting that baroreceptor mediated parasympathetic control of heart rate is of significance for cardiovascular control. The effect of gestational age on baroreceptor reflex functioning is presented in chapter 8 . Finally, a general discussion with conclusions and future perspectives are discussed in chapter 9. 


\section{References}

1. Akselrod S, Gordon D, Madwed JB, Snidman NC, Shannon DC, Cohen RJ. Hemodynamic regulation: investigation by spectral analysis. Am J Physiol 1985;249:H867-H875

2. Task Force of the European Society of Cardiology and the North American Society of pacing and electrophysiology. Heart rate variability: standards of measurement, physiological interpretation, and clinical use. Circulation 1996;93:1043-1065

3 Rosenstock EG, Cassuto Y, Zmora E. Heart rate variability in the neonate and infant: analytical methods, physiological and clinical observations. Acta Paediatr 1999;88:477-82

4 Vălimäki I, Rantonen T. Spectral analysis of heart rate and blood pressure variability. Clinics in Perinatol 1999;26:967-980

5 Picton-Warlow CG Mayer FE. Cardiovascular responses to postural changes in the neonate. Arch Dis Child 1970:45:354-359

6 Finley JP, Hamilton R, MacKenzie MG. Heart rate response to tilting in newborns in quiet and active sleep. Biol Neonate 1984:45:1-1

7 Thoresen M, Cowan F, Walloe L. Cardiovascular responses to tilting in healthy newborn babies. Early Human Dev 1991;26:213-222

8 Chen CM, Tsai TC, Lan MC. Effect of body tilting on physiological functions in healthy term neonates. Acta Paediatr 1995;84:474-477

9. Waldman S, Krauss AN, Auld PAM. Baroreceptors in preterm infants: their relationship to maturity and disease, Develop Med Child Neurol 1979; 21:714-722

10. Holden K, Morgan JS, Krauss AN, Auld PAM. Incomplete baroreceptor responses in newborn infants. Am J Perinatol 1985;2:21-34

11. Phillips DIW, Barker DJP. Association between low birth weight and high resting pulse in adult life: is the sympathetic nervous system involved in programming the insulin resistance syndrome? Diabet Med 1997; 14:673-7

12. Jansson T, Lambert GW. Effect of intrauterine growth restriction on blood pressure, glucose intolerance and sympathetic nervous system activity in the rat at 3-4 months of age. J Hypertens 1999; 17:1239-1248

13. Ruijtenbeek K, le Noble FAC, Janssen GMJ, Kessels CGA. Fazzi GE, Blanco CE, de Mey JGR. Chronic hypoxia stimulates periarterial sympathetic nerve development in the chick embryo. Circulation 2000;102:2892-2897

14. IJzerman RG, Stehouwer CDA, de Geus EJ, van Weissenbruch MM, Delemarre-van de Waal HA, Boomsma DI. Low birth weight is associated with increased sympathetic activity. Circulation 2003;108:566-571 


\section{Chapter 2}

\section{The baroreceptor reflex:}

short-term blood pressure control 


\subsection{The autonomic nervous system in reflex control of blood pressure}

The importance of the baroreceptor reflex is to stabilize perfusion pressure in the face of disturbances of circulatory homeostasis. Short-term control of blood pressure is achieved by regulatory adjustments initiated by a change in the pressure load at specialized pressure sensors located at the aortic arch and the carotid sinuses. The baroreceptor reflex arch consists of baroreceptors, afferent nerves, central nervous nervous system, and efferent nerves ${ }^{1}$.

Baroreceptors in the aortic and the carotid vasculature are mechanoreceptors and are almost exclusively located in the adventitia of these arteries ${ }^{2}$. When arterial pressure increases, there is an increase in the activity of baroreceptor afferents, which travel centrally to the nucleus of the solitary tract in the medulla ${ }^{3,4}$. This signal ultimately results in an increase in vagal cardioinhibitory activity and a decrease in sympathetic discharge, which act to reduce heart rate and vascular resistance buffering the increase in arterial pressure. Conversely, a fall in arterial pressure produces a decrease in baroreceptor afferent discharge, resulting in alterations in autonomic activity to increase arterial pressure. In this manner, the arterial baroreceptor reflex functions as a negativefeedback control system for short-term regulation of cardiovascular function.

The central integration of reflex inputs occurs in part at the level of the nucleus of the solitary tract ${ }^{3}$. The nucleus of the solitary tract is located in the medial area of the medulla oblongata and is important because of its multiple afferent connections. The nucleus of the solitary tract contains a medullary inhibitory (depressor) region, which either activates parasympathetic or inhibits sympathetic efferent pathways. Its major efferent pathways activate vagal neurons in the nucleus ambiguous and the dorsal motor nucleus. Cardiac vagal neurons in the nucleus ambiguous and the dorsal motor nucleus activate cholinergic vagal pathways to the heart. In addition nucleus of the solitary tract efferent pathways inhibit preganglionic sympathetic neurons. By contrast, the lateral area of the medulla contains the pressor region, where the rostral ventrolateral medulla is the main output site for baroreceptor reflex modulation of efferent sympathetic nerve activity. The rostral ventrolateral medulla receives input from a variety of central nervous system areas directly or indirectly linked to baroreceptor reflex function, such as the nucleus of the solitary tract or hypothalamus. 
The parasympathetic system consists of relatively long preganglionic neurons with somata originated in the nucleus ambiguous and the dorsal motor nucleus of the medulla oblongata that project axons into the nervi vagi and synapse on postganglionic local neuronal circuits adjacent to the heart. By contrast, the sympathetic system consists of preganglionic neurons with somata originated in the spinal cord that project axons into the rami communicans to synapse on relatively long intrathoracic sympathetic efferent postganglionic neurons that innervate the heart and circulation. Figure 1 shows a simplified diagram of these neuronal pathways that may be involved in cardiopulmonary regulation 5 .

Sympathetic nervous control of vessel diameter occurs via binding of epinephrine or norepinephrine to postganglionic $\alpha$ - and $\beta$-adrenergic receptors. Stimulation of $\alpha$ - and $\beta 2$-receptors results in vasoconstriction and vasodilatation, respectively. Stimulation of the cardiac $\beta 1$-receptors results in increase of heart rate and contractility. Parasympathetic effects occur predominantly through binding of acetylcholine to postganglionic muscarinic receptors of the sino-atrial node and atrioventricular node of the heart. Binding of acetylcholine to muscarinic receptors leads to a rapid (within 100 ms) membrane hyperpolarization resulting in decrease of heart rate ${ }^{6}$. In addition, the effect of vagal impulse is brief because acetylcholine is rapidly hydrolyzed ${ }^{6}$. Both characteristics of acetylcholine enable the parasympathetic system to be a fast-acting system. By contrast, cardiac responses to sympathetic stimulation arise relatively slowly (latency of 2-5 seconds) and dissipate over a longer period of time. This slow sympathetic response is caused by a slow dispersion of norepinephrine following release at sympathetic nerve endings, slow re-uptake into sympathetic nerve terminals, and complex cell reaction leading to a long response time to sympathetic stimulation ${ }^{6,7}$.

In summary, the physiological implications of the neuronal architecture are that rapid onset and offset of cardiac vagal responses allow for beat-to-beat parasympathetic regulation of heart rate, whereas the slow temporal response to sympathetic stimulation precludes any such dynamic regulation. For this reason it is assumed that faster (respiratory associated) fluctuations are associated with the parasympathetic system, whereas slower fluctuations are attributed to the baroreceptor reflex and related to predominant sympathetic and parasympathetic activity ${ }^{8}$. 


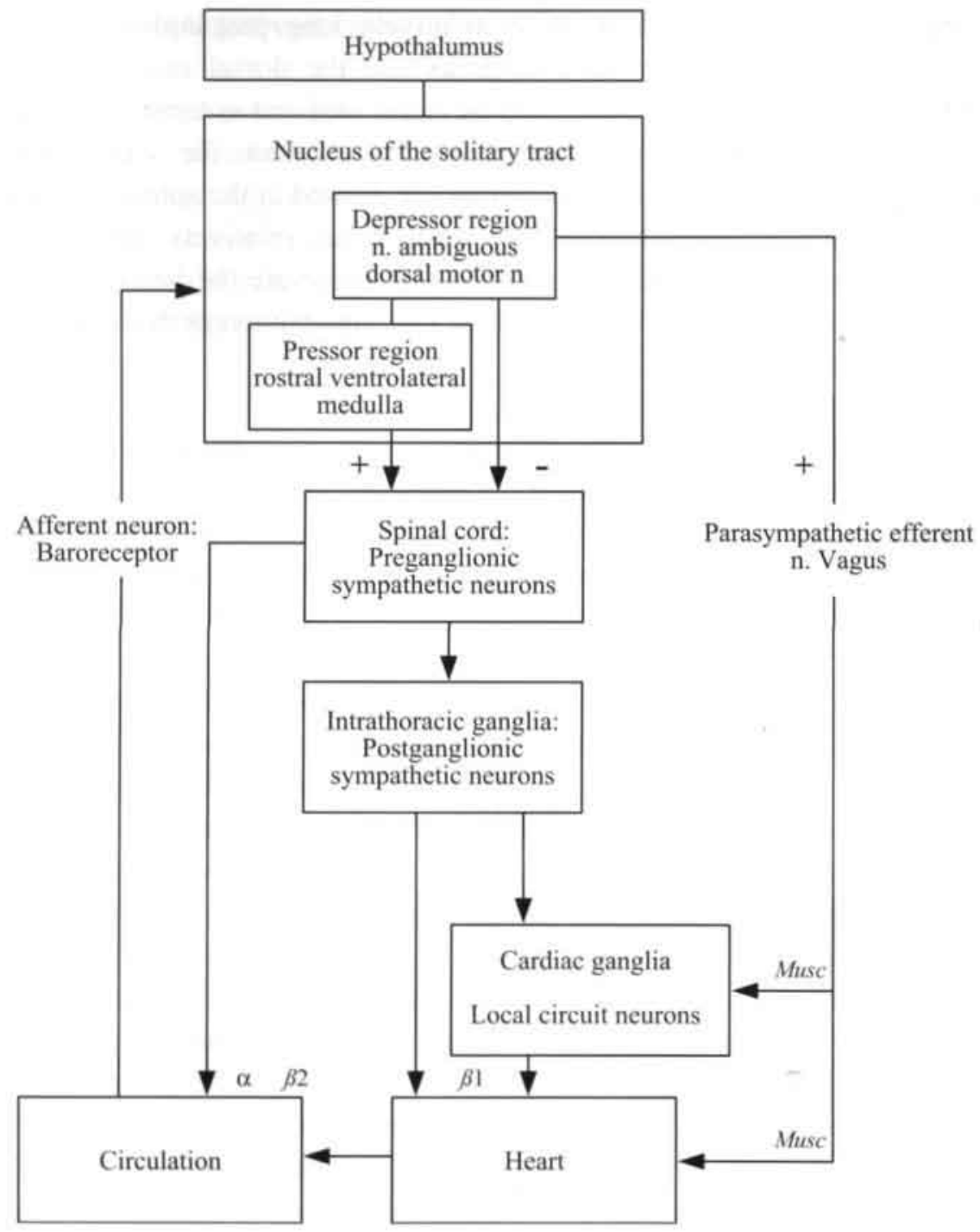

Figure 1. A simplified diagram of the neuronal circuits that are involved in cardiovascular regulation. Afferent axons arising from the baroreceptors in the aortic and carotid arteries connect with neurons in the medulla oblongata. The central integration of reflex inputs occurs at the level of the nucleus of the solitary tract. The nucleus of the solitary tract contains a medullary inhibitory depressor region (nucleus ambiguous, dorsal motor nucleus) which either activates parasympathetic (+) or inhibits (-) sympathetic efferent pathways. The lateral area of the medulla contains the pressor region (rostral ventrolateral medulla) which activates $(+)$ efferent sympathetic activity. Efferent preganglionic sympathetic neurons interact with efferent sympathetic postganglionic and local circuit neurons. Sympathetic nervous control of vessel diameter is mediated via $\alpha$ - (vasoconstriction) and $\beta 2$ - (vasodilatation) receptors. Stimulation of $\beta 1$ receptors results in increase of heart rate and contractility. The parasympathetic effects occur through binding of acetylcholine to muscarine (Musc) receptors. 


\subsection{The baroreceptor reflex as a closed loop control system}

System engineers have developed a set of mathematical methods collectively called control or system theory, which provide tools for quantitative analyses of various physiological control systems ${ }^{9}$. The arterial baroreceptor reflex system is one of the systems to which such analyses were applied very early in the cross-fertilization of physiology and control theory ${ }^{10.11}$.

In terms of system analysis the arterial baroreceptor reflex is a negative-feedback control system because an increase in arterial pressure increases the baroreceptor afferent impulses, which in turn decrease the arterial pressure through the reflex arc. A general diagram of a negative-feedback control system is given in Figure $2^{1,12}$.

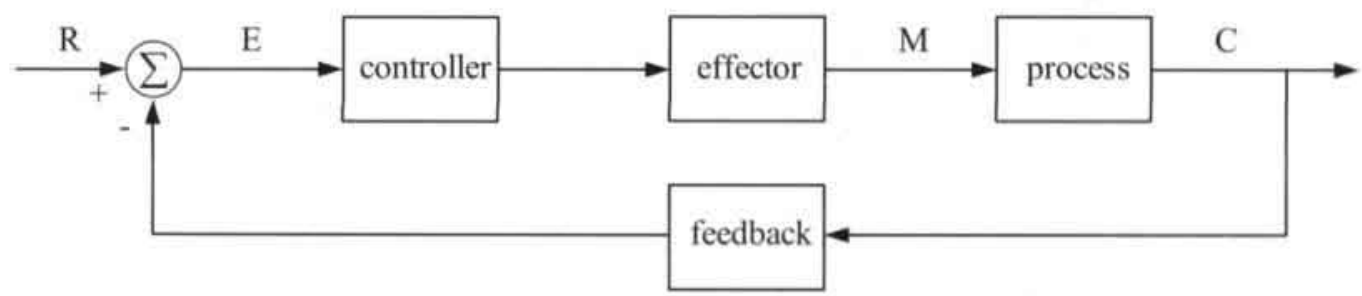

Figure 2. General diagram of a negative-feedback control system. C is the controlled variable, R the reference input, $\mathrm{E}$ the error of the signal and $\mathrm{M}$ the effector output.

The output variable $\mathrm{C}$ is to be controlled (arterial blood pressure) and is continuously measured by a feedback sensor (aortic and carotid sinus baroreceptors), which provides afferent information for the controller. The autonomic nervous system is the controller which integrates afferent information, compares it to a pressure set-point and controls different effector mechanisms through the parasympathetic and sympathetic efferent neural innervation. The difference between the reference value $\mathrm{R}$ or set-point, determines the error signal $\mathrm{E}$, also called the driving signal for the effector. Among the effectors are the sino-atrial node, cardiac muscle contractility and the arterial and venous smooth muscle cells. The effector finally influences the controlled process by a control variable $\mathrm{M}$. The baroreceptor reflex function as a homeostatic (constant reference value) and proportional control system (the effector signal $\mathrm{M}$ is proportional to the error signal E).

Systemic arterial pressure is the product of cardiac output and total peripheral resistance. The reflex controls vascular resistance by changing the tone of smooth muscle in the wall of the vessels in the entire systemic arterial vascular network. Likewise, cardiac output is the product of heart rate and stroke volume, and the reflex 
controls cardiac output by adapting pacemaker activity and contractility of the ventricles. In addition the reflex controls diastolic filling of the ventricles by changing heart rate and by changing the total venous capacity, which determines the venous return. Taken together, there are four effectors by which the controller regulates arterial blood pressure: heart rate, venous return, contractility, and total peripheral resistance. Figure 3 shows a simplified diagram of these four effectors ${ }^{12}$. The left part of the figure represents the controller with its afferent nerves, central nervous system, and efferent nervous ( $\alpha$ - and $\beta$-sympathetic and parasympathetic system). The right part of the figure shows the controlled process influenced by these effectors. A fall in arterial pressure decreases afferent nerve activity by the baroreceptors, causing the central nervous system to increase sympathetic and decrease parasympathetic nervous activity, respectively. The collective activation of these effectors results in restoring the blood pressure fall. The picture as shown in Figure 3 is very simplified, particularly the complex role of the nervous system, which is presented as a black-box system.

The important message that control theory brought to physiology is that to best evaluate the performance of feedback control systems, the feedback loop should be opened at an appropriate site, usually where the controlled variable is seen by the feedback sensor ${ }^{1}$. In this way the static and dynamic relationships between the input and output signals can be determined. This is called open-loop analysis. If the input variable of the opened system is varied stepwise and steady-state change in the output variable is related to the input, the input-output relation curve represents the static (as opposed to dynamic) performance of the opened system over the investigated range of input magnitude.

In negative-feedback systems the input-output relation curve usually runs from the upper left to the lower right because an increase in input $(\Delta \mathrm{I})$ decreases output $(\Delta \mathrm{O})$ (Figure 4, left panel). The input-output relation curve is sigmoidal, having a threshold zone in the small input region (where output is maximal) and a saturation zone in the large input region (where output is minimal). The curve over the intermediate region may or may not be linear. The slope of the relation curve $(\Delta \mathrm{O} / \Delta \mathrm{I})$ is the static open-loop gain. Because the baroreceptor reflex input-output relation is sigmoid the gain varies with $\mathrm{I}$. Therefore it is necessary to determine a gain value $(\mathrm{G})$ around a given absolute magnitude of an input variable I. The gain curve (Figure 4, right panel) has a peak value $\left(\mathrm{G}_{\max }\right)$ and its shape resembles that of a normal distribution curve. The operating point (or set point) is the equilibrium point at which input is equal to output and does not necessarily coincide with the optimum input at which the slope of the curve is maximal. 


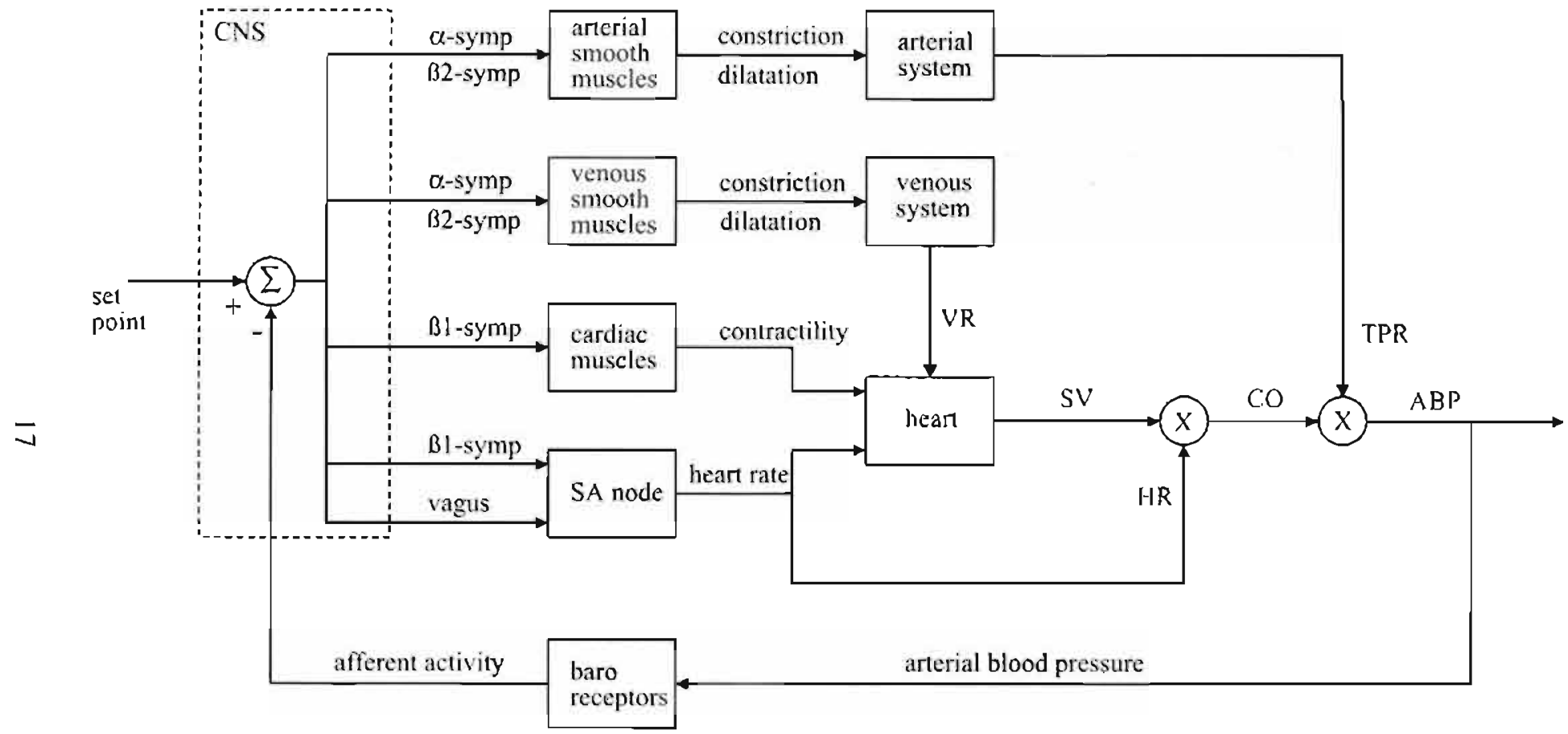

Figure 3. After: Ten Voorde 1992. The diagram shows the baroreceptor closed-loop control of systenic arterial blood pressure with negative fecdback by the baroreceptors and the central nervous system (CNS) as the controlier. Four different effectors are conirolled through the different autononic pathways, divided into $\alpha$ - and $\beta$-sympathetic and vagal system. Abbreviations: arterial blood pressure (ABP), heart rate (HR), cardiac: output (CO). stroke volume (SV), venous return (VN). tolal peripheral resistance (TPR). 

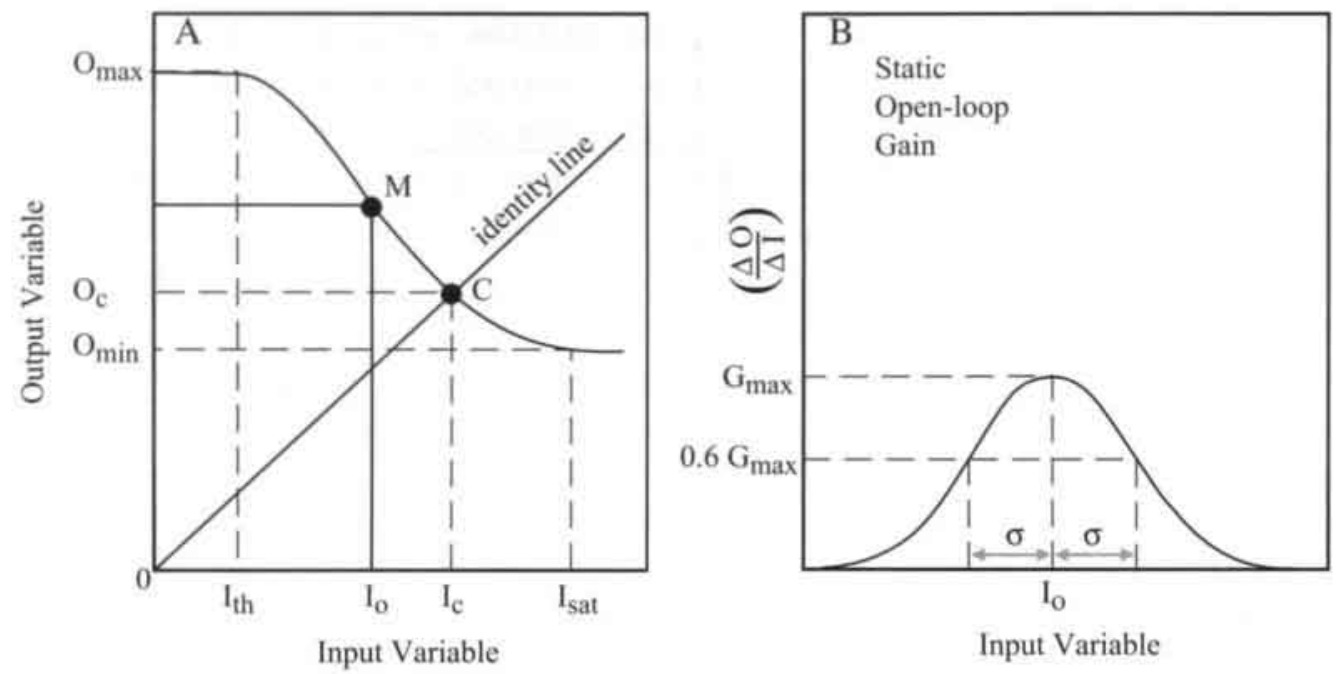

Figure 4. After: Sagawa 1983. Panel A represents an open-loop input-output relationship curve of a negative-feedback system. When the input is at threshold $\left(\mathrm{I}_{\mathrm{th}}\right)$, output is maximal $\left(\mathrm{O}_{\max }\right)$; when the input is at saturation $\left(\mathrm{I}_{\text {sat }}\right)$, output is minimal $\left(\mathrm{O}_{\min }\right)$. M, optimum input $\left(\mathrm{I}_{\mathrm{o}}\right)$ at which slope of curve (gain) is maximal; $\mathrm{C}$, operating point or closed-loop point equilibrium point (input=output). $\mathrm{M}$ and $\mathrm{C}$ may or may not coincide. Panel B shows the open-loop gain (slope of curve in $A$ ) as function of input. $G_{\max }$, maximum gain when input is optimum $\left(\mathrm{I}_{\mathrm{o}}\right), \sigma$, magnitude of deviation of $\mathrm{I}$ from $\mathrm{I}_{\mathrm{o}}\left(0.6 \mathrm{G}_{\max }\right.$. Gaussian distribution).

In clinical human research of baroreceptor reflex function, however, the operating point is often considered as the point of the reflex at which the reflex responds most effectively to changes around the physiological values of arterial pressure ${ }^{13}$. The baroreceptor reflex sensitivity (gain) is estimated from the magnitude of the reflex response per unit of arterial blood pressure deviation from the operating point.

In 1929, almost 30 years before control theory was used in physiology, Koch opened the carotid sinus reflex loop in the rabbit ${ }^{14}$. After cutting the nervus vagus to prevent counteraction from the aortic arch baroreceptor reflex, he varied carotid sinus pressure to determine its relation to the fall in mean arterial blood pressure. He called the sigmoidal stimulus-response curve die Blutdruckcharakteristik and found a threshold-, optimum- and saturation pressure for the isolated carotid sinus. It is one of the first examples in which the implications of physiological negative-feedback control were clearly recognized and its static open-loop performances quantitatively analyzed, just as technological systems are analyzed today. However, in conscious animals it is difficult to isolate and change the carotid sinus pressure, and in humans invasive experiments are not ethically justified. Therefore several methods have been developed to estimate the open-loop characteristics of the baroreceptor reflex from closed-loop experiments 
in animals and human adults. Among these perturbations to estimate arterial baroreceptor reflex gain from closed-loop properties are applying external counter pressure by neck chamber, hemorrhage and drug administration of epinephrine or nitroprusside ${ }^{15-17}$. A major disadvantage is that analysis is limited to the range of equilibrium pressure in the closed-loop condition. Major advantages are absence of surgical damage to the baroreceptor region and the naturally pulsatile forcing of the baroreceptors. With physiological pulsation in the carotid sinus the operative range of sinus carotid pressure is extended to a lower region than with non pulsatile carotid sinus pressure $^{18}$.

\subsection{Baroreceptor reflex mediated heart rate control}

Baroreceptor reflex mediated heart rate control has been studied much more than the other effector mechanisms (peripheral resistance, venous return and cardiac contractility, see Figure 3 ) because of its easy access to the signals. Therefore arterial baroreceptor reflex response of heart rate has been studied frequently by transiently increasing or decreasing arterial pressure by administering vaso-active agents ${ }^{16.19}$ or applying a positive- or negative-pressure pulse to the neck to decreased or increase the transmural pressure of the carotid sinus region ${ }^{20}$. By injecting vaso-active drugs, arterial blood pressure rises or falls from the equilibrium point (physiological arterial blood pressure), respectively, and an input-output curve may be constructed. The inputout curve is frequently presented as an arterial blood pressure - R-R interval curve (Figure 5). The input-output relation curve is sigmoidal, having a threshold zone in the lower blood pressure values (where R-R interval is minimal) and a saturation zone in the higher blood pressure values (where R-R interval is maximal).

Two parameters are often determined to estimate baroreceptor mediated heart rate control in clinical research ${ }^{13}$. First is the operating point of the reflex: at which level does the reflex respond most effectively to changes in arterial pressure. Second is the gain or baroreceptor sensitivity: the magnitude of the reflex response per unit of arterial blood pressure deviation from the operating point. The baroreceptor sensitivity is classically defined as the slope of the linear part of the sigmoidal blood pressure-heart rate relationship ( $R-R$ interval change per unit of arterial blood pressure change) plotted during the pressure rise (pressor response) or decrease (depressor response), induced by the administration of epinephrine or sodium nitroprusside, respectively ${ }^{16.21,22}$. In healthy adults, the baroreceptor sensitivity during induced arterial blood pressure rise and fall is approximately 30 and $10 \mathrm{~ms} / \mathrm{mmHg}$, respectively ${ }^{23}$. In mechanically ventilated and paralyzed human infants undergoing major surgery, the baroreceptor 
sensitivity was approximately 11 and $4 \mathrm{~ms} / \mathrm{mmHg}$ during blood pressure rise and fall, respectively ${ }^{24}$.

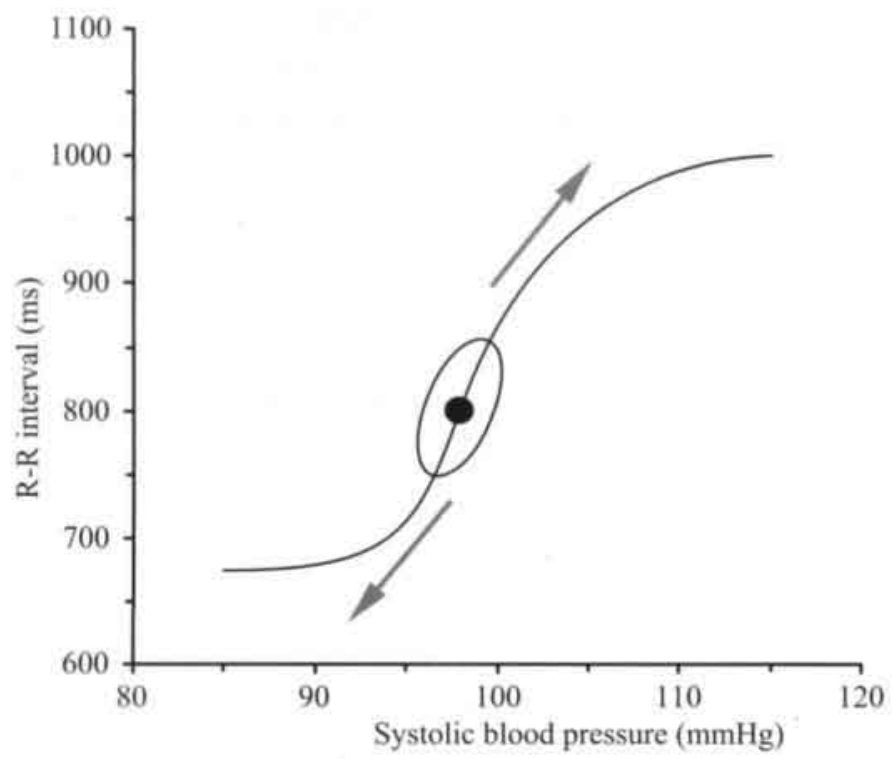

Figure 5. After: Korner 1974. The diagram represents a closed-loop relationship between arterial systolic blood pressure and R-R interval. The operating point is indicated by the dot. The blood pressure-heart rate relationship ( $\mathrm{HR}$ or R-R interval change per unit of BP change) is plotted during the pressure rise (pressor response) or decrease (depressor response), induced by the administration of epinephrine or sodium nitroprusside, respectively (indicated by the arrows). By contrast, in methods using spontaneously occurring fluctuations, the range of gain is very limited around the operating point (indicated by the oval), and thus do not reach the threshold level or saturation level.

Although these methods of studying the reflex control of heart rate are useful, the transient nature of the pressure alteration is likely to reveal only reflex control of heart rate through the parasympathetic effector limb ${ }^{25}$. It is shown in various species of animals that the parasympathetic control has almost no time lag in response to dynamic changes in arterial pressure, whereas reflex control of heart rate through the sympathetic cardiac nerves has a long time $\mathrm{lag}^{25}$. Cardiac slowing provoked by raising arterial blood pressure is mediated primarily by an increase in parasympathetic discharge, heart rate increase provoked by falling arterial blood pressure appears to be mediated by increased $\beta$-adrenergic activity, as well as reduced vagal discharge ${ }^{26}$. In normal conscious humans, reflex parasympathetic stimulation and withdrawal primarily control heart rate responses to changes in blood pressure ${ }^{26}$. Sympathetic stimulation, while influencing basal heart rate, plays a minor role in baroreceptor 
mediated heart rate control, as heart rate response to raising and falling blood pressure can be blocked by atropine and not by propanolol 21,27 . The higher baroreceptor sensitivity during blood pressure rise than during fall suggests a relative low vagal inhibitory tone during resting conditions.

In summary, the baroreceptor mediated heart rate response to increase arterial blood pressure seems to be exclusively mediated by an increase in the cardioinhibitory influence of the vagus. In addition the excitation of the sinus node that accompanies baroreceptor deactivation is also largely mediated by the vagus. Thus, although the arterial blood pressure-heart period relation curve is frequently used as a surrogate of baroreceptor sensitivity curve of the overall state of the baroreceptor reflex system, one should keep in mind that this is a very simple representation of the system.

\subsection{Methods exploring baroreceptor reflex mediated heart rate control in neonates}

The pharmacological method of altering the cardiovascular system is not always appropriate in all clinical situations and furthermore limited in neonates for medical ethical reasons. Therefore alternative methods have been developed to estimate baroreceptor function ${ }^{28}$. Among these methods are two techniques, based on the occurrence of spontaneous fluctuations in blood pressure and heart rate: 1) the sequence method and 2) cross spectral analysis or transfer function analysis between arterial blood pressure fluctuations and heart rate.

In the sequence method, recording of blood pressure and ECG are analyzed for the occurrence of sequences of spontaneously increasing or decreasing arterial pressures and the beat-by-beat effect on following cardiac intervals ${ }^{29}$. Note that with this method only arterial blood pressure-R-R interval sequences consistent with a baroreceptor reflex ( $\mathrm{R}-\mathrm{R}$ interval increase in response to increase in blood pressure and $\mathrm{R}-\mathrm{R}$ interval decrease in response to blood pressure fall) are analyzed. Hence only a part (approximately 20\%) of the total cardiac cycles is included in the analysis. In several studies this method has been validated with the drug-induced method and proved to be a reliable estimate of human cardiac baroreceptor reflex sensitivity within its physiological operating point ${ }^{23,29}$. Several investigators found baroreceptor sensitivity values, derived form this spontaneous sequence method of approximately 4 and 10 $\mathrm{ms} / \mathrm{mmHg}$ in preterm and term infants, respectively ${ }^{30.31}$. A disadvantage of the sequence method is that no clear interpretation can be given of the autonomic nervous 
involvement of the reflex. In this thesis the sequence method has not been used.

Spectral analysis offers the opportunity to decompose spontaneously occurring fluctuations in blood pressure and R-R interval series into a power spectrum, and to relate the character of the fluctuations to physiological processes. Relatively slow oscillations of arterial blood pressure with a wave length of approximately $10 \mathrm{~s}$ (or equal to $0.1 \mathrm{~Hz}$ ) in humans are often referred to as "Mayer" waves ${ }^{32}$. These low frequency fluctuations of $0.1 \mathrm{~Hz}$ are ascribed to baroreceptor activity ${ }^{8}$ and supposed to be under sympathetic and parasympathetic activity. Cross spectral (or transfer function) analysis between arterial blood pressure and R-R interval series at the low frequent band $(0.04-0.15 \mathrm{~Hz})$ has shown to be a reliable estimate of baroreceptor reflex sensitivity ${ }^{33-36}$. By contrast, relatively higher fluctuations (in adults, approximately $0.25 \mathrm{~Hz}$ ) are associated with respiratory activity and supposed to be under parasympathetic control only. In adults, the ratio of low-to-high spectral power (LF/HF ratio) is often considered to be an estimate for sympathovagal balance ${ }^{37}$. Thus, in contrast to sequence analysis, spectral analysis may be useful as a tool to determine baroreceptor sensitivity as well as 'autonomic balance'.

Because the spectral analysis method is used in several studies, the aspects of heart rate and blood pressure variability and transfer function analysis are discussed in detail in chapter three. Briefly, transfer function gain $(\mathrm{ms} / \mathrm{mmHg})$ reflects the degree to which the input signal (arterial blood pressure) amplitude becomes manifest in the output signal ( $\mathrm{R}-\mathrm{R}$ interval) amplitude at a discrete frequency. The transfer function phase (degrees or seconds) indicates the lead or lag of one signal with respect to the other at a discrete frequency.

\subsection{Ontogeny of the baroreceptor reflex}

Knowledge about the ontogeny of the autonomic cardiovascular control system and baroreceptor reflex activity relies almost exclusively on animal studies. The autonomic nervous system influences cardiovascular control during fetal development, although its contribution changes with maturation. In a sheep model, $\beta$-adrenergic blockade with propanolol of the sympathetic system has profound effects on heart rate in all fetuses regardless of gestational age ${ }^{38,39}$. On the other hand, cholinergic blockade with atropine produces no consistent effect on heart rate in preterm fetal sheep and only a slight increase in heart rate in term fetuses ${ }^{38}$. Thus, during intra-uterine life the sympathetic cardio-accelerating tone predominates over the parasympathetic cardio-inhibitory tone. After birth, however, significant changes occur in the magnitude of tones exerted on 
resting heart rate by the sympathetic and parasympathetic branches of the autonomic nervous system, respectively. The parasympathetic tone rises quickly within the first weeks of life to adult levels, while adrenergic tone decreases ${ }^{40}$. These findings suggest that basal sympathetic tone is important in the maintenance of fetal arterial blood pressure and baroreceptor reflex activity. By contrast, parasympathetic activity has little influence on basal fetal cardiovascular function except at term, while there is a progressive influence on heart rate with postnatal maturation.

The two primary species used to study vertebrate cardiovascular development, sheep and chickens, both possess a functional baroreceptor reflex during fetal or embryonic life $\mathrm{e}^{41,42}$. This suggests that either the baroreceptor reflex is an important component of cardiovascular regulation during development or that it becomes functional in anticipation of birth. Studies in embryonic chickens suggest the latter, given that the baroreceptor reflex response emerges very late in development (at approximately $90 \%$ of incubation) and have lower baroreceptor sensitivity compared with those in adults ${ }^{42}$. By contrast, fetal sheep possesses a baroreceptor reflex as early as $60 \%$ of gestation ${ }^{43}$. In addition, studies of baroreceptor activity (afferent limb of the baroreceptor reflex) in response to carotid sinus pressure, studies of renal sympathetic nerve activity in response to arterial pressure (sympathetic efferent limb) and baroreceptor mediated heart rate response (vagal efferent limb) suggest a decreasing baroreceptor sensitivity with advancing intra-uterine development in sheep $41,43,44$. After birth, baroreceptor reflex sensitivity continues to decrease as arterial rises to its resting postnatal values $^{41,44}$.

Data about the ontogeny of the baroreceptor reflex and functional maturation in the human infant are limited and conflicting 45 . This is partly caused by the limited experimental (pharmacological or mechanical) possibilities to challenge the baroreceptor reflex in neonates. Passive head-up tilt test has been applied to neonates measuring responses in blood pressure, heart rate and limb blood flow to body tilting. In preterm infants (gestational age, 26-37 wk), passive head-up tilt resulted in significant vasoconstriction of the lower limb with a slight fall in aortic blood pressure and unchanged heart rate ${ }^{46}$. The inadequate ability to maintain blood pressure and the lack of a tachycardia suggest that preterm infants lack the full integrated baroreceptor reflex response as seen in adults. In term infants, however, a fall in blood pressure was observed in conjunction with a tachycardia and a fall in limb blood flow, suggesting the presence of active reflex vasoconstriction ${ }^{47}$. Others, however, conclude that preterm as well as term babies (gestational age, 33-37 wk) show a well-developed heart rate response to passive head-up tilt ${ }^{48}$. A clear heart rate response to passive head-up tilt is present in full-term babies after 2 hours of birth and progressively increases within the 
first postnatal day ${ }^{49}$. By contrast, some other studies do not show consistent evidence of a well-developed baroreceptor reflex mediated heart rate response in the neonate 50,51 . The disparity in results may be, beside gestational age, explained by methodological differences, age at study, smoothness of tilting procedure, sleep state and the methods of measurements. Thus, the available studies of heart rate response to passive head-up tilt in human neonates suggest at least qualitatively a baroreceptor reflex mediated heart rate response is present in early postnatal life. In more recent studies the baroreceptor reflex sensitivity is estimated quantitatively from spontaneously occurring fluctuations in blood pressure and R-R interval series ${ }^{30,31}$. Change in baroreceptor reflex sensitivity is considered to be an important quantitative measure of baroreceptor reflex maturation during development ${ }^{41,42}$. Compared with term infants, relatively low baroreceptor reflex sensitivity values are found in the preterm infants, 10 and $4 \mathrm{~ms} / \mathrm{mmHg}$, respectively. A postnatal increase of the baroreceptor reflex sensitivity is observed in very preterm infants, however, with lower baroreceptor reflex sensitivity values found in very preterm infants reaching term compared to full-term born neonates ${ }^{31}$. For comparison, human adults have baroreceptor reflex sensitivity values of approximately $30 \mathrm{~ms} / \mathrm{mmHg}^{23}$. Thus, in contrast to the results obtained from fetal sheep, the baroreceptor sensitivity increases with gestational age in the human infant.

Spectral analysis has been used in fetuses ${ }^{52}$ and infants ${ }^{53,54}$ to estimate the relative contribution of the autonomic nervous system in maintaining cardiovascular homeostasis and determine the sympatho-vagal interactions regulating heart rate during resting conditions and after postural changes. Studies of fetal electrocardiogram tracings obtained on the maternal abdominal wall have shown that younger fetuses have a greater total energy of the power spectrum compared with more mature fetuses, consistent with the evolution of a stable and mature autonomic nervous system ${ }^{52}$. Maturational changes in the power spectra of heart rate variability have also been shown by comparing preterm to term infants ${ }^{53,54}$. There is a progressive decline in the low-to-high frequency power (LF/HF) ratio associated with both increasing postnatal and gestational age, indicating an increase in parasympathetic contribution to control of resting heart rate with maturation ${ }^{53.54}$. The high-frequency component in the power spectrum of heart rate significantly increased from preterm (31-36 wk) to term (37-38 wk) gestation, suggesting a significant increase in vagal tone at this age ${ }^{54}$. Power spectral analysis has also been used to characterize developmental changes in sympathovagal balance in response to arterial baroreceptor unloading in preterm infants beginning at 28-30 wk postconceptional age ${ }^{55}$. By longitudinally examining changes in heart rate power spectrum on a weekly basis, it was found that in infants at 28-30 wk the $\mathrm{LF} / \mathrm{HF}$ ratio did not change with head-up postural change, whereas with increasing 
postnatal age the low frequency component of the power spectrum increase with headup tilt. These findings suggest that neural regulation of cardiac function undergoes changes with maturation, becoming more functional with postnatal development. However these studies only evaluated heart rate variability without taking into account the concomitant fluctuations in blood pressure.

\subsection{Summary}

In this chapter the baroreceptor reflex is discussed as a negative-feedback control system. In human adults the arterial baroreceptor reflex mediated heart rate response is frequently studied by transiently increasing or decreasing arterial pressure. The arterial blood pressure - heart rate relationship resembles a sigmoid shape with a threshold (at low blood pressure) and saturation (at high blood pressure) level. Drug intervention will decrease or increase the input variable (arterial blood pressure) from the physiological point and enables to estimate (static open loop) gain from a dynamic closed loop system. The pharmacological method of altering the cardiovascular system, however, is not appropriate in neonates. Spectral analysis and transfer function analysis offer the opportunity to decompose spontaneously occurring fluctuations in blood pressure and $\mathrm{R}-\mathrm{R}$ interval series into a power spectrum and to estimate transfer gain and phase shift, respectively. With this method information about the baroreceptor reflex mediated heart rate response is limited to physiological gain and operating point.

Although investigators disagree about the way in which baroreceptor reflex sensitivity varies with pre- and postnatal maturation, they agree about the existence of a fetal functioning baroreceptor reflex in various animal models. The fetal sheep model shows a decreasing baroreceptor reflex sensitivity with age. By contrast, in the chicken embryo and in preterm human infants much lower baroreceptor reflex sensitivity values have been observed than at term age. Infants have in turn lower baroreceptor reflex sensitivity than human adults. Animal and human infant studies suggest that cardiovascular autonomic function undergoes changes with intra- and extra-uterine maturation with a progressive influence of the parasympathetic nervous system on heart rate variability postnatally. 


\section{References}

1. Sagawa K. Baroreflex control of systemic arterial pressure and vascular bed. In: Shepherd JT, Abboud FM (eds.) Handbook of physiology. Section 2, volume III, part 2. Bethesda, MD, American Physiological Society, 1983, pp 453-496

2. Arndt JO. Baroreceptors: morphology and mechanics of receptor zones and discharge properties of baroafferents. In: Gilmore JP, Zucker IH (eds) Reflex control of the circulation. CRC Press, Boca Raton, FL, 1991, pp 103-137

3. Abboud FM, Thames MD. Interaction of cardiovascular reflexes in circulatory control. In: Shepherd JT, Abboud FM (eds.) Handbook of physiology. Section 2, volume III, part 2. Bethesda, MD, American Physiological Society, 1983, pp 675-753

4. Zhang J, Mifflin SW. Subthreshold aortic nerve inputs to neurons in nucleus of the solitary tract. Am J Physiol 2000;278:R1595-RI604

5. Armour JA. Anatomy and function of the intrathoracic neurons regulating the mammalian heart. In: Gilmore JP, Zucker IH (eds) Reflex control of the circulation. CRC Press, Boca Raton, FL, 1991, pp $1-37$

6. Salata JJ, Zipes DP. Autonomic nervous system control of heart rate and atrioventricular nodal conduction. In: Gilmore JP, Zucker IH (eds) Reflex control of the circulation. CRC Press, Boca Raton, FL, 1991, pp 67-101

7. Levy MN. Martin PJ. Neuron control of the heart. In: Berne RM, Sperelakis N (eds.) Handbook of physiology, the cardiovascular system, American Physiological Society, Bethesda, 1979

8. Akselrod S, Gordon D, Madwed JB. Snidman NC, Shannon DC, Cohen RJ. Hemodynamic regulation: investigation by spectral analysis. Am J Physiol 1985:249:H867-H875

9. Milhorn HT. Application of control theory to physiological systems. Philadelphia, PA: Saunders, 1966

10. Grodins FS. Control theory and biological systems. New York: Columbia Univ Press, 1963

11. Guyton AC, Harris JW. Pressoreceptor-autonomic oscillation: a probable cause of vasomotor waves. Am J Physiol 1951:165:158-166

12. Ten Voorde B. Modeling the baroreflex: a systems analysis approach. Thesis 1992. Free University of Amsterdam. The Netherlands

13. Stauss HM. Baroreceptor reflex function. Am J Physiol 2002;283:R284-R286

14. Koch E. Die Blutdruckcharakteristik. Z Kreislaufforsch 1929;21:586-594

15. Wallin BG. Eckberg DL. Sympathetic transients caused by abrupt alterations of carotid baroreceptor activity in humans. Am J Physiol 1982;242:H185-90

16. Fritsch JM, Rea RF, Eckberg DL. Carotid baroreflex resetting during drug-induced arterial pressure changes in humans. Am J Physiol 1989;256:R549-R553

17. Akselrod $\mathrm{S}, \mathrm{Oz} \mathrm{O}$, Eliash $\mathrm{S}$. Neural and humoral factors in regulation of blood pressure in the investigation of essential hypertension. In: Di Rienzo M (ed.) Blood pressure and heart rate variability. IOS Press, Amsterdam. 1993, pp 192-206

18. Schmidt RM, Kumada M, Sagawa K. Cardiovascular responses to various pulsatile pressures in the carotid sinus. Am J Physiol 1972;223:1-7

19. Smyth HS. Sleight P. Pickering GW. Reflex regulation of arterial pressure during sleep in man: a quantitative method of assessing baroreflex sensitivity. Circ Res 1969;24:109-121

20. Eckberg DL, Cavanaugh MS, Mark AL, Abboud FM. A simplified neck suction device for activation of carotid baroreceptors. J Lab Med 1975;85:167-173

21. Pickering TG, Gribbin B, Strange Petersen E, Cunningham DJC, Sleight P. Effects of autonomic blockade on the baroreflex in man at rest and during exercise. Circ Res 1972:30:177-185 
22. Korner PI, Wets MJ, Shaw J, Uther JB. "Steady-state" properties of the baroreceptor-heart rate reflex in essential hypertension in man. Clin Exp Pharmacol Physiol 1974;1:65-76

23. Parlow J, Viale JP, Annat G, Hughson R, Quintin L. Spontaneous cardiac baroreflex in humans: comparison with drug-induced responses. Hypertension 1995;25:1058-1068

24. Murat I, Lapeyre G, Saint-Maurice C. Isoflurane attenuates baroreflex control of heart rate in human neonates. Anesthesia 1989;70:395-400

25. Scher AM, Ohm WW, Bumgarner K, Boynton R, Young AC. Sympathetic and parasympathetic control of heart rate in the dog, baboon and man. Federation Proc 1972;31:1219-1225

26. Eckberg DL. Nonlinearities of the human carotid baroreceptor-cardiac reflex. Circ Res 1980;47:208216

27. Leon DF, Shaver JA, Leonard. Reflex heart rate control in man. Am Heart J 1970;80:729-739

28. Laude D, Elghozi J, Girard A, Bellard E, Bouhaddi M, Castiglioni P, Cerutti C, Cividjian A, Di Rienzo M, Fortrat JO, Janssen B, Karemaker JM, Leftheriotis G, Parati G, Persson PB, Porta A, Quintin L, Regnard J, Rudiger H, Stauss HM. Comparison of various techniques used to estimate spontaneous baroreflex sensitivity (the EuroBaVar study). Am J Physiol 2004;286:R226-R231

29. Bertinieri G, Rienzo di M, Cavallazzi A, Ferrari AU, Pedotti A, Mancia G Evaluation of baroreceptor reflex by blood pressure monitoring in unanesthetized cats. Am J Physiol 1988;25:H377-H383

30. Drouin E, Gourmay V, Calamel J, Mouzard A, Rozé JC. Assessment of spontaneous baroreflex sensitivity in neonates. Arch Dis Childhood 1997:76:F108-F112

31. Gournay V, Drouin E, Rozé JC. Development of baroreflex control of heart rate in preterm and full term infants. Arch Dis Child Fetal Neonatal Ed 2002;86:F151-154

32. Penáz J. Mayer waves: history and methodology. Automedica 1978;2:135-141

33. de Boer RW, Karemaker JM, Strackee J. Hemodynamic fluctuations and baroreflex sensitivity in humans: a beat-to-beat model. Am J Physiol 1987;253:H680-H689

34. Robbe HWJ, Mulder LJM, Rüddel H, Langewitz WA, Veldman JBP, Mulder G. Assessment of baroreceptor reflex sensitivity by means of spectral analysis. Hypertension 1987; 10:538-543

35. Honzíková N, Fišer B, Honzík J. Noninvasive determination of baroreflex sensitivity in man by means of spectral analysis. Physiol Res 1992;41:31-37

36. Head GA, Lukoshkova EV, Burke SL, Malpas SC, Lambert EA, Janssen BJA. Comparing spectral and invasive estimates of baroreflex gain. IEEE Eng Med Biol 2001;20(2):43-52

37. Task Force of the European Society of Cardiology and the North American Society of pacing and electrophysiology. Heart rate variability: standards of measurement, physiological interpretation, and clinical use. Circulation 1996;93:1043-1065

38. Nuwayhid B, Brinkman CR, Su C, Bevan JA, Assali NS. Development of autonomic control of fetal circulation. Am J Physiol 1975:228:337-344

39. Woods JR, Dandavino A, Murayama K. Brinkman CR. Assali NS. Autonomic control of cardiovascular functions during neonatal development and in adult sheep. Circ Res 1977; 40:401-407

40. Assali NS, Brinkman CR, Woods JR, Dandavino A, Nuwayhid B. Development of neurohumoral control of fetal, neonatal, and adult cardiovascular functions. Am J Obstet Gynecol 1977;129:748759

41. Segar JL. Ontogeny of the arterial and cardiopulmonary baroreflex during fetal and postnatal life. Am J Physiol 1997;273:R457-R471

42. Altimiras J, Crossley II DA. Control of blood pressure mediated by baroreflex changes of heart rate in the chicken embryo (Gallus gallus). Am J Physiol 2000;278:R980-R986

43. Segar JL, Hajduczok G, Smith BA. Merrill DC, Robillard JE. Ontogeny of baroreflex control of renal sympathetic nerve activity and heart rate. Am J Physiol 1992;263:H1819-H1826

44. Blanco CE, Dawes GS. Hanson MA. McCooke HB. Carotid baroreceptors in fetal and newborn sheep. Pediatr Res 1988;24:342-346 
45. Gootman PM. Developmental aspects of reflex control of the circulation. In: Gilmore JP, Zucker IH (eds) Reflex control of the circulation. CRC Press, Boca Raton, FL, 1991, pp 965-1027

46. Waldman S, Krauss AN, Auld PAM. Baroreceptors in preterm infants: their relationship to maturity and disease. Develop Med Child Neurol 1979;21:714-722

47. Picton-Warlow CG, Mayer FE. Cardiovascular responses to postural changes in the neonate. Arch Dis Child 1970;45:354-359

48. Finley JP, Hamilton R, MacKenzie MG Heart rate response to tilting in newborns in quiet and active sleep. Biol Neonate 1984;45:1-1

49. Chen CM, Tsai TC, Lan MC. Effect of body tilting on physiological functions in healthy term neonates. Acta Paediatr 1995:84:474-477

50. Holden K, Morgan JS, Krauss AN, Auld PAM. Incomplete baroreceptor responses in newborn infants. Am J Perinatol 1985;2:21-34

51. Moss AJ, Emmanouilides GC, Monset-Couchard M. Marcano B. Vascular responses to postural changes in normal newborn infants. Pediatrics 1968;42:250-254

52. Karin J, Hirsch M, Akselrod S. An estimate of fetal autonomic state by spectral analysis of fetal heart rate fluctuations. Pediatr Res 1993;34:134-138

53. Chatow U, Davidson S, Reichman BL, Akselrod S. Development and maturation of the autonomic nervous system in premature and full-term infants using spectral analysis of heart rate fluctuations. Pediatr Res 1995:37:294-302

54. Clairambault J, Curzi-Dascalova L, Kaufmann F, Medigue C, Leffler C. Heart rate variability in normal sleeping full-term and preterm neonates. Early Hum Dev 1992;28:169-183

55. Mazursky JE, Birkett CL, Bedell KA, Ben-Haim SA, Segar JL. Development of baroreflex influences on heart rate variability in preterm infants. Early Hum Dev 1998:53:37-52 


\section{Chapter 3}

System network, acquisition and analysis of cardiovascular fluctuations 


\subsection{System network and environment}

The affordability and increased power of the current generation of computers allow the development of applications with sophisticated hardware and many software development tools. In order to obtain a more precise knowledge of the cardiovascular system in neonates the Department of Clinical Physics and the neonatal intensive care developed 10 years ago a central data acquisition system with a local area network, that allowed continuous registration of heart rate (ECG), intra-arterial blood pressure and respiration (thoracic impedance) at each intensive care bed ${ }^{1}$. The global architecture of the data acquisition system is shown in Figure 1.

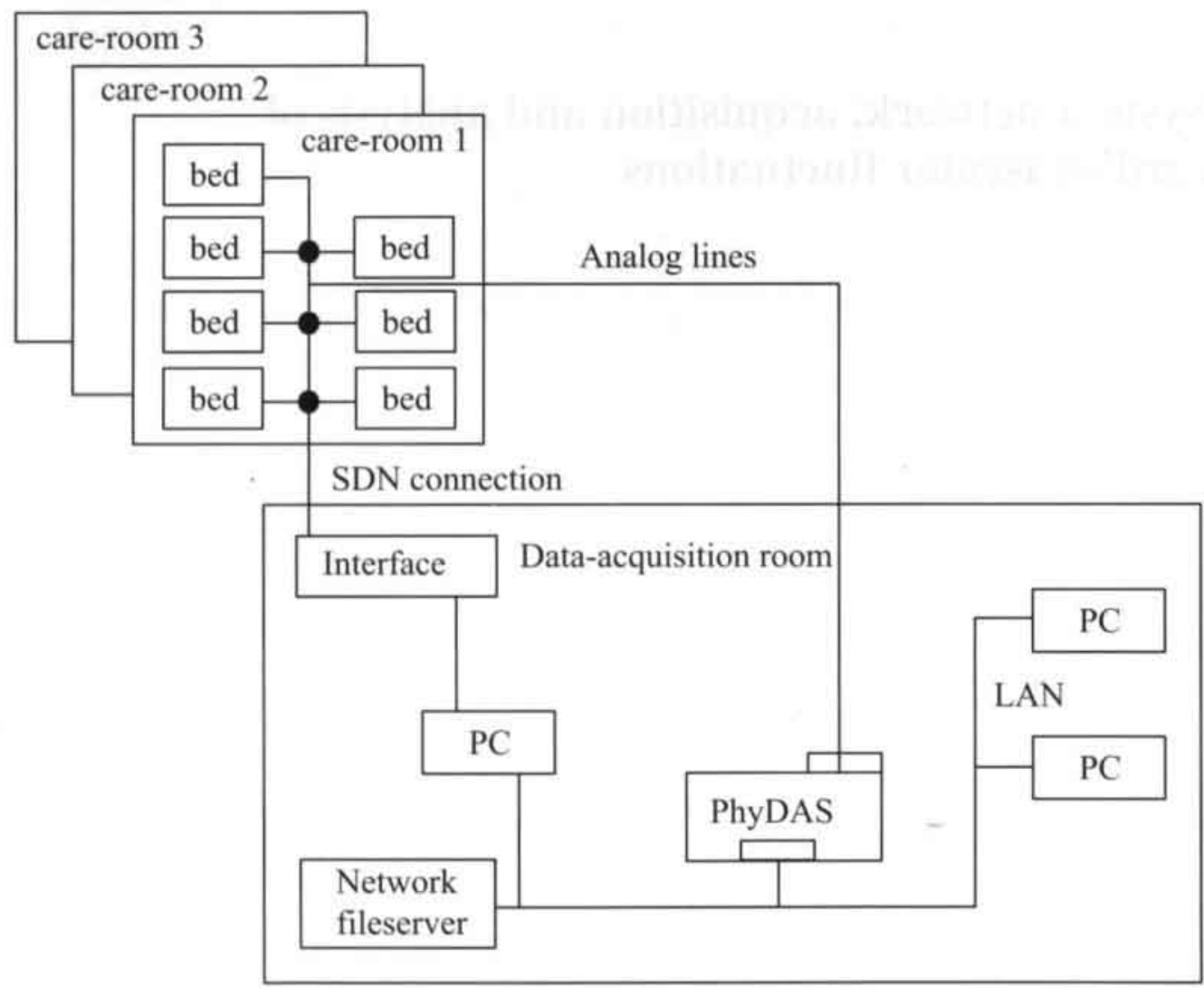

Figure 1. After: de Jong 2000. The diagram shows the global architecture of the physics data acquisition system (PHYDAS) and the environment of the neonatal intensive care unit. Personal computer (PC), local area network (LAN), Serial Distribution Network (SDN). 
In the period 2000-2003, each intensive care bed was equipped with a neonatal Hewlett Packard monitor, type Merlin (Hewlett Packard, Waltham, MA, US). The monitor system consists of a module rack, click-in modules, computer with screen, connection with an HP Serial Distribution Network (SDN) and an analog output interface. The SDN served for storage of averaged and filtered parameter data. The analog raw and unfiltered waveform signals (ECG, arterial blood pressure and thoracic impedance) were transferred to a central data acquisition system. The analog output module (D/A conversion at $500 \mathrm{~Hz}$ ) supplies a maximum of 8 user-defined signals. The digitized signals were transferred to the local area network and stored on the server for off-line analysis. Several software packages, written in Labview, were developed to archive, visualize and analyze the signals.

The data acquisition system has been developed at the faculty of Physics of the Eindhoven University of Technology, Eindhoven, The Netherlands in collaboration with the department of Clinical Physics and the Neonatal Intensive Care Unit of the Máxima Medical Center in Veldhoven.

\subsection{Data acquisition and subjects}

A bipolar chest lead of the surface ECG and the transthoracic electric impedance waveforms were recorded by Hewlett Packard neonatal monitor type Merlin. Arterial blood pressure was measured invasively by a fluid-filled catheter manometer system connected with a peripheral arterial catheter or a 4 French catheter placed earlier in the aortic position for routine monitoring of vital functions and intensive care management. A $0.5 \mathrm{ml} / \mathrm{h}$ infusion of heparinized physiological saline solution was continuously flushed through the catheter. The ECG and arterial blood pressure signals were sampled at a rate of $512 \mathrm{~Hz}$ and $128 \mathrm{~Hz}$, respectively. The feasibility of measuring blood pressure variability by a noninvasive beat-to-beat finger arterial blood pressure device (Finapres), with the application of the finger cuff around the infant's wrist, was assessed in preterm infants and is discussed in chapter 5 .

Several physiological and pathological conditions influence neonatal cardiovascular variability. Among the physiological conditions are gestational age, behavioral state and body position. Heart rate decreases and heart rate variability increases with gestational age and early postnatal age. In addition, a gradually increase in the low-to-high spectral power of heart rate variability is observed with gestational age ${ }^{2.3}$. The high frequent component in the power spectrum of heart rate significantly increases from preterm (31-36 wk) to term (37-38 wk) gestation ${ }^{4}$. Total variability in heart rate is higher in 
active sleep state than in quiet sleep state 5 . In prone position, infants show higher heart rate and lower total variability compared to the supine position ${ }^{6}$. Among the pathological conditions are compromised lung function, brain insult, patent ductus arteriosus, growth retardation and medication. Total variability is attenuated in subjects with an active respiratory distress syndrome, while mechanical ventilation influences the high frequent spectral power due to changes in ventilation frequency ${ }^{7.8}$. Asphyxia and intra-ventricular hemorrhage are associated with reduction in heart rate variability 9,10 . Compared with controls, subjects with a patent ductus arteriousus show higher heart rate and lower heart rate variability ${ }^{8}$. The higher heart rate may induce an increase in transductal shunting which is known to be associated with an increase in high frequent blood pressure variability in preterm infants with respiratory distress syndrome"l. Small-for gestational age infants have higher heart rate and lower variability than controls ${ }^{12}$.

The implications of these conditions influencing variability lead to standardize the methods of measurements and to formulate the subject group as follows:

1. all measurements (heart rate, arterial blood pressure, respiration, oxygen saturation and observation of sleep state) in the prone position, not earlier than 24-hours of delivery; data acquisition preferentially between 12:00 and 24:00 for 1-2 hours;

2. inclusion: appropriate-for-gestational age infants, clinically judged as cardiorespiratory stable or resolving respiratory distress syndrome, spontaneous breathing room air;

3. exclusion: congenital diseases, intraventricular hemorrhage or periventricular leucomalacia grade II or higher ${ }^{13,14}$; Apgar score below 6 at 5 minute; umbilical arterial $\mathrm{pH}$ below 7.0; drugs other than antibiotics or caffeine; patent ductus arteriosus or fluid expanders at or around the time of measurements.

In two studies (chapter $6 \& 8$ ) recordings in stable infants were made in the prone position for 1-2 hours and data segments were selected subsequently during periods of regular breathing and spontaneous sleep with closed eyes and without gross body movements (quiet sleep state) ${ }^{15}$. Because preterm infants have an immature and irregular respiratory drive, 192-s segments were chosen as a compromise between the demands of sufficient duration and signal stability. Variability analysis was performed on these 192-s-long stationary segments. In the atropine study (chapter 7) longer periods were analyzed without taking the sleep state into account. The character of the "acute" intervention precluded selecting periods of quiet sleep state. 


\subsection{The rationale for frequency domain analysis}

In the human being, the sinoatrial node located at the posterior wall of the right atrium, initiates each beat of the heart. The spontaneous membrane depolarizations of the sinoatrial myocytes are modulated by various factors that add variability to the heart rate signal at different frequencies. According to the Task force of the European Society of Cardiology and The North American Society of Pacing and electrophysiology these frequencies are classified into 1) ultra-low frequencies $(<0.003 \mathrm{~Hz})$ that include circadian rhythm, 2) very low frequencies $(0.003-0.04 \mathrm{~Hz})$ that are supposed to be affected by temperature regulation and humoral systems, 3 ) low frequencies (0.04-0.15 $\mathrm{Hz}$ ) that are attributed to baroreceptor reflex activity and sensitive to changes in cardiac sympathetic and parasympathetic nerve activity, and 4) high frequencies $(0.15-0.4 \mathrm{~Hz})$ that are synchronized to the respiratory rhythm and are primarily modulated by cardiac parasympathetic innervation. The ratio of low (reflecting sympathetic and parasympathetic activity) to high (reflecting parasympathetic activity) spectral power of heart rate variability is often used as a tool to determine "autonomic balance". Thus, sympathovagal balance is simply the ratio of low-to-high frequent spectral power (dimensionless unit) ${ }^{16}$. A high ratio seems to be an unfavorable prognostic factor for cardiovascular diseases. The prognostic importance of the concepts of heart rate variability following myocardial infarction has clearly demonstrated ${ }^{17}$. However, established investigators have diametrically opposing views regarding the concept of sympathovagal balance. Malliani noted that the reciprocal relationship between low and high frequency assesses the sympathovagal balance ${ }^{18}$. Eckberg stated that "calculations of sympathovagal balance (ratio of low-to-high frequent spectral power) may obscure rather than illuminate human physiology and pathophysiology"19. Others suggest using the intrinsic R-R interval following autonomic blockade or (more practical) the resting $\mathrm{R}-\mathrm{R}$ interval as a measure of sympathovagal balance ${ }^{20}$.

Thus, heart rate variability may be viewed as a set of fluctuations at different frequencies which are attributed to physiological mechanisms. Fourier transform is an analysis technique which enables to decompose the various frequency components into a power spectrum. In this thesis I focus on the low frequent fluctuations (approximately $0.1 \mathrm{~Hz}$ ) which are supposed to be associated with baroreceptor reflex activity and high frequent fluctuations related to respiratory activity. In the following section I discuss in more detail the frequencies of interest in neonates. 


\subsection{Frequency ranges of interest}

The Task Force has defined the frequency bands for the application in human adults ${ }^{16}$. The regular distribution of a spectrum of heart rate variability of a human adult includes a peak at the frequency of Mayer's wave (around $0.1 \mathrm{~Hz}$ ) and a peak at the breathing frequency (respiratory sinus arrhythmia, at approximately $0.25 \mathrm{~Hz}$ ). These recommendations may not be appropriate for neonates due to a shift of the spectrum of the high frequencies 21,22 . Neonates have considerable higher respiratory rates than adults and may vary between 30 and 90 breaths per minute, similar to 0.5 and $1.5 \mathrm{~Hz}$, respectively. Likewise, the neonatal heart rate may vary between 100 and 200 beats per minute, similar to 1.7 and $3.3 \mathrm{~Hz}$, respectively. As the neonatal heart rate and respiratory rates differ from that of the adult, neonatal studies have used different spectral divisions. The cut-off points between the low frequent and high frequent band used in several neonatal studies are summarized in Table 1. Unlike the consensus for spectral analysis in human adult, there is no agreement in spectral band definitions for neonatal studies. There are several reasons for this. First, the respiratory rate in preterm infants fluctuates considerably. Preterm infants have an immature respiratory drive with periodic breathing or apneas. Therefore the high frequent spectral peak in neonates may be dispersed. Second, as a consequence the signal is only stable for a relative short time which limits an appropriate signal analysis. Third, the appropriate upper spectral border (Nyquist critical frequency) presents an additional problem as will be discussed in chapter 3.5 .

Based on the available literature the frequency ranges of interest are defined as follows. The whole frequency band of interest was the range between 0.04 and $1.5 \mathrm{~Hz}$. The very low frequency band $(<0.04 \mathrm{~Hz})$ was discarded to avoid possible contribution of slow trend artifacts. Fast Fourier transform of 64-s segment length has limited accuracy of power estimation in this frequency range using records of relatively short duration. The spectral density is considered reliable if at least three cycles of periodicity are included in the recording 23 . Thus, recordings of 3-min length have a low frequency cut-off point of $0.017 \mathrm{~Hz}$ ( 1 cycle per minute). Averaging five spectra within a 3-min sample reduces the relative error of mean spectral power to $23 \%$ per frequency bin. Using $64-\mathrm{s}$ segments (resulting in a spectral resolution of $0.016 \mathrm{~Hz}$ ) and 5 half-overlapping segments power estimation above $0.04 \mathrm{~Hz}$ was considered to be acceptable. Thus, the low frequency band was defined as the frequency range between 0.04 and $0.15 \mathrm{~Hz}$. Because the high frequency band primarily contains the reflection of respiratory activity, this band was individualized for each subject depending on his or her respiratory drive. The bandwidth between the $10^{\text {th }}$ and $90^{\text {th }}$ centile of the breath by 
breath frequency was used to identify each subject's individual high frequency band. The upper spectral limit of the high frequency band (the $90^{\text {th }}$ centile of the respiratory frequency) was less than half of the mean HR thereby meeting the requirements of the Nyquist critical frequency (see chapter 3.5).

Table 1: Low frequency (LF) and high frequency (HF) band

\begin{tabular}{|c|c|c|c|}
\hline reference & population & LF $(\mathbf{H z})$ & $\mathbf{H F}(\mathbf{H z})$ \\
\hline Giddens $1985^{24}$ & full-term & $0.04-0.2$ & $0.2-?$ \\
\hline Dykes $1986^{25}$ & full-term & $0.02-0.2$ & $0.2-?$ \\
\hline Aarimaa $1988^{7}$ & preterm; full-term & $0.02-0.2$ & $0.2-1$ \\
\hline Baldzer $1989^{26}$ & full-term & $0.04-0.16$ & RSA $\$$ \\
\hline Ravenswaaij 199427 & preterm & $0.04-0.2$ & RSAs \\
\hline Eiselt $1993^{28}$ & preterm; full-term & $0.03-0.1$ & $0.4-1$ \\
\hline Spassov $1994^{12}$ & full-term & $0.03-0.1$ & $0.4-1$ \\
\hline Chatow $1995^{2}$ & preterm; full-term & $0.02-0.2$ & $0.2-2$ \\
\hline Mazursky $1998^{3}$ & preterm & $0.02-0.15$ & $0.15-1.5$ \\
\hline Sahni 19996 & preterm; full-term & $0.05-0.2$ & $0.5-2.0$ \\
\hline Veerappan $2000^{29}$ & preterm & $0.03-0.40$ & $0.4-1.0$ \\
\hline Franco $2000^{30}$ & full-term & $0.04-0.15$ & $\mathrm{p}-25$ to $\mathrm{p}-75^{\#}$ \\
\hline
\end{tabular}

$\$$, the respiratory sinus arithmia (RSA) was defined as a band within $\pm 0.2 \mathrm{~Hz}$ of the respiratory frequency obtained from peak amplitude in the respiratory power spectrum; \# individualized respiratory bandwidth, defined between $\mathrm{p}-25$ and $\mathrm{p}-75$ of the respiratory power spectrum.

\subsection{Fast Fourier transform}

The Fourier transform is a mathematical method that changes (transforms) data from a quantity varying over time to (possibly complex) amplitude varying over a range of frequencies. Another way to view Fourier transform is that it fits harmonic equations to time-varying data, yielding the frequency components of the original signal. The original signal is often called $x(t)$ and the resultant function of frequency $X(f)$. If the units of $\mathrm{t}$ are seconds, the units of $\mathrm{f}$ will be $\mathrm{s}^{-1}$ or Herz $(\mathrm{Hz})$. Since $x(t)$ is a function of time, it is in the time domain. $X(f)$ is a function of frequency, and thus is in the frequency domain. Under appropriate conditions the Fourier transform is reversible: $x(t) \rightarrow X(f)$ and $X(f) \rightarrow x(t)$. Thus the variance of the signal (root-mean-square for the mean) is similar to the total spectral power. Likewise, spectral power analysis provides the basic information how the variance (or power) is distributes as a function of 
frequency. While continuous functions are transformed using continuous transforms, discrete sampled data requires the discrete Fourier transform. The fast Fourier transform is merely a Fourier transform that greatly speeds computation ${ }^{31}$.

The fast Fourier transform was used to compute the auto- and cross spectral density functions for each segment. With respect to the mathematical details of the fast Fourier transform I refer to appendix A.

Four common distortions may arise in using the fast Fourier transform: 1) aliasing, 2) spectral leakage, 3) errors due to non-equidistant sampling and 4) errors due to a lack of stationarity ${ }^{32}$.

1. Aliasing occurs in Old West movies, where the wagon wheel spokes appear to move backwards as the wagon accelerates. The sampling theorem states that an accurate an accurate determination of spectral components is possibly only at frequencies no greater than the Nyquist critical frequency, determined as one-half of the sampling rate. Therefore if fluctuations in heart rate are under investigation, the high frequent cut-off point should be half the lowest normal mean heart rate. Signals at frequencies above the Nyquist critical frequency will be erroneously translated to below the Nyquist limit in $X(f)$. Assuming the heart rate to be 150 beats per minute (equal to $2.5 \mathrm{~Hz}$ ), the Nyquist criterion requires an upper limit of the respiratory frequency of 75 breaths per minute (equal to $1.25 \mathrm{~Hz}$ ).

2. Oscillations at frequencies that produces exactly 1,2,3, etc., cycles over the record duration will produce narrow peaks at the corresponding frequencies. However, any other oscillation frequency will produce a broad peak, plus additional effects throughout the measured frequencies. Tapering the end of the time-domain sample removes discrepancy between start and end, minimizing spectral leakage. Tapering is achieved by a time-domain window (e.g. a triangle shaped Parzen window).

3. The time-domain data for transformation are assumed to be sampled at a constant interval. However, the raw heart rate can only be computed after a beat occurs. Because beats occur at varying rates, new heart rates are available at irregular intervals. Interpolation and resampling of the time series produces equidistant sampling of the signal.

4. Fourier transform methods assume stationarity: the data segment has the same spectral characteristics throughout. However, this is difficult to assure in physiological studies. The compromise is between the reduced resolution of short collection periods and the spectral distortions from non-stationarity over longer periods. In chapter 4 a comparison between two applications of analysis procedures is discussed. 


\subsection{Data analysis}

I refer to appendix A for the (mathematical) details of the variability analysis. Briefly, $\mathrm{R}$ waves were detected from the ECG and an unevenly spaced R-R interval sequence was created. This sequence was resampled using a boxcar window at $4 \mathrm{~Hz}$ in order to obtain an equidistantly spaced time series ${ }^{32}$. The quality of the data was visually inspected for ectopic beats, missing data and baseline drift ${ }^{16}$. $\mathrm{R}-\mathrm{R}$ interval artifacts were removed and linearly interpolated. The mean $R-R$ interval was calculated and subtracted from all data points to remove the direct current component. Systolic blood pressure was identified from peak detection of the blood pressure signal, resulting in an unevenly spaced "systogram". The "systogram" was converted into an equidistantly spaced time series using the same resampling method as used for the R-R interval time series. As stated before (chapter 3.2) variability analysis was performed on 192-s-long stationary segments. Each 192-s-long segment of preprocessed evenly spaced R-R interval and systolic blood pressure series was subdivided in 5 half-overlapping 64-s (256 points) segments. To reduce the effects of spectral leakage each segment was multiplied with a triangular Parzen window ${ }^{33}$. Fast Fourier transform was used to compute the auto- and cross spectral density functions for each 256 points segment. The spectral resolution was $0.016 \mathrm{~Hz}$. A filter correction removed unwanted side effects of the earlier resampling and Parzen windowing ${ }^{32}$. A mean power spectrum was derived from the 5 autospectral density functions. Powers in the ranges of interest were calculated from the obtained mean autospectral density functions. Using 5 halfoverlapping 64-s segments and Parzen windowing resulted in a relative error in mean spectral power of $23 \%$ per frequency bin ${ }^{34}$. The values for power are presented in units of $\mathrm{ms}^{2}$ (for $\mathrm{R}-\mathrm{R}$ interval series) and $\mathrm{mmHg}^{2}$ (for systolic blood pressure series) or expressed as normalized units [e.g. low frequent power/total power x $100 \%$ ].

Figure 2 shows the time series of the R-R and systolic blood pressure values in a preterm infant at a postconceptional age of 30 weeks. Note that in the systolic blood pressure series two different fluctuations can be seen: relatively slow fluctuations of greater amplitude (approximately 17 fluctuations in the 192-s sample, equal to approximately $0.1 \mathrm{~Hz}$, amplitude $2-4 \mathrm{mmHg}$ ) and relatively fast fluctuations of small amplitude (approximately 20 fluctuations in $25 \mathrm{~s}$, equal to approximately $0.8 \mathrm{~Hz}$, amplitude $1 \mathrm{mmHg}$ ). Figure 3 illustrates the spectral density curves for R-R interval and systolic blood pressure series of the same infant as in Figure 2, with the boundaries marked for the low $(0.04-0.15 \mathrm{~Hz})$ and high frequency (p-10 and p-90 centile: $0.70-0.95$ $\mathrm{Hz}$ ) band, respectively. The spectral density curves confirm the visual interpretation of the time series of R-R interval and systolic blood pressure values as shown in Figure 2. In the low frequency band a clear spectral peak is observed at $0.09 \mathrm{~Hz}$. The low frequent 
peak at $0.09 \mathrm{~Hz}$ is assumed to be attributed to oscillation of the baroreceptor reflex. The high frequent peak at approximately $0.8 \mathrm{~Hz}$ corresponds with the mean respiratory rate of the subject (respiratory sinus arrhythmia).

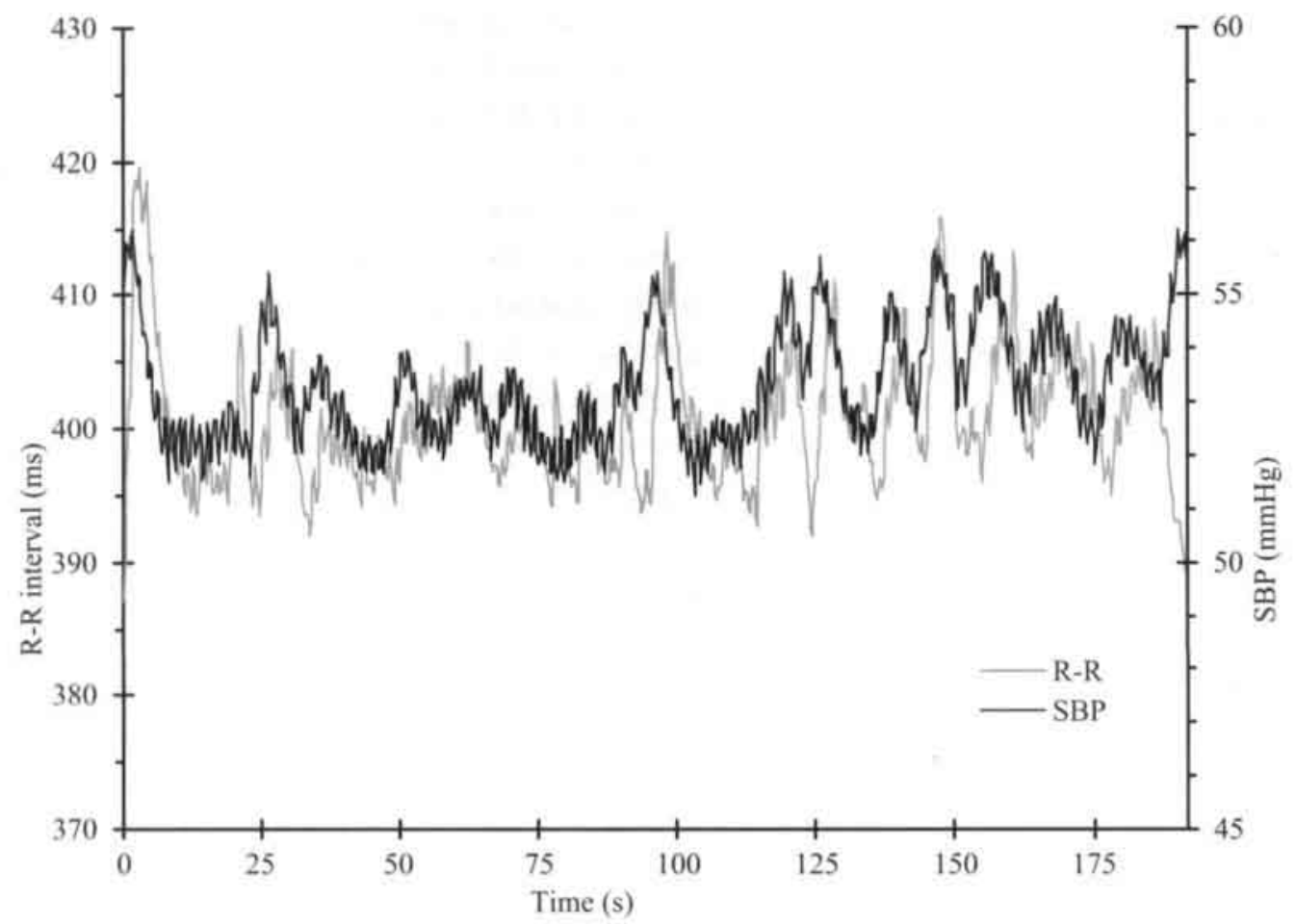

Figure 2. The figure shows the time series of the R-R and systolic blood pressure values for a 192-s period ( $x$ axis) in a preterm infant during quiet sleep state at a postconceptional age of 30 weeks. The left and right $y$ axes express the $\mathrm{R}-\mathrm{R}$ interval in $\mathrm{ms}$ and systolic blood pressure (SBP) in $\mathrm{mmHg}$. respectively.

Note that in the systolic blood pressure series two different fluctuations can be seen: relatively fast fluctuations of small amplitude (approximately 20 fluctuations in $25 \mathrm{~s}$, equal to $0.8 \mathrm{~Hz}$, amplitude $1 \mathrm{mmHg}$ ) and relatively slow fluctuations of greater amplitude (approximately 17 fluctuations in $192 \mathrm{~s}$. equal to approximately $0.1 \mathrm{~Hz}$, amplitude $2-4 \mathrm{mmHg}$ ). 

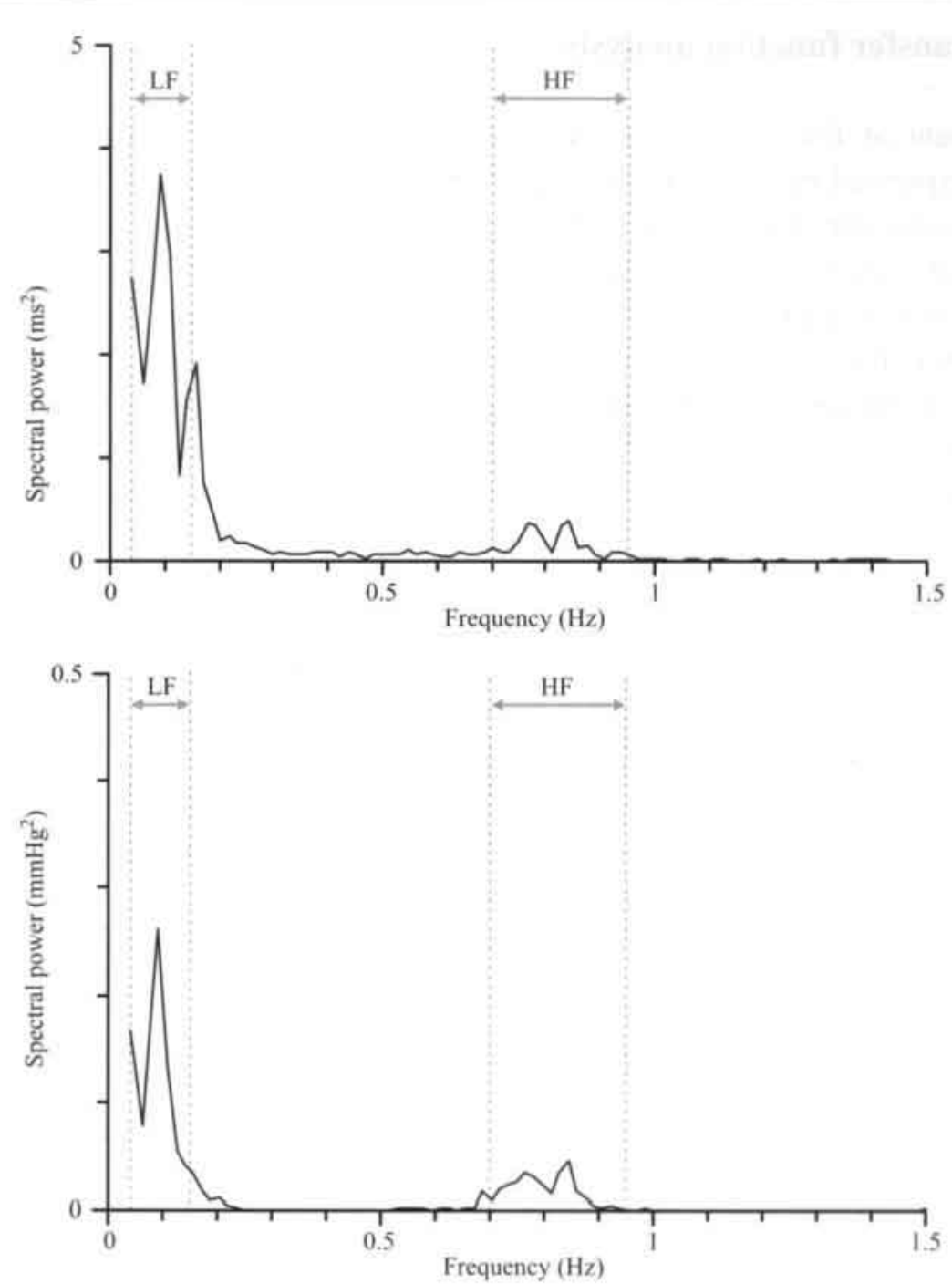

Figure 3. This picture shows the spectral power curves of R-R interval series (upper panel) and spectral power curve of systolic blood pressure (SBP) series (lower panel) of the same infant as in Figure 2. The spectral power ( $\mathrm{R}-\mathrm{R}$ interval series, $\mathrm{ms}^{2}$; SBP series $\mathrm{mmHg}^{2}$ ) is distributed as a function of frequency $(\mathrm{Hz})$. The low frequency (LF) band was defined between 0.04 and $0.15 \mathrm{~Hz}$ and is marked between vertical lines. The individual high frequency (HF) band was defined between the $10^{\text {th }}$ and $90^{\text {th }}$ centile of the individual respiratory frequency $(0.70$ and $0.95 \mathrm{~Hz}$, respectively) and is marked between vertical lines.

The spectral density curves confirm the visual interpretation of the time series of R-R interval and systolic blood pressure values as shown in Figure 2. In the LF band a clear spectral peak is observed at $0.09 \mathrm{~Hz}$. The LF peak of $0.09 \mathrm{~Hz}$ is assumed to be attributed to oscillation of the baroreceptor reflex. The HF peak at approximately $0.8 \mathrm{~Hz}$ corresponds with the mean respiratory rate of the subject (respiratory sinus arrhythmia). 


\subsection{Transfer function analysis}

The amount of linear coupling between two variables in the frequency domain is usually expressed by the coherence function ${ }^{35}$. The coherence of time series $x(t)$ and $y(t)$ measures the variance of $y$ linearly predictable from $x$ at each frequency $f$. Coherence serves as the frequency domain analog of $r^{2}$, the coefficient of determination. Like $r^{2}$, its value lies between 0 and 1 , with values near 1 indicating a strong linear relation between the two series. Thus, the coherence level indicates the strength of the linear association and provides an estimate of variability in phase between two variables (e.g. blood pressure and R-R interval series). After the work of de Boer et al, it has become common to claim a significant linear relation between two cardiovascular time series when coherence values exceed 0.5 . The level of 0.5 indicates a relation between two signals based on $50 \%$ shared variance.

The transfer function gain and phase between blood pressure and R-R interval series were estimated from the auto- and cross spectral density functions using a fast Fourier technique. Transfer gain and phase were assessed in the low $(0.04-0.15 \mathrm{~Hz})$ and high frequency ( $\mathrm{p}-10$ and $\mathrm{p}-90$ centile) band at the frequency of highest coherence. The gain $(\mathrm{ms} / \mathrm{mmHg}$ ) reflects the degree to which the input signal (blood pressure) amplitude becomes manifest in the output signal ( $R-R$ interval) amplitude at a discrete frequency. With respect to the mathematical details of the squared coherence and transfer function analysis I refer to appendix A.

The low frequent transfer gain was used to estimate baroreceptor sensitivity, as discussed in chapter 2.4. The phase (degrees or s) of the transfer function indicates the temporal relationship between the signals in the frequency domain ${ }^{36}$. However, in case of an intact (baroreceptor) closed loop system, judgment considering causality and interpretation of the phase spectrum is hampered, because it is not clear whether one signal leads another by $360^{\circ}+n$ degree or is behind by $360^{\circ}-n$ degree. From Figure 2 and 3 one may observe that the baroreceptor reflex oscillation frequency is $0.09 \mathrm{~Hz}$, equal to a wave length of $11 \mathrm{~s}$. Figure 4 illustrates the transfer function parameters (coherence, gain and phase) between systolic blood pressure and R-R interval signals of the same preterm subject as in Figure $2 \& 3$. Note that high coherence values (notably above 0.5 ) are calculated for the low and high frequency band. The frequency values with the highest coherence in the low and high frequency band were chosen to compute transfer gain and phase. The corresponding low and high frequent transfer gain and phase are indicated by dots in the Figure 4 . In this subject, baroreceptor sensitivity (gain) was $3.5 \mathrm{~ms} / \mathrm{mmHg}$. The calculated phase difference between the low frequent 

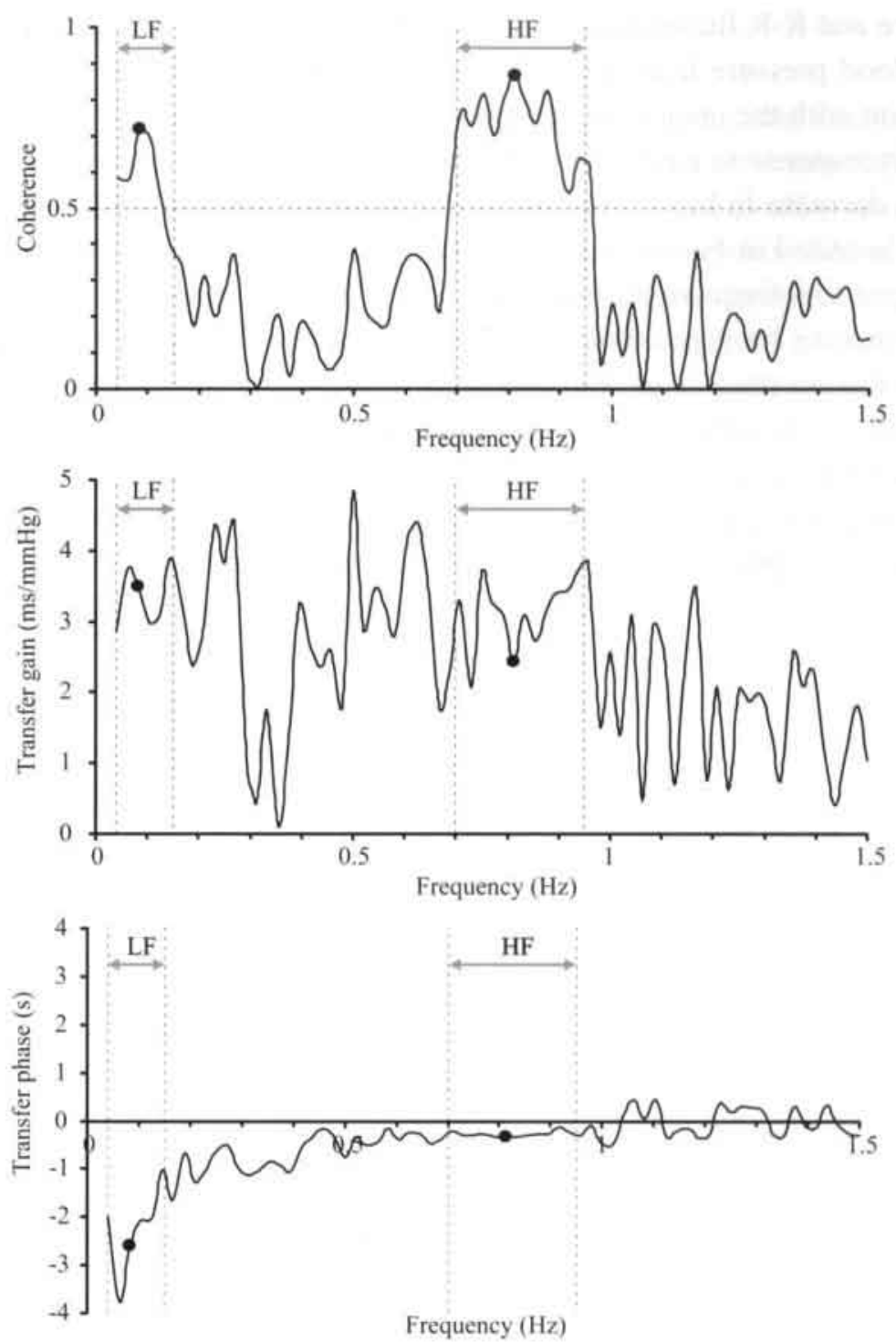

Figure 4. This figure shows the coherence function for the linear relationship between systolic blood pressure (SBP) and R-R interval series (top) and corresponding transfer gain (middle) and transfer phase (bottom) as a function of frequency. The low frequency (LF) band was defined between 0.04 and $0.15 \mathrm{~Hz}$ and is marked between vertical lines. The individual high frequency (HF) band was defined between the $10^{\text {th }}$ and $90^{\text {th }}$ centile of the individual respiratory frequency $(0.70$ and $0.95 \mathrm{~Hz}$, respectively) and is marked between vertical lines. Note the high coherence values in both LF and HF bands. The horizontal line (top) indicates the coherence value of 0.5 ; above this value a reliable estimate was considered for the transfer function parameters gain and phase.

The frequency values with highest coherence in LF and HF were chosen to compute gain and phase (indicated as dots). At L.F, transfer gain was calculated $3.5 \mathrm{~ms} / \mathrm{mmHg}$ and transfer phase $2.5 \mathrm{~s}$. 
blood pressure and R-R fluctuations was $2.5 \mathrm{~s}$. I interpreted this that at low frequency, changes in blood pressure lead the change in R-R interval for the following reasons. First, consistent with the open loop approach of the baroreceptor reflex is $\mathrm{R}-\mathrm{R}$ interval lengthening in response to an increase in blood pressure, or R-R interval shortening in response to a decrease in blood pressure ${ }^{37}$. Second, the temporal relationship between the signals, illustrated in Figure 5, showing the last $60 \mathrm{~s}$ of the time series of Figure 1, strongly suggest low frequent fluctuations in systolic blood pressure rise precede R-R intervals lengthening for approximately $2.5 \mathrm{~s}$. It is highly unlikely that at low frequency, $R-R$ interval changes precede arterial pressure changes with a latency of $11-2.5=8.5 \mathrm{~s}$. In case of heart rate frequencies of $2.5 \mathrm{~Hz}$ and respiratory frequencies of approximately $0.8 \mathrm{~Hz}$, taken into account the closed loop properties, interpretation of the phase shift in the high frequent range is not possible. It is not clear whether high frequent fluctuations in blood pressure lead or lag fluctuations in R-R intervals (Figure 5).

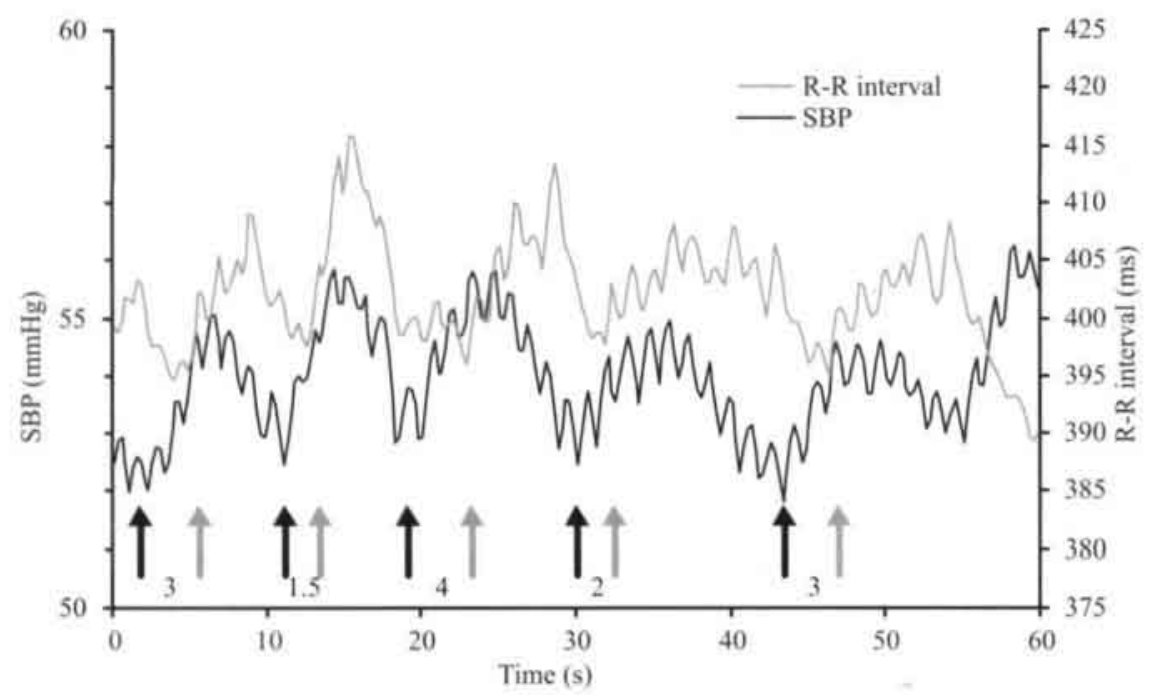

Figure 5. This figure shows a 60-s trace of R-R interval and systolic blood pressure values of the 3-min segment, as presented in Figure 1. It shows the temporal relationship between systolic blood pressure (SBP) and R-R interval fluctuations. SBP (left $y$ axis) and R-R interval (right $y$ axis) values are shown as a function of time. High frequent fluctuations with small amplitude variation, related to respiratory activity, can be observed. In addition, approximately six low frequent fluctuations with a variation of $3 \mathrm{mmHg}$ per cycle in this 60-s trace can be observed, corresponding to an oscillation frequency of approximately $0.1 \mathrm{~Hz}$. Each rise in SBP (indicated by the first arrow) is followed by an increase in R-R interval (indicated by the second arrow), with a time delay varying from 1.5 to $4 \mathrm{~s}$ (periods between arrows). The averaged time delay from this $60-\mathrm{s}$ trace $(2.7 \mathrm{~s})$ is close to the calculated transfer phase. 


\subsection{Summary}

The cardiovascular system contains negative-feedback (gain) and time-delay (phase) mechanisms resulting in oscillations in heart rate and blood pressure, which are apparent when these signals are measured over time. Specifically, spectral analysis allows quantification of heart rate and blood pressure variability. Alterations in heart rate and blood pressure variability as evidenced from the spectral characteristics of these physiological signals allow the clinicians to have indicators of neural autonomic control. Interestingly, parasympathetic-mediated changes in heart rate occur much faster than sympathetic-mediated effects on heart rate.

In this thesis I focus on the low frequent fluctuations $(0.04-0.15 \mathrm{~Hz})$ which are supposed to be associated with baroreceptor reflex activity and related to sympathetic and parasympathetic activity. High frequent fluctuations are associated with respiratory activity (respiratory sinus arrhythmia) and related to parasympathetic activity.

The low-to-high frequent spectral power of heart rate variability and resting heart rate are used in human adults as tools to determine the "sympathovagal balance".

Transfer function gain at frequencies related to baroreceptor oscillations $(0.04-0.15 \mathrm{~Hz})$ reflects the degree to which the input signal (arterial blood pressure) amplitude becomes manifest in the output signal ( $R-R$ interval) amplitude and may be used as an estimate for baroreceptor sensitivity. Transfer function phase indicates the temporal relationship between the signals in the frequency domain. In the following studies (chapter 6-8) the transfer function parameters are the key estimates of baroreceptor reflex function. 


\section{References}

1. de Jong W. Blood pressure variability in neonates: with a special focus on signal acquisition and signal processing. PhD Thesis 2000, Eindhoven University of Technology, Eindhoven, The Netherlands

2. Chatow U, Davidson S, Reichman BL, Akselrod S. Development and maturation of the autonomic nervous system in premature and full-term infants using spectral analysis of heart rate fluctuations. Pediatr Res 1995;37:294-302

3. Mazursky JE, Birket CL, Bedell KA, Ben-Haim SA, Segar JL. Development of baroreflex influences on heart rate variability in preterm infants. Early Hum Dev 1998:53:37-52

4. Clairambault J, Curzi-Dascalova L, Kaufmann F. Medigue C. Leffler C. Heart rate variability in normal sleeping full-term and preterm neonates. Early Hum Dev 1992;28:169-183

5. Curzi-Dascalova L. Physiological correlates of sleep development in premature and full-term neonates. Neurophysiol Clin 1992;22:151-166

6. Sahni R, Schulze KF, Kashyap S, Ohira-Kist K, Fifer WP, Myers MM. Postural differences in cardiac dynamics during quiet and active sleep in low birth infants. Acta Paediatr 1999:88:1396-1401

7. Aärimaa T, Oja R, Antila K, Välimäki I. Interaction of heart rate and respiration in newborn babies. Pediatr Res 1988;24:745-750

8. van Ravenswaaij-Arts CMA, Hopman JCW, Kolleé LAW, van Amen JPL, Stoelinga GBA, van Geijn HP. The influence of respiratory distress syndrome on heart rate variability in very preterm infants. Early Hum Dev 1991;27:207-221

9. Divon MY, Winkler H, Yeh SY, Platt LD, Langer O, Merkatz IR. Diminished respiratory sinus arrhythmia in asphyxiated term infants. Am J Obstet Gynecol 1986;155:1263-1266

10. Prietsch V, Knoepke U, Obladen M. Continuous monitoring of heart rate variability in preterm infants. Early Hum Dev 1994:37:117-131

11. Beuchée A, Pladys P, Senhadji L, Bétrémieux P, Carré F. Beat-to-beat pressure variability and patent ductus arteriousus in ventilated, premature infants. Eur J Physiol 2003:446:154-160

12. Spassov L, Curzi-Dascalova L, Clairambault J, Kauffmann F, Eiselt M, Medigue C, Peirano P. Heart rate and heart rate variability during sleep in small-for-gestational age newborns. Pediatr Research 1994:35:500-505

13. Papile LA, Burstein J, Burstein R. Koffler H. Incidence and evolution of subependymal and intraventricular haemorrhage: a study of infants with birth weight less than $1500 \mathrm{gm}$. J Pediatr 1978:92:529-34

14. de Vries LS, Eken P, Dubowitz LMS. The spectrum of leukomalacia using cranial ultrasound. Behav Brain Res 1992:49:1-6

15. Prechtl HFR. The behavioral states of the newborn infant. Brain Res 1974;76:185-212

16. Task Force of the European Society of Cardiology and the North American Society of pacing and electrophysiology. Heart rate variability: standards of measurement, physiological interpretation, and clinical use. Circulation 1996;93:1043-1065

17. Bigger J, Fleiss J, Steinman R, Rolnitzky L, Kleiger R, Rottman J. Frequency domain measures of heart period variability and mortality after myocardial infarction. Circulation 1992:85:164-171

18. Malliani A, Pagani M, Furlan R, Guzetti S, Lucini D, Montano N, Cerutti S, Mela G. Individual recognition by heart rate variability of two different profiles related to posture. Circulation 1997;96:4143-4145

19. Eckberg DL. Sympathovagal balance: a critical appraisel, Circulation 1997:96:3224-3232

20. Goldberger JJ. Sympathovagal balance: how should we measure it? Am J Physiol 1999;276:H1273H1280 
21. Rosenstock EG, Cassuto Y, Zmora E. Heart rate variability in the neonate and infant: analytical methods, physiological and clinical observations. Acta Paediatr 1999;88:477-82

22. Fortrat JO. Inaccurate normal values of heart rate variability spectral analysis in newborn babies. Am J Cardiol 2002;90:346

23. Appel ML, Berger RD, Saul JP, Smith JM, Cohen RJ. Beat to beat variability in cardiovascular variables: noise or music? J Am Coll Cardiol 1989:14:1139-48

24. Giddens DP, Kitney RI. Neonatal heart rate variability and its relation to respiration. J Theor Biol 1985; 113:759-780

25. Dykes FD, Ahmann, Baldzer K, Carrigan, Kitney R, Giddens DP. Breath amplitude modulation of heart rate variability in normal full term neonates Pediatr Res 1986:20:301-308

26. Baldzer K, Dykes FD, Jones SA, Brogan M, Carrigan TA, Giddens DP. Heart rate variability analysis in full-term infants: spectral indices for study of neonatal cardiorespiratory control. Pediatr Res 1989;26:188-195

27. van Ravenswaay-Arts CMA, Hopman JCM, Kollée LAW, Stoelinga GBA, van Geijn HP. Spectral analysis of heart rate variability in spontaneously breathing very preterm infants. Acta Paediatr $1994 ; 83: 473-480$

28. Eiselt M, Curzi-Dascalova L, Clairambault J, Kauffmann F, Medigue C, Peirano P. Heart-rate variability in low-risk prematurely born infants reaching normal term: a comparison with full-term newborns. Early H Develop 1993;32:183-195

29. Veerappan S, Rosen H, Craelius W, Curcie D, Hiatt M, Hegyi T. Spectral analysis of heart rate variability in premature infants with feeding bradycardia. Pediatr Res 2000, 659-662

30. Franco P, Chabanski S, Szliwowski H, Dramaix M, Kahn A. Influence of maternal smoking on autonomic nervous system in healthy infants. Pediatr Res 2000; 47:215-220

31. Bracewell RN. The Fourier-transform and its applications. New York: McGraw-Hill, 1978

32. Jaffe RS, Fung DL. Constructing a heart rate variability analysis system. J Clin Monit 1994;10:4558

33. Akselrod S. Components of heart rate variability: basic studies. In: Malik M, Camm AJ (eds) Heart rate variability. Futura Publishing Company, New York, 1995, pp 147-163

34. Welch PD. The use of fast Fourier transform for the estimation of power spectra: a method based on time averaging over short, modified periodograms. IEEE Trans Audio and Electroacoust 1967;15:7073

35. de Boer RW, Karemaker JM, Strackee J. Relationships between short-term blood pressure fluctuations and heart rate variability in resting subjects. I: a spectral analysis approach Med Biol Eng Comput $1985 ; 23: 352-358$

36. Head GA, Lukoshkova EV, Burke SL, Malpas SC, Lambert EA, Janssen BJA. Comparing spectral and invasive estimates of baroreflex gain. IEEE Eng Med Biol 2001:20(2):43-52

37. Sagawa K. Baroreflex control of systemic arterial pressure and vascular bed. In: Shepherd JT, Abboud FM (eds.) Handbook of physiology. Section 2, volume III, part 2. Bethesda, MD. American physiological society, 1983, p 453-496 



\section{Chapter 4}

\section{Customized spectral band analysis compared with conventional Fourier analysis of heart rate variability in neonates}

Nicole A.M. de Beer, Peter Andriessen, Ralph C.M. Berendsen, S. Guid Oei, Pieter F.F. Wijn, Sidarto Bambang Oetomo

Physiological Measurements 2004(25);1385-1395

www.iop.org/journals/pmea 


\section{Abstract}

A customized filtering technique is introduced and compared with fast Fourier transformation (FFT) for analyzing heart rate variability (HRV) in neonates from shortterm recordings. FFT is classically the most commonly used spectral technique to investigate cardiovascular fluctuations. FFT requires stability of the physiological signal within a 300 -s time window that is usually analyzed in adults. Preterm infants, however, show characteristics of rapidly fluctuating heart rate and blood pressure due to an immature autonomic regulation, resulting in non-stationarity of these signals. Therefore neonatal studies use (half-overlapping or moving) windows of 64-s length within a recording time of $2-5 \mathrm{~min}$. The proposed filtering technique performs a filtering operation in the frequency range of interest before calculating the spectrum, which allows it to perform an analysis of shorter periods of only $42 \mathrm{~s}$. The frequency bands of interest are $0.04-0.15 \mathrm{~Hz}$ (low frequency, LF) and $0.4-1.5 \mathrm{~Hz}$ (high frequency, HF). Although conventional FFT analysis as well as the proposed alternative technique result in errors in the estimation of LF power, due to spectral leakage from the very low frequencies, FFT analysis is more sensitive to this effect. The response times show comparable behavior for both techniques. Applying both the methods to heart rate data obtained from a neonate before and after atropine administration (inducing a wide range of HRV), shows a very significant correlation between the two methods in estimating LF and HF power. We conclude that a customized filtering technique might be beneficial for analyzing HRV in neonates because it reduces the necessary time window for signal stability.

\section{Abbreviations}

FFT fast Fourier transform

$\mathrm{HF}$ high frequency $(0.4-1.5 \mathrm{~Hz})$

HRV heart rate variability

LF low frequency $(0.04-0.15 \mathrm{~Hz})$ 


\section{Introduction}

According to the Task force of the European Society of Cardiology and The North American Society of Pacing and electrophysiology the frequency components of heart rate variability $(\mathrm{HRV})$ are classified into 1) ultra-low frequencies $(<0.003 \mathrm{~Hz})$ that include circadian rhythm, 2) very low frequencies $(0.003-0.04 \mathrm{~Hz})$ that are supposed to be affected by temperature regulation and humoral systems ${ }^{1}, 3$ ) low frequencies (LF, $0.04-0.15 \mathrm{~Hz}$ ) that are attributed to baroreceptor activity and sensitive to changes in cardiac sympathetic and parasympathetic nerve activity, and 4) high frequencies (HF, $0.15-0.4 \mathrm{~Hz}$ ) that are synchronized to the respiratory rhythm and are primarily modulated by cardiac parasympathetic innervation ${ }^{2-5}$. According to the Task Force, the $\mathrm{LF}$ and HF components of HRV can be distinguished reliably in a spectrum calculated from short-term recordings of 300 -s length.

These Task Force recommendations, however, may not be appropriate for neonates due to a shift of the spectrum of the high frequencies ${ }^{6}$. Neonates have considerably higher respiratory rates than adults and may vary between 30 and 90 breaths per minutes, similar to 0.5 and $1.5 \mathrm{~Hz}$, respectively. Likewise, the neonatal heart rate may vary between 100 and 200 beats per minute, similar to 1.7 and $3.3 \mathrm{~Hz}$, respectively. As the neonatal heart rate and respiratory rate differ from that of the adult, neonatal studies have used different spectral divisions ${ }^{7-14}$. The cut-off points between the low frequent and high frequent band used in several neonatal studies are summarized in Table 1. Based on these studies as well as the physiological range of neonatal heart and respiratory rate, the following spectral band divisions seem appropriate for neonates: $\mathrm{LF}$ band between 0.04 and $0.15 \mathrm{~Hz}$ (reflecting baroreceptor reflex activity) and $\mathrm{HF}$ band between 0.4 and $1.5 \mathrm{~Hz}$ (reflecting the range of neonatal respiratory activity).

The use of spectral analysis techniques has been found to be useful in detecting trends and periodic changes in heart rate patterns ${ }^{15}$. Many spectral techniques rely upon the Fourier transform, or its discrete equivalent, the fast Fourier transform (FFT). The mathematical foundation of the FFT can be found elsewhere ${ }^{16}$. Conceptually, the FFT involves calculating the cross-correlations of the time signal with a series of sine and cosine functions of various frequencies. The accuracy with which high frequencies present in the signal can be resolved is determined by the sampling frequency, according to the Nyquist criterion. To obtain a good resolution of low frequencies, the FFT must be calculated over a segment of the signal that is at least as long as the longest wavelength of interest. The FFT assumes that the signal has the same spectral characteristic throughout this analysis window. When the signal spectrum is changing, 
Table 1: Low frequency (LF) and high frequency (HF) band

\begin{tabular}{llll} 
reference & population & LF(Hz) & HF(Hz) \\
\hline Aarimaa 19887 & preterm, full-term & $0.02-0.2$ & $0.2-1$ \\
Eiselt 19938 & preterm, full-term & $0.03-0.1$ & $0.4-1$ \\
Spassov 19949 & full-term & $0.03-0.1$ & $0.4-1$ \\
Ravenswaaij 199410 & preterm & $0.04-0.2$ & RSA $^{s}$ \\
Chatow 199511 & preterm, full-term & $0.02-0.2$ & $0.2-2$ \\
Mazursky 199812 & preterm & $0.02-0.15$ & $0.15-1.5$ \\
Sahni 199913 & preterm, full-term & $0.05-0.2$ & $0.5-2.0$ \\
Veerappan 200014 & preterm & $0.03-0.40$ & $0.4-1.0$ \\
\hline
\end{tabular}

$\$$, the respiratory sinus arrhythmia (RSA) was defined as a band within $\pm 0.2 \mathrm{~Hz}$ of the respiratory frequency obtained from peak amplitude in the respiratory power spectrum.

the resulting Fourier spectrum will be an average spectrum over the entire analysis window. As a consequence, short periods of HF activity in the signal will be visible in the spectrum (provided they are strong enough), but it will not be possible to determine at which time instant in the analysis window the HF activity occurred.

In physiological conditions, signal stability is difficult to ensure. Notably preterm infants show characteristics of rapidly fluctuating heart rate and blood pressure due to an immature autonomic regulation, resulting in non-stationarity of these signals. Therefore, a compromise has to be chosen between the lowest frequency of interest, which determines a minimum window length, and the required accuracy for establishing the occurrence of HF activity, which determines a maximum window length. Alternative analysis methods such as wavelet transform analysis circumvent this compromise by calculating different parts of the spectrum using different window lengths. In fetuses the wavelet transform has been applied to study the rapid fluctuating autonomic state ${ }^{17}$.

The aim of the present study is to introduce a customized filtering technique applicable to neonates, to cope with the compromise between LF $(0.04-0.15 \mathrm{~Hz})$ frequency resolution and $\mathrm{HF}(0.4-1.5 \mathrm{~Hz})$ time resolution. This technique is based on the assumption that for characterization of autonomic cardiovascular regulation, only specific frequency bands are of interest and thus only specific frequency bands need to be analyzed. The classical FFT as well as the customized technique will be used to assess LF and HF fluctuations of the cardiovascular control system in neonates. 
First, the current implementation of conventional FFT analysis is described, followed by a description of the customized filtering technique. The theoretical properties of both techniques are described, and evaluated on simulated signals. Finally, both techniques are compared on actual heart rate data obtained in neonates.

\section{Materials and methods}

\section{Neonatal data acquisition}

Neonatal data acquisition took place at the neonatal intensive care unit (NICU) of our hospital. In the NICU, each patient monitor is linked with a central data acquisition system for both on-line and off-line analysis. This enables the possibility of building up a database of neonatal HRV signals. Signals from this database were used for the present study.

A bipolar chest lead of the surface ECG was recorded by a Hewlett Packard neonatal monitor, type Merlin (Waltham, MA, USA). The analog ECG signals were transferred to the central data acquisition system, where the signals were digitized at a sampling frequency of $512 \mathrm{~Hz}$. The R-peaks were detected from the ECG using a second-order polynomial fit. Without fitting, the maximum error in establishing R-peak location is half the sampling interval, i.e., $0.98 \mathrm{~ms}$. Because of the polynomial fitting, a higher time resolution can be achieved. Previous work has empirically established that polynomial fitting results in a 6-fold increase in accuracy of R-peak detection, i.e., a time resolution of $0.16 \mathrm{~ms}^{18}$. The thus-created unevenly spaced $\mathrm{R}-\mathrm{R}$ interval sequence was resampled using a boxcar window at $4 \mathrm{~Hz}^{19}$ to obtain an equidistantly-spaced time series. R-R artifacts data were identified if the R-R interval was less than $200 \mathrm{~ms}$ or more than 500 $\mathrm{ms}$, or when the difference between consecutive R-R intervals was larger than $12 \%$. Missing R-R intervals were linearly interpolated. The mean R-R interval was calculated and subtracted from all data points to remove the DC component. These preprocessed evenly spaced R-R interval series constitute the basis of analyzing HRV in neonates. We refer to an earlier paper for further details of neonatal data acquisition of physiological signals $^{20}$.

The boxcar resampling introduces a distortion into the frequency spectrum of the R-R series. To correct for this distortion, the power density spectrum of the signal is divided by the square of a sinc function, which is the square of the FFT of a rectangular time window. In the customized filtering technique, this sinc function is applied to the calculated filter spectra. 


\section{Spectral analysis}

\section{Fourier analysis}

To calculate the power density spectrum of the resampled R-R series, a 256-point (64 s) moving FFT is used, providing a spectrum every $0.25 \mathrm{~s}$. Before FFT, drift in the signal is corrected by subtracting a linear fit based on the minimum square error criterion. This correction is necessary to reduce the effect of spectral leakage, especially of the frequency components below the LF band. In addition, a Parzen window is applied to further reduce the effect of spectral leakage. The power in both the LF and HF bands is calculated by summing the power spectrum over the frequency range defined by the band. For the LF band, this range is 0.04 to $0.15 \mathrm{~Hz}$, for the HF band it is 0.4 to $1.5 \mathrm{~Hz}$. Because the analysis is applied on a 256-point sequence with a sampling frequency of $4 \mathrm{~Hz}$, each frequency bin in the power spectrum is $0.0156 \mathrm{~Hz}$ wide. The LF band thus corresponds to bins 4 through 10, while the HF band corresponds to bins 27 through 96 .

\section{Customized filtering}

In the FFT analysis described above, only portions of the spectrum are used to quantify LF and HF fluctuations in heart rate. Because the entire spectrum needs to be calculated, the minimum length of the signal segment to be analyzed is determined by the lowest frequency of interest. As a result, the HF band is analyzed with the same frequency resolution, even though a much shorter segment would suffice for analysis of the HF band. Therefore, in the current analysis, we perform separate analyses of the LF and HF bands by filtering the R-R series in these two frequency bands. The power of the resulting filtered signals then represents the power in the respective bands. This approach is valid when the definition of the LF and HF ranges is stable, i.e., the dominant activity does not change to frequencies outside of the defined bands. For the LF range this has been established elsewhere ${ }^{21}$. Because definition of the HF band depends on the respiration frequency, the HF band is chosen to include a range of respiration frequencies that are expected to occur in neonates.

Design of the LF and HF filters is achieved by the window method. Ideally, for each band the ideal spectrum of the filter would be a rectangle covering the desired frequency range. The spectrum $\mathrm{H}_{\mathrm{LF}}(\mathrm{f})$ of the function used to estimate the $\mathrm{LF}$ band consists of zeroes outside the LF band and ones (multiplied by a sinc function for boxcar correction) inside the LF band. For the HF band the same method is used, this time filling the HF band with ones multiplied by a sinc function. Subsequent inverse FFT of $\mathrm{H}_{\mathrm{LF}}(f)$ and $\mathrm{H}_{\mathrm{HF}}(f)$ results in the impulse responses $h_{\mathrm{LF}}(k)$ and $h_{\mathrm{HF}}(k)$ of the filters. 
For the present application, using the shortest possible filter is an important prerequisite. To determine the number of points to be used in the filter, the LF filter was applied to a simulated signal of 200-s duration, with a flat spectrum over the range 0$0.2 \mathrm{~Hz}$. Ideally, the spectrum of the filtered signal should be 1 in the range $0.04-0.15 \mathrm{~Hz}$ and zero outside that range. The squared differences between this ideal spectrum and the actually achieved spectrum by applying the LF filter were calculated and summed. This process was repeated for a range of LF filter lengths. The resulting squared differences are presented in Figure 1. As can be seen in this figure the smallest difference is found for 168 points ( $42 \mathrm{~s}$ ). Using a length of 90 points ( $22.5 \mathrm{~s})$ gives a local minimum and is another possibility. A similar approach could have been used for designing the HF filter. Instead, we chose to have the same filter length for HF and LF analysis, because they will be analyzed together and compared to each other in future studies.

The calculated transfer functions of both filter lengths for the LF band are compared with the FFT transfer. This comparison is shown in Figure 2. Both filter lengths result in a good estimation of the desired transfer function, yet the 90-point filter shows a relatively large difference for the frequencies below the LF band. This will result in spectral leakage of signal from the lowest frequencies into the LF band, which may result in an overestimation of the LF power. Because all analyzed signals have a major frequency component below LF a systematic overestimation will occur. Therefore, we chose to use the 168-point filter, even though time resolution will be slightly worse than for the 90 -point filter.

Using the derived filters, $y \operatorname{LF}(n)$ and $y H F(n)$ are calculated by performing a convolution of the time signal with the impulse responses $h_{\mathrm{LF}}(k)$ and $h_{\mathrm{HF}}(k)$, respectively, thus avoiding a transformation to the frequency domain. The power of the resulting signals as a function of time is calculated to give the LF and HF powers, respectively.

\section{Comparison of properties of the analysis techniques}

\section{Response time comparison}

As already mentioned above, the most important property of the analysis technique is the length of the analysis window. Other important properties are errors in power estimation due to white noise, spectral leakage from below the LF band, and the response time to changing frequency content. The effect of spectral leakage was already 


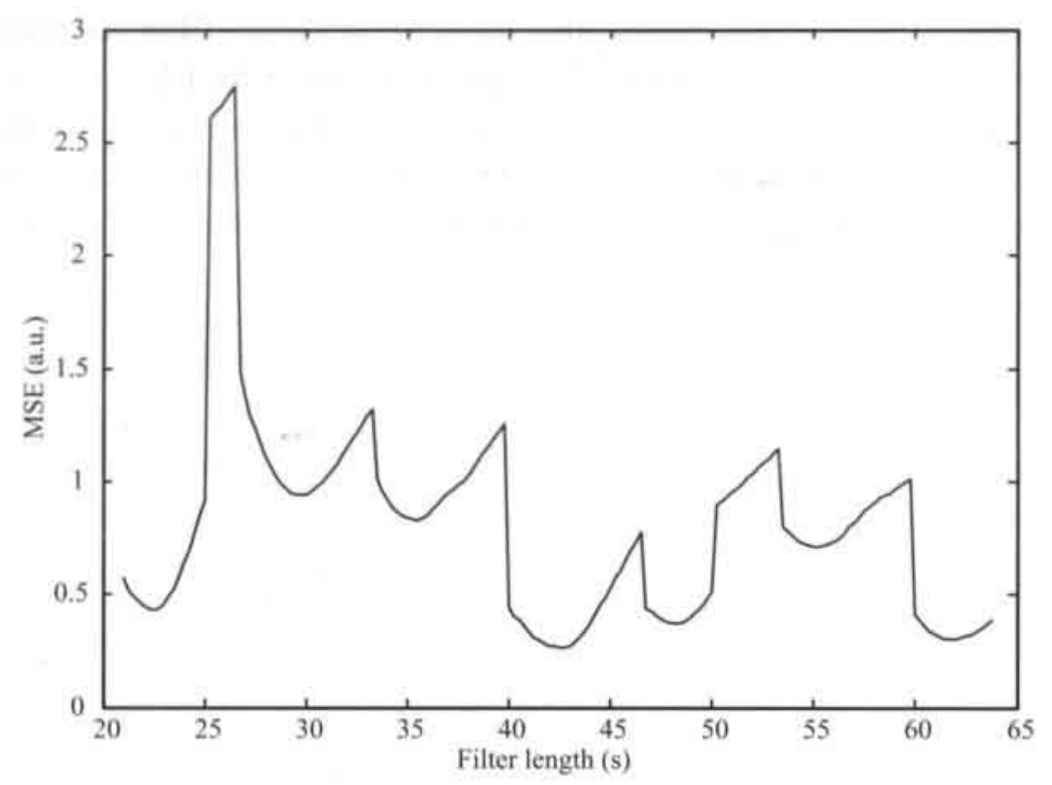

Figure 1. The mean squared error (MSE) between the desired transfer and the proposed filter transfer function is expressed as function of LF filter length.

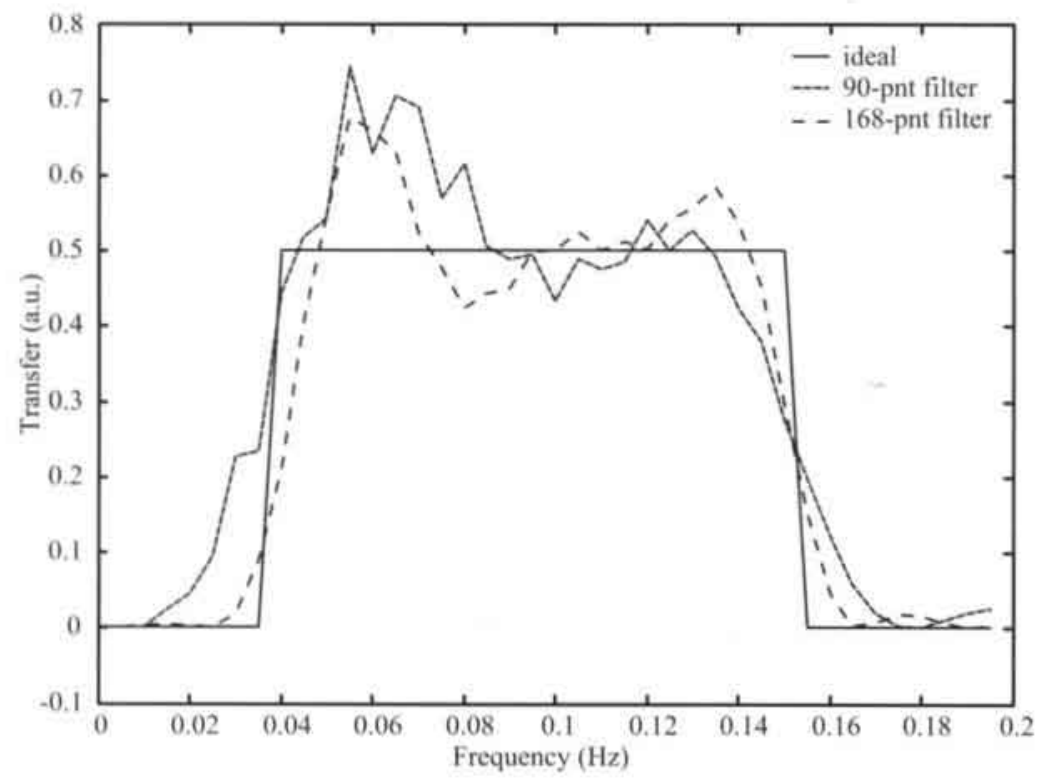

Figure 2. Comparison of the desired transfer function and the transfer functions calculated with the filters of length 90 and 168 points for the LF band. 
discussed in the previous section, as this was an important consideration for choosing the appropriate filter length. The response time to changing frequency content is studied using simulated data, consisting of a zero signal for $70 \mathrm{~s}$, followed by a sinusoid also of 70-s duration, with a frequency of $0.1 \mathrm{~Hz}$ for analyzing the LF response, or a frequency of $1.0 \mathrm{~Hz}$ for analyzing the $\mathrm{HF}$ response.

\section{Noise level comparison}

As for many physiological data series, the HRV signal can be assumed to be the sum of definite physiological signal components plus Gaussian white noise. This white noise manifests itself in the spectrum as an added noise level over the entire spectrum. When calculating the LF and HF components of the HRV spectrum, a part of these components will be due to noise. To assess the magnitude of this noise, both the conventional FFT and the customized technique are applied to a simulated signal with a flat power spectrum over the entire analyzed range.

\section{Comparison on neonatal data}

Actual heart rate data were used to compare the results of FFT analysis and customized filtering. The data were obtained from a neonate before and after atropine administration. Atropine, as a muscarinic receptor antagonist, exerts its influence on postganglionic receptors of the parasympathetic system at the sinoatrial node of the heart. Because LF fluctuations are associated with the orthosympathetic as well as the parasympathetic system, and HF fluctuations are associated with only parasympathetic activity, atropine decreases the LF and HF energy content of HRV. Atropine modulates the parasympathetic tone over a wide range, and thus HRV will considerably vary before and after atropine. This wide induced range of variability is of interest for our analysis. We analyzed HRV before $(15 \mathrm{~min}$ ) and after atropine (15 min). The LF and HF results of FFT analysis during this 30 -min period are compared with the results of the customized filtering analysis.

Statistical analysis: The Pearson correlation coefficient was calculated between both methods and linear regression analysis using the method of least squares (intercept constant $a$, regression coefficient $b, 95 \%$ confidence interval) was performed for the spectral power values. Bias in LF and HF power values was calculated as the mean of difference between conventional FFT and customized spectral band analysis, with its $95 \%$ limits of agreement ${ }^{22}$. To ensure that all data points are independent, only data points with 64-s intervals are used in this calculation, starting at $32 \mathrm{~s}$. Statistical significance was accepted at $\mathrm{p}<0.05$. 


\section{Results}

\section{Response time comparison}

Figure 3 presents the result of the response time comparison. The upper panel shows the result of the comparison on a change in LF content. The lower panel shows the result of the comparison on a change of HF content. The results demonstrate that the FFT analysis responds almost as fast as the 42 -s filter analysis. The 21-s filter analysis responds faster than FFT analysis.

\section{Noise level comparison}

The noise level in the HRV measurements, estimated from the parts in the power density spectrum where apparently no physiological information is present, typically has a power of about $0.16 \mathrm{~ms}^{2}$. Applying both conventional FFT analysis and the customized filtering technique to a simulated signal with a flat power spectrum of 0.16 $\mathrm{ms}^{2}$ results in estimation errors of $0.012 \mathrm{~ms}^{2}$ and $0.14 \mathrm{~ms}^{2}$ for LF and HF power obtained from FFT analysis ( $7.3 \%$ and $91 \%$ of HRV noise power, respectively), and $0.009 \mathrm{~ms}^{2}$ and $0.15 \mathrm{~ms}^{2}$ for LF and HF power obtained with the filhering technique (5.6\% and $91 \%$ of HRV noise power, respectively) The relatively high noise contribution in HF power is partly due to the applied sinc function correction, which emphasizes high frequencies, and partly to the fact that the analyzed HF range occupies a significant part of the power spectrum. i.e.. $(1.5-0.4) / 2=55 \%$.

\section{Neonatal data}

In Figure 4 the 30-min recording of heart rate is shown: 15 min baseline (before atropine) and 15 min after atropine. Six artifacts were identified in the R-R tracing, with lengths of 4,5 and 6 consecutive heart beats. These artifacts were linearly interpolated. In Figure 4, the vertical dotted line at time $900 \mathrm{~s}$ indicates atropine administration. After atropine heart rate increases and variability decreases. The results of the FFT and customized filtering technique to estimate LF and $\mathrm{HF}$ power of heart rate are shown in Figure 5. Note that after atropine administration the LF spectral power is markedly decreased. As can be seen from the diagrams both methods show good agreement, in a wide range of spectral energy, for both the LF and HF power. The values of the regression equation customized filter value $=a+b$. FFT value are displayed in Table 2. The results show that the two methods correlate very well with each other: the $95 \%$ confidence interval for the intercept constant $a$ includes zero and the $95 \%$ confidence interval for the regression coefficient $b$ includes one, both for $\mathrm{LF}$ and for $\mathrm{HF}$ power. The mean of spectral power differences between FFT and customized spectral band analysis was 6 (95\% limits of agreement: -29 to 41$) \mathrm{ms}^{2}$ for LF values and 0 (95\% limits of 
agreement: -3 to 4$) \mathrm{ms}^{2}$ for HF values. There is a slight (not significant) overestimation of LF and HF power by the customized filtering technique.
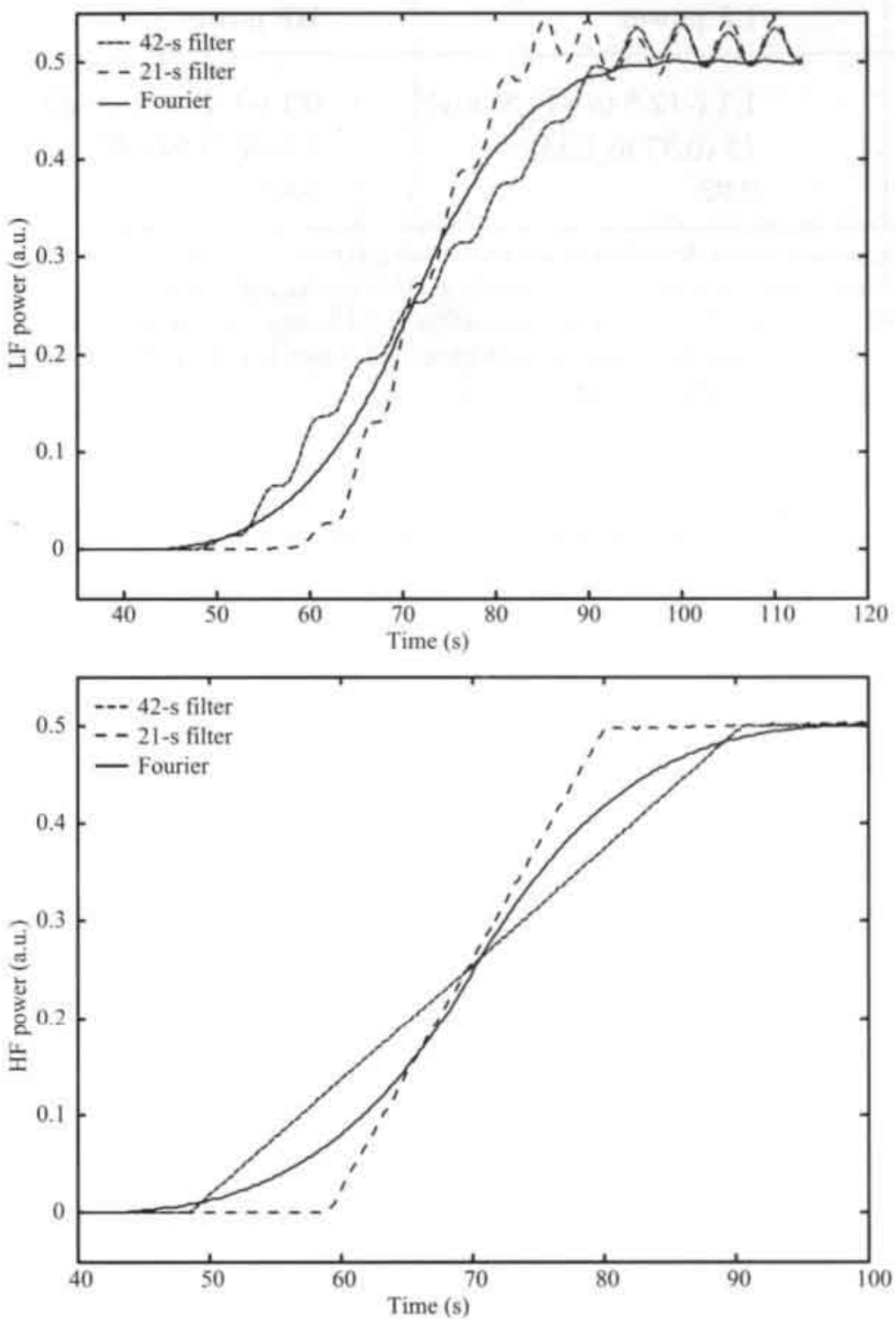

Figure 3. Response curves on a stepwise change at $t=70 \mathrm{~s}$ in power contents of the signal for $42-\mathrm{s}$ and 21-s filter lengths, and Fourier analysis. The results for the LF band are presented in the upper panel, for the $\mathrm{HF}$ band in the lower panel. Note that although all methods are equally capable of detecting the (average) instant of a change in frequency, for a real-time presentation of the results, Fourier analysis results will show a $64-\mathrm{s}$ delay, while the results of the customized filtering analysis will show $42-\mathrm{s}$ or $21-\mathrm{s}$ delays, respectively. 
Table 2: Correlation of LF and HF power between the two methods

\section{LF power}

$\begin{array}{lll}a & 1.1(-12.5 \text { to }+14.8) \mathrm{ms}^{2} & 0.1(-1.9 \text { to }+2.2) \mathrm{ms}^{2} \\ b & 15(0.97 \text { to } 1.33) & 1.0(0.75 \text { to } 1.32) \\ r & 0.93 & 0.82\end{array}$

The linear regression line is described by the regression equation customized filter value $=a+b \cdot F F T$ value, with its $95 \%$ confidence interval between brackets. Both for LF and HF power, the $95 \%$ confidence interval for the intercept constant (a) includes zero, and the $95 \%$ confidence interval for the regression coefficient $(b)$ includes 1 . The correlation coefficients $(r)$ are significant at $\mathrm{p}$ values $<0.001$.

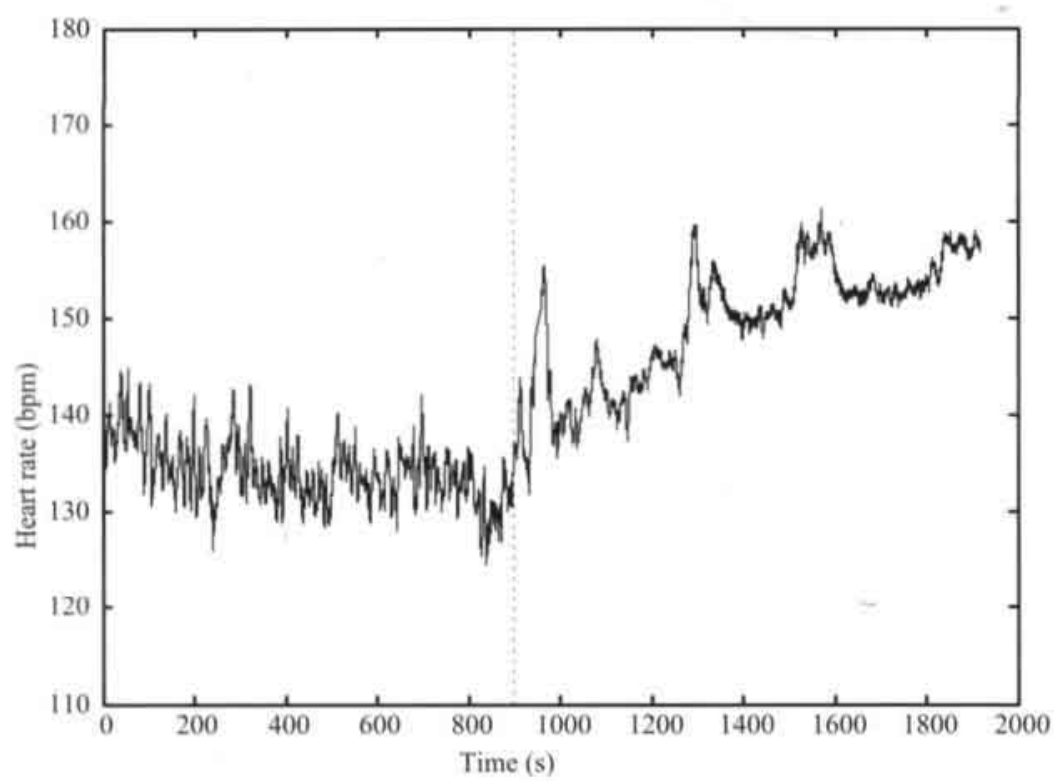

Figure 4. Heart rate series (beats per minute, bpm) obtained from a newborn. Atropine administration is indicated by the vertical dotted line at $900 \mathrm{~s}$. Note the increase in heart rate and the decrease in variability within $5 \mathrm{~min}$ of atropine administration. 

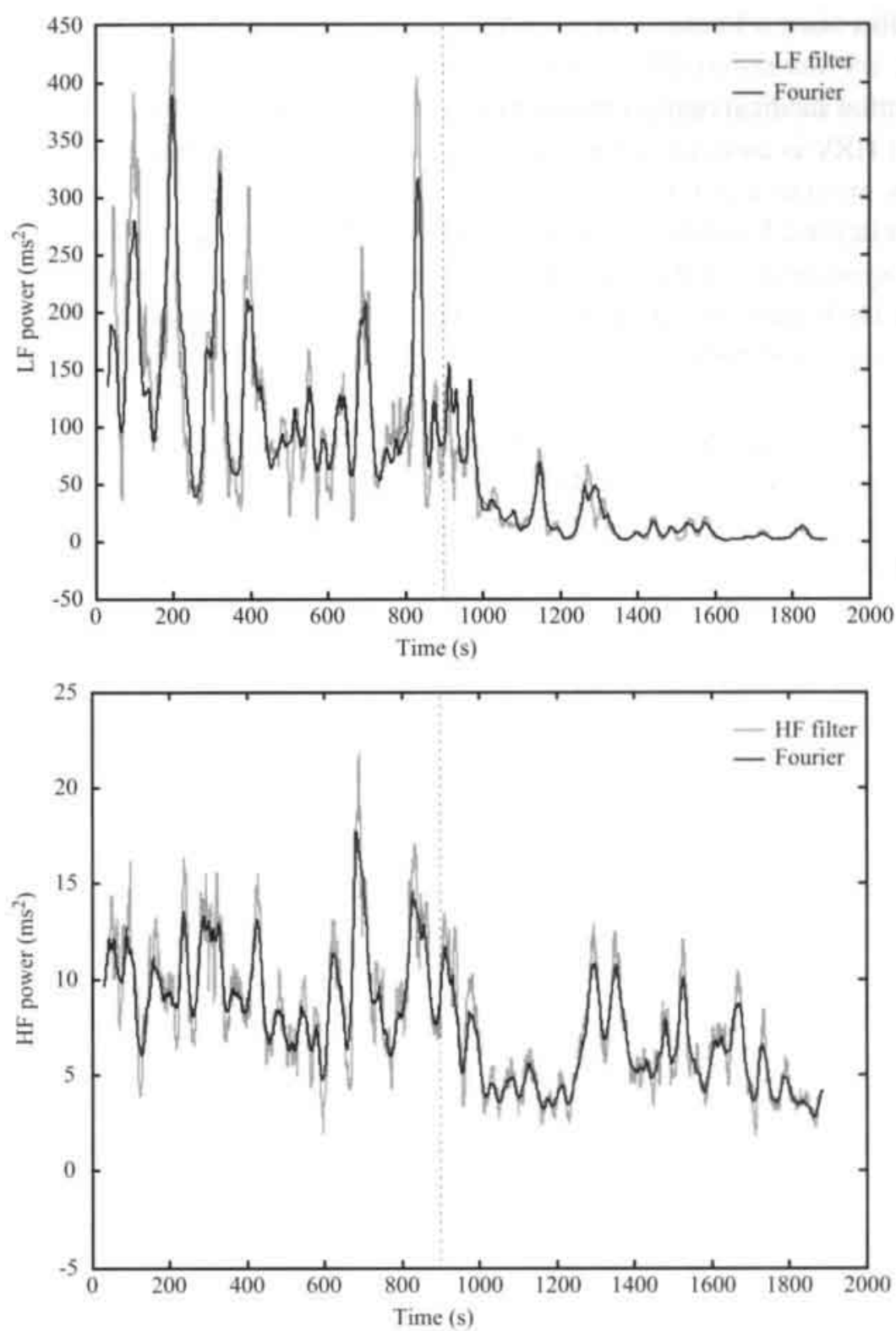

Figure 5. Power estimations of the Fourier and the filtering method of heart rate series shown in Figure 4. Atropine administration is indicated by the vertical dotted line at $900 \mathrm{~s}$. Top: LF band, bottom: HF band. Both the Fourier and the filtering estimates show comparable behavior. 


\section{Discussion}

The potential medical application of monitoring HRV arises from the observation that abnormal HRV is associated with neonatal morbidity and mortality. Monitoring HRV may thus provide a non-invasive diagnostic tool of clinically important diseases. A reduction in the LF or HF component of $\mathrm{HRV}$ is associated with an active respiratory distress syndrome ${ }^{7}$, birth asphyxia and intra-ventricular hemorrhage ${ }^{23,24}$. With recovery, HRV increases. In addition, abnormal heart rate characteristics may precede neonatal sepsis and death 25,26 .

In contrast to human adults, signal stability is difficult to ensure because preterm infants show characteristics of rapidly fluctuating heart rate and blood pressure due to an immature autonomic cardiovascular regulation. We present an alternative technique for spectral band analysis of heart rate data, with the purpose of reducing the influence of non-stationarities in the analyzed signals. This is achieved by first filtering the analyzed signal in the spectral region of interest, and subsequently calculating the power of the filtered signal, instead of first calculating the complete spectrum in FFT analysis.

The results show that the two methods correlate significantly for the LF and HF frequency bands. An important difference between the two methods is the effect of spectral leakage, i.e., the influence of very low frequency components on the results. Noise level comparison shows that FFT analysis is slightly more sensitive to LF noise than customized filtering. The neonatal data show greater bias and wider limits of agreement for the LF than for the HF band. These effects are due to the effect of spectral leakage. For the customized filtering analysis, spectral leakage is not an issue because the major part of very low frequencies is filtered out, thus not affecting the results.

The results also show that both methods are quite sensitive to HF noise. However, with the physiological signals analyzed here this will not be a problem, because the HF activity of interest is usually much larger than the amount of HF noise intrinsically present in the signal ${ }^{20}$.

An important motivation for this study was the need to track the effect of nonstationarities on frequency analysis of heart rate signals. The results show that the capability of the proposed technique to do this is limited by the requirement to include $\mathrm{LF}$ power in the analysis. For HF power, it would be possible to give a better definition of changing frequency. Due to the requirement to be able to analyze LF power as well, however, the minimum window length remains rather large, resulting in a smoothing of 
HF power over the analysis window. This is again the dilemma of a trade-off between the desired resolution in detecting short-lasting changes in HF power and the detection of changes in LF power. Effectively, this trade-off is determined by the definition of the frequency regions of interest.

Other methods exist to analyze LF and HF power in the presence of non-stationarities. Among these are wavelet analysis and time-frequency analysis $21,27,28$. Compared with our approach, these techniques have the advantage that they do not assume a fixed frequency range of interest, as is done in the analysis described in this work. A disadvantage of wavelet analysis is the need to use the same wavelet shape over all frequency ranges to be analyzed. This requires a major effort for properly choosing the appropriate wavelet. Time-frequency analysis, although very promising for research purposes, suffers from so-called cross-terms, which introduce spurious power in the presence of multiple frequencies. Since the frequency ranges of interest were already established in the present study, a relatively simple approach as in the present study can be used, which has the advantage of being straightforward to implement.

An interesting result is the fact that there is only a very small difference in the response of both methods to changing frequency content. This is caused by the application of a Parzen window to reduce spectral leakage in the FFT analysis. For the customized filtering analysis this windowing is not necessary, because the effect of very low frequencies is already removed by the filter. Due to the Parzen window, however, FFT analysis is also better able to define fast changes in frequency content of the analyzed signal. Note, however, that FFT analysis still suffers from a longer delay in presentation of the results (64 s) when compared with the customized filtering technique (42 or $21 \mathrm{~s})$ but for clinical monitoring applications this is essentially not a problem.

It should be noted that the described definition of the applied filters is rather crude. Using standard filter design methodology, more sophisticated filters could have been designed. Nevertheless, the results show that even with the presented filters, the proposed analysis technique results in less sensitivity to LF noise, but not in a better definition of the response to changing frequency content. This is because the application of a Parzen window already results in a quite optimal performance of the FFT analysis in the presence of non-stationarities.

In summary, the proposed alternative spectral analysis technique is adequate in estimating LF and HF power of neonatal HRV. The proposed technique results in less sensitivity to LF noise than conventional Fourier analysis. The sensitivity of the 
proposed technique to non-stationarities in other frequency ranges is, however, only marginally affected. We conclude that a customized filtering technique might be beneficial for analyzing HRV in neonates because it reduces the necessary time window for signal stability. 


\section{References}

1. Taylor JA, Carr DL, Myers CW, Eckberg DL. Mechanisms underlying very-low-frequency R-Rinterval oscillations in humans. Circulation 1998; 98:547-555

2. Task Force of the European Society of Cardiology and the North American Society of Pacing and Electrophysiology. Heart rate variability: Standards of measurement, physiological interpretation and clinical use. Circulation Electrophysiology 1996; 93(5):1043-1065

3. Malpas SC. Neural influences on cardiovascular variability: possibilities and pitfalls. Am J Physiol 2002;282:H6-H20

4. Stauss HM. Heart rate variability. Am J Physiol 2003;285:R927-R931

5. Brown TE, Beightol LA, Koh J, Eckberg DL. Important influence of respiration on human R-R interval power spectra is largely ignored. J Appl Physiol 1993; 75(5):2310-2317

6. Rosenstock EG, Cassuto Y, Zmora E. Heart rate variability in the neonate and infant: analytical methods, physiological and clinical observations. Acta Paediatr 1999;88:477-482

7. Aärimaa T, Oja R, Antila K, Välimäki I. Interaction of heart rate and respiration in newborn babies. Pediatr Res 1988;24:745-750

8. Eiselt M, Curzi-Dascalova L, Clairambault J, Kauffmann F, Medigue C, Peirano P. Heart-rate variability in low-risk prematurely born infants reaching normal term: a comparison with full-term newborns. Early H Develop 1993;32:183-195

9. Spassov L, Curzi-Dascalova L, Clairambault J, Kauffmann F, Eiselt M, Medigue C, Peirano P. Heart rate and heart rate variability during sleep in small-for-gestational age newborns. Pediatr Research 1994;35:500-505

10. van Ravenswaay-Arts CMA, Hopman JCM, Kollée LAW, Stoelinga GBA, van Geijn HP. Spectral analysis of heart rate variability in spontaneously breathing very preterm infants. Acta Paediatr 1994;83:473-480

11. Chatow U, Davidson S, Reichman BL, Akselrod S. Development and maturation of the autonomic nervous system in premature and full-term infants using spectral analysis of heart rate fluctuations. Pediatr Res 1995;37:294-302

12. Mazursky JE, Birkett CL, Bedell KA. Ben-Haim SA. Segar JL. Development of baroreflex influences on heart rate variability in preterm infants. Early Hum Dev 1998:53:37-52

13. Sahni R, Schulze KF, Kashyap S, Ohira-Kist K. Fifer WP, Myers MM. Postural differences in cardiac dynamics during quiet and active sleep in low birth infants. Acta Paediatr 1999:88:1396-1401

14. Veerappan S, Rosen H, Craelius W, Curcie D, Hiatt M. Hegyi T. Spectral analysis of heart rate variability in premature infants with feeding bradycardia. Pediatr Res 2000, 659-662

15. Jaffe RS, Fung DL. Constructing a heart-rate variability analysis system J Clin Monit. 1994 Jan:10(1):45-58

16. Bracewell RN. The Fourier-transform and its applications. New York: McGraw-Hill, 1978

17. Kimura Y, Okamura K, Wantanbe T, Yaegashi N, Uehara S, Yajima A. Time-frequency analysis of fetal heartbeat fluctuation using wavelet transform. Am J Physiol 1998:275:H1993-H1999

18. de Jong W. Blood pressure variability in neonates: with a special focus on signal acquisition and signal processing. PhD Thesis 2000, Eindhoven University of Technology, Eindhoven, The Netherlands

19. Berger RD, Akselrod S, Gordon D, Cohen RJ. An efficient algorithm for spectral analysis of heartrate variability. IEEE Tran. Biomed. Eng 1986:33:900-914

20. Andriessen P, Koolen AM, Berendsen RC, Wijn PF, ten Broeke ED, Oei SG, Blanco CE. Cardiovascular fluctuations and transfer function analysis in stable preterm infants. Pediatr Res 2003;53(1):89-97 
21. Lotric MB, Stefanovska A, Stajer D, Urbancic-Rovan V. Spectral components of heart rate variability determined by wavelet analyis. Physiol Meas 2000, 21(4): 441-457

22. Bland JM. Altman DG. Statistical methods for assessing agreement between two methods of clinical measurement. Lancet 1986;1:307-310

23. Divon MY, Winkler H, Yeh SY, Platt LD, Langer O, Merkatz IR. Diminished respiratory sinus arrhythmia in asphyxiated term infants. Am J Obstet Gynecol 1986:155:1263-1266

24. Prietsch V, Knoepke U, Obladen M. Continuous monitoring of heart rate variability in preterm infants. Early Hum Dev 1994;37:117-131

25. Griffin MP, Moorman JR. Toward the early diagnosis of neonatal sepsis and sepsis-like illness using novel heart rate analysis. Pediatrics 2001;107:97-104

26. Griffin MP, O'Shea TM, Bissonette EA, Harrell Jr FE, Lake DE, Moorman JR. Abnormal heart rate characteristics are associated with neonatal mortality. Pediatr Res 2004;55:782-788

27. Wiklund U, Akay M, Niklasson U. Short-term analysis of heart-rate variability by adapted wavelet transforms: Methods for characterizing autonomic nervous system modulation of cardiovascular activity. IEEE Eng Med Biol Mag 1997; 16(5):113-8

28. Pichot V, Gaspoz JM, Molliex S, Antoniadis A, Busso T, Roche F, Costes F, Quintin L, Lacour JR, Barthélémy JC. Wavelet transform to quantify heart rate variability and to assess its instantaneous changes. J Appl Physiol 1999;86:1081-1091 


\section{Chapter 5}

\section{Noninvasive assessment of blood pressure variability in preterm infants}

Peter Andriessen, Rick L.M. Schoffelen, Ralph C.M Berendsen, Nicole A.M. de Beer,

S. Guid Oei, Pieter F.F Wijn, Carlos E. Blanco

Pediatric Research 2004;55:220-223

www.pedresearch.org/content/vol55/issue2/ 


\section{Abstract}

The feasibility of measuring blood pressure (BP) variability by a noninvasive beat-tobeat finger arterial BP device (Finapres) was assessed in preterm infants. By application of the finger cuff around the infant's wrist, time and frequency domain (spectral power) analysis of noninvasive beat-to-beat BP signals were compared with intra-arterial measurements. A fast Fourier transform was used to compute the spectral power density from 128 -s periods. The low frequency band (LF, 0.04-0.15 Hz) is partly associated with baroreflex activity. The high frequency band $(\mathrm{HF}, 0.4-1.5 \mathrm{~Hz})$ is associated with respiratory activity. In eight subjects above $1000 \mathrm{~g}$ reliable signals could be obtained. We observed a high correlation between noninvasive and intra-arterial beat-to-beat systolic $\mathrm{BP}$ values (mean r-value $\pm \mathrm{SD}, 0.87 \pm 0.11$ ), with a gain close to 1 (mean gain $\pm \mathrm{SD}, 1.0 \pm 0.4 \mathrm{mmHg} / \mathrm{mmHg}$ ). Finapres estimated beat-to-beat systolic BP changes more accurately than diastolic values. We found a very high amount of linear coupling, expressed as coherence function, between the power spectra of noninvasive and intraarterial systolic BP measurements. For systolic BP, the (pooled) group mean \pm SEM coherence values were $0.93 \pm 0.00$ and $0.91 \pm 0.01$ for LF and HF fluctuations, respectively (NS). The wrist method of Finapres in neonates has limited value in estimating absolute BP but is useful in clinical research situation, where identification of beat-to-beat changes in systolic BP is more important. Finapres provides a noninvasive tool for investigating autonomic cardiovascular regulation (baroreflex sensitivity, spectral analysis of BP fluctuations) in neonates.

\section{Abbreviations}

BP blood pressure

DBP diastolic blood pressure

$\mathrm{HF}$ high frequency $(0.4-1.5 \mathrm{~Hz})$

LF low frequency $(0.04-0.15 \mathrm{~Hz})$

SBP systolic blood pressure 


\section{Introduction}

Finapres (FINger Arterial PRESsure) is a noninvasive blood pressure (BP) method, based on the volume-clamp principle ${ }^{1}$. The device is based on a photoplethysmographic system applied to the finger and provides a continuous beat-to-beat waveform. Several studies of Finapres have been reported and have shown good agreement with intraarterial measured BP, in both adults and children ${ }^{2-4}$. Despite abundant literature about the application of Finapres in adults, very little is known in neonates. Drouin et al. published in 1997 about the accuracy of Finapres measurements in the neonate 5 . Because no appropriately sized cuff exists for neonates, Drouin placed the finger cuff around the wrist of the baby. Absolute Finapres BP values were compared with intraarterial $\mathrm{BP}$ for selected periods of $5 \mathrm{~s}$, and it was concluded that both methods gave similar results. However, no information was given about the accuracy of measuring BP fluctuations over longer periods of time. We do know from studies in adults, however, that Finapres may overestimate the low frequency fluctuation ${ }^{6}$. The reliability of Finapres to produce frequency domain analysis in neonates has not been assessed. The aim of this study was to determine 1) the accuracy of Finapres in measuring BP absolute values and beat-to-beat changes, and 2) the accuracy of Finapres in studying $\mathrm{BP}$ variability by frequency domain analysis.

\section{Methods}

Subjects: The study was performed in 12 preterm infants (gestational age, 26-33 wk; birth weight, $750-2215 \mathrm{~g}$ ) whose intensive care management required an umbilical arterial catheter. Informed consent was obtained from the parents of each subject. The local Ethics Committee approved the study.

Data acquisition: The Finapres device (model 5, TNO Institute of Applied Physics, Biomedical Instrumentation, Amsterdam, The Netherlands) consists of a finger BP cuff with a built-in infra-red sensitive photocell; a pressure manometer and an automatic unit for cuff inflation; and a monitor with digital display of SBP and DBP, and heart rate. After self-calibration, a servomechanism pneumatically attached to a noncompliant cuff that fits around the finger continuously adjusts air pressure in the cuff to maintain a constant transmural pressure in the digital arteries. This is achieved by continuously monitoring a photoelectric signal that passes through the finger. Absolute pressure values are determined at the time of self-calibration by a proprietary algorithm. After calibration, the servosystem tries to nullify the pulsations. When this 
occurs, the transmural pressure is zero and an approximately linear relation exists between intra-arterial pressure and transmittance ${ }^{2}$.

Arterial BP was measured invasively through a 4 French catheter placed earlier in the lower aortic position for routine monitoring of vital functions and intensive care management. A $0.5 \mathrm{ml} / \mathrm{h}$ infusion of heparinized physiological saline solution was continuously flushed through the catheter. The smallest (white) cuff supplied with the Finapres device was placed around the left wrist. After self-calibration (Physiocal), 10min recordings (servo control off) were obtained in each subject. During the procedure of Finapres measurement, the skin colored temporarily dark red to blue during the insufflation of the cuff, which restored to normal immediately after desufflation of the cuff. None of the babies sustained vascular damage or discomfort from the use of the wrist cuff during the measurements.

Data analysis: For each subject, we selected a period of $128 \mathrm{~s}$ (consisting of 3 halfoverlapping segments of $64 \mathrm{~s}$ ) for further data analysis. A 2-min period was chosen as a compromise between the need for signal stability and risk on movement artifacts from the wrist cuff.

Both BP signals were sampled at $128 \mathrm{~Hz}$ and stored on a data acquisition system. Systolic BP (SBP) and diastolic BP (DBP) were identified from "peak" detection of the BP signal in 128-s period. This procedure resulted in an unevenly spaced "systogram" and "diastogram". These unevenly time-spaced series were resampled into equidistantly spaced series using a boxcar window at $4 \mathrm{~Hz}^{7}$. Spectral leakage was reduced by subtracting the direct current variability and by multiplying with a triangular Parzen windowing ${ }^{8}$. Filter correction removed unwanted side effects of resampling and Parzen windowing. Each 128-s period of preprocessed evenly spaced BP series was subdivided in 3 half-overlapping 64-s ( 256 points) segments. A fast Fourier transform was used to compute the power spectral density. A mean power spectrum was derived from 3 halfoverlapping 64-s spectral density functions to increase accuracy. The total power was computed for the frequency band between 0.04 and $1.5 \mathrm{~Hz}$. The value for power was expressed in square millimeter of mercury. The low frequency band (LF band), partly associated with baroreflex activity, was defined between 0.04 and $0.15 \mathrm{~Hz}$. The high frequency band ( $\mathrm{HF}$ band), associated with respiratory activity, was defined between 0.4 and $1.5 \mathrm{~Hz}^{9}$. The maximum (Nyquist) frequency is $1.5 \mathrm{~Hz}$, assuming the heart rate to be $<3 \mathrm{~Hz}$ or $180 \mathrm{bpm}$.

Statistical analysis: Bias in absolute and beat-to-beat SBP and DBP values was calculated as the mean of difference between noninvasive and intra-arterial BP values, with its $95 \%$ limits of agreement ${ }^{10}$. The SD of the bias in these signals was calculated 
in each patient as an index of intrapatient variation. BP gain and offset were calculated by linear regression between non-invasive and arterial values of SBP and DBP: (beatto-beat) $\mathrm{BP}_{\text {noninvasive }}=$ gain $\cdot$ (beat-to-beat) $\mathrm{BP}_{\text {invasive }}+$ offset $^{11}$.

In addition we estimated, in frequency domain, the amount of coupling (squared coherence function) between the two power spectra of Finapres and intra-arterial BP. The coherence function is comparable to regression analysis in the time domain, except that a correlation is computed for each frequency region ${ }^{12}$. The coherence function ranges between 0 (no relation) and 1 (a perfect relation). With respect to details of data analysis and transfer function (coherence) we refer to an earlier report of our group ${ }^{13}$. The spectral resolution was $0.016 \mathrm{~Hz}(1 / 64 \mathrm{~s})$, yielding seven (coherence) values for the LF $(0.04-0.15 \mathrm{~Hz})$ band and 70 values for the HF $(0.4-1.5 \mathrm{~Hz})$ band. In each subject, a mean coherence $\pm \mathrm{SD}$ was computed for the LF and $\mathrm{HF}$ band and was used to assess the statistical reliability of the two power spectra. Pooled (group) results of the coherence values are presented as mean \pm standard error of measurements (SEM).

Comparisons between SBP and DBP values or between LF and HF values were made with a (two-sided) paired $t$ test for parametric data. Statistical significance was accepted at $\mathrm{p}<0.05$.

\section{Results}

Reliable Finapres waveform signals could be obtained in eight (gestational age, range, 26.1-33.4 wk; weight, range, 1000-2280 g) of 12 subjects. All failures were due to bad fitting of the cuff around the small wrist in infants with a weight $<1000 \mathrm{~g}$. All but one infant were circulatory stable at the time of measurement, and echocardiography in these subjects revealed no structural abnormalities. One infant (subject 7) with a patent ductus arteriosus required vasoactive drugs to maintain adequate BP.

A total of 2502 paired BP measurements were analyzed, yielding a mean of 313 analyzed beats per selected 128-s period per subject (range, 300-345). A representative tracing of beat-to-beat SBP values obtained in subject number 1 is shown in Figure 1. The mean intra-arterial BP was $55.6 \pm 10.2 \mathrm{mmHg}$ systolic and $30.0 \pm 5.5 \mathrm{mmHg}$ diastolic in the study group. Table 1 shows the individual results of comparing absolute BP values. We observed considerable variation in under- and overestimation of the intra-arterial pressure values between subjects. Bias ranged from -6.5 to $+17.6 \mathrm{mmHg}$ systolic and from -5.6 to $+17.9 \mathrm{mmHg}$ diastolic. Details of the individual $95 \%$ limits of agreement are shown in Table 1. The mean correlation coefficients were $0.78 \pm 0.18$ systolic and $0.80 \pm 0.11$ diastolic (NS). The mean gains of absolute BP measurements were $1.0 \pm 0.3 \mathrm{mmHg} / \mathrm{mmHg}$ systolic and $0.8 \pm 0.2 \mathrm{mmHg} / \mathrm{mmHg}$ diastolic (NS), with 


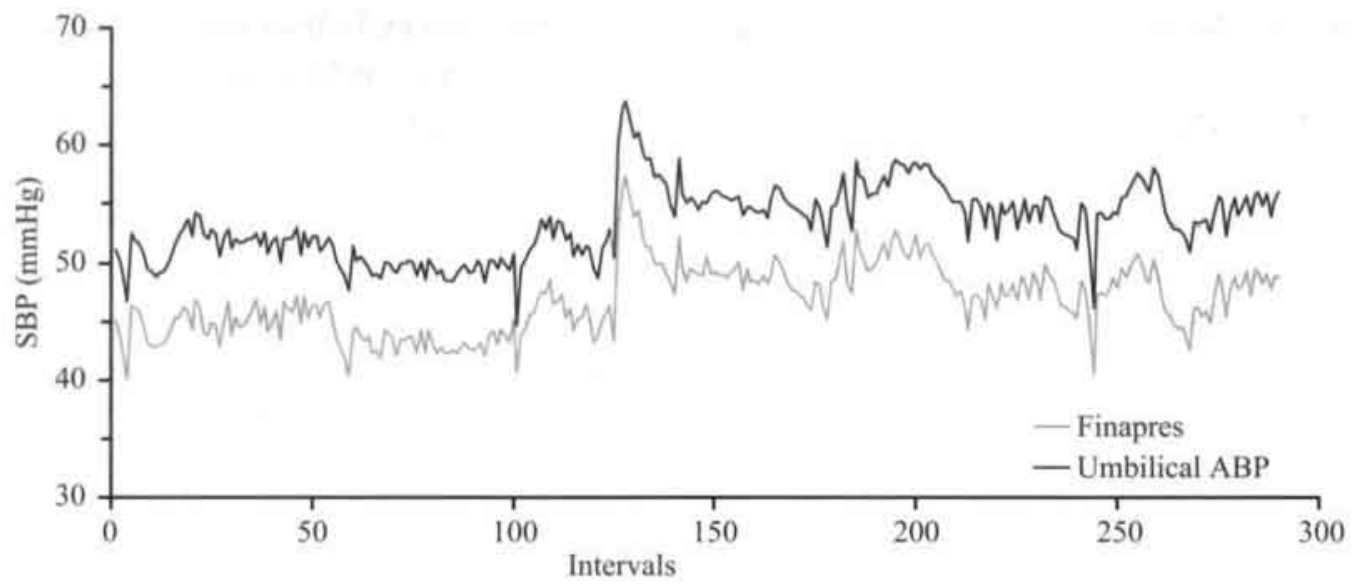

Figure 1. Tracing of SBP values obtained in a preterm infant of $1260 \mathrm{~g}$ (subject 1 ). This tracing shows beatto-beat intra-arterial (black line) and Finapres (grey line) SBP values of a selected period of $128 \mathrm{~s}$. Note the difference (bias) in SBP values (approximately $6.5 \mathrm{mmHg}$ ) between both methods, but with accurate tracking the $\mathrm{BP}$ changes.

Table 1: Results of absolute BP values obtained by both methods of the study group

\begin{tabular}{lrrrrr} 
Subject nr. & \multicolumn{2}{c}{ bias } & & \multicolumn{2}{c}{$\mathbf{9 5 \%}$ limits of agreement } \\
\cline { 2 - 4 } & $S B P$ & $D B P$ & & $S B P$ & $D B P$ \\
\hline 1 & -6.5 & -3.5 & -8.1 to -5.0 & -5.7 to -1.3 \\
2 & -5.7 & 1.7 & -7.3 to -4.1 & 0.9 to 2.5 \\
3 & -4.5 & 2.1 & -6.3 to -2.7 & 0.3 to 3.9 \\
4 & 4.4 & 17.9 & -1.4 to 10.2 & 14.7 to 21.1 \\
5 & 7.0 & 4.9 & 3.4 to 10.6 & 2.7 to 7.1 \\
6 & 5.2 & 7.9 & 3.4 to 7.0 & 6.7 to 9.1 \\
7 & 17.6 & -5.6 & 13.6 to 21.6 & -9.4 to -1.8 \\
8 & 0.3 & -4.5 & -1.9 to 2.5 & -5.7 to -3.3
\end{tabular}

Bias (mean of the difference) and 95\% limits of agreement for SBP and DBP, respectively. Bias and $95 \%$ limits of agreement are expressed in $\mathrm{mmHg}$. 
offsets of $+3.2 \pm 12.9$ and $+7.7 \pm 6.9 \mathrm{mmHg}$, respectively (NS).

In Table 2 the beat-to-beat changes of Finapres and arterial BP signals are presented. Remarkably, no bias existed between both methods for the beat-to-beat BP values. Details of the individual $95 \%$ limits of agreement are shown in Table 2. The mean correlation coefficients were $0.87 \pm 0.11$ systolic and $0.76 \pm 0.22$ diastolic $(\mathrm{p}=0.05)$. The mean gains of beat-to-beat BP measurements were $1.0 \pm 0.4 \mathrm{mmHg} / \mathrm{mmHg}$ systolic and $0.8 \pm 0.2$ diastolic (p value $<0.05$ ), with offsets of $0.0 \pm 0.0$ and $0.0 \pm 0.0 \mathrm{mmHg}$, respectively (NS). The diastolic beat-to-beat gain $(<1)$ indicates increasing negative bias with increasing beat-to-beat BP changes.

Table 2: Results of beat-to-beat changes between arterial BP and Finapres signals

\begin{tabular}{|c|c|c|c|c|}
\hline \multirow[t]{2}{*}{ Subject nr. } & \multicolumn{2}{|c|}{ bias } & \multicolumn{2}{|c|}{$95 \%$ limits of agreement } \\
\hline & SBP & $D B P$ & $S B P$ & $D B P$ \\
\hline 1 & 0.0 & 0.0 & -1.2 to 1.2 & -1.4 to 1.4 \\
\hline 2 & 0.0 & 0.0 & -0.8 to 0.8 & -0.7 to 0.7 \\
\hline 3 & 0.0 & 0.0 & -2.1 to 2.1 & -2.0 to 2.0 \\
\hline 4 & 0.0 & 0.0 & -3.1 to 3.1 & -1.9 to 1.9 \\
\hline 5 & 0.0 & 0.0 & -2.1 to 2.1 & -1.9 to 1.9 \\
\hline 6 & 0.0 & 0.0 & -1.7 to 1.7 & -1.1 to 1.1 \\
\hline 7 & 0.0 & 0.0 & -3.3 to 3.3 & -2.2 to 2.2 \\
\hline 8 & 0.0 & 0.0 & -1.7 to 1.7 & -1.4 to 1.4 \\
\hline
\end{tabular}

Bias (mean of the difference) and 95\% limits of agreement for SBP and DBP beat-to-beat changes, respectively. Bias and $95 \%$ limits of agreement are expressed in $\mathrm{mmHg}$.

Figure 2 illustrates the power spectra of intra-arterial and Finapres systolic signals in subject 1 . Note that the two spectra look similar. In this subject, the mean coherence value for $\mathrm{LF}$ was $0.97 \pm 0.03$ and for HF $0.94 \pm 0.06$. In Table 3, the mean coherence values for the $\mathrm{LF}$ and $\mathrm{HF}$ bands are shown for the total group. For LF fluctuations, the (pooled) group mean \pm SEM coherence values were $0.93 \pm 0.00$ systolic and $0.91 \pm 0.01$ diastolic (NS). For HF fluctuations, the mean coherence values were $0.91 \pm 0.01$ systolic and $0.82 \pm 0.02$ diastolic ( $\mathrm{p}=0.07$, NS). For SBP, no significant difference was found in coherence values between LF and HF. For DBP, a significant difference in coherence values was found between LF and HF ( $p<0.05$ ). 


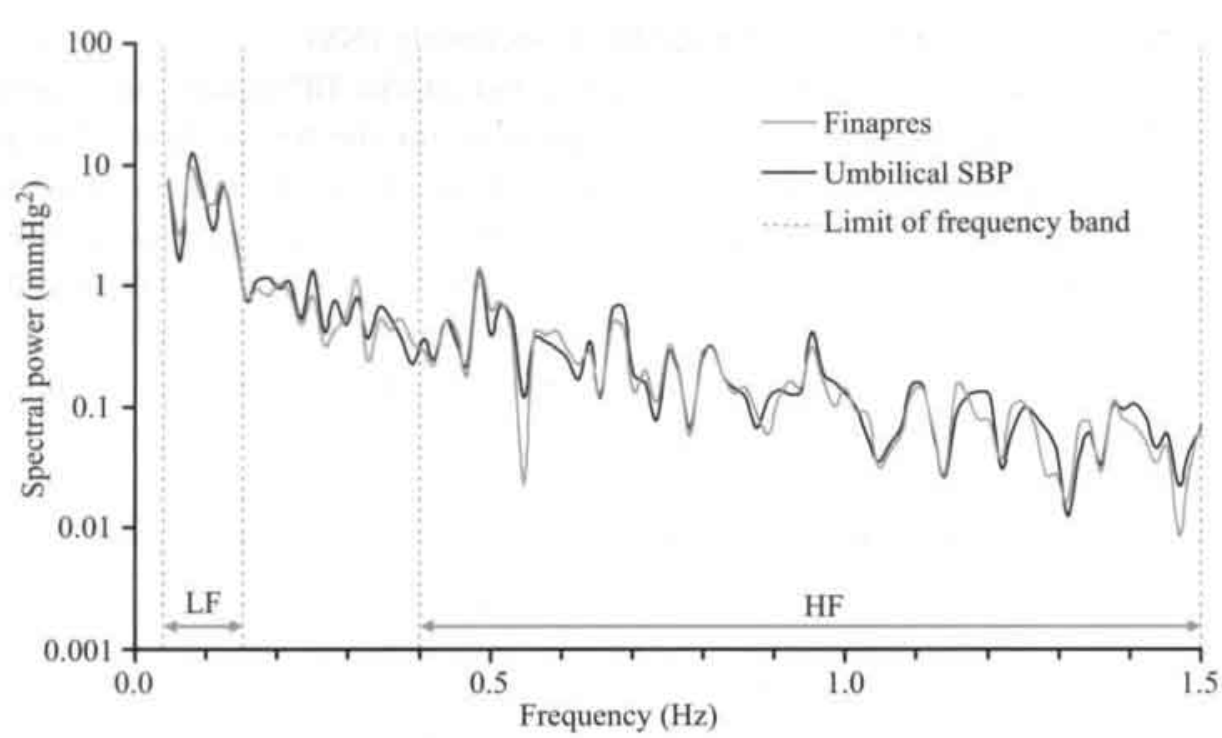

Figure 2. Spectral power curves obtained by both methods of the same infant as in Figure 1, In this figure, two power spectra of intra-arterial systolic blood pressure (black line) and Finapres (grey line) measurements are shown of the SBP data. The LF band was defined between 0.04 and $0.15 \mathrm{~Hz}$. The HF band was defined between 0.4 and $1.5 \mathrm{~Hz}$. Note that the two power spectra look similar. Spectral power (logarithmic scale) was expressed in $\mathrm{mmHg}^{2}$. In this subject the mean coherence value for $\mathrm{LF}$ was $0.97 \pm$ 0.03 and for HF $0.94 \pm 0.06$.

\section{Discussion}

We found considerable differences between the noninvasive Finapres technique and umbilical arterial BP measurements for absolute BP values, with a great variation in bias and limits of agreement in the study group. In contrast, we found a good correlation between beat-to-beat values and no bias between both methods, indicating accurate estimation of beat-to-beat changes of BP values. In addition, we found high coherence values between noninvasive and intra-arterial BP power spectra in both HF and LF bands.

The only available study in neonates, using the same wrist position, reported comparable results with the $95 \%$ limits of agreement -5.3 to $+8.7 \mathrm{mmHg}$ for SBP and -5.2 to $+5.5 \mathrm{mmHg}$ for DBP5 . Their conclusion of "both methods gave similar results" needs further clarification, in our opinion. In contrast to our method of analyzing a complete 2-min period, they analyzed five very short periods of $5 \mathrm{~s}$ resulting in a limited amount of BP values. From Table 1, it is clear that there is considerable variation in bias among the subjects. It is therefore questionable whether one may pool all individual BP values, as done in the former study. In addition, we can expect more 
Table 3: The coherence values of the LF $(0.04-0.15 \mathrm{~Hz})$ and HF $(0.4-1.5 \mathrm{~Hz})$ bands for SBP and DBP

\begin{tabular}{|c|c|c|c|c|}
\hline \multirow[t]{2}{*}{ Subject nr. } & \multicolumn{2}{|c|}{ LF band } & \multicolumn{2}{|c|}{ HF band } \\
\hline & SBP & DBP & SBP & DBP \\
\hline 1 & $0.97 \pm 0.03$ & $0.89 \pm 0.16$ & $0.94 \pm 0.06$ & $0.85 \pm 0.13$ \\
\hline 2 & $0.95 \pm 0.03$ & $0.93 \pm 0.05$ & $0.92 \pm 0.08$ & $0.80 \pm 0.17$ \\
\hline 3 & $0.99 \pm 0.01$ & $0.94 \pm 0.04$ & $0.95 \pm 0.05$ & $0.93 \pm 0.12$ \\
\hline 4 & $0.93 \pm 0.05$ & $0.96 \pm 0.04$ & $0.93 \pm 0.08$ & $0.87 \pm 0.14$ \\
\hline 5 & $0.91 \pm 0.10$ & $0.87+0.15$ & $0.91 \pm 0.07$ & $0.60 \pm 0.19$ \\
\hline 6 & $0.97 \pm 0.02$ & $0.98 \pm 0.01$ & $0.94 \pm 0.05$ & $0.96 \pm 0.05$ \\
\hline 7 & $0.90 \pm 0.08$ & $0.83 \pm 0.08$ & $0.78 \pm 0.15$ & $0.83 \pm 0.16$ \\
\hline 8 & $0.84 \pm 0.11$ & $0.90 \pm 0.08$ & $0.94 \pm 0.07$ & $0.74 \pm 0.17$ \\
\hline group mean $\pm S E M$ & $0.93 \pm 0.00$ & $0.91 \pm 0.01$ & $0.91 \pm 0.01$ & $0.82 \pm 0.02^{*}$ \\
\hline
\end{tabular}


pronounced differences between both methods in our population than in adults, because we compare lower aortic pressure with "radial" BP. More distal in the arterial tree, the BP waveform generally shows overshoot of the SBP. Because this overshoot depends on characteristics of the arterial tree, which may vary substantially between subjects, Finapres is not reliable in estimating absolute BP values. Like others, we observed substantial variation in under- and overestimation of the intra-arterial pressure values between subjects ${ }^{11.14}$. Vasoconstriction in relation to temperature seems unlikely because the incubator preserves a thermoneutral environment. In addition, variation might be related to cuff fitting due to the small size of the wrist, or tissue edema. The used physiocal algorithm for servo calibration was not developed for cuff application at the wrist. Therefore, the algorithm does not always succeed in finding the correct cuff pressure for vascular unloading. The averaged SD of the difference between both methods was low for SBP $(1.4 \pm 0.8)$ and DBP $(1.0 \pm 0.5)$, indicating consistency of measurement for individual subjects. In four subjects with birth weight $<1000 \mathrm{~g}$ no reliable signals could be obtained because of the very small size of the wrist.

In pediatric patients, it is concluded that although substantial measurement bias exists between the noninvasive technique and intra-arterial BP measurements, frequency response analysis suggests that noninvasive BP measurement accurately follows BP changes" . Also, we found a very good correlation between beat-to-beat values between both methods indicating accurate estimation of beat-to-beat changes of particularly SBP values. The SD of successive beat-to-beat changes in heart rate or BP within a certain period provides a rough estimate of short-term variability. The (time-domain) shortterm variability is similar to the (frequency-domain) HF spectral peak, associated with respiratory activity ${ }^{9}$. For systolic beat-to-beat measurements, we observed a higher correlation than for diastolic values. The gain close to 1 and offset of 0 for systolic beatto-beat values indicates no systematic error in over- or underestimation of intra-arterial systolic measurements. The gain for diastolic (beat-to-beat) values (mean approximately 0.8 ), however, indicates increasing negative bias with increasing beat-tobeat BP changes. These findings suggest that Finapres estimate systolic changes more accurately than diastolic changes.

In addition, we found very high coherence values between arterial and Finapres BP power spectra in both HF and LF bands. This strongly indicates a high amount of linear coupling between the two power spectra of Finapres and intra-arterial BP. No differences were found between LF and HF bands for systolic values, indicating that no overestimation was present for the LF fluctuations as seen in adult ${ }^{6}$. Although it did not reach statistically significance, we observed a tendency for a higher amount of linear coupling in the HF band for SBP than for DBP measurements. 


\section{Conclusion}

In conclusion, this study demonstrates that Finapres estimates SBP changes accurately and estimates BP power spectra reliably. Because baroreflex sensitivity specifies the ratio between changes in RR interval time and changes in SBP $(\mathrm{ms} / \mathrm{mmHg})$, Finapres could be useful in clinical research to quantify this measure. Spontaneous baroreflex sensitivity has been assessed using (the wrist method) Finapres in neonates by time domain analysis ${ }^{15}$. In addition, this noninvasive technique enables use of cross-spectral analysis (transfer function analysis) of heart rate and BP fluctuations ${ }^{13}$. Finapres thus provides a noninvasive tool for investigating the autonomic cardiovascular regulation in neonates. 


\section{References}

1. Penaz J, Voigt A, Teichmann W 1976 Beitrag zur fortlaufenden indirekten Blutdruckmessung. Z Gesamte Inn Med 31:1030-3

2. Wesseling KH, Settels JJ, de Wit B 1986 The measurement of continuous finger arterial pressure noninvasively in stationary subjects. In: Schmidt TH, Dembroski TM. Bluemchen G (eds) Biological and Psychological Factors in Cardiovascular disease. Berlin, Germany: Springer-Verlag, pp 355-375

3. Parati G, Casadei R, Groppelli A, di Rienzo M, Mancia G 1989 Comparison of finger and intraarterial blood pressure monitoring at rest and during laboratory testing. Hypertension 13:647-655

4. Tanaka H, Thulesius O, Yamaguchi, Mino M, Konishi K 1994 Continuous non-invasive finger blood pressure monitoring in children. Acta Paediatr 83(7):646-652

5. Drouin E, Gournay V, Calamel J. Mouzard A. Rozé JC 1997 Feasibility of using finger arterial pressure in neonates. Arch Dis Child Fetal Neonatal Ed 77:F139-140

6. Omboni S. Parati G, Frattola A, Mutti E, Di Rienzo M, Castiglioni P. Mancia G 1993 Spectral and sequence analysis of finger blood pressure variability. Comparison with analysis of intra-arterial recordings. Hypertension 22:26-33

7. Jaffe RS, Fung DL 1994 Constructing a heart rate variability analysis system. J Clin Monit 10:45-58

8. Akselrod S 1995 Components of heart rate variability: basic studies. In: Malik M, Camm AJ (eds.) Heart rate variability. Futura Publishing Company, New York, pp 147-163

9. Rosenstock EG, Cassuto Y. Zmora E 1999 Heart rate variability in the neonate and infant: analytical methods, physiological and clinical observations. Acta Paediatr 88:477-82

10. Bland JM. Altman DG 1986 Statistical methods for assessing agreement between two methods of clinical measurement. Lancet I(8476):307-310

11. Triedman JK. Saul JP 1994 Comparison of intra arterial with continuous non-invasive blood pressure measurements in postoperative pediatric patients. J Clin Monit 10:11-20

12. de Boer RW, Karemaker JM, Strackee J 1985 Relationships between short-term blood pressure fluctuations and heart rate variability in resting subjects: a spectral approach. Med Biol Eng Comput 23:352-358

13. Andriessen P. Koolen AMP. Berendsen RCM. Wijn PFF, ten Broeke EDM, Oei SG, Blanco CE 2003 Cardiovascular fluctuations and transfer function analysis in stable preterm infants. Pediatr Res 53:89-97

14. Jones RDM, Brown AG, Roulson CJ, Smith ID, Chan SC 1992 The upgraded Finapres 2300e: a clinical evaluation of a continuous noninvasive blood pressure monitor. Anaesthesia 47:701-705

15 Drouin E, Gournay V, Calamel J, Mouzard A, Rozé JC 1997 Assessment of spontaneous baroreflex sensitivity in neonates. Arch Dis Child Fetal Neonatal Ed 76:F108-112 


\section{Chapter 6}

\section{Cardiovascular fluctuations and transfer function analysis in stable preterm infants}

Peter Andriessen, André M.P. Koolen, Ralph C.M. Berendsen, Pieter F.F. Wijn, Edith D.M. ten Broeke, S. Guid Oei, Carlos E. Blanco

Pediatric Research 2003;53:89-97

www.pedresearch.org/content/vol53/issue1/ 


\section{Abstract}

To examine the baroreceptor reflex function, a beat-to-beat analysis between systolic blood pressure (SBP) and R-R interval fluctuations was studied in 10 stable appropriate-for-gestational age preterm infants (range, 27.2-33.7 wk) in the first postnatal week during quiet sleep. Spectral power analysis, using fast Fourier transform, and transfer functions (gain and phase difference) between SBP and R-R fluctuations were estimated in a low frequency band (LF, 0.03-0.2 Hz) and high frequency band (HF defined as the frequency band between the 10 and 90 -centile of the individual respiratory frequency). The LF/HF ratio reflects the sympathovagal balance. The mean frequency $( \pm$ SD) of LF peaks was centered at $0.07 \pm 0.02 \mathrm{~Hz}$. The mean frequency $( \pm \mathrm{SD}$ ) of the individual $\mathrm{HF}$ band was $0.82 \pm 0.21 \mathrm{~Hz}$. The LF/HF ratio in R$\mathrm{R}$ interval series (median, 29; interquartile range (IQR), 16-40) was higher than in SBP series (median, 8; IQR, 4-14). The gain between R-R interval and SBP fluctuations (median, $4.2 \mathrm{~ms} / \mathrm{mmHg}$; IQR, 2.4-5.0) in the LF band was higher than in the HF band (median, $1.7 \mathrm{~ms} / \mathrm{mmHg}$; IQR, 1.4-3.0). SBP fluctuations lead R-R interval fluctuations in the LF band with a median phase difference of $+96^{\circ}$ (IQR, 67-132). At LF the fluctuations in SBP precede changes in R-R interval with a time delay of $3.8 \mathrm{~s}$.

These observations indicate a dominant role of the sympathetic system in stable preterm infants in comparison with published adult values. Cross-spectral analysis allows a test for tracking the development of the sympathetic system in neonates.

\section{Abbreviations}

BP blood pressure

BR baroreceptor reflex

BW birth weight

GA gestational age

HF high frequency (frequency between p-10 and p-90 of the individual respiratory frequency)

HR heart rate

IQR interquartile range (P25 - P75)

LF low frequency $(0.03-0.2 \mathrm{~Hz})$

PCA postconceptional age

$\mathrm{R}-\mathrm{R} \quad \mathrm{R}-\mathrm{R}$ interval length

SBP systolic blood pressure 


\section{Introduction}

The autonomic nervous system plays a key role in blood pressure (BP) control by adapting heart rate (HR) and total peripheral resistance. Multiple physiological mechanisms are responsible for fluctuations in HR and BP: respiration, baroreceptor reflex (BR), and thermoregulation. Power spectral analysis of the R-R interval series permits us to evaluate aspects of cardiovascular autonomic control ${ }^{1}$. A high frequency (HF) spectral peak occurs at the respiratory frequency. A low frequency (LF) spectral peak, synchronous with Mayer waves in BP, occurs around $0.1 \mathrm{~Hz}$ and is attributed to the $\mathrm{BR}^{2}$. Very low frequency oscillations (below $0.03 \mathrm{~Hz}$ ) are ascribed to peripheral resistance fluctuations caused by thermoregulation. HF fluctuations are associated with the parasympathetic system, whereas LF fluctuations are related to sympathetic and parasympathetic activity ${ }^{3}$. The $\mathrm{LF} / \mathrm{HF}$ ratio reflects the sympathovagal balance ${ }^{3}$.

The BR can be considered a closed loop circuit: BP fluctuations affect R-R interval fluctuations and visa versa ${ }^{4}$. Spectral analysis technique and BR activation through postural change enable the assessment of BR gain/sensitivity in human adults 5.6 . Cardiovascular reflexes using spectral power analysis of $\mathrm{R}-\mathrm{R}$ interval series have been studied in preterm and full-term infants ${ }^{7-11}$. The LF/HF ratio progressively decreases with postnatal age, indicating an increase in parasympathetic contribution to control HR ${ }^{8,9.11}$. In general, these findings suggest that in preterm infants BR becomes more functional with postnatal development. To our knowledge cross-spectral analysis of BP and $\mathrm{R}-\mathrm{R}$ interval fluctuations have not been examined earlier in preterm and term infants for the estimation of BR sensitivity. This stimulated our interest in evaluating the cardiovascular autonomic control in stable preterm infants by studying the relationship between spontaneous fluctuations of invasively measured BP and R-R interval signals.

The objectives of this investigation were to determine the feasibility of cross-spectral analysis of R-R interval and systolic BP (SBP) series, evaluating the sympathovagal balance (LF/HF ratio) in both R-R intervals series and SBP series, and assessing the transfer function (BR gain/sensitivity and phase) in stable preterm infants.

\section{Methods}

Subjects: The study group consisted of 10 stable preterm infants. The gestational age (GA) ranged from 27.2 to $33.7 \mathrm{wk}$ and birth weight (BW) ranged from 800 to $2050 \mathrm{~g}$. Six infants were born spontaneously, and four were delivered by caesarean section. All infants received prenatal steroids. The 5-min Apgar ranged between 5 and 10, and the 
umbilical arterial blood pH ranged between 7.2 and 7.4. All infants were appropriatefor-gestational age, according to the Dutch growth charts ${ }^{12}$. All infants were studied once in the immediate post-intensive care phase between 2 to 8 days of postnatal life. Arterial catheters were neither inserted nor remained longer in place because of the study. The postconceptional age (PCA) ranged from 28.1 to $34.3 \mathrm{wk}$. All infants were breathing room air spontaneously. There were no signs or symptoms of asphyxia, respiratory distress, sepsis, or patency of the arterial duct at the time of the study. None had echoencephalographic evidence of cerebral hemorrhage or parenchymal lesions. Echocardiography revealed no structural abnormalities. All subjects received caffeine therapy (blood levels, $11-17 \mathrm{mg} / \mathrm{l}$ ) to prevent apnea. Caffeine was administered once a day at midnight. Recordings were made at least 12 hours after caffeine administration. Cardiotropic drugs were not used. Values of electrolytes, blood gas analysis, hematocrits were all within normal range. Informed consent was obtained from the parents of each infant. The study was approved by the local Ethics Committee.

Data acquisition: A bipolar chest lead of the surface ECG and the transthoracic electric impedance waveforms were recorded by Hewlett Packard neonatal monitor type Merlin (Waltham, MA, USA). Arterial BP was measured invasively through a 4 French catheter placed earlier in the lower aortic position for routine monitoring of vital functions and intensive care management. A $0.5 \mathrm{ml} / \mathrm{h}$ infusion of heparinized physiological saline solution was continuously flushed through the catheter. Recordings were made in the prone position for 1-2 hours. Data segments were selected subsequently during periods of regular breathing and spontaneous sleep with closed eyes and without gross body movements (quiet sleep state) ${ }^{13}$. Data analysis was performed on 192-s-long stationary segments. Because preterm infants have an immature and irregular respiratory drive, 3 -min segments were chosen as a compromise between the demands of sufficient duration and signal stability. The quality of the signals was visually verified for artifacts, ectopic beats, baseline drifts, apnea periods, or gross bradycardia.

Frequency ranges of interest: The whole frequency band of interest was the range between 0.03 and $2 \mathrm{~Hz}$. The very low frequency band $(0-0.03 \mathrm{~Hz})$ was discarded to avoid possible contribution of slow trend artifacts. In addition, fast Fourier transform has a limited accuracy of power estimation in this frequency range using records of relatively short duration. The LF band was defined as the frequency range between 0.03 and $0.2 \mathrm{~Hz}$. Because the HF band primarily contains the reflection of respiratory activity, the HF band was individualized for each subject depending on his or her respiratory drive. The respiration rate was estimated by peak detection of the thoracic 
waveforms resulting in a mean value and a bandwidth between the $10^{\text {th }}$ and $90^{\text {th }}$ centile (p-10 and p-90, respectively) of the breath-by-breath frequency. The frequency range between these centile values was used to identify each subject's individual HF band. The upper spectral limit of the HF band (the $90^{\text {th }}$ centile of the respiratory frequency) was less than half of the mean HR, thereby meeting the requirements of the Nyquist critical frequency. An example of a spectral power density curve is shown in Figure 1.

Data analysis: The sample frequency was $400 \mathrm{~Hz}$ for the ECG signals and $100 \mathrm{~Hz}$ for the arterial BP and transthoracic electric impedance signals. The sampling error of R top detection was optimized using a second-order polynomial fit, resulting in an accuracy of R top detection of $0.2 \mathrm{~ms}$. R waves were detected from the ECG, and an unevenly spaced R-R interval sequence was created. This sequence was resampled using a boxcar window at $4 \mathrm{~Hz}$ to obtain an equidistantly spaced time series ${ }^{14}$. The mean $\mathrm{R}-\mathrm{R}$ interval was calculated and subtracted from all data points to remove the direct current component. SBP was identified from peak detection of the BP signal, resulting in an unevenly spaced "systogram". The "systogram" was converted into an equidistantly spaced time series using the same resampling method as used for the R-R interval time series. Each 192-s-long segment of preprocessed evenly spaced R-R interval and SBP series was subdivided in 5 half-overlapping 64 -s ( 256 points) segments. To reduce the effects of spectral leakage each segment was multiplied with a triangular Parzen window $^{15}$. Fast Fourier transform was used to compute the auto- and cross-spectral density functions for each segment. The spectral resolution was $0.016 \mathrm{~Hz}$. Filter correction removed unwanted side effects of the earlier resampling and Parzen windowing. A mean discrete amplitude spectrum was derived from the 5 autospectral density functions. Powers in the ranges of interest were calculated from the obtained mean autospectral density functions. The total power was computed for the frequency band between 0.03 and $2 \mathrm{~Hz}$. The values for power are presented in units of square milliseconds (for R-R interval series) and square millimeters of mercury (for SBP series). The LF and HF were expressed as normalized units [e.g. LF/total power $x$ $100 \%]^{3}$. Using 64-s segments (resulting in a spectral resolution of $0.016 \mathrm{~Hz}$ ) and 5 halfoverlapping segments power estimation above $0.03 \mathrm{~Hz}$ is accurate. R-R interval and SBP series can be considered as a combination of noise-like and signal-like data with a Gaussian distribution. The relative error of the value per frequency bin is $100 \%$ of the calculated power spectral density 16,17 . Averaging five spectra leads to a relative error of mean spectral power of $23 \%$ per frequency bin.

Transfer function analysis: The amount of linear coupling between two signals in the frequency domain can be expressed by means of the (squared) coherence 

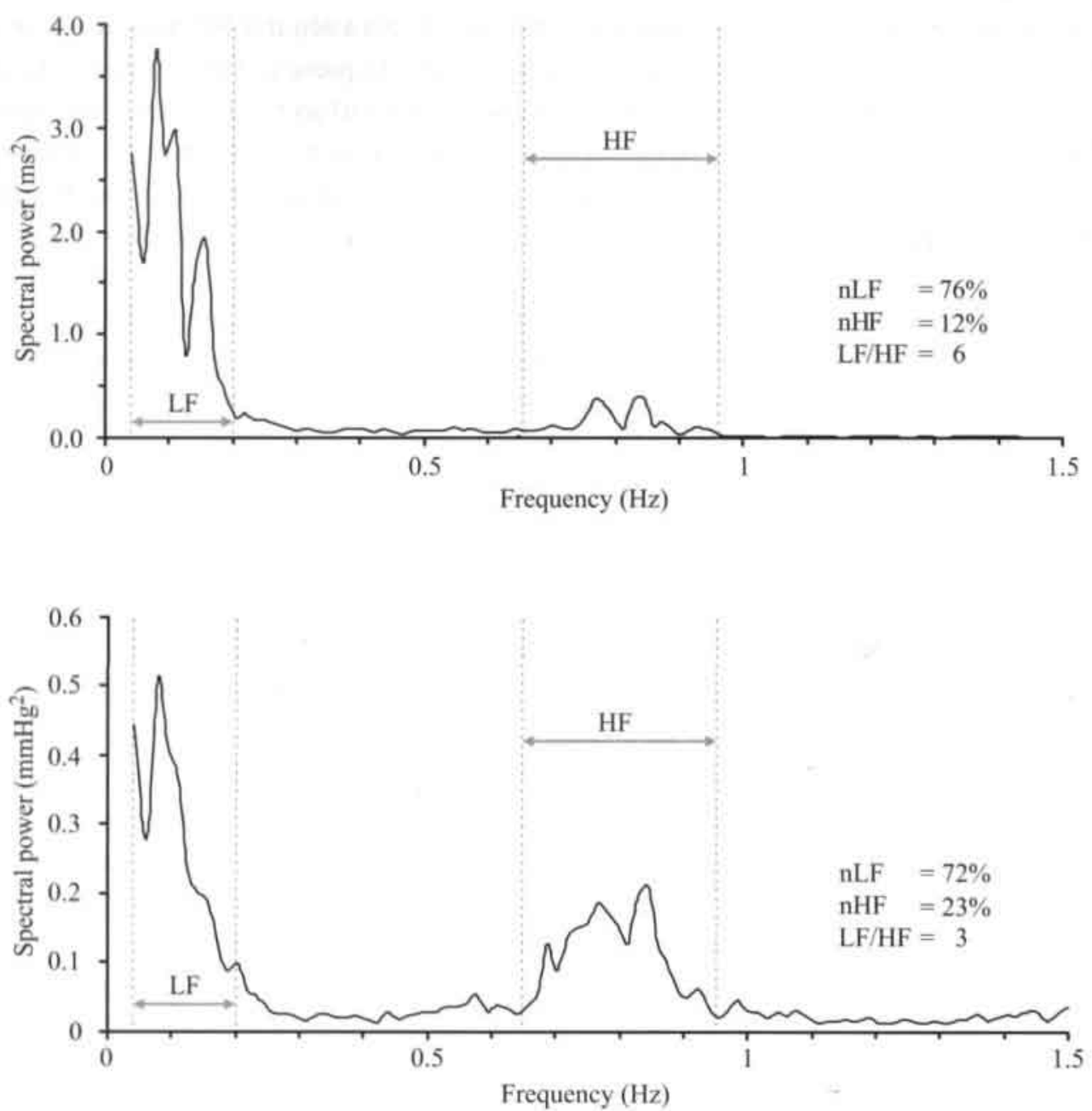

Figure 1. Spectral power curves of R-R interval and SBP series of a spontaneously breathing infant at a PCA of 30 wk. Top, spectral power curve of R-R interval series. The LF band was defined between 0.03 and $0.2 \mathrm{~Hz}$ and is marked between the vertical lines. A clear LF peak was centered at $0.08 \mathrm{~Hz}$. The individual $\mathrm{HF}$ band was defined between the p- 10 and $\mathrm{p}-90$ value of the respiratory frequency and is marked between the vertical lines. A modest HF peak was determined at approximately $0.8 \mathrm{~Hz}$ and ranged between $0.65(\mathrm{p}-10)$ and $0.95(\mathrm{p}-90) \mathrm{Hz}$. LF and HF are expressed as normalized values $(\mathrm{nLF}=\mathrm{LF} /$ total power $\times 100 \%$ and $\mathrm{nHF}=\mathrm{HF} /$ total power $\times 100 \%$ ) and $\mathrm{LF} / \mathrm{HF}$ value (unitless).

Bottom, spectral power curve of SBP series. The LF peak for SBP was centered at $0.08 \mathrm{~Hz}$. Note the much more pronounced peak in the HF band, resulting in a lower LF/HF value. 
function ${ }^{18}$. This measure is comparable to the regression analysis in the time domain, except that it is computed for each frequency region. The coherence function, which ranges between 0 (no relationship) and 1 (linear relationship), was used to assess the statistical reliability of the transfer function estimates at each frequency. Only data in which the coherence was above 0.5 were used in the estimation of the transfer functions. The transfer functions (gain and phase) between SBP and R-R interval signals were estimated from the average auto- and cross-spectral density functions. Gain and phase were assessed in the LF and HF band at the frequency of highest coherence. The gain (milliseconds per millimeter of mercury) reflects the degree to which the input signal (BP) amplitude becomes manifest in the output signal (R-R interval) amplitude at a discrete frequency. The phase difference (degrees) indicates the lead or lag of one signal with respect to the other at a discrete frequency (Figures 2 and 3).

Spontaneous BR gain/sensitivity: The transfer function gain was used to estimate frequency-specific BR sensitivity during normal physiological fluctuations ${ }^{19-21}$. In addition a time domain estimate was used to describe BR sensitivity. The SD of (R-R)i intervals (square root of variance or root-mean-square for the mean) and SBP beat-tobeat values were determined in the 192-s-long segments. As variance is related to the total power of spectral analysis, the ratio of SD of (R-R)i intervals divided by the SD of SBP (milliseconds per millimeter of mercury) was used as a time domain estimate for total variability gain 22 .

Statistical analysis: Data with a normal distribution are expressed as a mean \pm SD, otherwise data are expressed as a median and IQR. Comparisons were made with (twosided) paired $t$ test for parametric data and the Wilcoxon sign rank test for nonparametric data. The influence of GA, PCA and BW on spectral power values and transfer function parameters was studied by linear regression analysis, using the method of least squares, and ANOVA. Statistical significance was accepted at $\mathrm{p}<0.05$.

\section{Results}

The clinical characteristics of the study population are shown in Table 1. From the 192s-long segments of each infant the mean and SD of the R-R interval, SBP and diastolic BP were calculated.

A representative example of R-R interval spectral power and SBP spectral power of a spontaneously regular breathing preterm infant (mean respiratory frequency, $0.8 \mathrm{~Hz}$; 
Table 1: Clinical characteristics and time domain measures of the study population

Gestational age (wk)

Birthweight (g)

Postnatal age (d)

$\mathrm{R}-\mathrm{R}$ interval (ms)

$\mathrm{SBP}(\mathrm{mmHg})$

$\mathrm{DBP}(\mathrm{mmHg})$

$$
\begin{aligned}
30.2 & \pm 2.1 \\
1369 & \pm 395 \\
5.1 & \pm 1.4 \\
432 & \pm 36 \\
59 & \pm 8 \\
35 & \pm 5
\end{aligned}
$$

Data are expressed as mean \pm SD.

Systolic blood pressure (SBP): diastolic blood pressure (DBP).

Table 2: Spectral power values of the study population

\begin{tabular}{lccc}
\hline & $\begin{array}{l}\text { R-R } \\
\text { interval series }\end{array}$ & SBP series & p value * \\
\hline Total power & $31(28-59)$ & $3.7(2.7-4.8)$ & \\
LF power & $21(18-44)$ & $1.9(1.6-3.5)$ & \\
HF power & $2(1-3)$ & $0.3(0.2-0.6)$ & \\
Normalized LF power $(\%)$ & $74(70-76)$ & $60(50-64)$ & $<0.005$ \\
Normalized HF power $(\%)$ & $3(2-5)$ & $7(4-14)$ & $<0.005$ \\
LF/HF ratio & $29(16-40)$ & $8(4-14)$ & $<0.05$ \\
\hline
\end{tabular}

Spectral powers in the ranges of interest were calculated from the obtained mean autospectral density functions for R-R interval series and systolic blood pressure (SBP) series. The values for spectral power (Total, LF, and HF power) are presented in units of $\mathrm{ms}^{2}$ (for $\mathrm{R}-\mathrm{R}$ interval series) and $\mathrm{mmHg}^{2}$ (for SBP series). In addition the LF and $\mathrm{HF}$ were expressed as normalized values (for example: $\mathrm{LF} /$ total power $\times 100 \%)$. LF/HF values are unitless.

Data are expressed as median and interquartile range. * Wilcoxon sign rank test for non-parametric data.

The total power was computed for the frequency band between 0.03 and $2 \mathrm{~Hz}$. The low frequency (LF) band was defined between $0.03-0.2 \mathrm{~Hz}$. The high frequency (HF) band was defined as the frequency range between the $\mathrm{p}-10$ and $\mathrm{p}-90$ centile of the individual respiratory frequency. 
$\mathrm{P}-10,0.65 \mathrm{~Hz}$ and $\mathrm{P}-90,0.95 \mathrm{~Hz}$ ) at a PCA of $30 \mathrm{wk}$ is shown in Figure 1. In the spectra the following features are characteristic: 1) a higher peak in the LF band compared with the HF band, both in R-R interval and SBP power spectrum; 2) the LF peak is centered around $0.08 \mathrm{~Hz}$, both in R-R interval and SBP power spectrum; and 3) a clear HF peak in the SBP power spectrum and a less pronounced HF peak in the R-R interval power spectrum.

Table 2 displays the resulting spectral power values and the LF/HF ratios for the study group. In all subjects LF and HF peaks could be determined. The mean frequency of the LF peak was $0.07 \pm 0.02 \mathrm{~Hz}$. In all subjects an individual respiratory frequency rate (mean, P-10 and P-90 centile boundaries of the individual HF band) could be calculated. The mean frequency of the individual HF band was $0.82 \pm 0.21 \mathrm{~Hz}$. Normalized LF power in R-R interval series (median, 74\%; IQR, 70-76) was significantly higher than SBP series (median, 60\%; IQR, 50-64). In contrast, the normalized HF power in R-R interval series (median, 3\%; IQR, 2-5) was significantly lower than SBP series (median, 7\%; IQR, 4-14). The LF/HF ratio in R-R interval series (median, 29; IQR, 16-40) was significantly higher than in SBP series (median, 8; IQR, 4-14).

Figures 2 and 3 illustrate examples of the cross-spectral transfer function analysis

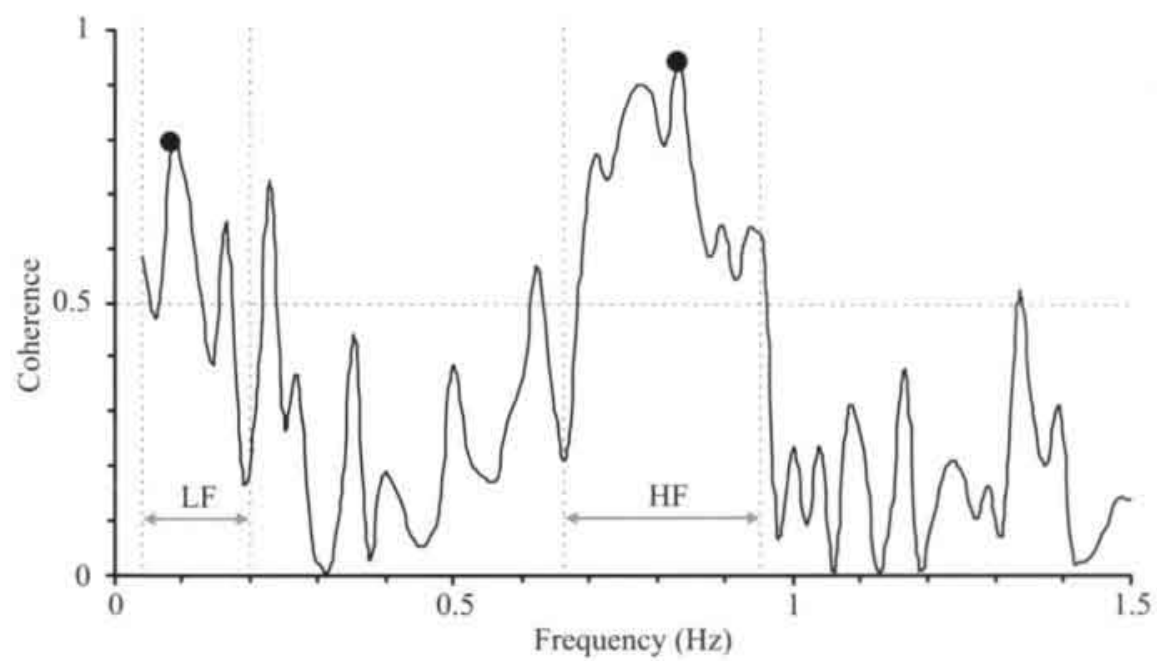

Figure 2. Spectral power curves and coherence of the same infant as in Figure 1. This figure shows the coherence function for the linear relation between SBP and R-R interval series. The vertical lines indicate the boundaries of the LF and HF bands. Note the high coherence in both LF and HF. The horizontal line indicates the coherence value of 0.5 , above this value a reliable estimate was considered for the transfer function parameters. The frequency values with the highest coherence in $\mathrm{LF}(0.08 \mathrm{~Hz})$ and $\mathrm{HF}(0.83 \mathrm{~Hz})$ were chosen (indicated as dots) to compute gain and phase (Figure 3). 

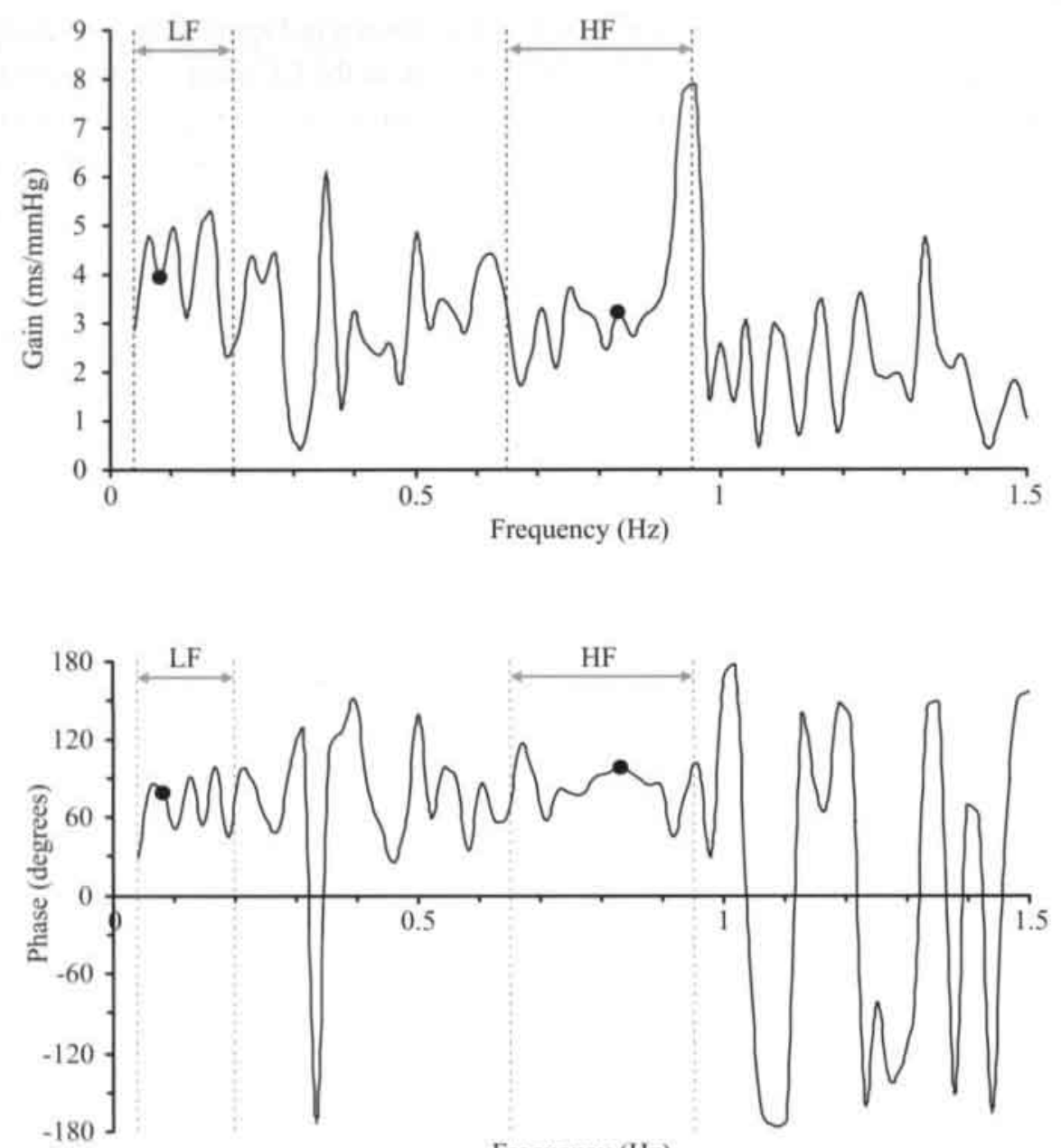

Frequency $(\mathrm{Hz})$

Figure 3. Transfer function parameters of the same infant as in Figure 1. Top, gain as function of frequency. The vertical lines indicate the boundaries of the LF and HF bands. The indicated dots (LF, $3.9 \mathrm{~ms} / \mathrm{mmHg}$; $\mathrm{HF}, 3.2 \mathrm{~ms} / \mathrm{mmHg}$ ) correspond with the frequency values with the highest coherence (see Figure 2).

Bottom, phase as function of frequency. The phase spectrum has positive values if BP fluctuations lead HR fluctuations. The vertical lines indicate the boundaries of the low frequency (LF) and high frequency (HF) bands. The indicated dots (LF, 78 degrees: HF, 97 degrees) correspond with the frequency values with the highest coherence (see Figure 2). 
between R-R interval and SBP signals of the same preterm infant as in Figure 1. The mean and SD of coherence between R-R interval and SBP in the LF band was 0.75 \pm 0.13 , whereas coherence between R-R interval and SBP in the HF band was $0.83 \pm$ 0.09 . The high coherence between R-R interval and SBP implicates reliable estimates of the transfer functions gain and phase. The gain between R-R interval and SBP (median, $4.2 \mathrm{~ms} / \mathrm{mmHg}$; IQR, 2.4-5.0) in the LF band was higher in the HF band (median, $1.7 \mathrm{~ms} / \mathrm{mmHg}$; IQR, 1.4-3.0; $\mathrm{p}<0.05$ ). At LF the phase difference was positive for all subjects; a median phase difference of $+96^{\circ}$ (IQR, 67-132) was calculated. At $\mathrm{HF}$ the phase difference was positive (BP fluctuations leading HR changes; $n=7$ ) or negative (BP fluctuations following HR changes; $n=3$ ), a median phase difference of $+87^{\circ}$ (IQR, -85 to +94 ) was calculated. Mean $R-R$ interval and mean $\mathrm{SBP}$ were not related to any transfer function value.

The median value of the ratio of SD of $(\mathrm{R}-\mathrm{R})_{\mathrm{i}}$ intervals divided by the SD of SBP beatto-beat values was $3.2 \mathrm{~ms} / \mathrm{mmHg}$ (IQR, 2.3-4.5). Figure 4 shows the correlation between LF cross-spectral and time domain gain. LF cross-spectral analysis correlated significantly with the time domain assessment of spontaneous BR sensitivity $\left(r^{2}=0.70\right.$; $y=1.1 x-0.4 ; \mathrm{p}<0.01$ ). HF cross-spectral analysis did not show a significant correlation with the time domain index.

No clear influence of GA, PCA, BW and type of delivery (spontaneous or caesarean section) could be demonstrated on spectral power values, LF/HF ratios and the transfer function parameters.

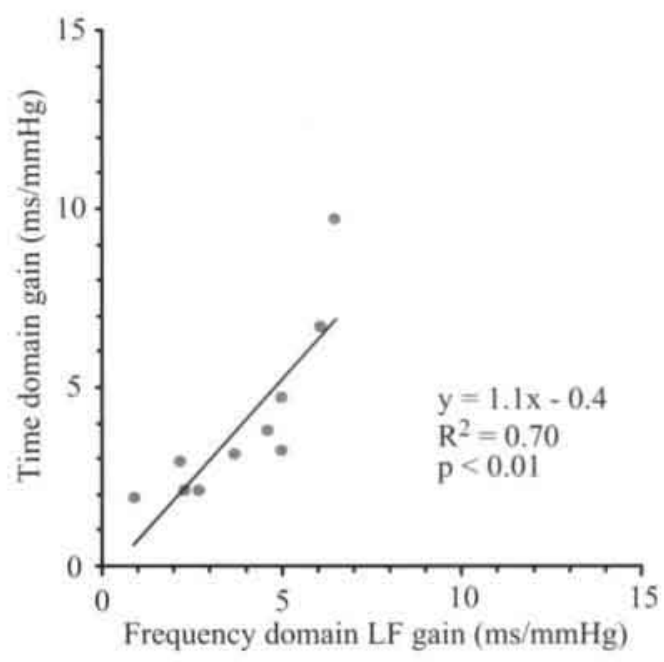

Figure 4. Correlation diagram between cross-spectral LF gain and time domain gain. Correlation between cross-spectral LF gain and time domain estimate for total variability gain ISD of $(R-R)_{i}$ interval series divided by the SD of systolic beat-to-beat blood pressure values]. 


\section{Discussion}

Our data demonstrate for the first time that cross-spectral analysis of R-R interval series and beat-to-beat SBP series is feasible in stable preterm infants. Some of these aspects are different from human adult data $\mathrm{a}^{3-6}$. First, our results show high LF/HF ratios in both $\mathrm{R}-\mathrm{R}$ interval series and beat-to-beat SBP series during resting conditions. Second, the LF peaks of R-R interval and SBP fluctuations are centered around $0.07 \mathrm{~Hz}$. Third, the magnitude of the transfer function (BR gain/sensitivity) between R-R interval and SBP is higher in the LF range (approximately $4 \mathrm{~ms} / \mathrm{mmHg}$ ) than in the HF range (approximately $2 \mathrm{~ms} / \mathrm{mmHg}$ ). In accordance with human adult data ${ }^{4.18}$, we found a consistent positive phase difference between SBP and R-R interval fluctuations in the $\mathrm{LF}$ range. However, our data suggest that preterm infants have a longer time delay of the baroreceptor loop. We found that cross-spectral LF (BR) gain correlates significantly with a time domain estimate for total variability gain.

These observations do not allow us to discuss the participation of the autonomic nervous system in preterm infants in response to abnormal situations (hypotension or hypertension). It would be interesting to know whether more mature preterm infants present a better adaptation capability during pathological conditions. These studies could be difficult to perform as they will be based on challenging the BR loop with different maneuvers to increase or decrease BP. This of course could be pursued using animal models with all their limitations as it is very difficult to work with very premature animal models.

$L F / H F$ ratio: The $\mathrm{LF} / \mathrm{HF}$ ratio in human adults is close to 1 during resting supine condition $^{3}$. We found, both in R-R interval and SBP series, much higher LF/HF ratios. In addition, the higher LF/HF ratio in R-R interval series compared with SBP series was striking. In R-R interval series normalized values of LF power were higher than in SBP series, whereas normalized values of HF power were lower in R-R interval series than in SBP series. Despite differences in methodology (for example, definition of frequency bands) our $\mathrm{LF} / \mathrm{HF}$ ratio values are in the same range as that found by others $8,9,11$. The high LF/HF ratio in preterm infants during quiet sleep could be interpreted as a predominance of sympathetic influence or immature parasympathetic activity. GA has been shown to correlate with decreasing LF/HF ratio in R-R interval series with advancing $\mathrm{GA}^{9.11}$. The decreasing ratio could be consistent with an increase of parasympathetic influence. A low parasympathetic influence in preterm infants could lead to a situation in which the relative fast respiratory-induced BP changes cannot be buffered by modulations of the R-R interval. Only the parasympathetic system is fast enough to influence cardiovascular parameters in the frequency range of respiration in 
preterm infants. The consequence is a relatively high level of BP liability in the HF range, resulting in a lower LF/HF ratio in SBP series than in R-R interval series. In fetal lambs breathing movements were associated with strong fluctuations in BP and weaker fluctuations in $\mathrm{HR}^{23}$. In addition it is speculated that early in life changes in $\mathrm{HR}$ do not contribute to a great degree in reflex control of circulation ${ }^{24}$. However, the differences of HF variability of the two signals are much more complex, because in fetuses the lungs are full of liquid and thus fetal breathing movements are not comparable to the breathing movements of neonates. Still these fetal findings are in accordance with our observations.

The LF peak: In all subjects LF peaks could be determined and the mean of LF fluctuations was centered around $0.07 \mathrm{~Hz}$ (cycle length, $14.3 \mathrm{~s}$ ). This value is in good agreement with an earlier study in neonates concerning heart rate variability ${ }^{25}$. In adults the spectral peak in the LF range is around $0.1 \mathrm{~Hz}$, corresponding to the so-called 10-s rhythm ${ }^{3,26}$. We hypothesize that the natural frequency of the baroreceptor control loop in preterm infants is lower than in adults. However, we have to take in mind that entrainment of the baroreceptor loop is not easy in these tiny infants. We are developing research to explore this hypothesis further by challenging the BR through tilt testing by measuring simultaneously HR, beat-to-beat SBP and blood flow. Relatively low conductivity in the immature nervous system could be responsible for a longer time delay in the BR loop, resulting in a lower natural frequency of the control loop.

The magnitude of the transfer function (BR gain/sensitivity): The gain was calculated for frequencies with a coherence greater than 0.5 , indicating a linear dependency between R-R interval and SBP signals. In our study population of preterm infants the cardiovascular variability was particularly present in the LF range. This resulted in a higher gain of the transfer function between R-R interval and SBP series in the LF range, compared with that in the $\mathrm{HF}$ range. In the LF range the gain was approximately $4 \mathrm{~ms} / \mathrm{mmHg}$, and in the HF range approximately $2 \mathrm{~ms} / \mathrm{mmHg}$. Gain calculated for human adults is much higher, approximately $10-20 \mathrm{~ms} / \mathrm{mmHg}^{4-6,27-29}$. However, in contrast to our findings, the HF gain in adults is higher than LF gain ${ }^{4,5,29}$. Assuming a higher vagal activity in adult life during rest one might expect higher gain in the HF range compared with the LF range in adults. Higher LF gain, however, might indicate a sympathetic predominance in preterm infants. From spontaneous, non respiratory related SBP and R-R interval variations, a BR sensitivity of $4 \pm 2 \mathrm{~ms} / \mathrm{mmHg}$ was assessed in preterm infants ${ }^{30}$. In fetal lambs a gain of $4 \mathrm{~ms} / \mathrm{mmHg}$ was assessed using norepinephrine to increase $\mathrm{BP}^{31}$. Data in neonatal rabbits and lambs showed the relationships between $\mathrm{BP}$ and $\mathrm{R}-\mathrm{R}$ interval fluctuations are greatest at lower 
frequencies ${ }^{19,32.33}$. Despite differences in methodology and species, the results of these (neonatal) studies are in accordance with our data and differ from human adult data. To our knowledge no comparative transfer function data are available in full-term infants. The reason for this lack of data is unclear, but may be related to the fact that small preterm infants have arterial lines for monitoring and blood withdrawal, whereas term babies with lines are mostly asphyxiated or in extreme respiratory distress and do not represent normal physiology.

The phase of the transfer function: The phase of the transfer function indicates the temporal relationship between the signals in the frequency domain ${ }^{6.19}$. However, in case of an intact baroreceptor closed loop system, judgment considering causality and interpretation of the phase spectrum is hampered, because it is not clear whether one signal leads another by $360^{\circ}+n$ degrees or is behind by $360^{\circ}-n$ degrees. The phase difference of $+96^{\circ}$ at $0.07 \mathrm{~Hz}$ (cycle length, $14.3 \mathrm{~s}$ ), represents a time delay of $3.8 \mathrm{~s}$. We interpreted this as at LF, changes in BP lead the change in R-R interval for the following reasons. First, consistent with $\mathrm{BR}$ activity is $\mathrm{R}-\mathrm{R}$ interval lengthening in response to an increase in BP, or R-R interval shortening in response to a decrease in $\mathrm{BP}^{30}$. Second,

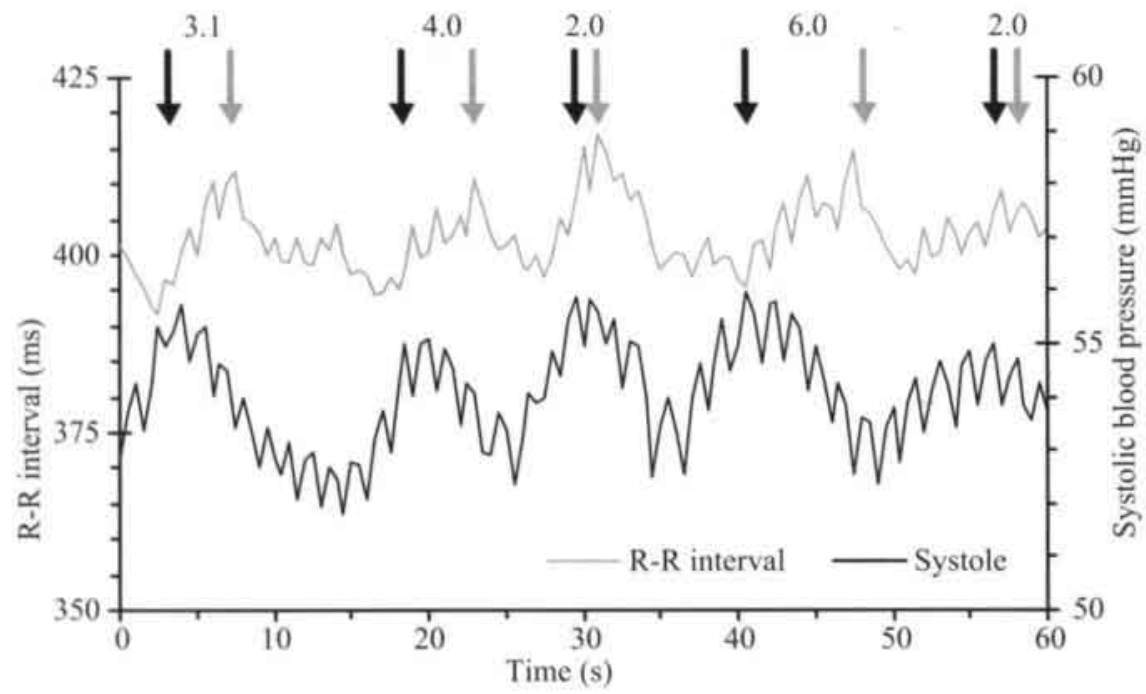

Figure 5. Temporal relationship between SBP and R-R interval of the same infant as in Figure I. R-R interval (left $y$ axis) and systolic blood pressure (right $y$ axis) values are shown as a function of time ( $x$ axis). Time is expressed in seconds (s). Beside HF fluctuations, related to the respiratory frequency, approximately five LF fluctuations in this trace of $60 \mathrm{~s}$ can be seen from the signals, corresponding with a LF peak of approximately $0.08 \mathrm{~Hz}$. LF fluctuations of BP precede BR changes in R-R intervals with a time delay varying between $2-6 s$ (period between arrows). 
the temporal relationship between the signals, illustrated in Figure 5, strongly suggests LF fluctuations in SBP precede fluctuations in R-R intervals for about the calculated time delay of approximately $4 \mathrm{~s}$. It is highly unlikely that at LF, R-R interval changes precede arterial pressure changes with a latency of $14.3-3.8=10.5 \mathrm{~s}$.

In human adults, a LF peak at $0.10 \mathrm{~Hz}$ and a phase difference of approximately $60-70^{\circ}$ (pressure leads R-R interval) are calculated $4,6,34$. This corresponds with a far lesser time delay between BP and R-R interval fluctuations in human adults, approximately $2 \mathrm{~s}$. These findings suggest that preterm infants have a longer time delay of the baroreceptor loop. In case of $\mathrm{HR}$ frequencies of $2.5 \mathrm{~Hz}$ and respiratory frequencies of approximately $0.8 \mathrm{~Hz}$, taking into account the closed loop properties, interpretation of the phase shift in the HF range is not possible. We did not found a consistent positive or negative phase difference at HF. It is not clear whether HF fluctuations in SBP lead or lag fluctuations in R-R intervals (Figure 5).

\section{Frequency domain (tranfer function) versus time domain assessment of BR gain:}

Transfer function can be used effectively to estimate the BR sensitivity provided that phase relationship and coherence between variables are considered ${ }^{4,19-21}$. We found a strong and significant correlation between frequency specific transfer function gain in the LF band and a time domain estimate for total variability gain. To our knowledge this has never been demonstrated before. Time-domain analysis of variability refers to statistical calculations of adjacent (R-R interval values) intervals, e.g. modifications of the mean, SD, sorting and selecting, and slope changes ${ }^{35}$. An example of a simple time domain index is the SD of $(\mathrm{R}-\mathrm{R})_{\mathrm{i}}$ intervals within a period. This index has been thought to result from the dynamic balance between the sympathetic and parasympathetic components of the autonomic nervous system ${ }^{36}$. The SD of $(\mathrm{R}-\mathrm{R})_{\mathrm{i}}$ intervals or SBP-toSBP values within a certain period reflects the total variance of the signal. Power spectral density analysis provides the basic information of how power (variance) distributes as a function of frequency. Because LF power has been demonstrated to be dominant in both R-R and SBP frequency analysis, it is not surprising that the BR gain derived from cross-spectral analysis correlates strongly with a time domain estimate for total variability gain. In other words BR gain/sensitivity in the LF range can be estimated by a simple time domain index.

Methodological considerations: The small size of the group and the range in GA in our study population explains the lack of evidence of the effect of GA, PCA and BW on any of the spectral power values or the transfer function parameters in the study group. Another possible limitation of the study might be the effect of antenatal administration of steroids on glucocorticoid-dependent maturation of the BR and fetal HR variability. 
Antenatal glucocorticoids decrease the sensitivity of the BR after birth in a preterm delivered sheep model ${ }^{37}$. In human fetuses antenatal glucocorticoids transitorily lower short- and long-term fetal heart rate variability for 1-3 days after administration and normalize at day $4^{38}$. Although the period between antenatal glucocorticoid administration and postnatal measurement was at least 5 days in this study, it is possible that our postnatal results might reflect the effect of antenatal steroids rather than normal development of the preterm infant. Another issue concerns the physiological significance of heart rate variability with respect to autonomic nervous system and maturation. Inasmuch as HR variability reflects the response of the sinoatrial node to the effects of the autonomic nervous system, it is possible that specific receptors of the sinoatrial node are not mature at the same time in preterm infants. HR variability may thus not necessary reflect the direct input of the autonomic system.

\section{Conclusion}

In conclusion, we studied cardiovascular variability in stable preterm infants by means of cross-spectral analysis of R-R interval and SBP fluctuations. We found high LF/HF ratios in both R-R interval series and SBP series in preterm infants. The magnitude of the transfer function (BR gain or sensitivity) between R-R interval and SBP is higher in the LF band than in the HF band. This suggests that a high LF/HF ratio, reflecting an altered sympathovagal balance, might be a result of increased sympathetic activity. Our findings suggest a longer time delay of LF fluctuations between BP and R-R interval in preterm infants (approximately $4 \mathrm{~s}$ ) than in human adults (approximately $2 \mathrm{~s}$ ). The cross spectral signal analysis is an interesting technique because it could offer a test for tracking the development of the sympathetic system.

\section{Acknowledgement}

The authors thank Ben Janssen (Department of Pharmacology; Cardiovascular Research Institute of Maastricht, University of Maastricht, Maastricht, The Netherlands) for his critical review of the manuscript. 


\section{References}

1. Akselrod S, Gordon D, Madwed JB, Snidman NC, Shannon DC, Cohen RJ 1985 Hemodynamic regulation: investigation by spectral analysis. Am J Physiol 249:H867-H875

2. Penáz J 1978 Mayer waves: history and methodology. Automedica 2: 135-141

3. Task Force of the European Society of Cardiology and the North American Society of pacing and electrophysiology 1996 Heart rate variability: standards of measurement, physiological interpretation, and clinical use. Circulation 93:1043-1065

4. de Boer RW, Karemaker JM, Strackee J 1987 Hemodynamic fluctuations and baroreflex sensitivity in humans: a beat-to-beat model. Am J Physiol 253:H680-H689

5. Saul JPh, Berger RD, Albrecht P, Stein SP, Hui Chen M, Cohen RJ 1991 Transfer function analysis of the circulation: unique insights into cardiovascular regulation. Am J Physiol 261:H1231-H1245

6. Cooke WH, Hoag JB, Crossman AA, Kuusela TA. Tahvanainen KUO, Eckberg DL 1999 Human responses to upright tilt: a window on central autonomic integration. J Physiol 517.2: 617-628

7. ten Voorde BJ, Ree EJ, Hack WWM, Bergschneider VM, Hoekstra BPT, Faes ThJC, Witte H, Rother M 1992 High-frequency and low-frequency heart-rate fluctuations analysis in newborns - A review of possibilities and limitations. Basic Res Cardiol 87:193-204

8. van Ravenswaay-Arts C, Hopman J, Kollée L, Stoelinga G, van Geijn H 1994 Spectral analysis of heart rate variability in spontaneously breathing very preterm infants. Acta Paediatr 83:473-480

9. Chatow U, Davidson S, Reichman BL. Akselrod S 1995 Development and maturation of the autonomic nervous system in premature and full-term infants using spectral analysis of heart rate fluctuations. Pediatr Res 37:294-302

10. Scher MS, Steppe DA. Dokianakis G, Sun M, Guthrie RD, Sclabassi RJ 1994 Cardiorespiratory behaviour during sleep in full-term and preterm neonates at comparable postconceptional term ages. Pediatr Res 36:738-744

11. Mazurksky JE, Birkett CL, Bedell KA, Ben-Haim SA, Segar Л 1998 Development of baroreflex influences on heart rate variability in preterm infants. Early Hum Develop 53:37-52

12. Kloosterman GJ 1970 On intrauterine growth. Int J Gynaec Obst 8:895-912

13. Prechtl HFR 1974 The behavioural states of the newborn infant (a review). Brain Res 76:185-212

14. Jaffe RS, Fung DL 1994 Constructing a heart rate variability analysis system. J Clin Monit 10:45-58

15. Akselrod S 1995 Components of heart rate variability: basic studies. In: Malik M, Camm AJ (eds) Heart rate variability. Futura Publishing Company, New York, pp 147-163

16. Welch PD 1967 The use of fast Fourier transform for the estimation of power spectra: a method based on time averaging over short, modified periodograms. IEEE Trans Audio and Electroacoust 15:70-73

17. Bingham C, Godfrey MD, Tukey JW 1967 Modern techniques of power spectrum estimation. IEEE Trans Audio and Electroacoust 15:56-66

18. de Boer RW, Karemaker JM. Strackee J 1985 Relationships between short-term blood pressure fluctuations and heart rate variability in resting subjects: a spectral approach. Med Biol Eng Comput 23:352-358

19. Head GA, Lukoshkova EV, Burke SL, Malpas SC, Lambert EA, Janssen BJA 2001 Comparing spectral and invasive estimates of baroreflex gain. IEEE Eng Med Biol Mag 20(2):43-52

20. Robbe HWJ, Mulder LJM, Rüddel H, Langewitz WA, Veldman JBP, Mulder G 1987 Assessment of baroreceptor reflex sensitivity by means of spectral analysis. Hypertension 10:538-543

21. Honzíková N, Fišer B, Honzík J 1992 Noninvasive determination of baroreflex sensitivity in man by means of spectral analysis. Physiol Res 41:31-37

22. Smeulders M. Agbeko R, de Jong W. Koolen AMP 1994 Modulation of heart rate by the arterial baroreceptor reflex in neonates 1994. Pediatr Res 212:38A 
23. Metsailä T, Siimes A, Antila K, Välimäki I 1993 Association of breathing movements to the variability of heart rate and blood pressure in foetal lambs. Acta Physiol Scand 147:213-219

24. Blanco CE, Dawes GS, Hanson MA, McCooke HB 1988 Carotid baroreceptors in fetal and newborn sheep. Pediatr Res 24:342-346

25. Giddens DP, Kitney R1 1985 Neonatal heart rate variability and its relation to respiration. J Theor Biol 113:759-780

26. Saul JPh, Rea RF, Eckberg DL, Berger RD, Cohen RJ 1990 Heart rate and muscle sympathetic nerve variability during reflex changes of autonomic activity. Am J Physiol 258:H713-H721

27. Fritsch JM, Eckberg DL, Graves LD, Wallin BG 1986 Arterial pressure ramps provoke linear increases of heart period in humans. Am J Physiol 251:R1086-R1090

28. Sanders JS, Ferguson DW, Mark AL 1988 Arterial baroreflex control of sympathetic nerve activity during elevation of blood pressure in normal man: dominance of aortic baroreflexes. Circulation 77:279-288

29. Clayton RH, Bowman AJ, Ford GA, Murray A 1995 Measurement of baroreflex gain from heart rate and blood pressure spectra: a comparison of spectral estimation techniques. Physiol Meas 16:131139

30. Drouin E, Gourmay V, Calamel J, Mouzard A, Rozé JC 1997 Assessment of spontaneous baroreflex sensitivity in neonates. Arch Dis Childhood 76:F108-F112

31. Wakatsuki A, Murata Y, Ninomiya Y, Masaoka N, Tyner JG, Kutty KK 1992 Physiologic baroreceptor activity in the fetal lamb. Am J Obstet Gynecol 167:820-827

32. Grönland JU, Antila KJ, Siimes ASI, Metsälä T, Oja R, Tuominen J, Välimäki IAT 1989 Betaadrenergic control and inter-relationships between heart rate and blood pressure in neonatal lambs. Med Biol Eng Comput 27:163-170

33. Grönland JU, Kalli ST, Siimes ASI, Sydänmaa M, Antila KJ, Vallimäki IAT 1991 Do beta-adrenergic blockade and sleep state affect cardiorespiratory control in neonatal lambs? Multivariate autoregressive modeling approach. Pediatr Res 29:272-277

34. Baselli G, Cerutti S, Civardi S, Liberati D, Lombardi F, Malliani A, Pagani M 1986 Spectral and cross-spectral analysis of heart rate and arterial blood pressure variability signals. Comput Biomed Res 19:520-534

34. Parer WJ, Parer JT, Holbrook RH, Block BSB 1985 Validity of mathematical methods of quantitating fetal heart rate variability. Am J Obstet Gynecol 153:402-409

35. Cabal LA, Siassi B. Zanini B. Hodgman JE, Hon EE 1980 Factors affecting heart rate variability in preterm infants. Pediatrics 65:50-56

36. Segar JL, Lumbers ER, Nuyt AM, Smith OJ, Robillard JE 1998 Effect of antenatal glucocorticoids on sympathetic nerve activity at birth in preterm sheep. Am J Physiol 274:R160-170

37. Derks JB, Mulder EJH, Visser GHA 1995 The effects of maternal betamethasone administration on the fetus. Br J Obstet Gynaecol 102:40-46 


\section{Chapter 7}

\section{Cardiovascular autonomic regulation in preterm infants: the effect of atropine}

Peter Andriessen, Ben JA Janssen, Ralph C.M. Berendsen, Sidarto Bambang Oetomo, Pieter F.F. Wijn, Carlos E. Blanco

Pediatric Research - In Press

Published online before print October 6, 2004

DOI: $10.1203 / 01 . P D R .0000145257 .75072 . B B$ 


\section{Abstract}

To study cardiovascular autonomic control, we assessed the effect of atropine on heart rate (HR) and blood pressure (BP) variability in 12 preterm infants (range, 26-32 wk) before intubation for respiratory insufficiency. Spectral power analysis of R-R interval and systolic BP (SBP) series were estimated in a low frequency (LF: 0.04-0.15 Hz) and high frequency (HF: 0.4-1.5 Hz) band and evaluated for a 10-min period before and a 10 -min period after atropine sulfate $(0.01 \mathrm{mg} / \mathrm{kg})$. Baroreceptor reflex (BR) functioning was estimated using transfer function analysis at LF (coherence, gain and phase).

Atropine resulted in a significant $12 \%$ increase in steady-state HR $(\mathrm{p}<0.01)$ and unchanged SBP. For R-R interval series, the total spectral power decreased six-fold ( $p$ $<0.01$ ) which was predominantly due to a reduction in the LF band (sixteen-fold, p < $0.01)$. In contrast, we observed a significant increase $(+25 \%, \mathrm{p}<0.05)$ in total spectral power of SBP series partly due to an increase in HF power. The LF power of SBP series was not altered. The median LF transfer gain (BR sensitivity) between SBP and R-R interval decreased from 4.2 to $1.4 \mathrm{~ms} / \mathrm{mmHg}(\mathrm{p}<0.01)$ after atropine. The $\mathrm{LF}$ phase relationship (BP leads R-R interval fluctuations by approximately $4 \mathrm{~s}$ ) was not changed after atropine.

In conclusion, even in preterm infants in distress, atropine modulates HR and BP variability, suggesting that BR-mediated parasympathetic control of $\mathrm{HR}$ is of significance for cardiovascular control at that age.

\section{Abbreviations}

$\begin{array}{ll}\text { BP } & \text { blood pressure } \\ \text { BR } & \text { baroreceptor reflex } \\ \text { HF } & \text { high frequency }(0.4-1.5 \mathrm{~Hz}) \\ \text { HR } & \text { heart rate } \\ \text { IQR } & \text { interquartile range }(\mathrm{P} 25-\mathrm{P} 75) \\ \text { LF } & \text { low frequency }(0.04-0.15 \mathrm{~Hz}) \\ \text { R-R } & \text { R-R interval length } \\ \text { SBP } & \text { systolic blood pressure }\end{array}$




\section{Introduction}

The baroreceptor reflex (BR) is the most important regulatory mechanism in the shortterm control of heart rate (HR) and blood pressure (BP). The BR buffers sudden changes in systemic BP by adapting HR and peripheral vascular resistance. These responses of $\mathrm{HR}$ and vascular resistance are mediated by the efferent parasympathetic and sympathetic limb of the BR. As both systems are involved, studies on the BR give information on autonomic cardiovascular regulation ${ }^{1,2}$.

Data about the ontogeny of the BR and functional maturation in the human infant are limited. This is partly caused by the limited experimental (pharmacological or mechanical) possibilities to challenge the BR in neonates. Passive head-up tilt test has been applied to neonates to measure responses in BP, HR and limb blood flow to body tilting. In preterm infants (26-38 wk gestation), passive head-up tilt resulted in significant vasoconstriction of the lower limb with a slight fall in aortic BP and unchanged $\mathrm{HR}^{3}$. The inadequate ability to maintain $\mathrm{BP}$ and the lack of a tachycardia suggest that preterm infants lack the full integrated BR response as seen in adults. In term infants, however, a fall in systolic BP (SBP) was observed in conjunction with a tachycardia and a fall in limb blood flow, suggesting the presence of active reflex vasoconstriction ${ }^{4}$. In a recent study, BR maturation was studied longitudinally in preterm infants, using a beat-to-beat analysis of R-R interval series and noninvasively measured SBP5 . BR sensitivity (the R-R interval change in milliseconds per millimeter of mercury of SBP change) at birth was lower in preterm infants than in term babies and increased in postnatal life. This study, however, did not answer to what extent the parasympathetic and sympathetic system contribute to BR functioning. Previously, we showed the feasibility to use cross-spectral analysis to estimate BR functioning from spontaneous HR and BP fluctuations in stable preterm infants ${ }^{6}$. With this technique, we found indications of a dominant role of the sympathetic system in stable preterm infants in the first days after birth.

Knowledge about the developmental aspects of the BR relies almost exclusively on animal studies. In animal studies it is shown that the BR is impaired in the fetus and the newborn and develops during further postnatal life ${ }^{7-9}$. The impairment in BR function is due to maturational changes in the autonomic pathways ${ }^{10}$. In sheep, the sympathetic and parasympathetic control systems mature at different rates during fetal and neonatal development ${ }^{11}$. The parasympathetic system exerts very little tone on the resting fetal HR before term gestation. Blockade of the parasympathetic system with atropine did not change HR in the premature fetuses. Only a significant HR increase was observed in the mature fetuses without significant changes in systemic $\mathrm{BP}^{12}$. These observations suggest a progressive maturation of the parasympathetic nervous system during fetal 
development. In contrast to the negligible parasympathetic tone exerted on the fetal circulatory functions prior to maturity, the sympathetic control begins in early fetal life and increases with the progression of gestation ${ }^{12}$. Blockade of the sympathetic system has profound effects on HR in all fetuses regardless of gestational age. In sheep, arterial $\mathrm{BP}$ and HR are depressed by ganglionic blockade in the newborn (1-3 days) but not in older lambs ${ }^{13}$. These findings suggest that sympathetic tone is high during the transitory phase in the early postnatal period and decreases in later life.

In summary, human and animal data suggest that the development of the parasympathetic system parallels the maturation of BR function with gestational age and postnatal life. In addition an activated sympathetic system is dominantly present in the immediate postnatal period and subsequently decreases over time. Whether vagal tone is able to modulate BR in sympathetically activated preterm infants in the transitory phase after birth is currently unknown. We therefore evaluated cardiovascular autonomic regulation by spectral power analysis in preterm infants in response to selective parasympathetic blockade. Atropine was administered as part of the standard neonatal intensive care management before endotracheal intubation for respiratory insufficiency.

The objective of this study was to determine the contribution of the parasympathetic system to BR functioning by comparing changes in spectral power of R-R interval and SBP series, and changes in coherence, gain and/or phase as assessed by transfer function analysis (between SBP and R-R interval fluctuations) before and after muscarinic blockade by atropine.

\section{Methods}

Subjects: The study group consisted of 12 preterm infants. The relevant clinical data are shown in Table 1. Before labour, 10 women received antenatal steroids and 9 women were on tocolytic (ritodrine intravenously) therapy. All infants were appropriate-forgestational age, according to the Dutch growth charts ${ }^{14}$. All infants were studied once, around the time of atropine administration just before the intubation procedure, in the first week of life. Administration of atropine sulfate $(0.01 \mathrm{mg} / \mathrm{kg}$, intravenously) is part of our regular neonatal intensive care management before endotracheal intubation. The reason for endotracheal intubation was neonatal respiratory distress syndrome $(n=5)$, apnea $(n=5)$ and infection $(n=2)$. The median age of atropine administration was $36 \mathrm{~h}$ after birth. None had echoencephalographic evidence of cerebral hemorrhage or parenchymal lesions. Echocardiography revealed no structural abnormalities. In 4 subjects a patent ductus arteriosus was medically closed $30-120 \mathrm{~h}$ before the study. All 
Table 1: Clinical characteristics of the study population at time of the measurements

Gestational age (weeks)

Birth weight (g)

Umbilical arterial $\mathrm{pH}$

Median (IQR) 5-min Apgar score

Median (IQR) postnatal age at time of study (hrs)

Carbon dioxide $(\mathrm{mmHg})$

Oxygen need (\%)

Hematocrit
$27.8 \pm 2.3$

$1081 \pm 514$

$7.28 \pm 0.09$

$8 \quad(7-9)$

$36 \quad(12-150)$

$46 \pm 13$

$42 \pm 18$

$0.43 \pm 0.07$

Data expressed as mean $\pm \mathrm{SD}$ or median (IQR).

infants were judged as cardiovascular stable without need of cardiotropic drugs (dopamine, dobutamine) or volume expanders at the time of the study. All subjects' respiration was supported by nasal continuous positive airway treatment, with a mean airway pressure of $4 \mathrm{~cm} \mathrm{H}_{2} \mathrm{O}$. Four infants were on caffeine therapy with serum concentration levels between 10 and $20 \mathrm{mg} / \mathrm{l}$, and all but 3 received antibiotic therapy. Informed consent was obtained from the parents of each infant. The local Ethics Committee approved the study.

Data acquisition and analysis: A bipolar chest lead of the surface ECG and the transthoracic electric impedance waveforms were recorded by Hewlett Packard neonatal monitor type Merlin (Waltham, USA). The respiration rate was derived from the transthoracic electric impedance waveform. The arterial BP was measured invasively through a 4-French catheter in the lower aortic position used for routine monitoring of vital functions and intensive care management. A $0.5 \mathrm{ml} / \mathrm{h}$ infusion of heparinized physiological saline solution was continuously flushed through the catheter. The sample frequency was $512 \mathrm{~Hz}$ for the ECG signals and $256 \mathrm{~Hz}$ for the arterial BP signals. Recordings were made before the intubation procedure in the prone position. The total registration time was $30 \mathrm{~min}$ : $15 \mathrm{~min}$ baseline and $15 \mathrm{~min}$ after atropine. Figure 1 shows the traces of R-R interval and SBP series during the signal acquisition period in a subject. We divided the baseline period into three 5-min periods and found no significant differences in mean R-R interval or SBP between these periods. A 10-min period was selected from the first and second 5-min period to represent the control state and was subjected for spectral power analysis. Likewise, the post-atropine period was divided into three 5 -min periods. After $5 \mathrm{~min}$ of atropine administration, no additional 


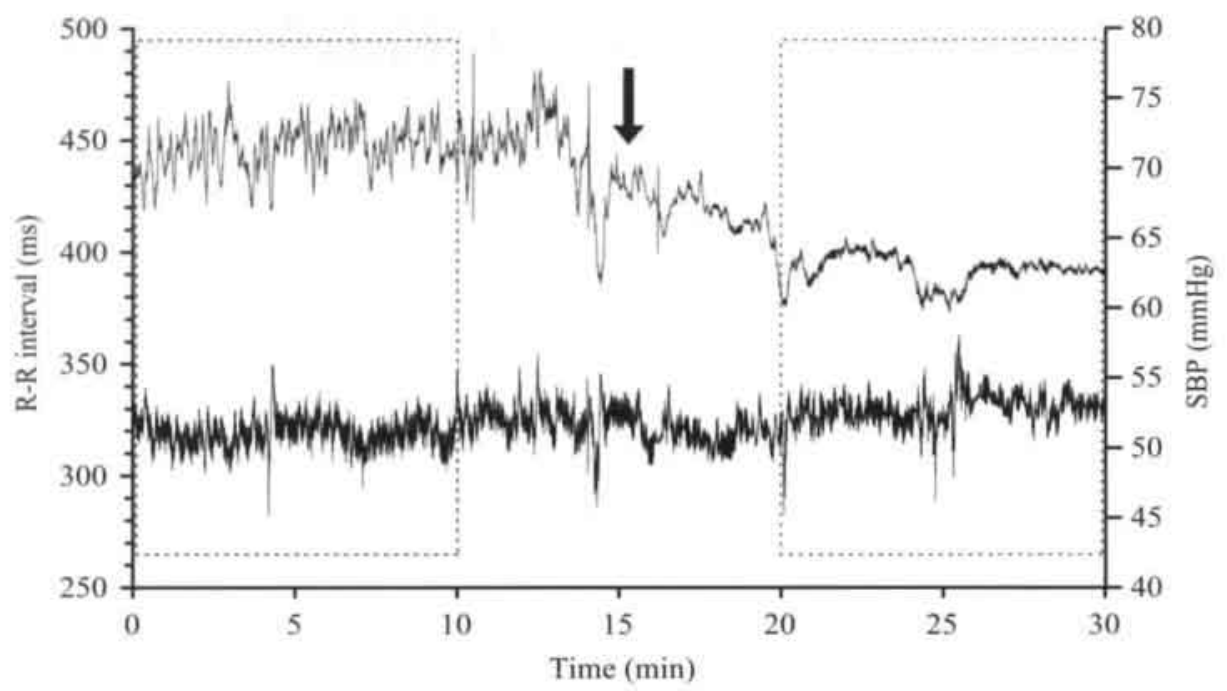

Figure 1. Example of a $30-$ min trace of $R-R$ interval and SBP series in a preterm infant. The arrow (at time $15 \mathrm{~min}$ ) indicates atropine administration. Note the decrease in R-R interval and variability (upper trace) after atropine. In contrast, no change in SBP and variability (lower trace) is observed. The 2 boxes indicate two 10-min periods (control state and steady atropine state) which were used for further spectral power analysis.

changes in R-R interval values were observed. The period 5-15 min after atropine administration was considered to be in a steady-state. These data were pooled to ensemble one 10-min period to represent the atropine state and subjected for spectral power analysis.

Spectral power analysis of $R-R$ interval series and SBP series: With respect to details of the spectral power analysis we refer to our previous report ${ }^{6}$. Briefly, $\mathrm{R}$ waves were detected from the ECG and an unevenly spaced R-R interval sequence was created. The $\mathrm{R}-\mathrm{R}$ interval sequence was resampled at $4 \mathrm{~Hz}$ in order to obtain an equidistantly spaced time series. R-R artefacts were identified when the R-R interval was less than 200 or more than $500 \mathrm{~ms}$. Missing R-R intervals were linearly interpolated. The amount of artefact reduction was less than $2 \%$ per subject. SBP was identified from peak detection of the arterial BP signal, resulting in an unevenly spaced "systogram". The "systogram" was converted into an equidistantly spaced time series using the same resampling method as used for the R-R interval series. Spectral power of the various frequency components of SBP and R-R interval series was calculated using a moving fast Fourier transformation of 64-s segment length (256 points), providing a spectrum every $0.25 \mathrm{~s}$ with a resolution of $0.0156 \mathrm{~Hz}$. Within each 10 -min period (control state, postatropine), the resulting spectra were subjected to ensemble one average spectral density 
curve. The data acquisition software package was developed at the department of Clinical Physics of our hospital in collaboration with the department of Physics of the Eindhoven University of Technology, The Netherlands.

Unlike the consensus for spectral analysis in human adult ${ }^{15}$, there is no agreement in spectral band definitions for neonatal studies ${ }^{16-20}$. As the neonatal HR and respiration rates differ from that of the adult, neonatal studies likely require a different HF definition. Two frequency bands were defined, as indicated in Figures 2 and 3:1) the low frequency band (LF: 0.04-0.15 Hz) reflecting BR activity and 2) the high frequency band (HF: 0.4-1.5 Hz) reflecting the range of respiratory rate in preterm infants. The very low frequency band $(0-0.04 \mathrm{~Hz})$ was discarded to avoid possible contribution of slow trend artefacts. The total frequency (TF) band of interest was the range between 0.04 and $1.5 \mathrm{~Hz}$. Spectral power was calculated in each defined frequency band. The values for spectral power are presented in units of milliseconds ${ }^{2}$ (for $\mathrm{R}-\mathrm{R}$ interval series) and $\mathrm{mmHg}^{2}$ (for BP series).

Transfer function analysis: In addition to the spectral density power, the transfer function analysis (coherence, transfer gain and phase) was calculated, as previously described in detail $6,21,22$. Briefly, transfer gain reflects the degree to which the input signal (SBP) amplitude becomes manifest in the output signal ( $R-R$ interval) amplitude at a discrete frequency. At LF, transfer gain is an estimate for BR gain/sensitivity 22,23 . The phase difference (degrees or seconds) indicates the lead or lag of one signal with respect to the other at a discrete frequency. A negative phase relation indicates SBP fluctuations lead R-R interval changes. In addition to these calculations, the coherence function was computed to assess the amount of linear coupling between SBP and R-R interval series in the frequency domain. The coherence function, which ranges from 0 (no relationship) and 1 (linear relationship) was used to assess the statistically reliability of the transfer function at each frequency. Transfer gain and phase were assessed in each frequency band at the frequency of highest coherence value ${ }^{6}$.

Statistical analysis: For each period, the autospectra densities were subjected to ensemble averaging. Because spectral power between subjects do not display a normal distribution, the spectral power values of R-R interval and SBP series and transfer function analysis (frequency, coherence, gain, and phase) are expressed as (group) median and inter quartile range (IQR). Comparisons between the control and atropine state were made with a two-sided paired Wilcoxon sign rank test for non-parametric data. The influence of gestational age and use of caffeine on spectral power values and transfer function variables was studied by linear regression analysis, using the method of least squares, and ANOVA. Statistical significance was accepted at $\mathrm{p}$ values $<0.05$. 


\section{Results}

The median R-R interval decreased from 422 to $378 \mathrm{~ms}$ ( $p<0.01$ ), equivalent to a $12 \%$ increase in HR after atropine. Table 2 shows the results of the spectral power values for $\mathrm{R}-\mathrm{R}$ interval series and respiratory frequency in response to atropine administration. The median total spectral power decreased a six-fold from 135 to $22 \mathrm{~ms}^{2}$ after atropine ( $p<0.01$ ). Remarkably, the spectral power was much more decreased within the LF (sixteen-fold, $\mathrm{p}<0.01$ ) than within the HF band (only three-fold, $\mathrm{p}<0.05$ ). All subjects showed a similar pattern with a decrease in total spectral power and a predominant decrease in LF. In Figure 2 the individual spectral density curves and the (group) median spectral density curve of R-R interval series are shown for the control state (top) and after atropine (bottom). Atropine did not have a significant effect on the respiratory frequency of the subjects (control state, median $0.98 \mathrm{~Hz}$; atropine state, median 0.91 $\mathrm{Hz}$ ). The respiratory frequency range was within the $\mathrm{HF}$ band. The median spectral $\mathrm{LF} / \mathrm{HF}$ ratio decreased from 7.9 to 1.3 after atropine $(\mathrm{p}<0.01)$.

SBP remained unchanged after atropine administration. Table 3 shows the results of spectral power values for SBP series in response to atropine administration. Following atropine total spectral power increased in 9 subjects. The median total spectral power increased from 4.1 to $5.2 \mathrm{mmHg}^{2}$ after atropine $(+25 \%, \mathrm{p}<0.05)$. Remarkably, no change in LF spectral power was observed. Following atropine the HF spectral power increased in 11 subjects. The median HF spectral power increased from 1.4 to 1.7 $\mathrm{mmHg}^{2}(+20 \%, \mathrm{p}<0.01)$.

Table 2: Spectral power values for $\mathrm{R}-\mathrm{R}$ interval series and respiration rate

\begin{tabular}{lccl}
\hline & \multicolumn{1}{c}{ control state } & \multicolumn{1}{c}{ atropine } & Wilcoxon \\
\hline R-R interval & $422(399-439)$ & $378(353-402)$ & $\mathrm{p}<0.01$ \\
Total power & $135(59-172)$ & $22(8-52)$ & $\mathrm{p}<0.01$ \\
LF power & $99(34-155)$ & $6(3-35)$ & $\mathrm{p}<0.01$ \\
HF power & $15(7-21)$ & $5(3-18)$ & $\mathrm{p}<0.05$ \\
Respiratory frequency & $0.98(0.83-1.19)$ & $0.91(0.84-1.14)$ & $\mathrm{ns}$ \\
\hline
\end{tabular}

The $\mathrm{R}-\mathrm{R}$ interval is expressed in $\mathrm{ms}$ and spectral power values in $\mathrm{ms}^{2}$. The respiratory frequency is expressed in $\mathrm{Hz}$. Data are expressed as median and inter quartile range between brackets. The total spectral power was computed for the frequency band between 0.04 and $1.5 \mathrm{~Hz}$. The low frequency (LF) band was defined between 0.04 and $0.15 \mathrm{~Hz}$, the high frequency (HF) band between 0.4 and $1.5 \mathrm{~Hz}$. The p values (paired Wilcoxon sign rank test) are expressed in the last column, ns refers to not significant. 

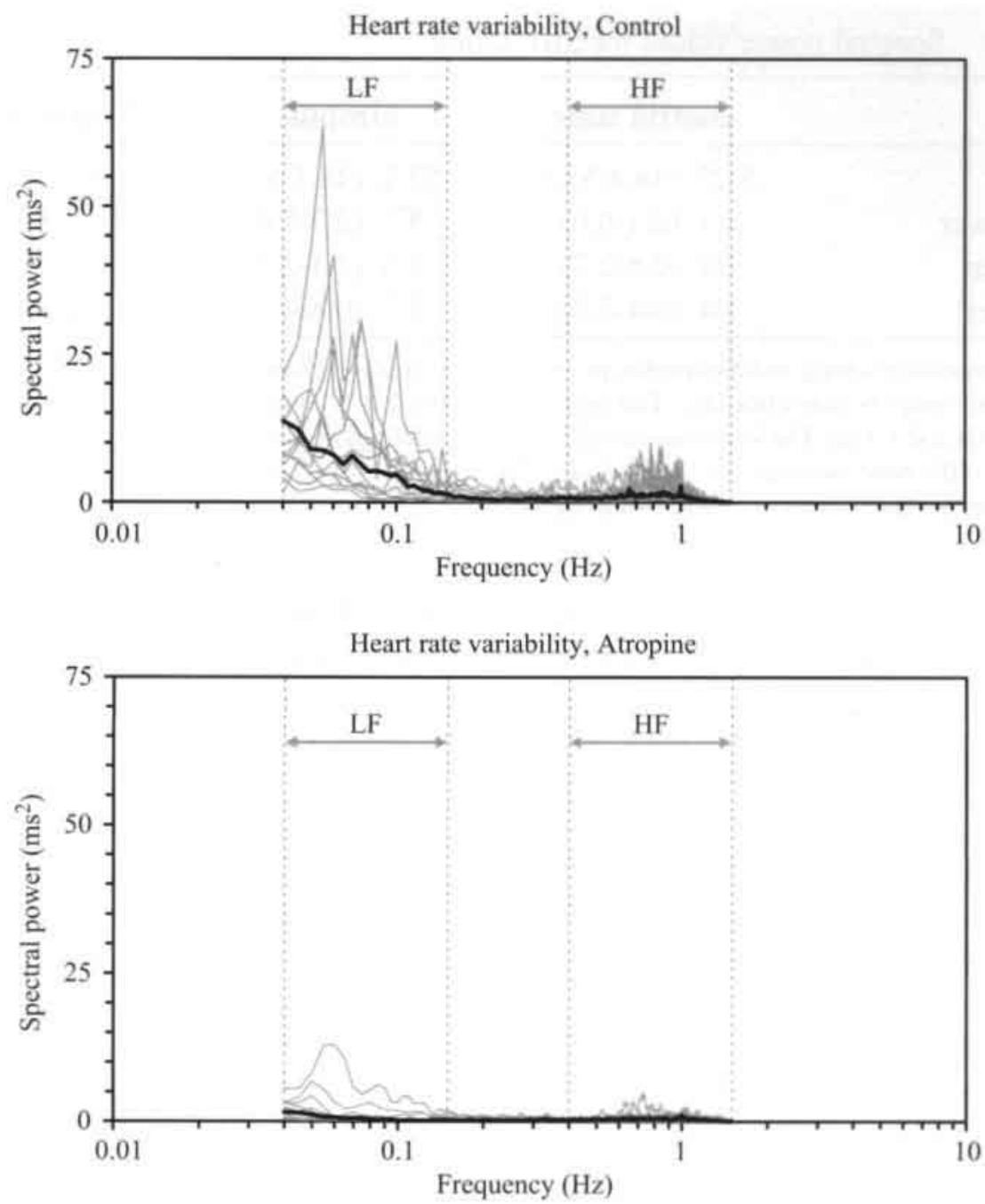

Figure 2. Change in spectral power for $R-R$ interval series after atropine administration. The individual spectral density curves and the (group) median spectral density curve of R-R interval series are shown for the control state (top) and after atropine (bottom). The spectral power $\left(\mathrm{ms}^{2}\right)$ is distributed as a function of frequency $(\mathrm{Hz})$. The $x$ axis is logarithmic scaled in order to visualize the LF band better. The spectral density curves of the individual subjects are presented as thin lines and the median value as a bold line. The vertical lines indicate the boundaries of the low frequency band (LF: 0.04-0.15 Hz) and high frequency band (HF: $0.4-1.5 \mathrm{~Hz}$ ). Note that the spectral energy is particularly distributed in the LF and $\mathrm{HF}$ band. In the LF band individual peaks can be observed corresponding with baroreceptor reflex activity. The increase of spectral power in the HF band corresponds to respiratory activity. The total spectral power is decreased six-fold, predominantly in the LF band (sixteen-fold) and much less in the HF band (three-fold). 
Table 3: Spectral power values for SBP series

\begin{tabular}{lccl}
\hline & control state & atropine & Wilcoxon \\
\hline SBP & $51.5(48.4-53.8)$ & $52.1(48.5-52.5)$ & $\mathrm{ns}$ \\
Total power & $4.1(2.1-6.0)$ & $5.2(2.7-7.6)$ & $\mathrm{p}<0.05$ \\
LF power & $1.8(0.8-2.2)$ & $1.8(1.1-2.9)$ & $\mathrm{ns}$ \\
HF power & $1.4(0.4-2.9)$ & $1.7(0.8-4.9)$ & $\mathrm{p}<0.01$ \\
\hline
\end{tabular}

SBP is expressed in $\mathrm{mmHg}$ and the spectral power values in $\mathrm{mmHg}^{2}$. Data are expressed as median and inter quartile range between brackets. The total spectral power was computed for the frequency band between 0.04 and $1.5 \mathrm{~Hz}$. The low frequency (LF) band was defined between 0.04 and $0.15 \mathrm{~Hz}$, the high frequency (HF) band between 0.4 and $1.5 \mathrm{~Hz}$. The p values (paired Wilcoxon sign rank test) are expressed in the last column, ns refers to non significant.

In Figure 3 the individual spectral density curves and the (group) median spectral density curve of SBP series are shown for the control state (top) and after atropine (bottom).

Table 4 shows the result of the transfer function analysis. The control state showed the highest coherence values (median, 0.43; IQR, 0.28-0.55) in the LF band at a median frequency of $0.08 \mathrm{~Hz}$. The control state showed the highest coherence values in the $\mathrm{HF}$ band (median, 0.73; IQR, 0.62-0.82) at a median frequency of $0.81 \mathrm{~Hz}$. In the control state, LF gain (median $4.2 \mathrm{~ms} / \mathrm{mmHg}$ ) was lower than HF gain (median $8.3 \mathrm{~ms} / \mathrm{mmHg}$, $p<0.05$ ). In the control state, LF phase (median $-3.7 \mathrm{~s}$ ) was significantly different from HF phase (median $+0.4 \mathrm{~s}, \mathrm{p}<0.05$ ).

The median LF transfer gain (BR sensitivity) decreased significantly from 4.2 to $1.4 \mathrm{~ms} / \mathrm{mmHg}$ after atropine $(\mathrm{p}<0.01)$. The HF transfer gain did not change after atropine. In both frequency bands, atropine did not change the phase relation between SBP and R-R interval fluctuations. Because coherence values above 0.5 are (arbitrarily) assumed to be of statistical significant value using physiological signals and might limit the value of the transfer function ${ }^{23}$, we performed a subanalysis of the LF transfer function data in 7 subjects with coherences values $>0.5$. The median LF gain decreased significantly from 4.4 (IQR, 3.1-5.7) to 1.0 (IQR, 0.8-1.7) $\mathrm{ms} / \mathrm{mmHg}(\mathrm{p}<0.05)$. The median LF phase did not significantly change after atropine and remained approximately $-4 \mathrm{~s}$. The (negative) phase relation in the LF band in the control state indicates that SBP fluctuations lead R-R interval changes.

No significant influence could be demonstrated of gestational age and use of caffeine on R-R interval and SBP series, spectral power analysis and transfer function analysis. 


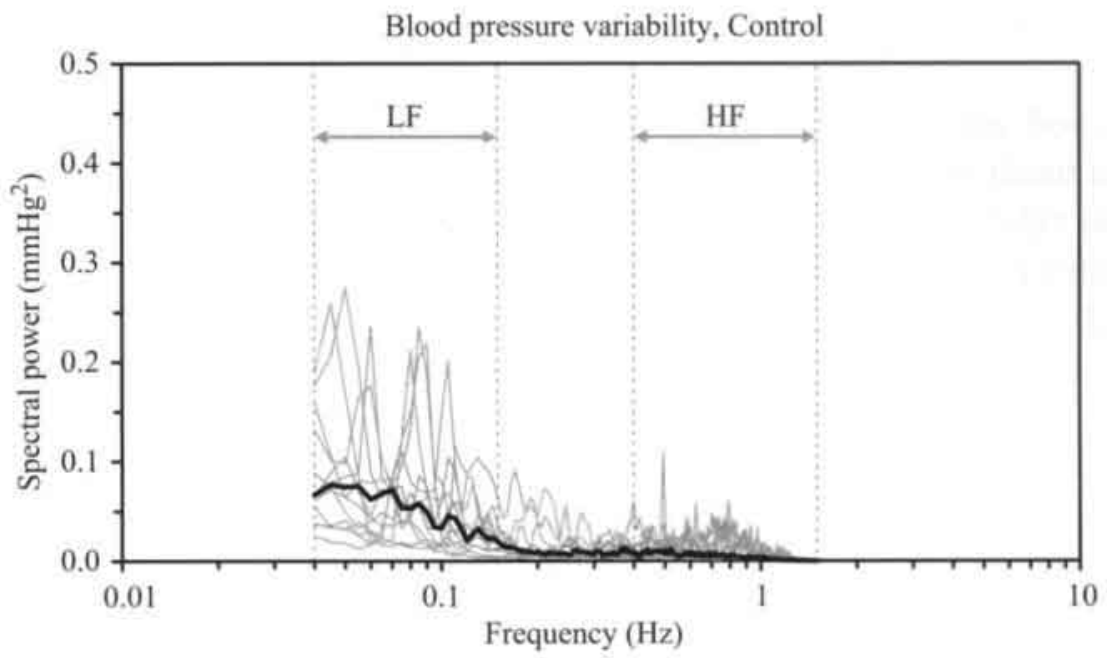

Blood pressure variability, Atropine

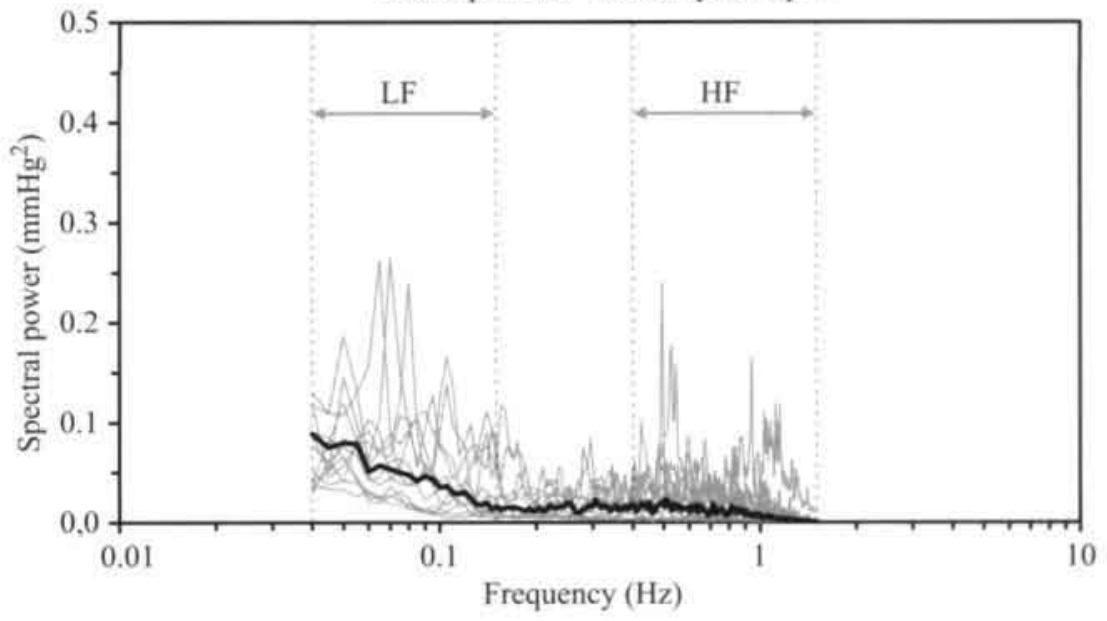

Figure 3. Change in spectral power for SBP series after atropine administration. The individual spectral density curves and the (group) median spectral density curve of SBP series are shown for the control state (top) and after atropine (bottom). The spectral power $\left(\mathrm{mmHg}^{2}\right)$ is distributed as a function of frequency $(\mathrm{Hz})$. The $x$ axis is logarithmic scaled in order to visualize the LF band better. The spectral density curves of the individual subjects are presented as thin lines and the median value as a bold line. The vertical lines indicate the boundaries of the low frequency band (LF: $0.04-0.15 \mathrm{~Hz}$ ) and high frequency band (HF: 0.4$1.5 \mathrm{~Hz}$ ). In the LF band individual peaks can be observed corresponding with baroreceptor reflex activity. The slight increase of spectral power in the HF band corresponds to respiratory activity. In contrast to the reduction in variability in $\mathrm{R}-\mathrm{R}$ interval series, total systolic blood variability is preserved after atropine, and is even $25 \%$ higher after atropine. 
Table 4: Transfer function analysis

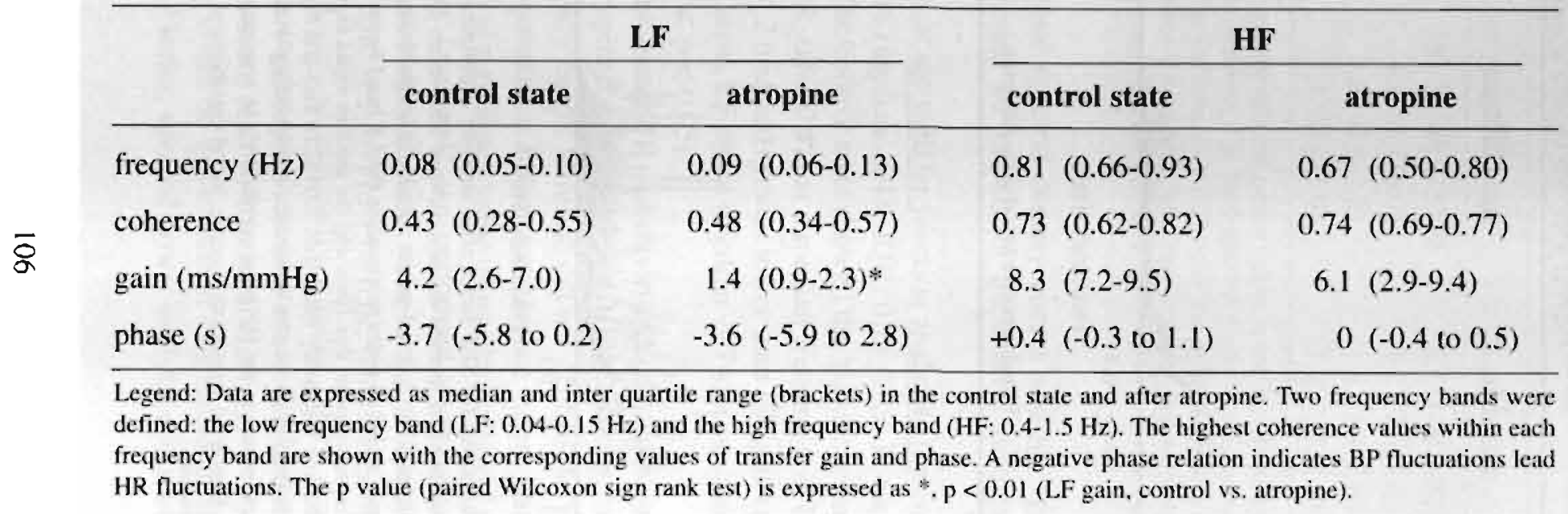




\section{Discussion}

This study demonstrates clearly that vagal tone is present in the very first days after birth in preterm infants. Administration of atropine resulted in a significant increase in HR without altering SBP. Atropine profoundly decreased total variability of the R-R interval series, which was associated with a slight increase in total variability of SBP series. Remarkably, for R-R interval series, the decrease in LF power was larger than the decrease in HF power. The transfer function analysis showed a significant decrease in LF transfer gain (BR sensitivity) after atropine.

Endotracheal intubation is a commonly performed procedure in neonatal intensive care, and atropine is used as premedication to facilitate intubation of neonates ${ }^{24}$. Atropine, a muscarinic receptor antagonist, exerts its influence on postganglionic muscarinic receptors of the sino-atrial node and atrioventricular node of the heart. Binding of acetylcholine to muscarinic receptors leads to a rapid (within $100 \mathrm{~ms}$ ) membrane hyperpolarization ${ }^{25}$. In addition, the effect of vagal impulse is brief because acetylcholine is rapidly hydrolyzed ${ }^{25}$. Both characteristics of acetylcholine enable the parasympathetic system to be a fast-acting system. In contrast, cardiac responses to sympathetic stimulation arise relatively slowly (latency of 2-5 s) and dissipate over a longer period of time ${ }^{25}$. It is assumed that LF fluctuations of R-R interval and SBP are attributed to the $B R$ and mediated by sympathetic as well as parasympathetic nerve activity. HF fluctuations of R-R interval and SBP are associated with respiratory activity and HF R-R interval fluctuations are mainly mediated through the (fast-acting) parasympathetic system $1,2,7,15,22,23,25$.

$H R$ and BP variability: The observations that atropine increases $H R$ and decreases its total variability are in agreement with findings from animal data as well as human data $26-32$. The observation of unchanged SBP with increased SBP variability is confirmed by previous studies ${ }^{28,29}$. Remarkably, we observed that at LF, the profound decrease in variability of the R-R interval series was not associated with an increase in variability of the SBP series. Apparently, at LF, the (slow acting) sympathetic system is capable of adjusting SBP fluctuations. In contrast, the increase of SBP variability in the HF band after atropine suggests a loss of a (fast acting) buffering system as a result of atropine. In preterm infants with respiratory distress syndrome and patency of the ductus arteriosus, it is possible that the increase in $\mathrm{HR}$ after atropine induces an increase in transductal shunting, which is known to be associated with an increase in HF BP variability ${ }^{33}$. However, in the present study, none of the subjects had ductal patency during the measurements.

The extreme thorax movements in patients who have severe respiratory distress and are 
breathing spontaneously may directly and mechanically exert profound oscillations in $\mathrm{BP}$, which cannot be buffered completely by beat-to-beat variation in R-R intervals ${ }^{34}$. Furthermore, one could speculate that in these patients, the respiratory fluctuations are faster than the frequency response of the heart to fluctuations in parasympathetic activity ${ }^{35}$. Respiration may produce, via the BR, increasing and decreasing concentrations of acetylcholine at the sinus node. The response frequency of the sinus node, however, may be too slow to follow with acceleration and deceleration to these alternating concentrations of acetylcholine. This frequency-limiting step of the parasympathetic system might be related to the cellular properties of an immature parasympathetic nervous system or immature cardiac target cells. Parasympathetic innervation and acetylcholine esterase activity in the neonatal cardiac conduction system is only moderately present at birth, and a process of maturation presumably takes place later in life ${ }^{36}$. This could explain why atropine showed less effect on the HF respiratory-associated $\mathrm{R}-\mathrm{R}$ interval fluctuations. Three other explanations are possible for the small effect on HF fluctuations in R-R interval series. First, the small effect of atropine on $\mathrm{HF}$ could be explained by the fact that $\mathrm{HF}$ is relatively low in sick preterm infants. Second, the study is not designed as a classical pharmacological blockade study. The muscarinic receptor blockade is not validated for total blockade with agonists. However, we found in all subjects a marked HR increase after atropine and thus it seems unlikely that the dose was too small. Notably, we observed no initial 'paradoxal' vagomimetic period, resulting from a low dose of atropine that can be seen in adults before the vagolytic effect ${ }^{32}$. Third, the infants are in respiratory distress. The extreme thorax movements probably lead to direct stretching of the atria, not related to $\mathrm{BR}$, resulting in respiratory-associated $\mathrm{R}-\mathrm{R}$ interval fluctuations ${ }^{37}$.

One of the interesting aspects of our study is the observation that atropine predominantly decreases the LF fluctuations and not the respiratory-associated HF fluctuations of the R-R interval series. These findings are in contrast with animal or human adult data. In adult rats, atropine increased $\mathrm{HR}$ and decreased the spectral HF peak ${ }^{29,31}$. Similar results have been obtained in human adults, in which atropine increased HR and decreased HF spectral power ${ }^{30,32,38}$. During sympathetic stimulation, variability of the R-R interval series showed virtually no spectral power in the HF band after atropine ${ }^{30}$. In a study of thermoregulatory blood flow fluctuations in human adults, atropine resulted in a significant increase in HR. However, parasympathetic blockade caused a great reduction in power in all frequency bands in the HR spectrum, with remaining power in the LF band ${ }^{39}$. Studies in healthy volunteers under controlled conditions ( $\beta$-adrenoceptor blockade, metronomic breathing) show that the respiratoryassociated fluctuations are more sensitive to slight changes in vagal activity than to tonic control ${ }^{32}$. To explain these features, it can be hypothesized that difference in the 
shift of HR and respiratory-associated fluctuations are controlled by independent actions of the two primary medullary source nuclei of the vagus: the nucleus ambiguus and the dorsal motor nucleus. This hypothesis could fit with a phylogenetic theory named the polyvagal theory ${ }^{40,41}$. Although vagal pathways from both nuclei terminate on the sinoatrial node, it is argued that the fibers that originate in the nucleus ambiguous are uniquely responsible for the phasic HR control, whereas the dorsal motor nucleus controls the neurogenic tonic $\mathrm{HR}^{4}$. It is possible that the different vagal nuclei behave differently when exposed to atropine. Furthermore, it is likely that in preterm infants, the vagal system is not fully matured, has different myelination of the vagal nerve, or lacks the interconnections between the two vagal nuclei to ensure the (adult) phasic cardiorespiratory control ${ }^{40,42}$.

The observation of a predominant effect on decreasing the LF spectral power of the R$\mathrm{R}$ interval series after atropine indicates that the $\mathrm{BR}$ in preterm infants is under significant parasympathetic control. These vagal responses might reflect different physiology in the preterm infant, as discussed above. Several other explanations can be given for a predominant decrease in LF power of the R-R interval series. First, in addition to the dominant cardiac cholinergic (muscarinic) M2 receptor found in the adult heart, $\mathrm{Ml}$ subtypes may be found in the neonatal heart ${ }^{43}$. These neonatal receptors may serve different physiologic responses to acetylcholine stimulation ${ }^{44}$. Second, parasympathetic activity can modulate sympathetic effects by inhibiting norepinephrine release and modulating the sympathetic system ${ }^{45}$. Likewise, blockade of the parasympathetic system might have an effect on sympathetic activity. Unknown is to what extent these interactions and processes are maturated in preterm infants. Third, because breath amplitude modulation is associated with (very) LF fluctuations in R-R interval series, the decrease of the LF component might be caused by a more stable respiration pattern after atropine administration 46 .

In adults, the ratio low-to-high spectral power in R-R interval series (LF/HF ratio) is assumed to reflect the 'sympathovagal' balance ${ }^{2,15}$. LF depends on sympathetic and parasympathetic activity, and HF is mediated by parasympathetic activity. This ratio has never been validated in neonates. Remarkably, we observed a significant decrease in the $\mathrm{LF} / \mathrm{HF}$ ratio of the R-R interval series following atropine. Considering the predominant effect on decreasing the LF component of the R-R interval series after atropine and possible different physiology of cardiovascular regulation as discussed above, the LF/HF ratio might not be applicable in preterm infants in the transitory phase after birth. 
Transfer function analysis and BR: The control state in our study showed the highest coherence values within the LF band at approximately $0.08 \mathrm{~Hz}$, comparable with the natural frequency of the BR. In addition, we found in the control state a LF transfer gain (or BR sensitivity) of approximately $4 \mathrm{~ms} / \mathrm{mmHg}$. Of the total study group, 9 subjects showed a clear negative phase relation between SBP fluctuations and R-R interval changes, indicating a BR mediated reflex: LF fluctuations in SBP lead R-R interval changes. In 3 subjects a slightly positive phase relation between SBP fluctuations and $\mathrm{R}-\mathrm{R}$ interval changes was observed, possibly suggesting a feed-forward mechanism: LF changes in R-R interval lead fluctuations in SBP. No data are available in neonates on whether such a feed-forward mechanism exists. Alternatively, the circumstances during which the data are acquired (very preterm infants in respiratory distress, high arousal state) possibly limited the transfer function analysis. However, all subjects of the subgroup analysis with coherence values $>0.5$ (assumed to be statistically more reliable) showed a negative phase relation, indicating a BR mechanism: LF fluctuations in SBP lead R-R interval changes by approximately $4 \mathrm{~s}$. These observations are in agreement with our previous report on BR functioning in respiratory stable preterm 6 . We noticed after atropine administration a significant decrease in LF gain. LF gain is decreased because of the predominant effect of atropine on decreasing LF variability of the R-R interval series. LF phase relation did not change after atropine. This indicates that atropine changes the BR sensitivity but does not completely diminish the BR in preterm infants presumably because of sympathetic regulation. This aspect of decreased sensitivity is in agreement with human adult data in which atropine leads to a flattening of the cardiac baroreflex slope ${ }^{47}$. In case of HR frequencies of $2.5 \mathrm{~Hz}$ and respiratory frequencies of approximately $1 \mathrm{~Hz}$, taking into account the closed-loop properties of the $B R$, interpretation of the HF phase shift is limited. At HF, we observed a median phase between SBP and R-R interval fluctuations was close to $0 \mathrm{~s}$ with only small IQRs $(-0.3$ to $+0.5 \mathrm{~s}$ ), indicating that $\mathrm{SBP}$ and $\mathrm{R}-\mathrm{R}$ interval series are fluctuating together and probably not related to $\mathrm{BR}$ activity ${ }^{29}$.

Methodological considerations: A limitation of the study group is its heterogeneity regarding the underlying pathology of the preterm infants. In contrast to our earlier study, we found in the present study lower coherence values of the LF transfer function analysis. The lower coherence values might be caused by acquisition 'unselecting' data during the study. In contrast to our previous study, we analyzed much longer periods without taking the (quiet) sleep state into account. In addition, the character of the "acute" intervention (15 min of control state - atropine administration - 15 min after atropine but before endotracheal intubation) precludes selecting such periods. For the same reason we opted for a predefined HF band. 


\section{Conclusion}

The present study shows a significant vagal tone is already present in the transitory phase after birth. Atropine increases $H R$ and decreases variability of R-R interval series. In contrast to R-R interval series, variability of SBP series is preserved. Remarkably, atropine predominantly decreases the LF fluctuations of R-R interval series. As a consequence, atropine decreases the LF transfer gain or BR sensitivity. These data show that atropine modulates $\mathrm{HR}$ and $\mathrm{BP}$ variability, suggesting that BR-mediated parasympathetic control of HR is of significance for cardiovascular control in the early postnatal period of distressed preterm infants. 


\section{References}

1. Akselrod S, Gordon D, Madwed JB, Snidman NC, Shannon DC, Cohen RJ 1985 Hemodynamic regulation: investigation by spectral analysis. Am J Physiol 249:H867-H875

2. Malliani A, Pagani M, Lombardi F, Cerutti S 1991 Clinical and experimental evaluation of sympatho-vagal interaction: power spectral analysis of heart rate and arterial pressure variabilities In: Gilmore JP, Zucker IH (eds) Reflex control of the circulation. CRC Press, Boca Raton, FL, pp 1194

3. Waldman S, Krauss AN, Auld PAM 1979 Baroreceptors in preterm infants: their relationship to maturity and disease. Develop Med Child Neurol 21:714-722

4. Picton-Warlow CG, Mayer FE 1970 Cardiovascular responses to postural changes in the neonate. Arch Dis Child 45:354-359

5. Gournay V, Drouin E, Rozé JC 2002 Development of baroreflex control of heart rate in preterm and full term infants. Arch Dis Child Fetal Neonatal Ed 86:F151-154

6. Andriessen P, Koolen AMP, Berendsen RCM, Wijn PFF, ten Broeke EDM, Oei SG, Blanco CE 2003 Cardiovascular fluctuations and transfer function analysis in stable preterm infants. Pediatr Res 53:89-97

7. Shinebourne EA, Vapaavuori EK, Williams RL, Heymann MA, Rudolph AM 1972 Development of baroreflex activity in unanesthetized fetal and neonatal lambs. Circ Res 31:710-718

8. Dawes GS Johnston BM, Walker DW 1980 Relationship of arterial pressure and heart rate in fetal, newborn and adult sheep. J Physiol 309:405-417

9. Altimiras J. Crossley II DA 2000 Control of blood pressure mediated by baroreflex changes of heart rate in the chicken embryo (Gallus gallus). Am J Physiol 278:R980-R986

10. Segar JL 1997 Ontogeny of the arterial and cardiopulmonary baroreflex during fetal and postnatal life. Am J Physiol 273:R457-R471

11. Assali NS, Brinkman CR 3rd, Woods JR, Dandavino A, Nuwayhid B 1977 Development of neurohumoral control of fetal, neonatal, and adult cardiovascular functions. Am J Obstet Gynecol 129:748-759

12. Nuwayhid B, Brinkman CR 3rd, Su C, Bevan JA. Assali NS 1975 Development of autonomic control of fetal circulation. Am J Physiol 228:337-344

13. Minoura S, Gilbert RD 1987 Postnatal changes of cardiac function in lambs: effects of ganglionic block and afterload. J Dev Physiol 9:123-135

14. Kloosterman GJ 1970 On intrauterine growth. Int J Gynaec Obst 8:895-912

15. Task Force of the European Society of Cardiology and the North American Society of pacing and electrophysiology 1996 Heart rate variability: standards of measurement, physiological interpretation, and clinical use. Circulation 93:1043-1065

16. van Ravenswaay-Arts CMA, Hopman JCM, Kollée LAW. Stoelinga GBA, van Geijn HP 1994 Spectral analysis of heart rate variability in spontaneously breathing very preterm infants. Acta Paediatr 83:473-480

17. Spassov L. Curzi-Dascalova L, Clairambault J, Kauffmann F, Eiselt M, Medigue C, Peirano P 1994 Heart rate and heart rate variability during sleep in small-for-gestational age newborns. Pediatr Research 35:500-505

18. Chatow U, Davidson S, Reichman BL, Akselrod S 1995 Development and maturation of the autonomic nervous system in premature and full-term infants using spectral analysis of heart rate fluctuations. Pediatr Res 37:294-302

19. Mazursky JE, Birkett CL, Bedell KA, Ben-Haim SA, Segar JL 1998 Development of baroreflex influences on heart rate variability in preterm infants. Early Hum Dev 53:37-52 
20. Sahni R, Schulze KF, Kashyap S, Ohira-Kist K, Fifer WP, Myers MM 1999 Postural differences in cardiac dynamics during quiet and active sleep in low birth infants. Acta Paediatr 88:1396-1401

21. Janssen BJA, Leenders PJA, Smits JFM 2000 Short-term and long-term blood pressure and heart rate variability in the mouse. Am J Physiol 278:R215-R225

22. Head GA, Lukoshkova EV, Burke SL, Malpas SC, Lambert EA, Janssen BJA 2001 Comparing spectral and invasive estimates of baroreflex gain. IEEE Eng Med Biol Mag 20(2):43-52

23. Robbe HW. Mulder LJM, Rüddel H. Langewitz WA. Veldman JBP, Mulder G 1987 Assessment of baroreceptor reflex sensitivity by means of spectral analysis. Hypertension 10:538-543

24. Barrington KJ, Finer NN, Etches PC 1989 Succinylcholine and atropine for premedication of the newborn infant before nasotracheal intubation: A randomized, controlled trial. Crit Care Med 17:1293-1296

25. Salata JJ, Zipes DP 1991 Autonomic nervous system control of heart rate and atrioventricular nodal conduction. In: Gilmore JP, Zucker IH (eds) Reflex control of the circulation. CRC Press, Boca Raton, FL, pp 67-101

26. Woods JR, Dandavino A, Murayama K, Brinkman CR, Assali NS 1977 Autonomic control of cardiovascular functions during neonatal development and in adult sheep. Circ Res 40:401-407

27. Goldberg JM, Moberg GP 1985 Autonomic control of heart rate in the neonatal thesus monkey. J Med Primatol 14:19-27

28. Ferrari AU, Daffonchio A, Albergati F, Mancia G 1987 Inverse relationship between heart rate and blood pressure variabilities in rats. Hypertension 10:533-537

29. Cerutti C, Gustin MP, Paultre CZ, Lo M, Julien C, Vincent M, Sassard J 1991 Autonomic nervous system and cardiovascular variability in rats: a spectral analysis approach. Am J Physiol 261:H1292H1299

30. Saul JPh, Berger RD, Albrecht P, Stein SP, Hui Chen M, Cohen RJ 1991 Transfer function analysis of the circulation: unique insights into cardiovascular regulation. Am J Physiol 261:H1231-H1245

31. Japundzic N. Grichois ML, Zitoun P. Laude D, Elghozi JL 1990 Spectral analysis of blood pressure and heart rate in conscious rats: effects of autonomic blockers. J Auton Nerv Syst 30:91-100

32. Medigué C, Girard A, Laude D, Monti A, Wargon M, Elghozi JL 2001 Relationship between pulse interval and respiratory sinus arrhythmia; a time- and frequency-domain analysis of the effects of atropine. Eur J Physiol 441:650-655

33. Beuchée A. Pladys P, Senhadji L, Bétrémieux P, Carré F 2003 Beat-to-beat blood pressure variability and patent ductus arteriosus in ventilated, premature infants. Eur J Physiol 446:154-160

34. Metsälä T. Siimes A. Antila K, Välimäki I 1993 Association of breathing movements to the variability of heart rate and blood pressure in foetal lambs. Acta Physiol Scand 147:213-219

35. Stauss HM, Persson PB, Johnson AK, Kregel KC 1997 Frequency-response characteristics of autonomic nervous system function in conscious rats. Am J Physiol 273:H786-795

36. Chow LT, Chow SS, Anderson RH, Gosling JA 1995 The innervation of the human myocardium at birth. J Anat 187:107-114

37. Bernardi L, Keller F, Sanders M, Reddy PS, Griffith B. Meno F, Pinsky MR 1989 Respiratory sinus arrhythmia in the denervated human heart. J Apply Physiol 67:1447-1455

38. Toska K, Eriksen M 1993 Respiration-synchronous fluctuations in stroke volume, heart rate and arterial pressure in humans. J of Physiol 472:501-512

39. Lossius K. Eriksen M. Walloe L 1994 Thermoregulatory fluctuations in heart rate and blood pressure in humans: effect of cooling and parasympathetic blockade. J Auton Nerv Syst 47:245-254

40. Taylor EW 1994 The evolution of efferent vagal control of the heart in vertebrates. Cardioscience 5:173-182

41. Porges SW 1995 Orienting in a defensive world: Mammalian modifications of our evolutionary heritage. A polyvagal theory. Psychophysiology 32:301-318 
42. Sachis PN, Armstrong DL, Becker LE, Bryan AC 1982 Myelination of the human vagus nerve from 24 weeks postconceptional age to adolescence. J Neuropathol Exp Neurol 41:466:472

43. Pickoff AS 1998 Developmental electrophysiology in the fetus and neonate. In: Polin RA, Fox WW (eds) Fetal and neonatal physiology. WB Saunders Company, Philadelphia, pp 891-913

44. Danilo P Jr, Rosen MR, Hordof AJ 1978 Effects of acetylcholine on the ventricular specialized conducting system of neonatal and adult dogs. Circ Res 43:777-784

45. Randall WC, Randall DC. Ardell JL 1991 Autonomic regulation of myocardial contractility. In: Gilmore JP, Zucker IH (eds) Reflex control of the circulation. CRC Press, Boca Raton, FL. pp 39-65

46. Dykes FD, Ahmann PA, Baldzer K. Carrigan TA, Kitney R, Giddens DP 1986 Breath amplitude modulation of heart rate variability in normal full term neonates. Pediatr Res 20:301-308

47. Parlow J, Viale JP, Annat G, Hughson R, Quintin L 1995 Spontaneous cardiac baroreflex in humans: comparison with drug-induced responses. Hypertension 25:1058-1068 


\section{Chapter 8}

\section{Baroreceptor reflex sensitivity in neonates: the effect of gestational age}

Peter Andriessen, Chris H.L. Peters, Barbara Vermeulen, Sidarto Bambang Oetomo, Pieter F.F. Wijn, Carlos E. Blanco

submitted 


\section{Abstract}

The effect of gestational age on baroreceptor reflex sensitivity (BRS) was studied in 32 infants (postconceptional age, PCA, range: 28-42 wk) during 3-min periods of quiet sleep in the first 10 days after birth. Cross-spectral power analysis between systolic blood pressure (SBP) and R-R fluctuations, using fast Fourier transform, was estimated in a low frequency (LF, 0.04-0.15 Hz), high frequency $(\mathrm{HF}$, individualized between $\mathrm{p}$ 10 and p-90 value of respiratory frequency), and total frequency band $(0.04-1.5 \mathrm{~Hz})$. BRS was estimated using transfer function analysis (transfer gain or BRS, $\mathrm{ms} / \mathrm{mmHg}$; phase, s) between LF fluctuations of SBP and R-R.

With PCA the mean R-R interval and LF-, HF-, and total spectral power of R-R interval series increased significantly. PCA significantly correlated with LF transfer gain $(B R S=1.1 \cdot P C A-30 \mathrm{~ms} / \mathrm{mmHg}, \mathrm{r}=0.80, \mathrm{p}<0.01)$. Median BRS was 4.6 (IQR, 3.15.4), 7.5 (IQR, 5.2-10.1) and 15.0 (IQR, 11.8-19.7) $\mathrm{ms} / \mathrm{mmHg}$ for infants with a PCA between 28-32 wk ( $\mathrm{n}=16), 32-37 \mathrm{wk}(\mathrm{n}=10)$, and 37-42 wk $(\mathrm{n}=6)$, respectively. For LF transfer phase no differences between PCA groups were found: SBP fluctuations lead R-R interval changes by approximately $3 \mathrm{~s}$. BRS correlated significantly with LF-, HF-, and total spectral power values of the R-R interval series. By contrast, BRS did not show a significant correlation with the spectral values of SBP series.

This study demonstrates that BRS is limited in preterm infants, but increases with PCA. The increase in BRS with PCA is the result of maturation of the parasympathetic system.

\section{Abbreviations}

BP blood pressure

BR baroreceptor reflex

BRS baroreceptor reflex sensitivity

HF high frequency (frequency between p- 10 and p-90 of the individual respiratory frequency)

HR heart rate

IQR interquartile range (P25 - P75)

LF low frequency $(0.04-0.15 \mathrm{~Hz})$

PCA postconceptional age

$\mathrm{R}-\mathrm{R} \quad \mathrm{R}-\mathrm{R}$ interval length

SBP systolic blood pressure 


\section{Introduction}

The baroreceptor reflex (BR) is the most important regulatory mechanism in the shortterm control of heart rate (HR) and blood pressure (BP). The BR buffers sudden changes in systemic BP by adapting HR and peripheral vascular resistance. HR responses are mediated by both parasympathetic and sympathetic efferent nerve activity, whereas vascular resistance is adapted by sympathetic nerves only. Because vascular resistance is difficult to measure autonomic changes related to BR control are usually studied by evaluating HR and BP changes only.

Two parameters are often determined to estimate BR function ${ }^{1}$. First, the operating point of the reflex: at which level does the reflex responds most effectively to changes in arterial pressure. Second, the gain or BR sensitivity (BRS): the magnitude of the reflex response per unit of arterial $\mathrm{BP}$ deviation from the operating point. The BRS is classically defined as the slope of the linear part of the (sigmoid) BP-HR relationship (HR or R-R interval change per unit of BP change) plotted during the pressure rise (pressor response) or decrease (depressor response), induced by the administration of epinephrine or sodium nitroprusside, respectively ${ }^{2-4}$.

The pharmacological method of altering the control of the cardiovascular system, however, is limited in neonates for medical ethical reasons. Spectral analysis offers the opportunity to decompose spontaneously occurring fluctuations in BP and R-R interval series into a power spectrum, and to relate the character of the fluctuations to physiological processes. Low frequent (LF) BP and R-R interval fluctuations at a frequency of approximately $0.1 \mathrm{~Hz}$ are ascribed to the BR activity and high frequent (HF) fluctuations are associated with respiratory activity and vagal modulation ${ }^{5}$. Crossspectral (transfer function) analysis between BP and R-R interval fluctuations in the LF band $(0.04-0.15 \mathrm{~Hz})$ has shown to be an estimate of the BRS ${ }^{6-9}$.

Previously we showed the feasibility to use cross-spectral analysis to estimate BRS from spontaneous BP and HR fluctuations in stable preterm infants ${ }^{10}$. We found a BRS of approximately $4 \mathrm{~ms} / \mathrm{mmHg}$ in stable preterm infants (gestational age, 28-32 wk) in the first week of life. This value of the BRS is consistent with results of other investigators, although they used a different (time sequence) technique in assessing the BRS 11,12. Furthermore these authors showed higher BRS values in term babies (approximately $10 \mathrm{~ms} / \mathrm{mmHg}$ ), indicating a maturational effect of gestation on BRS. We did not show a correlation between gestational age and BRS, presumably because of the small range in $\mathrm{GA}$ and small size of the group ${ }^{10}$. For this reason we want to extent the range of postconceptional age (PCA, ranging from very preterm to full term infants) in order to investigate the influence of age on BRS.

The objectives of the study were to determine 1) the effect of PCA on spectral power of 
R-R interval and SBP series; 2) the effect of PCA on BRS investigated by cross-spectral power analysis of spontaneously occurring fluctuations in SBP and R-R interval series (transfer function analysis: coherence, LF gain or BRS, phase relation); and 3) to study the relationship between BRS and spectral power analysis of R-R interval and SBP series.

\section{Methods}

Subjects: The study group consisted of 32 clinically stable infants (gestational age, 32.1 $\pm 3.7 \mathrm{wk}$; birth weight, $1855+808 \mathrm{~g}$ ) and were studied once in the immediate postintensive care phase between 1 and 10 days of postnatal life ( $4.1 \pm 2.3$ days after birth). After correction for the postnatal age of measurement, the study group consisted of 16 preterm infants with a PCA between 28-32 wk, 10 preterm infants with a PCA between 32-37 wk and 6 term infants with a PCA between 37 and 42 wk. Table 1 shows the clinical characteristics of the study group. The primary diagnosis for the preterm infants were respiratory distress syndrome $(n=23)$, apnea $(n=2)$ and hyper viscosity syndrome $(\mathrm{n}=1)$. For term babies, the primary diagnosis were exchange transfusion for

Table 1: Clinical characteristics of the study population

\begin{tabular}{|c|c|c|c|}
\hline 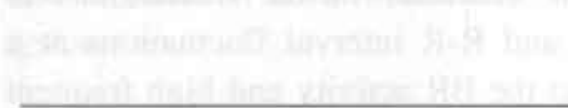 & $\begin{array}{c}\text { PCA 28-32 } \\
(n=16)\end{array}$ & $\begin{array}{c}\text { PCA 32-37 } \\
(n=10)\end{array}$ & $\begin{array}{c}\text { PCA } 37-42 \\
(n=6)\end{array}$ \\
\hline Gestational age (wk) & $29.3 \pm 1.4$ & $32.9 \pm 1.2$ & $38.4 \pm 1.7$ \\
\hline Birth weight (g) & $1333 \pm 277$ & $1872 \pm 468$ & $3220 \pm 565$ \\
\hline Postnatal day of measurements & $4.7 \pm 2.7$ & $3.3 \pm 1.5$ & $3.8 \pm 2.1$ \\
\hline Corrected PCA & $29.9 \pm 1.2$ & $33.4 \pm 1.0$ & $39.0 \pm 1.7$ \\
\hline Umbilical arterial $\mathrm{pH}$ & $7.30 \pm 0.06$ & $7.20 \pm 0.07$ & $7.22 \pm 0.08$ \\
\hline 5-min Apgar score (median, IQR) & $8(7-9)$ & $8(7-9)$ & $8(6-9)$ \\
\hline Antenatal corticoids (\%) & 63 & 40 & 0 \\
\hline Surfactant $(\%)$ & 38 & 60 & 0 \\
\hline Prior mechanical ventilation (d) & $2.0 \pm 2.0$ & $1.2 \pm 1.4$ & $2.6 \pm 2.7$ \\
\hline $\begin{array}{l}\text { Prior patent ductus arteriosus } \\
\text { closed by indomethacine }(\%)\end{array}$ & 19 & 0 & 0 \\
\hline Caffeine $(\%)$ & 88 & 30 & 0 \\
\hline
\end{tabular}


hyperbilirubinemia $(\mathrm{n}=1)$, transient tachypnoe of the newborn or pneumonia $(\mathrm{n}=3)$ and idiopathic neonatal convulsions $(n=2)$. All infants were appropriate-for-gestational age, according to the Dutch growth charts ${ }^{13}$. Arterial catheters were neither inserted nor remained longer in place because of the study. All infants were breathing room air spontaneously. None had echoencephalographic evidence of cerebral hemorrhage or parenchymal lesions. Echocardiography revealed no structural abnormalities. All infants were judged as cardiovascular stable without need of cardiotropic drugs (dopamine, dobutamine) or volume expanders at the time of the study. There were no signs or symptoms of asphyxia, respiratory distress, sepsis, or patency of the ductus arteriosus at the time of measurements. The two babies with idiopathic convulsions had normal electroencephalographic tracings at the time of measurements and were on phenobarbital therapy with plasma levels within the therapeutic range. Of the preterm infants who were on caffeine therapy, serum concentration levels were between 10 and $17 \mathrm{mg} / \mathrm{l}$. Values of electrolytes, blood gas analysis, and hematocrits were all within normal range at the time of the study. Informed consent was obtained from the parents of each infant. The local Ethics Committee approved the study.

Data acquisition: A bipolar chest lead of the surface ECG (sample frequency, $512 \mathrm{~Hz}$ ) and the transthoracic electric impedance waveforms were recorded by Hewlett Packard neonatal monitor type Merlin (Waltham, MA, USA). Arterial BP (sample frequency, $256 \mathrm{~Hz}$ ) was measured invasively through a 4 French catheter in the aortic position $(n=26)$ or in the right radial artery $(n=6)$, used for routine monitoring of vital functions and intensive care management. A $0.5 \mathrm{ml} / \mathrm{h}$ infusion of heparinized physiological saline solution was continuously flushed through the catheter. Recordings were made in the prone position for 1-2 h. Data segments were selected subsequently during periods of regular breathing and spontaneous sleep with closed eyes and without gross body movements (quiet sleep state) ${ }^{14}$. Data analysis was performed on 192-s-long segments. Because preterm infants have an immature and irregular respiratory drive, 3-min segments were chosen as a compromise between the demands of sufficient duration and signal stability. The data selection during the quiet sleep state resulted in R-R interval artifact free segments.

Data analysis: With respect to details of the spectral analysis we refer to a previous report ${ }^{10}$. Briefly, R waves were detected from the ECG and an unevenly spaced R-R interval sequence was created. This sequence was resampled at $4 \mathrm{~Hz}$ in order to obtain an equidistantly spaced time series ${ }^{15}$. The mean R-R interval was calculated and subtracted from all data points to remove the direct current component. SBP was identified from peak detection of the BP signal, resulting in an unevenly spaced 
"systogram". The "systogram" was converted into an equidistantly spaced time series using the same resampling method as used for the R-R interval time series. Each $192-$ s-long segment of preprocessed evenly spaced $R-R$ interval and SBP series was subdivided in 5 half-overlapping 64-s (256 points) segments. To reduce the effects of spectral leakage each segment was multiplied with a triangular Parzen window ${ }^{16}$. FFT was used to compute the auto- and cross-spectral density functions for each 256 points segment. A mean power spectrum was derived from the 5 spectral density functions and the spectral power values in the ranges of interest were calculated. Using 64-s segments (resulting in a spectral resolution of $0.016 \mathrm{~Hz}$ ) and 5 half-overlapping segments spectral power estimation above $0.04 \mathrm{~Hz}$ is considered accurate. The data acquisition and analysis software package was developed at the department of Clinical Physics of our hospital in collaboration with the department of Physics of the Eindhoven University of Technology, The Netherlands.

Two frequency bands were defined, as indicated in Figure 1. The low frequency band, reflecting BR activity, was defined between $0.04-0.15 \mathrm{~Hz}$. Because the $\mathrm{HF}$ band primarily contains the reflection of respiratory associated parasympathetic activity, the $\mathrm{HF}$ band was individualized for each subject depending on his or her respiratory drive as described earlier ${ }^{10}$. The respiratory rate was estimated by peak detection of the thoracic waveforms resulting in a mean value and a bandwidth between the $10^{\text {th }}$ and $90^{\text {th }}$ centiles of the breath-by-breath frequency. The frequency range between these centiles was used to identify each subject's individual HF band. The upper spectral limit of the HF band was less than half of the mean HR thereby meeting the requirements of the Nyquist critical frequency. The very low frequency band $(0-0.04 \mathrm{~Hz})$ was discarded to avoid possible contribution of slow trend artifacts. The total frequency (TF) band of interest was the range between 0.04 and $1.5 \mathrm{~Hz}$. Spectral power was calculated in each defined frequency band. The values for spectral power are presented in units of milliseconds ${ }^{2}$ (for R-R interval series) and $\mathrm{mmHg}^{2}$ (for SBP series).

Transfer function analysis: In addition to the spectral density power, the transfer function analysis (transfer gain and phase) was calculated, as previously described in detail ${ }^{9,10}$. Briefly, transfer gain reflects the degree to which the input signal (SBP) amplitude becomes manifest in the output signal ( $\mathrm{R}-\mathrm{R}$ interval) amplitude at a discrete frequency. At LF, transfer gain is an estimate for BRS ${ }^{6-9}$. The phase difference (degrees or seconds) indicates the lead or lag of one signal with respect to the other at a discrete frequency. A negative phase relation indicates SBP fluctuations lead R-R interval changes. In addition to these calculations, the coherence function was computed to assess the amount of linear coupling between SBP and R-R interval in the frequency domain. The coherence function, which ranges from 0 (no relationship) and 1 (linear 

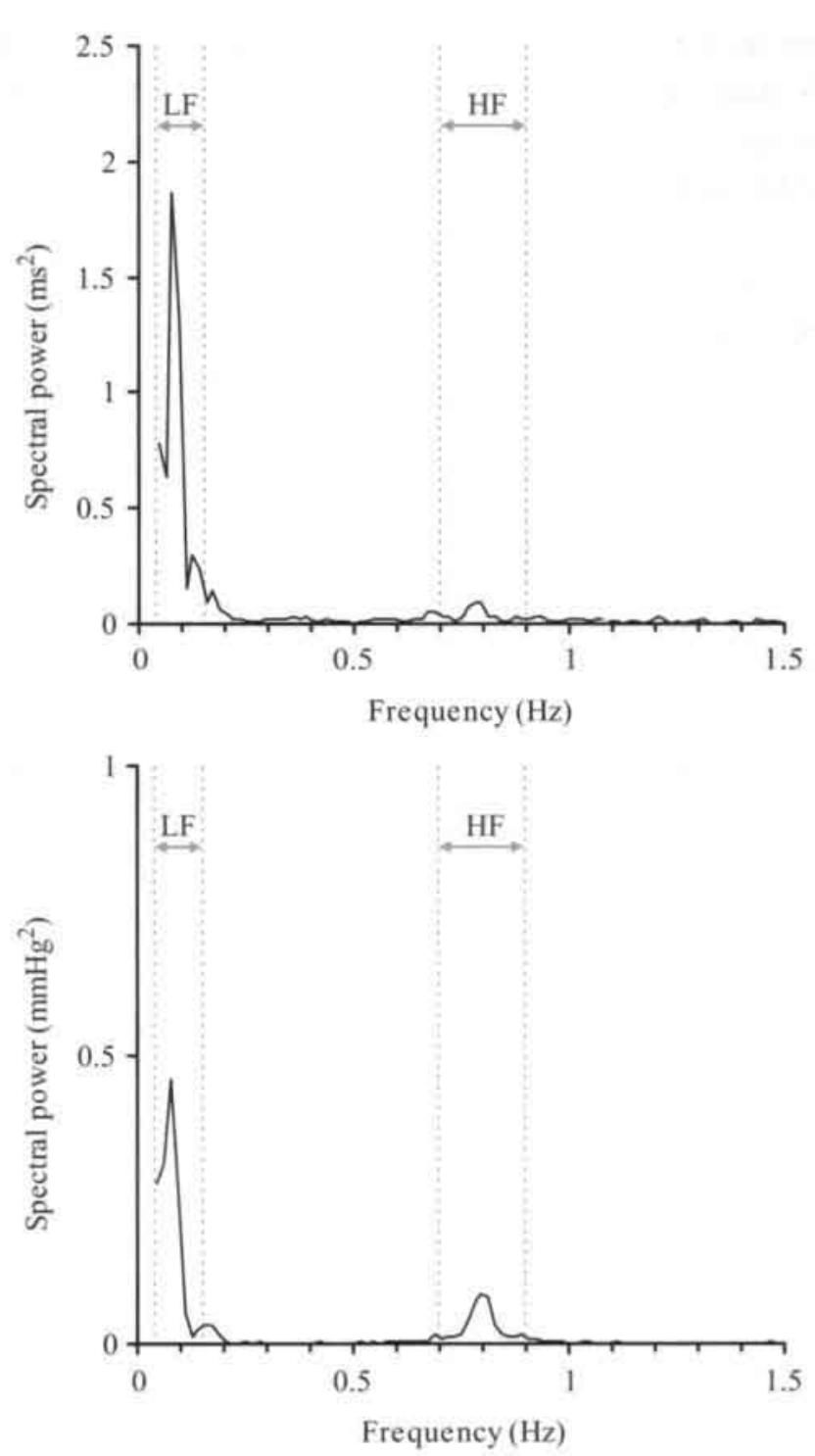

Figure 1. Spectral power curves of R-R interval and SBP series of a preterm infant measured at a PCA of 31 wk. This figure shows the spectral power curves of R-R interval (top) and SBP (bottom) series. The spectral powers ( $\mathrm{R}-\mathrm{R}$ interval series, $\mathrm{ms}^{2} ; \mathrm{SBP}$ series, $\mathrm{mmHg}^{2}$ ) are distributed as a function of frequency $(\mathrm{Hz})$. The LF band was defined between 0.04 and $0.15 \mathrm{~Hz}$ and is marked between vertical lines. The individual HF band was defined between the $10^{\text {th }}$ and $90^{\text {th }}$ centile of the individual respiratory frequency $(0.70$ and $0.90 \mathrm{~Hz}$, respectively) and is marked between vertical lines. In the LF band clear peaks in both power spectra are observed at $0.08 \mathrm{~Hz}$, assumed to be attributed to oscillation of the BR. A HF peak at 0.80 $\mathrm{Hz}$ is clearly visible in the SBP power spectrum and corresponds with the mean respiratory rate of the subject. In the R-R interval power spectrum a modest HF peak is visible. 
relationship) was used to assess the statistically reliability of the transfer function at each frequency. Only data in which the coherence was above 0.5 were used in the estimation of the transfer functions ${ }^{6-7}$. Transfer gain and phase were assessed in each frequency band at the frequency of highest coherence value ${ }^{10}$.

Statistical analysis: Data with a normal distribution are expressed as a mean $\pm \mathrm{SD}$. The spectral power values of R-R interval and SBP series and transfer function variables (frequency, coherence, gain and phase) are expressed as median and IQR. Comparisons of variables between the three PCA groups were statistically evaluated with Analysis of Variance (one-way ANOVA) and post-hoc Scheffé test for parametric data (e.g. R-R interval, SBP) and the Kruskal-Wallis $H$ test and Mann Whitney $U$ test for nonparametric data (e.g. the spectral power values and transfer function). Correlations between the variables were evaluated with the Spearmans rho rank correlation test. The influence of PCA on R-R interval, SBP and LF transfer function gain and phase was studied by linear regression analysis, using the method of least squares and ANOVA. To correct for type I error in multiple group comparisons (the post-hoc and Mann Whitney $\mathrm{U}$ test), statistical significance was accepted at $\mathrm{p}<0.017$ (Bonferroni correction, 0.05 divided by the number of pair wise comparisons, e.g. 0.05/3). For other tests statistical significance was accepted at $\mathrm{p}<0.05$.

\section{Results}

The mean and SD of HR and SBP of each subject were calculated from the 192-s-long segments, during comparable quiet sleep state. Linear regression analysis showed that $\mathrm{HR}$ and SBP were both significantly correlated with PCA $(H R=-2.0 \cdot P C A+205 \mathrm{bpm}$, $\mathrm{r}=0.61, \mathrm{p}<0.01 ; S B P=0.5 \cdot P C A+39 \mathrm{mmHg}, \mathrm{r}=0.42, \mathrm{p}<0.05)$.

An example of R-R interval and SBP power spectra of a spontaneously regularbreathing preterm subject measured at a PCA of 31 wk is shown in Figure 1. Note in the power spectra the following features: 1) a clear spectral peak centered around 0.1 $\mathrm{Hz}$ in the LF band, both in the R-R interval and SBP power spectra; 2) a clear HF spectral peak centered around $0.80 \mathrm{~Hz}$ in the SBP power spectrum and a modest HF peak is seen in the R-R interval power spectrum; and 3) the LF $(0.04-0.15 \mathrm{~Hz})$ and individual $\mathrm{HF}$ band $(0.70-0.90 \mathrm{~Hz})$ are indicated by vertical dotted lines.

Table 2 displays the spectral power parameters for the three PCA groups (28-32 wk; 32 37 wk: 37-42 wk, respectively). In general, for R-R interval series, the values of all spectral power parameters (LF-, HF-, total) were significantly higher in the term infants compared with the preterm infants. For SBP series, lower total and HF spectral values 
were observed in the term infants compared with preterm infants. Table 3 shows the correlation coefficients between PCA and the spectral power values of the R-R interval and SBP series, respectively.

Figure 2 illustrates the transfer function analysis (coherence, gain and phase) between SBP and R-R interval signals of the same preterm subject as in Figure 1. Note that high coherence values are calculated for the LF and HF band. The corresponding transfer gain and phase are indicated by dots in the figure. The LF transfer gain (in this subject $2.3 \mathrm{~ms} / \mathrm{mmHg}$ ) was used to estimate the BRS. In this example, LF SBP fluctuations lead $\mathrm{R}-\mathrm{R}$ interval changes by $1.5 \mathrm{~s}$.

Table 2: Respiratory rate and spectral power values for the R-R interval and SBP series

\begin{tabular}{lccc}
\hline & $\begin{array}{c}\text { PCA 28-32 } \\
(\mathbf{n}=\mathbf{1 6})\end{array}$ & $\begin{array}{c}\text { PCA 32-37 } \\
(\mathbf{n}=\mathbf{1 0})\end{array}$ & $\begin{array}{c}\text { PCA 37-42 } \\
(\mathbf{n}=\mathbf{6})\end{array}$ \\
\hline Respiratory rate $(\mathbf{H z})$ & $0.82 \pm 0.20$ & $0.88 \pm 0.23$ & $0.77 \pm 0.20$ \\
R-R interval series & & & \\
Heart rate (beats per min) & $142 \pm 9$ & $141 \pm 10$ & $122 \pm 12 \mathrm{~s}$, \\
LF power & $18(9-36)$ & $28(12-94)$ & $65(34-262)^{\mathrm{s}}$ \\
HF power & $1(1-2)$ & $3(1-19)$ & $24(6-45)^{\mathrm{s}}$ \\
Total power & $26(10-45)$ & $36(15-150)$ & $96(83-384)^{\mathrm{s}}$ \\
LF/HF ratio & $11(7-33)$ & $12(5-27)$ & $4(2-13)$ \\
SBP series & & & \\
SBP (mmHg) & $55 \pm 4$ & $56 \pm 4$ & $59 \pm 6$ \\
LF power & $0.9(0.4-1.6)$ & $0.6(0.4-1.4)$ & $0.4(0.1-0.9)$ \\
HF power & $0.3(0.2-0.3)$ & $0.4(0.2-0.5)$ & $0.1(0.1-0.2)$ \\
Total power & $1.2(0.8-2.2)$ & $1.3(1.0-2.4)$ & $0.7(0.3-1.1)$ \\
\hline
\end{tabular}

Spectral power analysis of the study group with a PCA between 28-32 wk; 32-37 wk; 37-42 wk, respectively. The spectral power values for the R-R interval and SBP series are presented in units of $\mathrm{ms}^{2}$ and $\mathrm{mmHg}^{2}$, respectively. The $\mathrm{LF} / \mathrm{HF}$ ratio is dimensionless. The low frequency (LF) band was defined between 0.04 and $0.15 \mathrm{~Hz}$. The high frequency (HF) band was defined as the frequency range between the $\mathrm{p}-10$ and $\mathrm{p}-90$ centile of the individual respiratory frequency. The total power was computed for the frequency band between 0.04 and $1.5 \mathrm{~Hz}$.

Parametric data are expressed as mean $\pm \mathrm{SD}$. Comparisons between groups were statistically evaluated with one-way ANOVA and the post-hoc Scheffé test. Non-parametric data are expressed as median and IQR and comparisons between groups evaluated with Kruskal-Wallis $\mathrm{H}$ test and Mann Whitney $\mathrm{U}$ test: \$: $\mathrm{p}<0.01$ (PCA $37-42$ vs. 28-32); : $\mathrm{p}<0.01$ (PCA $37-42$ vs. 32-37). 
Table 3: Correlation coefficients between PCA and spectral power values

R-R interval series:

$\begin{array}{lll}\text { LF-RR } & +0.53 & \mathrm{p}<0.01 \\ \text { HF-RR } & +0.62 & \mathrm{p}<0.01 \\ \text { Total-RR } & +0.62 & \mathrm{p}<0.01 \\ \text { LF/HF ratio } & -0.32 & \mathrm{~ns}\end{array}$

SBP series:

$\begin{array}{lll}\text { LF-SBP } & -0.21 & \text { ns } \\ \text { HF-SBP } & -0.05 & n s \\ \text { Total-SBP } & -0.16 & n s\end{array}$

Correlation coefficient between PCA and spectral power values of R-R interval and SBP series.

The total power was computed for the frequency band between 0.04 and $1.5 \mathrm{~Hz}$. The low frequency (LF) band was defined between 0.04 and $0.15 \mathrm{~Hz}$. The high frequency (HF) band was defined as the frequency range between the p-10 and p-90 centile of the individual respiratory frequency. Data are expressed as the Spearman's rho correlation coefficient with corresponding $\mathrm{p}$ value; not significant (ns).

For the total population, the median coherence value between SBP and R-R interval in the LF band was 0.65 (IQR, 0.58-0.73). The high coherence values implicate reliable estimates of the LF transfer functions parameters gain and phase. Linear regression analysis showed that LF transfer gain (BRS) significantly correlated with PCA $(B R S=$ 1.1 $P C A-30 \mathrm{~ms} / \mathrm{mmHg}, \mathrm{r}=0.80, \mathrm{p}<0.01$ ) as shown in Figure 3. LF transfer phase did not correlate with PCA. For infants with a PCA between 28-32 wk the median LF transfer gain was $4.6 \mathrm{~ms} / \mathrm{mmHg}$ (IQR, 3.1-5.4), significantly lower than infants with a PCA between 32-37 wk (median, $7.5 \mathrm{~ms} / \mathrm{mmHg}$; IQR, 5.2-10.1; $<<0.01$ ). The highest LF transfer gain was found in the term babies (median, $15.0 \mathrm{~ms} / \mathrm{mmHg}$; IQR, 11.819.7). For LF transfer phase no differences between PCA groups were found: LF SBP fluctuations lead R-R interval changes by a median of $2.6 \mathrm{~s}$ (IQR, -3.8 to -2.1 ). HF transfer gain was significantly lower than LF transfer gain and HF phase was close to $0 \mathrm{~s}$ (HF transfer data not shown). Table 4 displays the details of the LF transfer function values for each PCA group.

Table 5 shows the correlation coefficients between the LF transfer gain and the spectral power values of R-R interval and SBP series. Transfer LF gain correlated significantly with LF-, HF-, and total spectral power values of the R-R interval series. By contrast, LF transfer gain did not show a significant correlation with the spectral values of SBP series. Neither the LF transfer gain nor the phase was significantly correlated with the $\mathrm{LF} / \mathrm{HF}$ ratio. 

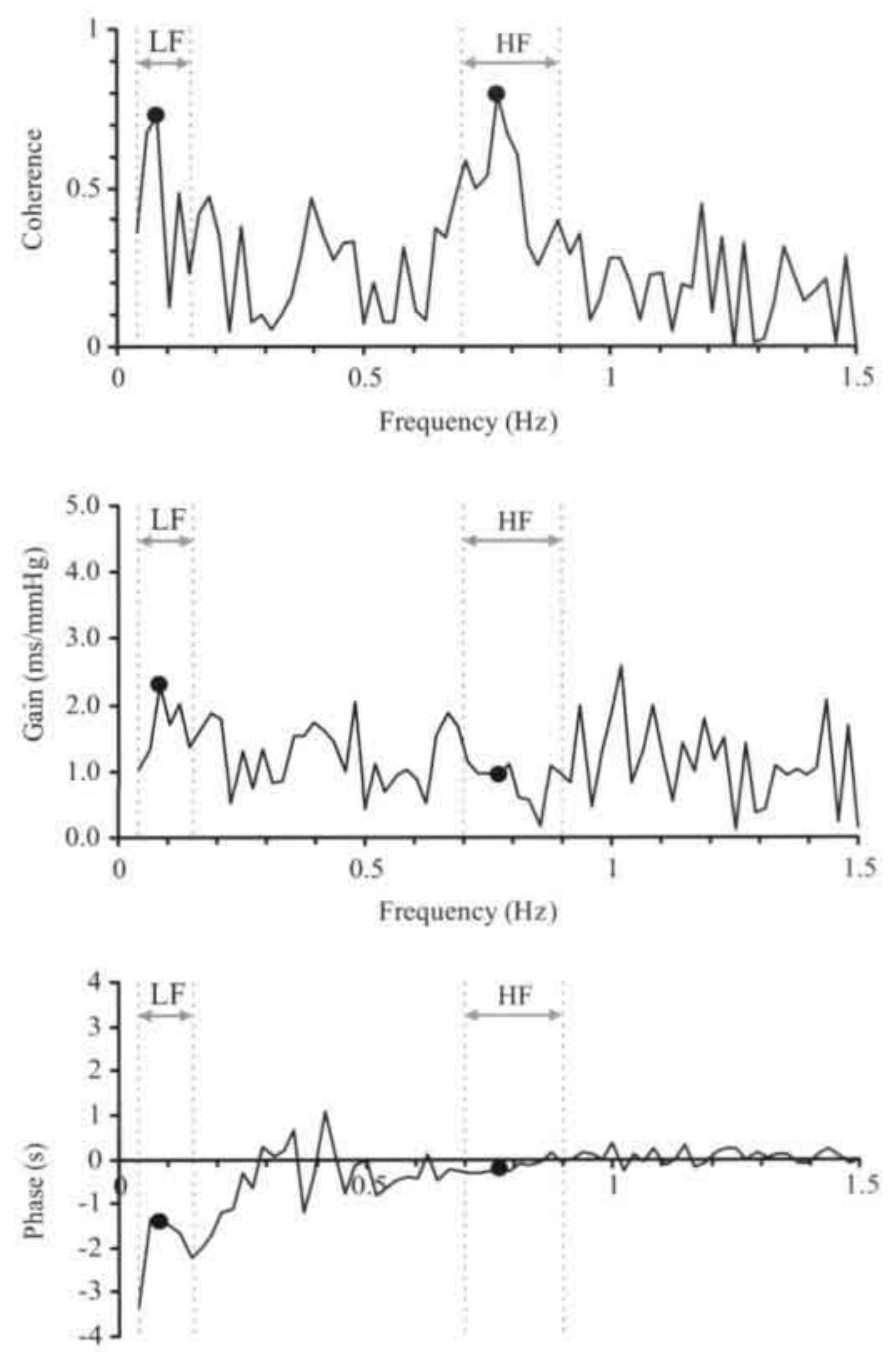

Frequency $(\mathrm{Hz})$

Figure 2. The coherence and transfer function parameters of the same infant as in Figure 1. This figure shows the coherence function for the linear relationship between R-R interval and SBP series (top) and corresponding transfer gain (middle) and transfer phase (bottom) as a function of frequency $(\mathrm{Hz}$ ). The vertical lines indicate the boundaries of the LF and HF bands. Note the high coherence values in both LF (0.73) and HF bands (0.79). The frequency value with highest coherence in LF was chosen to compute LF transfer gain (BRS) and phase (indicated as dots). LF transfer gain and phase were $2.3 \mathrm{~ms} / \mathrm{mmHg}$ and $1.5 \mathrm{~s}$, respectively. The negative phase relationship indicates that SBP fluctuations lead R-R interval changes by $1.5 \mathrm{~s}$ and probably related to $\mathrm{BR}$ activity. Likewise, at HF transfer gain and phase can be calculated, $0.9 \mathrm{~ms} / \mathrm{mmHg}$ and $0 \mathrm{~s}$, respectively. At HF phase is zero, indicating SBP and R-R interval fluctuations are oscillating together and not related to BR. 


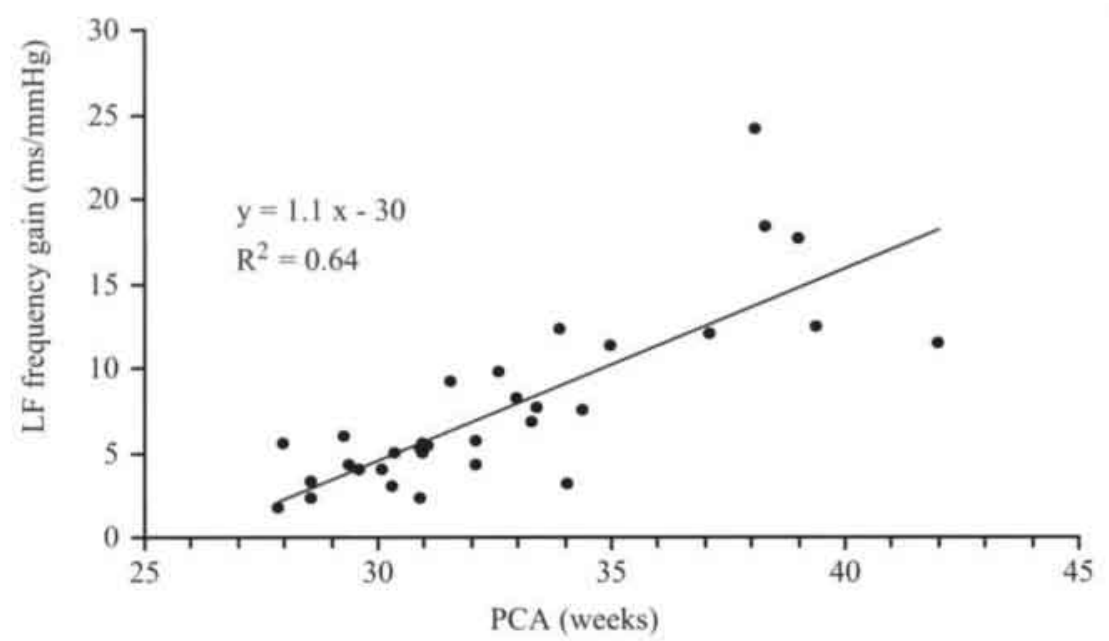

Figure 3. Correlation diagram between BRS and PCA. LF transfer gain, as an estimate for baroreceptor reflex sensitivity (BRS), correlated significantly with PCA.

Table 4: Transfer function analysis

\begin{tabular}{lcccc}
\hline & $\begin{array}{c}\text { PCA 28-32 } \\
(\mathbf{n = 1 6})\end{array}$ & $\begin{array}{c}\text { PCA 32-37 } \\
(\mathbf{n = 1 0})\end{array}$ & $\begin{array}{c}\text { PCA 37-42 } \\
(\mathbf{n = 6})\end{array}$ \\
\hline LF frequency & $0.07(0.06-0.10)$ & $0.08(0.06-0.10)$ & $0.08(0.08-0.11)$ \\
LF Coherence & $0.67(0.60-0.73)$ & $0.60(0.53-0.75)$ & $0.66(0.58-0.78)$ \\
LF gain (ms/mmHg) & $4.6 \quad(3.1-5.4)$ & $7.5 \quad(5.2-10.1)$ & $15.0 \quad(11.8-19.7)^{\#, \$}$ \\
LF phase $(\mathrm{s})$ & $-2.8 \quad(-3.6$ to -1.5$)$ & $-2.6 \quad(-5.5$ to -2.0$)$ & $-2.6 \quad(-3.9$ to -2.2$)$ \\
\hline
\end{tabular}

Low frequent (LF) transfer function analysis of the study group with a PCA between 28-32 wk; 32-37 wk; $37-42$ wk, respectively. The highest coherence value within the LF band is shown with the corresponding frequency, gain and phase. The LF gain was considered to be an estimate for baroreceptor reflex sensitivity. The negative phase values implicate blood pressure fluctuations lead R-R interval changes. Data are expressed as median and inter quartile range between brackets. Comparisons between groups were statistically evaluated with the Kruskal-Wallis $\mathrm{H}$ test and Mann Whitney $\mathrm{U}$ test:

@: $\mathrm{p}<0.01$ (PCA $32-37$ vs. 28-32);

\#: $\mathrm{p}<0.01$ (PCA $37-42$ vs. 33-37):

\$: $\mathrm{p}<0.01$ (PCA $37-42$ vs. 28-32). 
Table 5: Correlation coefficients between LF transfer gain and spectral power values

R-R interval series:

$\begin{array}{lll}\text { LF-RR } & +0.53 & \mathrm{p}<0.01 \\ \text { R-R interval } & +0.66 & \mathrm{p}<0.01 \\ \text { LF-RR } & +0.76 & \mathrm{p}<0.01 \\ \text { HF-RR } & +0.75 & \mathrm{p}<0.01 \\ \text { Total-RR } & +0.81 & \mathrm{p}<0.01 \\ \text { LF/HF ratio } & -0.31 & \mathrm{~ns} \\ & & \\ \text { SBP } & +0.39 & \mathrm{p}<0.05 \\ \text { LF-SBP } & -0.15 & \mathrm{~ns} \\ \text { HF-SBP } & -0.25 & \mathrm{~ns} \\ \text { Total-SBP } & -0.16 & \mathrm{~ns} \\ \text { HF-SBP } & -0.05 & \mathrm{~ns} \\ \text { Total-SBP } & -0.16 & \mathrm{~ns}\end{array}$

Correlation coefficient between LF transfer gain and spectral power values of R-R interval and SBP series. The total power was computed for the frequency band between 0.04 and $1.5 \mathrm{~Hz}$. The low frequency (LF) band was defined between 0.04 and $0.15 \mathrm{~Hz}$. The high frequency (HF) band was defined as the frequency range between the p-10 and p-90 centile of the individual respiratory frequency. Data are expressed as the Spearman's rho correlation coefficient with corresponding $\mathrm{p}$ value; not significant (ns).

\section{Discussion}

In this study we observed that R-R interval and SBP increased with PCA. These simple findings represent normal developmental physiology 17 . In addition, LF transfer gain (BRS) increased with PCA. In very preterm infants (PCA, 28-32 wk) the BRS was 4.6 $\mathrm{ms} / \mathrm{mmHg}$. The BRS value increased in more mature preterm infants (PCA, 32-37 wk) to $7.5 \mathrm{~ms} / \mathrm{mmHg}$ and increased further in full term babies (PCA, 37-42 wk) to 15 $\mathrm{ms} / \mathrm{mmHg}$. The increase in BRS significantly correlated with an increase in (LF-, HF-, and total) variability of R-R interval series, without a significant correlation in variability of SBP series.

Baroreceptor reflex sensitivity, animal data: Knowledge about the ontogeny of the BR relies almost exclusively on animal studies. The two primary species used to study vertebrate cardiovascular development, sheep and chickens, both possess a functional $\mathrm{BR}$ during fetal or embryonic life ${ }^{18-20}$. This suggests that either the BR is an important 
component of cardiovascular regulation during development or that it becomes functional in anticipation of birth. Studies in embryonic chickens suggest the latter, given that the BR response emerges very late in development (approximately $90 \%$ of incubation) and have lower gain compared with those in adults ${ }^{20}$. By contrast, fetal sheep possess a BR as early as $60 \%$ of gestation, with a decrease in BRS during development ${ }^{19,21}$. In fetal sheep, the arterial BP rises during the perinatal period which is accompanied by maturational changes in the neural and endocrine mechanisms that regulate $\mathrm{BP}^{21.22}$. The operating point of the ovine fetal $\mathrm{BR}$ increases towards term, while the sensitivity of the BR decreases as arterial BP rises with advancing gestational age in the sheep ${ }^{21}$. After birth, BRS continues to decrease as arterial rises to its resting postnatal values ${ }^{18.21}$. Animal data suggest that the development of the parasympathetic nervous system parallels the maturation of BRS with gestational age and postnatal life ${ }^{19}$. By contrast, an activated sympathetic system is dominantly present in the immediate postnatal period and subsequently decreases over time ${ }^{21}$. Taken together, although investigators disagree about the way in which BRS varies with pre- and postnatal maturation, they agree about the existence of a functioning BR during fetal life. Remarkably, this study shows both similarities with the sheep model (functioning BR at 26-27 wk, equivalent with $65 \%$ of gestation) and with the chicken model (progressive maturation of the BR with gestational age). However, unlike fetal sheep the BRS in human infants increases with PCA.

Baroreceptor reflex sensitivity, human infant data: Data about the ontogeny of the BR and functional maturation in the human infant is limited and conflicting ${ }^{17}$. This is partly caused by the limited experimental (pharmacological or mechanical) possibilities to challenge the BR in neonates. Passive head-up tilt test has been applied to neonates to measure responses in BP, HR and limb blood flow to body tilting. In preterm infants (gestational age, 26-37 wk), passive head-up tilt resulted in significant vasoconstriction of the lower limb with a slight fall in aortic BP and unchanged $\mathrm{HR}^{23}$. The inadequate ability to maintain BP and the lack of a tachycardia suggest that preterm infants lack the full integrated BR response as seen in adults. In term infants, however, a fall in BP was observed in conjunction with a tachycardia and a fall in limb blood flow, suggesting the presence of active reflex vasoconstriction ${ }^{24}$. Others, however, conclude that term as well as preterm babies (gestational age, 33-37 wk) show a well-developed HR response to passive head-up tilt ${ }^{25}$. A clear HR response to passive head-up tilt is present in fullterm babies after 2 hours of birth and progressively increases within the first postnatal day ${ }^{26}$. By contrast, some other studies do not show consistent evidence of a welldeveloped BR mediated HR response in the neonate ${ }^{27,28}$. The disparity in results may be, beside gestational age, explained by methodological differences, age at study, 
smoothness of tilting procedure, sleep state and the methods of measurements. Thus, the available studies of HR response to passive head-up tilt in human neonates suggest at least qualitatively a BR mediated HR response is present in early postnatal life.

Change in BRS is considered to be an important quantitative measure of BR maturation during development ${ }^{20,21}$. In contrast to the abundant data in animals only a few studies in the human infant have estimated the BRS quantitatively from spontaneously occurring fluctuations in BP and R-R interval series ${ }^{10-12}$. Notwithstanding different methodology (time domain or frequency domain analysis), the "spontaneous" BRS obtained from these studies show comparable values, ranging from approximately 3-5 $\mathrm{ms} / \mathrm{mmHg}$ (very preterm infant) to $10-15 \mathrm{~ms} / \mathrm{mmHg}$ (term infant) ${ }^{10-12}$. In addition, in the present study a strong and significant correlation was observed between PCA and BRS. Recently, a comparison of various techniques to estimate the BRS from spontaneously occurring fluctuations in BP and HR, showed strongly related results between the time sequence and LF transfer gain method ${ }^{29}$. Two studies, performed in mechanically ventilated and paralyzed human neonates undergoing major surgery, have estimated the pressor and depressor response of the BR by administering epinephrine and sodium nitroprusside, respectively ${ }^{30.31}$. In both studies, the pressor BR slope exceeded that of the depressor BR slope. In term neonates, the mean slopes were 11 (range 3-24) and $4 \mathrm{~ms} / \mathrm{mmHg}$ (range 2-12), respectively. These findings are consistent with results obtained from critically ill near-term neonates (gestational age, 35-42 wk) during and after extra-corporal membrane oxygenation in which BRS, derived from spontaneous fluctuations in $\mathrm{BP}$ and $\mathrm{HR}$, was higher during $\mathrm{BP}$ rise than during $\mathrm{BP}$ fall $^{32}$. In normal conscious humans, reflex parasympathetic stimulation and withdrawal primarily control $\mathrm{HR}$ responses to changes in $\mathrm{BP}^{33}$. Because cardiac slowing provoked by raising arterial BP is mediated primarily by an increase in parasympathetic discharge ${ }^{33}$, it is possible that parasympathetic stimulation results in a more marked (pressor) BR response than parasympathetic withdrawal (depressor) in the neonate. Conversely HR increase provoked by falling arterial BP appears to be mediated by increased $\beta$-adrenergic activity, as well as reduced vagal discharge ${ }^{33}$. However, sympathetic stimulation, while influencing basal HR, plays a minor role in BR mediated HR control. Previously we demonstrated a marked effect of atropine on BR mediated HR control, suggesting that an active parasympathetic nervous system is present in very preterm infants ${ }^{34}$.

Despite the methodological differences between several studies, we conclude that in the human infant: 1) a BR mediated HR response can be demonstrated in the immediate postnatal period, and 2) BRS is limited in very preterm infants but increases with advancing PCA. 
Mechanisms: BR mediated HR control involves three components: a) the afferent limb consisting of the baroreceptors, b) the integrative neurons of the medulla oblongata, and c) the efferent limbs originating from the vagal nuclei, the nucleus ambiguous and the dorsal moter nucleus of the vagal nerve. Studies in fetal sheep showed functioning baroreceptors and might suggest an intact afferent pathway of the reflex arc in mammalians, including the preterm infant ${ }^{18}$. Alternatively, a depressed BRS in very preterm infants might be caused by an impairment of central integration of baroreceptor input or the efferent limb limb of the reflex, namely the vagal innervation of the heart. Although vagal pathways from the vagal nuclei terminate on the sinus node, it is hypothesized that the fibers originating in the nucleus ambiguous are uniquely responsible for the HR variability, while the dorsal motor nucleus controls the tonic HR regulation ${ }^{35}$. It is possible that in preterm infants the vagal nervous system is not fully matured or has a different myelination or lacks the interconnections between the two vagal nuclei to ensure mature cardiorespiratory control $^{35-37}$.

To enhance the understanding of the underlying mechanisms of BRS we performed spectral analysis of R-R interval series and SBP series. With advancing PCA we observed higher (LF-, HF-, and total) spectral power values of R-R interval series. Like others, we observed a higher amount of HF relative to total spectral power with PCA (Table 2), suggesting that the parasympathetic contribution to modulate the HR increases with $\mathrm{PCA}^{38,39}$. By contrast, lower total and HF spectral power values of SBP series were observed in the term babies. Apparently at HF, the term infant is capable of adjusting SBP fluctuations by a more mature parasympathetic system. Furthermore, we observed that increase in BRS was correlated with an increase in (LF-, HF-, and total) variability of R-R interval series, without a significant correlation in variability of SBP series. However, BRS was also positively correlated with SBP and R-R interval (Table 5 ). Thus, we cannot exclude the possibility that the increase in BRS is merely the result of a different operating point of the BR curve due to increasing SBP with advancing PCA. Some investigators have shown that the LF/HF ratio, as an estimate of sympathovagal balance, decreases with $\mathrm{PCA}^{39.40}$. It is however questionable whether this ratio represents sympathovagal balance ${ }^{41}$. Furthermore, previous studies in (very) preterm infants show conflicting data regarding the applicability of this LF/HF ratio ${ }^{10,34}$. Also in this study we did not find a consistent relation between PCA or BRS and the LF/HF ratio of R-R interval series, respectively.

Thus, the observations of increase of R-R interval (decrease in HR), increase in (HF) spectral power of R-R interval series, and increase of BRS values with PCA indicate a progressive maturation of the parasympathetic nervous system with gestation. 
Methodological considerations: A limitation of the study group is its heterogeneity regarding the underlying pathology of the infants. Compared with term infants, preterm infants differ with respect to primary pulmonary diagnosis, surfactant administration, caffeine therapy for apnea of prematurity and indomethacine therapy for closing the ductus arteriosus before the measurements. Another limitation is the unequal data size between the different PCA groups. As a consequence of our restrictive policy to introduce invasive catheters in infants, especially recruiting cardiovascular stable nonasphyxiated term neonates with the presence of umbilical arterial catheters was difficult. A limitation is that PCA does not exclusively represent intra- or extra-uterine development because it reflects intrauterine (function of GA) as well as postnatal maturation. In addition, another limitation might be the effect of antenatal administration of steroids on glucocorticoid-dependent maturation of the BR and fetal HR variability. Antenatal glucocorticoids decrease BRS after birth in a preterm delivered sheep model ${ }^{42}$. In human fetuses antenatal glucocorticoids transitory lowers short and long-term fetal HR variability for 1-3 days after administration and normalizes at day $4{ }^{43}$. The period between antenatal glucocorticoid administration and postnatal measurement was at least 3 days in this study, and hence it is possible that our postnatal results might reflect the effect of antenatal steroids rather than normal development of the preterm infant. Finally, a limitation concerns the method of estimating BRS from spontaneously occurring fluctuations in R-R interval and SBP. With this method, information about BR mediated HR response is limited to the physiological gain and operating point, and the overall reflex parameters, such as the range of R-R interval response, level of the upper and lower plateaus, pressor and depressor gain, are not evaluated ${ }^{44}$.

\section{Conclusion}

Among regulatory mechanisms, the BR is the most prominent short-term compensator during arterial pressure challenges. A matured BR system is essential in avoiding hypotensive or hypertensive episodes. This study demonstrates that the capability of the BR to buffer spontaneous occurring physiological BP fluctuations by R-R interval changes is limited in very preterm infants, but increases with PCA. As a consequence, compensating larger BP changes due to intensive care management is presumable much more difficult to handle in the preterm infant. An immature BR function may thus contribute to conditions that predispose to pathological diseases in the preterm infant. $\mathrm{BR}$ maturation with gestation seems to be an effect of progressive parasympathetic 
activity. This study does not explain the postnatal maturation of BRS. However, with noninvasive techniques to measure beat-to-beat $\mathrm{BP}$, we aim to investigate this in the near future 45 . 


\section{References}

1. Stauss HM 2002 Baroreceptor reflex function. Am J Physiol 283:R284-R286

2. Smyth HS, Sleight P, Pickering GW 1969 Reflex regulation of arterial pressure during sleep in man: a quantitative method of assessing baroreflex sensitivity. Circ Res 24:109-121

3. Korner PI, West MJ, Shaw J, Uther JB 1974 "Steady state" properties of the baroreceptor heart rate reflex in essential hypertension in man. Clin Exp Pharmacol Physiol 1:65-76

4. Fritsch JM, Rea RF, Eckberg DL 1989 Carotid baroreflex resetting during drug-induced arterial pressure changes in humans. Am J Physiol 256:R549-R553

5. Akselrod S, Gordon D, Madwed JB, Snidman NC, Shannon DC, Cohen RJ 1985 Hemodynamic regulation: investigation by spectral analysis. Am J Physiol 249:H867-H875

6. de Boer RW, Karemaker JM, Strackee J 1987 Hemodynamic fluctuations and baroreflex sensitivity in humans: a beat-to-beat model. Am J Physiol 253:H680-H689

7. Robbe HW, Mulder LJM, Rüddel H, Langewitz WA, Veldman JBP, Mulder G 1987 Assessment of baroreceptor reflex sensitivity by means of spectral analysis. Hypertension 10:538-543

8. Honzíková N, Fišer B, Honzík J 1992 Noninvasive determination of baroreflex sensitivity in man by means of spectral analysis. Physiol Res 41:31-37

9. Head GA, Lukoshkova EV, Burke SL, Malpas SC, Lambert EA, Janssen BJA 2001 Comparing spectral and invasive estimates of baroreflex gain. IEEE Eng Med Biol Mag 20(2):43-52

10. Andriessen P, Koolen AMP, Berendsen RCM, Wijn PFF, ten Broeke EDM, Oei SG, Blanco CE 2003 Cardiovascular fluctuations and transfer function analysis in stable preterm infants. Pediatr Res 53:89-97

11. Drouin E, Gourmay V, Calamel J, Mouzard A, Rozé JC 1997 Assessment of spontaneous baroreflex sensitivity in neonates. Arch Dis Childhood 76:F108-F112

12. Gournay V, Drouin E, Rozé JC 2002 Development of baroreflex control of heart rate in preterm and full term infants. Arch Dis Child Fetal Neonatal Ed 86:F151-154

13. Kloosterman GJ 1970 On intrauterine growth. Int J Gynaec Obst 8:895-912

14. Prechtl HFR 1974 The behavioral states of the newborn infant. Brain Res 76:185-212

15. Jaffe RS, Fung DL 1994 Constructing a heart rate variability analysis system. J Clin Monit 10:45-58

16. Akselrod S 1995 Components of heart rate variability: basic studies. In: Malik M, Camm AJ (eds) Heart rate variability. Futura Publishing Company, New York, pp 147-163

17. Gootman PM 1991 Developmental aspects of reflex control of the circulation In: Gilmore JP, Zucker IH (eds) Reflex control of the circulation. CRC Press, Boca Raton, FL, pp 965-1027

18. Blanco CE. Dawes GS, Hanson MA, McCooke HB 1988 Carotid baroreceptors in fetal and newborn sheep. Pediatr Res 24:342-346

19. Segar JL 1997 Ontogeny of the arterial and cardiopulmonary baroreflex during fetal and postnatal life. Am J Physiol 273:R457-R471

20. Altimiras J, Crossley II DA 2000 Control of blood pressure mediated by baroreflex changes of heart rate in the chicken embryo (Gallus gallus). Am J Physiol 278:R980-R986

21. Segar JL, Hajduczok G, Smith BA, Merrill DC. Robillard JE 1992 Ontogeny of baroreflex control of renal sympathetic nerve activity and heart rate. Am J Physiol 263:H1819-H1826

22. Unno N, Wong CH, Jenkins SJ, Wentworth RA, Ding XY, Li C, Robertson SS, Smotherman WP, Nathanielsz PW 1999 Blood pressure and heart rate in the ovine fetus: ontogenic changes and effects of fetal adrenalectomy. Am J Physiol 276:H248-H256

23. Waldman S, Krauss AN, Auld PAM 1979 Baroreceptors in preterm infants: their relationship to maturity and disease. Develop Med Child Neurol 21:714-722

24. Picton-Warlow CG, Mayer FE 1970 Cardiovascular responses to postural changes in the neonate. Arch Dis Child 45:354-359 
25. Finley JP, Hamilton R, MacKenzie MG 1984 Heart rate response to tilting in newborns in quiet and active sleep. Biol Neonate 45:1-10

26. Chen CM, Tsai TC, Lan MC 1995 Effect of body tilting on physiological functions in healthy term neonates. Acta Paediatr 84:474-477

27. Holden K, Morgan JS, Krauss AN, Auld PAM 1985 Incomplete baroreceptor responses in newborn infants. Am J Perinatol 2:21-34

28. Moss AJ, Emmanouilides GC, Monset-Couchard M, Marcano B 1968 Vascular responses to postural changes in normal newborn infants. Pediatrics 42:250-254

29. Laude D, Elghozi J, Girard A, Bellard E, Bouhaddi M, Castiglioni P, Cerutti C, Cividjian A, Di Rienzo M, Fortrat JO, Janssen B, Karemaker JM, Leftheriotis G, Parati G, Persson PB, Porta A. Quintin L, Regnard J, Rudiger H, Stauss HM 2004 Comparison of various techniques used to estimate spontaneous baroreflex sensitivity (the EuroBaVar study). Am J Physiol 286:R226-R231

30. Murat I, Levron JC, Berg A, Saint-Maurice C 1988 Effects of fentanyl on baroreceptor reflex control of heart rate in newborn infants. Anesthesia 68:717-722

31. Murat I, Lapeyre G, Saint-Maurice C 1989 Isoflurane attenuates baroreflex control of heart rate in human neonates. Anesthesia 70:395-400

32. Buckner PS, Maidens JM, Finer NN 1993 Characterization of the neonatal heart rate baroreflex during and after ECMO. Early Hum Dev 32:49-61

33. Eckberg DL 1980 Nonlinearities of the human carotid baroreceptor-cardiac reflex. Circ Res 47:208216

34. Andriessen P. Janssen BJA, Berendsen RCM, Bambang Oetomo S, Wijn PFF, Blanco CE Cardiovascular autonomic regulation in preterm infants: the effect of atropine. In press, Pediatric Research

35. Porges SW 1995 Orienting in a defensive world: Mammalian modifications of our evolutionary heritage. A polyvagal theory. Psychophysiology 32:301-318

36. Taylor EW 1994 The evolution of efferent vagal control of the heart in vertebrates. Cardioscience 5:173-182

37. Sachis PN, Armstrong DL, Becker LE, Bryan AC 1982 Myelination of the human vagus nerve from 24 weeks postconceptional age to adolescence. J Neuropathol Exp Neurol 41:466:472

38. Clairambault J, Curzi-Dascalova L, Kauffmann F, Medigue C, Leffler C 1992 Heart rate variability in normal sleeping full-term and preterm neonates. Early Hum Dev 28:169-183

39. Chatow U, Davidson S, Reichman BL. Akselrod S 1995 Development and maturation of the autonomic nervous system in premature and full-term infants using spectral analysis of heart rate fluctuations. Pediatr Res 37:294-302

40. Mazursky JE, Birkett CL. Bedell KA, Ben-Haim SA, Segar JL 1998 Development of baroreflex influences on heart rate variability in preterm infants. Early Hum Dev 53:37-52

41. Eckberg DL 1997 Sympathovagal balance: a critical appraisal. Circulation 96:3224-3232

42. Segar JL, Lumbers ER, Nuyt AM, Smith OJ, Robillard JE 1998 Effect of antenatal glucocorticoids on sympathetic nerve activity at birth in preterm sheep. Am J Physiol 274:R160-170

43. Derks JB. Mulder EJH. Visser GHA 1995 The effects of maternal betamethasone administration on the fetus. Br J Obstet Gynaecol 102:40-46

44. Parlow J. Viale JP, Annat G, Hughson R, Quintin L 1995 Spontaneous cardiac baroreflex in humans: comparison with drug-induced responses. Hypertension 25:1058-1068

45. Andriessen P, Schoffelen RLM. Berendsen RCM, de Beer NAM, Oei SG, Wijn PFF, Blanco CE 2004 Noninvasive assessment of blood pressure variability in preterm infants. Pediatr Res 55:220-223 


\section{Chapter 9}

General discussion and perspectives 
Among regulatory mechanisms, the baroreceptor reflex is the most prominent shortterm compensatory mechanism during arterial pressure challenges ${ }^{1}$. This compensatory system results in an alteration of cardiac output and peripheral resistance via the cardiac and peripheral limb of the baroreceptor reflex, respectively. While progress has been made concerning regulation of the adult cardiovascular system, the level of information pertaining to the perinatal period is still quite limited. The mechanisms of cardiovascular control likely exist in the fetus and newborn, although considerable differences exist in the development of autonomic regulation of cardiovascular function among animal species because of varying rates of maturation of both peripheral and central components of the baroreceptor reflex arc. Because the majority of studies of fetal and neonatal cardiovascular regulation have been performed in animals, I was interested to explore the baroreceptor mediated heart rate reflex in the human neonate. In this thesis I present data of the baroreceptor reflex in neonates, varying from very preterm to term gestation and under varying clinical conditions. Rather than a summary of the consecutive studies I choose for an integrative discussion of the main findings from the different studies. The main findings address to the goals of the thesis and are presented in the following paragraphs.

\subsection{Methodology}

Since Akselrod performed frequency analysis on the fluctuation of R-R intervals in dogs, and pointed out the relation between the activity of the autonomic nervous system and the low frequency and high frequency peaks of the frequency domains, frequency analysis of R-R interval and blood pressure fluctuations has been performed widely ${ }^{2}$. Mostly these frequency analyses rely upon the Fourier transform. Fourier transform assumes stationary signals with identical spectral characteristics throughout the analysis window $^{3}$. In chapter 3 and 4 the Fourier technique is discussed in detail. In physiological conditions, however, signals stability (e.g. blood pressure, R-R interval) is difficult to ensure. Notably, very preterm infants show rapidly fluctuating heart rate and blood pressure due to motor activity, arousal state or immature autonomic regulation. Therefore, a compromise has to be chosen between the lowest frequency of interest (between 0.04 and $0.15 \mathrm{~Hz}$, associated with baroreceptor reflex activity), which determines the minimum window length, and the required accuracy for establishing the occurrence of high frequency of interest (between 0.4 and $1.5 \mathrm{~Hz}$, associated with respiratory activity), which determines the maximal length. In chapter 4 a customized filtering technique is presented which reduces the time window from 64 to 42 seconds. An advantage of the customized filtering technique is the reduced time window, which 
might be beneficial to investigate cardiovascular control in fetuses or very preterm infants showing rapidly changing arousal state. A disadvantage of the technique is that predefined spectral band definitions preclude individual spectral band analysis of respiratory associated fluctuations. The study of customized spectral band analysis compared with conventional Fourier analysis followed chronologically the four clinical studies. Therefore in all our clinical studies the conventional Fourier technique is used. Still Fourier transform or its derivates does not offer an easy assessment of its dynamics because of limitations inherent in its stationary assumption. An alternative mathematical method, wavelet transform, has been developed to overcome the limitation of stationary signals 4,5 . Wavelet transform allows a temporally localized analysis of the signal, giving access at any time to the status of heart rate variability when the balance of autonomous nervous equilibrium is suddenly modified by a drug or dynamic challenge. Wavelet transform thus allows analysis of non-stationary signals. Wavelet transform of heart rate variability has been applied to study the non-linear control system of heart rate in fetuses ${ }^{6}$. It would be interesting to develop a wavelet transform analysis in the future, because this would be a technique to explore baroreceptor mediated (non stationary) heart rate response to passive head-up tilt in neonates.

Cross-spectral analysis (or transfer function analysis) of the physiological signals offers an interesting technique to estimate the interrelation between spontaneously occurring beat-to-beat blood pressure and R-R interval fluctuations in the frequency domain and to quantify the baroreceptor mediated heart rate response ${ }^{7,8}$. With cross-spectral analysis the negative feedback (transfer function gain) and time-delay (transfer function phase) mechanisms of the cardiovascular system resulting in oscillations in heart rate and blood pressure can be calculated. With the spontaneous baroreceptor reflex method, information about baroreceptor reflex function is limited to the physiological gain and operating point, and the overall reflex parameters, such as the range of R-R interval response, (sub)threshold and saturation levels, pressor and depressor gain, are not evaluated ${ }^{1,9,10}$. In a recent paper it is shown that baroreceptor reflex sensitivity indices, derived from spontaneously occurring fluctuations in blood pressure and heart rate, did not show an adequate correlation with the pharmacological baroreceptor reflex gain or carotid distensibility ${ }^{11}$. Indeed, the difference between spontaneous baroreceptor reflex sensitivity slope and drug-induced slopes may reflect different aspects of the cardiac baroreceptor reflex. The gain of the drug-induced baroreceptor reflex is an index of the maximal recruitment of cardiac parasympathetic motorneurons as well as sympathetic premotorneurons during extreme conditions. By contrast, the gain of the spontaneous baroreceptor reflex sensitivity method indicates minimal recruitment by physiological 
pressure stimuli of only cardiac parasympathetic motorneurons under baseline conditions. Increase in spontaneous derived baroreceptor reflex sensitivity thus reflects a progressive parasympathetic activity of the cardiac vagal motorneurons. Other investigators have shown that - within the physiological range of blood pressure under resting conditions - the spontaneous method give similar results as the drug-induced method $^{10,12,13}$. In several studies (chapter 6-8) a wide range of spontaneous derived baroreceptor reflex sensitivity values was observed which correlated highly with gestational age: consistent low values in preterm infants and relatively high values in term babies. In addition, the values are in good agreement with results from others, although they used a different (time sequence) technique ${ }^{14,15}$ (chapter 2.4). Therefore I conclude that the spontaneous method is of value in assessing of baroreceptor reflex sensitivity in patients in whom other methods may be unsuitable.

I presented in chapter 5 the feasibility of a noninvasive beat-to-beat finger arterial blood pressure device (Finapres), applicated around the baby's wrist, to estimate blood pressure variability in preterm infants. This enables to investigate blood pressure changes in response to dynamic challenges, i.e. passive head-up tilt to unload the baroreceptor. Finapres thus provides a noninvasive tool for investigating the autonomic cardiovascular regulation in healthy neonates. However, a limitation of the wrist application of the finger cuff device is that the used (adult) physiocal algorithm for servo calibration is not developed for cuff application at the wrist. Therefore, the algorithm does not always succeed in finding (at relatively low blood pressure values) the correct cuff pressure for vascular unloading. The main reason to use this wrist application is that there are no appropriate finger cuffs for babies. Recently I participated in a research program of the TNO Institute of Applied Physics (Biomedical Instrumentation, Amsterdam, The Netherlands) to develop an appropriate baby finger cuff. The first results of several prototypes miniature finger cuffs are presented in June 2004 at the fourteenth European Meeting on Hypertension in Paris, France ${ }^{16}$. The abstract of this study is presented in appendix B.

\subsection{The baroreceptor reflex mediated cardiovascular fluctuations in preterm infants}

As presented in chapters 6-8, spontaneously occurring fluctuations in arterial blood pressure and R-R interval are observed at relatively low (approximately $0.1 \mathrm{~Hz}$ ) and relatively high frequency (related to the respiratory rate, range $0.4-1.5 \mathrm{~Hz}$ ), even in very preterm infants. 
Concerning the origin of low frequent fluctuations, several models have been proposed to explain the low frequent oscillation of R-R interval and blood pressure. Several investigators proposed the idea that a brainstem neural oscillator independently generates the low frequent activity ${ }^{17}$. This is supported by the presence of low frequent oscillation in R-R interval in patients with heart failure ${ }^{18}$. However, most investigators explain the low frequent oscillation of R-R interval and blood pressure in terms of a baroreceptor reflex mechanism $7.8,19,20$. Strong support for the baroreceptor reflex model comes from evidence that blocking or removal of different sections of the feedback loop reduce the strength of the low frequency oscillation ${ }^{21}$. It is therefore postulated that baroreceptor control may be the main cause of low frequent R-R interval fluctuations in response to blood pressure fluctuations generated by an oscillation of the sympathetic vasomotor tone ${ }^{22}$. Spontaneous fluctuations in R-R interval and blood pressure are used to investigate reflex cardiovascular control in closed-loop conditions. In human adults the delay of (low frequent) R-R interval relative to blood pressure fluctuations is approximately 2 seconds $s^{8,20,23}$. The observations in preterm infants are in agreement with this view. I observed clear fluctuations in systolic blood pressure and R-R interval at a frequency of approximately $0.1 \mathrm{~Hz}$ with a consistent temporal phase delay of approximately 4 seconds of R-R interval change relative to blood pressure change, indicating a baroreceptor reflex mechanism. The greater time delay between low frequent fluctuations of blood pressure and R-R interval might be caused by a slower conductance of autonomic nerves in the very preterm infants 24,25 .

The origin of high frequent blood pressure fluctuations is probably caused by rhythmic changes of intrathoracic pressure due to respiratory mechanisms ${ }^{21,26}$. Mechanical cardiopulmonary coupling has been suggested as a source of respiration-related heart rate variability through the Bainbridge reflex ${ }^{27}$. This reflex is initiated by atrial mechanoreceptors and uses, like the baroreceptor reflex, efferent sympathetic and parasympathetic pathways to modulate heart rate in response to changes in central venous pressure. Respiratory changes in central blood volume cause corresponding respiratory fluctuations in cardiac autonomic nervous system activity via the Bainbridge reflex. In preterm infants (chapters 6-8) the temporal phase relation between blood pressure and R-R interval at the respiratory associated high frequency range is close to zero seconds, suggesting that blood pressure and R-R interval fluctuations occur simultaneously and are not related to a reflex mechanism. 


\subsection{Autonomic nervous control of the baroreceptor reflex}

The R-R interval fluctuations are the result of sympathetic and vagal efferent activity on the sino-atrial node. Because of the limited ability of the heart to respond to sympathetic influences at the respiratory rate (chapter 2), it is thought that the respiratory-associated oscillation in heart rate is mediated via the vagal nerve only. This is supported by the observation in human adults that vagal blockade with atropine abolishes the respiratory associated oscillation in heart rate ${ }^{23}$. The origin of low frequent R-R interval fluctuations is uncertain, because different studies based on similar approaches have led to contradictory interpretations, varying from greater role either to sympathetic ${ }^{28}$ or vagal control ${ }^{29-31}$. The atropine study (chapter 7 ) showed that a significant vagal tone is already present in the first hours after birth: atropine increased heart rate and decreased variability of R-R interval series. Remarkably, atropine predominantly decreased the low frequent fluctuations of R-R interval series without affecting the low frequent fluctuations in blood pressure. Thus, the atropine study showed that baroreceptor reflex mediated parasympathetic control of heart rate is of significance for the cardiovascular control in the early postnatal period of respiratory distressed preterm infants. The decline in heart rate and increase in heart rate variability with gestational age (chapter 8) suggest a further progressive maturation of the parasympathetic nervous system.

My interpretation that a high $\mathrm{LF} / \mathrm{HF}$ ratio indicates an unbalance between the two branches of the autonomic nervous system towards sympathetic activity needs to be reconsidered ${ }^{32}$ (chapter 6 ). The assumed rationale for this mathematical ratio are 1 ) low frequent fluctuations of R-R interval are importantly mediated by fluctuations of sympathetic nerve activity, 2) respiratory associated high frequent fluctuations are almost exclusively mediated by fluctuations in vagal cardiac nerve activity, and 3) physiological interventions tend to provoke reciprocal changes of sympathetic and parasympathetic efferent nerve activity ${ }^{33}$. Sympathovagal balance, the ratio of these periodicities, is taken to reflect the balance between the opposing neural mechanisms ${ }^{33}$. As a consequence, a change of either the numerator or the denominator will change the ratio. Thus, a reduction of low frequent with no change of high frequent spectral power will reduce the ratio, and conversely a reduction of high frequent with no change of low frequent spectral power will increase the ratio. Studies in human adults, however, has shown that vagal contribution to low frequent fluctuations in R-R interval is very important 29.30 . Indeed following atropine in preterm infants, the low frequent spectral power of R-R interval was much more decreased than the high frequent spectral power and the LF/HF ratio decreased accordingly (chapter 7). Therefore, it is not very likely 
that this ratio reflects sympathovagal balance in preterm infants. On the contrary, following atropine the $\mathrm{LF} / \mathrm{HF}$ ratio might rather reflect a pure sympathetic state.

\subsection{Development of the baroreceptor reflex mediated heart rate response}

Because change in baroreceptor reflex sensitivity is considered to be an important measure of baroreceptor reflex maturation during fetal development ${ }^{34}$, low frequent transfer gain, as an estimate of baroreceptor reflex sensitivity, was investigated in several studies. Preterm infants (gestational age, 28-32 wk) showed a baroreceptor reflex sensitivity of approximately $4-5 \mathrm{~ms} / \mathrm{mmHg}$ within the first postnatal week (chapter 6, 7, and 8). The gain increased to $7.5 \mathrm{~ms} / \mathrm{mmHg}$ in more mature preterm infants (gestational age, 32-37 wk), and increased further to $15 \mathrm{~ms} / \mathrm{mmHg}$ in full term infants (chapter 8).

A matured baroreceptor reflex system is essential in avoiding hypotensive or hypertensive episodes. Apparently, the capability of the baroreceptor reflex to buffer spontaneous occurring physiological blood pressure fluctuations by $\mathrm{R}-\mathrm{R}$ interval changes is limited in very preterm infants, but increases with age. As a consequence, compensating larger blood pressure changes due to intensive care management is presumable much more difficult to handle in the preterm infant. An immature baroreceptor reflex function may thus contribute to conditions that predispose to pathological diseases in the preterm infant such as cerebral hemorrhage. Baroreceptor reflex maturation with gestation seems to be an effect of progressive parasympathetic activity.

There are similarities (functional baroreceptor reflex even at a very preterm stage; progressive parasympathetic maturation) and discrepancies (increasing baroreceptor reflex sensitivity with gestational age in human infants, decreasing baroreceptor reflex sensitivity with age in sheep) between the results obtained from human infants (chapter 6-8) and the standard mammalian example of cardiovascular research, the sheep ${ }^{34-36}$. The apparent contradiction of baroreceptor reflex sensitivity between the human infant and sheep may be the result of different causes. First, unlike animal studies it is not possible to investigate exclusively the fetal development of baroreceptor reflex in humans. The studies in human infants represent intra-uterine and extra-uterine maturation. Thus, results may be biased by the transition from fetal to newborn life which is known to be associated with hemodynamic adjustments, including changes in heart rate, blood pressure and peripheral vascular resistance, and modulated by catecholamine release and autonomic nervous control ${ }^{37-39}$. Second, as explained earlier 
(chapter 9.2), it is not possible with the use of transfer function technique to construct a full heart rate - blood pressure relationship with threshold, operating point and saturation levels. Most animal studies use vaso-active drugs to change blood pressure, measure heart rate response and construct a baroreceptor reflex curve between blood pressure and heart rate. Low frequent transfer gain reflects only minimal gain at a physiologic (operating) blood pressure level and does not represent maximal vagal activity. Third, antenatal administration of glucocorticoids in fetal sheep decreases baroreflex function ${ }^{40}$. It is therefore possible that the baroreceptor reflex sensitivity in preterm infants reflects the effect of antenatal administration of glucocorticoids rather than normal development.

As discussed in chapter 2.5 and chapter 8 , data about the development of the baroreceptor reflex in the human neonate are limited. This is partly because of the limited pharmacological possibilities to challenge the baroreceptor reflex. Changing body position is a noninvasive and powerful way to unload the baroreceptor and to stimulate sympathetic activity ${ }^{20}$. Although contradictory reports concerning the effects of changing body position of the newborn have been obtained, it is likely that a baroreceptor reflex mediated heart rate response is present in early postnatal life of term infants (chapter 2.5). The limitation of these studies, however, is that nearly all studies in human neonates investigated only heart rate response to body position. With the use of noninvasive techniques to measure beat-to-beat changes in blood pressure (Finometer, successor of Finapres, chapter 5) future research of investigating heart rate and blood pressure variability before and after passive-head up tilting is possible in healthy infants. In this way the effect of postnatal maturation may be studied. Recently the Department of Technology of Máxima Medical Center developed a specially designed tilt table for neonates, illustrated in appendix $\mathrm{C}$.

\subsection{Perspectives: fetal programming and future research}

Two systematic reviews, based on nearly 100 studies in more than 400.000 subjects of all ages (0-84 years) and races in many different countries, support an inverse relationship between birth weight and systolic blood pressure in children and in adults ${ }^{41,42}$. Many animal studies have shown that growth retardation induced by intrauterine stress may lead to hypertension and other cardiovascular disorders ${ }^{43-46}$. Studies in the early perinatal period are of interest from the view of physiological mechanism underlying the association and possible clues for prevention. Several mechanisms have been proposed in linking reduced fetal growth and increased blood 
pressure: (1) decreased placental 11B-hydroxysteroid dehydrogenase activity to protect the fetus from maternal glucocorticoid hormone ${ }^{47,48}$, (2) persisting changes in vascular structure, including loss of elasticity in vessel walls ${ }^{49}$, (3) impairment of renal development ${ }^{50}$, (4) endothelial dysfunction with impairment in nitric oxide mediated vasodilatation ${ }^{51,52}$, and (5) cardiovascular autonomic dysfunction ${ }^{53}$. From these mechanisms we focus on cardiovascular autonomic regulation and how this might play a role in elevating blood pressure.

The association between low birth weight and cardiovascular autonomic dysfunction is supported by several observational human studies. First, an association is found between low birth weight and a high resting pulse rate in adult life ${ }^{53}$. A high resting heart rate under standardized conditions may suggest a disturbance of the balance between the sympathetic and parasympathetic branches of the autonomic nervous system $^{54}$. Second, in adolescent subjects low birth weight was strongly associated with shorter pre-ejection period and a larger pre-ejection period reactivity in response to stress tasks, which are indicative of increased sympathetic nervous system activity ${ }^{55}$. In addition, this study showed that a shorter pre-ejection period was related to higher blood pressure and statistically explained a large part of the association between birth weight and blood pressure. Third, children (age, 4-18 years) who had lower birth weight not only have higher 24-hours ambulatory blood pressure values but also higher blood pressure variability ${ }^{56}$. Increased variability in blood pressure indicates abnormal cardiovascular control and has been linked to the development of hypertension-related organ damage ${ }^{57}$. Finally, growth retarded fetuses and infants show higher heart rate and lower heart rate variability which might indicate abnormal cardiovascular control ${ }^{58-61}$. The association between low birth weight, increased sympathetic activity and hypertension is consistent with several experimental animal studies. First, chronic moderate hypoxia (from day 6 of incubation) in the chick embryo causes fetal growth retardation and stimulates peripheral cardiovascular sympathetic nerve development at day 19 of incubation ${ }^{62}$. Second, in spontaneously hypertensive rats the pathophysiology of essential hypertension seems to be characterized by permanent damping of the spontaneous blood pressure fluctuations modified only when an acute perturbation (acute blood loss) is applied and the renin-angiotensin system is activated ${ }^{63,64}$. Under these provocative conditions an exaggerated response, demonstrated by increasing slow blood pressure fluctuations, is observed even in the pre-hypertensive phase of spontaneously hypertensive rats. Blockade of $\alpha$-sympathetic by prazosin attenuated the increase of low frequent fluctuations in blood pressure. These data indicate that the basic autonomic dysfunction is already present in prehypertensive young rats and which most probably originates from $\alpha$-sympathetic abnormality. Third, in female rats 
intrauterine growth retardation is associated with increased sympathetic nervous activity and impaired glucose tolerance in adult life ${ }^{65}$. Fourth, mild maternal undernutrition alters fetal cardiovascular development, producing a resetting of the baroreceptor reflex control mechanism, even in the absence of fetal growth retardation ${ }^{66}$. Finally, experimental evidence in rats suggests that activation of the renin-angiotensin system is an important element of hypertension programmed during fetal life ${ }^{67.68}$. Recently it has been shown that in the offspring of protein restricted pregnant dams, in utero programmed hypertension is associated with an activated reninangiotensin system together with increased low and high spectral power in blood pressure variability and impaired arterial baroreflex mediated heart rate control ${ }^{69}$. Following angiotensin-converting enzyme inhibitor, the blood pressure-heart rate baroreceptor reflex curve is reset to lower pressure in protein restricted rats ${ }^{69}$.

In summary, several experimental studies indicate the importance of impaired baroreceptor reflex function in fetal programming of hypertension. However, we do not know the effects of growth retardation on baroreceptor function in the human infant. With spectral analysis of heart rate and blood pressure relevant questions could be solved that help us to give more insight in important aspects of fetal programming. Therefore future studies of baroreceptor reflex in the human infant may focus on low birth weight or growth retarded infants and include the following hypotheses or questions: 1) increased blood pressure variability in growth-retarded infants might indicate an impaired blood pressure regulation, and 2) a decreased baroreceptor reflex sensitivity in growth-retarded infants might indicate less vagal modulation and probably indicates a sympathovagal balance towards the sympathetic nervous system. These hypotheses might be solved with the following techniques and procedures:

- spectral power analysis of blood pressure and R-R interval series;

- transfer function analysis: coherence, transfer gain, and phase;

- atropine study in growth-retarded infants: following atropine, a pure sympathetic state is achieved, and spectral power analysis of blood pressure and R-R interval series may be compared with controls;

- passive head-up tilt to unload the baroreceptor and to stimulate the sympathetic nervous system; measuring beat-to-beat blood pressure (noninvasive, Finometer) and $\mathrm{R}-\mathrm{R}$ interval to calculate the baroreceptor reflex mediated R-R response; this noninvasive technique gives the opportunity to investigate 'healthy' low birth weight or growth retarded term neonates and to compare these results with controls. 


\section{References}

1. Sagawa K. Baroreflex control of systemic arterial pressure and vascular bed. In: Shepherd JT, Abboud FM (eds.) Handbook of physiology. Section 2, volume III, part 2. Bethesda, MD, American Physiological Society, 1983, p 453-496

2. Akselrod S, Gordon D, Ubel FA, Shannon DC, Berger AC, Cohen RJ. Power spectrum analysis of heart rate fluctuation: a quantitative probe of beat-to-beat cardiovascular control. Science 1981;213(4504):220-222

3. Jaffe RS, Fung DL. Constructing a heart rate variability analysis system. J Clin Monit 1994;10:4558

4. Pichot V, Gaspoz JM, Molliex S, Antoniadis A, Busso T, Roche F, Costes F, Quintin L, Lacour JR, Barthélémy JC. Wavelet transform to quantify heart rate variability and to assess its instantaneous changes. J Appl Physiol 1999;86:1081-1091

5. Lotric MB, Stefanovska A, Stajer D, Urbancic-Rovan V. Spectral components of heart rate variability determined by wavelet analyis. Physiol Meas 2000, 21(4): 441-457

6. Kimura Y, Okamura K, Wantanbe T, Yaegashi N, Uehara S, and Yajima A. Time-frequency analysis of fetal heartbeat fluctuation using wavelet transform. Am J Physiol 275: H1993-H1999, 1998

7. de Boer RW, Karemaker JM, Strackee J. Relationships between short-term blood pressure fluctuations and heart rate variability in resting subjects. I: a spectral analysis approach Med Biol Eng Comput $1985 ; 23: 352-358$

8. de Boer RW, Karemaker JM, Strackee J. Hemodynamic fluctuations and baroreflex sensitivity in humans: a beat-to-beat model. Am J Physiol 1987;253:H680-H689

9. Korner PI, Wets MJ, Shaw J, Uther JB. "Steady-state" properties of the baroreceptor-heart rate reflex in essential hypertension in man. Clin Exp Pharmacol Physiol 1974:1:65-76

10. Parlow J, Viale JP, Annat G, Hughson R, Quintin L. Spontaneous cardiac baroreflex in humans: comparison with drug-induced responses. Hypertension 1995;25:1058-1068

11. Lipman RD Salisbury JK. Taylor JA. Spontaneous indices are inconsistent with arterial baroreflex gain. Hypertension 2003: 42:481-487

12. Robbe HWJ, Mulder LJM, Rüddel H, Langewitz WA, Veldman JBP, Mulder G. Assessment of baroreceptor reflex sensitivity by means of spectral analysis. Hypertension 1987:10:538-543

13. James MA, Panerai RB, Potter JF. Applicability of new techniques in the assessment of arterial baroreflex sensitivity in the eldery: a comparison with established pharmacological methods. Clin Sci 1998;94(3):245-253

14. Drouin E, Gourmay V, Calamel J, Mouzard A, Rozé JC. Assessment of spontaneous baroreflex sensitivity in neonates. Arch Dis Childhood 1997;76:F108-F112

15. Gournay V, Drouin E, Rozé JC. Development of baroreflex control of heart rate in preterm and full term infants. Arch Dis Child Fetal Neonatal Ed 2002;86:F151-154

16. Schraa O, Andriessen P, Smit B, van den Bosch-Ruis W, van Koppen M, Settels JJ. Feasibility of continuous noninvasive blood pressure measurements in infants. J Hypertens 2004;22 (suppl 2):S280

17. Montano N, Gnecchi-Ruscone T, Porta A, Lombardi F, Malliani A, Barman SM. Presence of vasomotor and respiratory rhythms in the discharge of single medullary neurons involved in the regulation of cardiovascular system. J Auton Nerv Syst $1996 ; 57: 116-122$

18. Cooley RL, Montano N, Cogliati C, van de Borne P, Richenbacher W, Oren R, Somers VK. Evidence for a central origin of the low-frequency oscillation in RR-interval variability, Circulation 1998; 98:556-561

19. Bernardi L, Leuzzi S, Radaelli A, Passino C, Johnston JA, Sleight P. Low-frequency spontaneous fluctuations of R-R interval and blood pressure in consciuos humans: a baroreceptor or central phenomenon? Clin Sci 1994:87:649-654 
20. Cooke WH, Hoag JB, Crossman AA, Kuusela TA, Tahvanainen KUO, Eckberg DL. Human responses to upright tilt: a window on central autonomic integration. J Physiol 1999:517.2: 617-628

21. Malpas SC. Neural influences on cardiovascular variability: possibilities and pitfalls. Am J Physiol 2002:282:H6-H20

22. Cevese A. Gulli G, Polati E, Gottin L, Grasso R. Baroreflex and oscillation of heart period at $0.1 \mathrm{~Hz}$ studied by alpha-blockade and cross-spectral analysis in healthy humans. J Physiol 2001:531:235244

23. Saul JPh, Berger RD, Albrecht P, Stein SP, Hui Chen M, Cohen RJ. Transfer function analysis of the circulation: unique insights into cardiovascular regulation. Am J Physiol 1991;261:H1231-H1245

24. Sachis PN, Armstrong DL, Becker LE, Bryan AC. Myelination of the human vagus nerve from 24 weeks postconceptional age to adolescence. J Neuropathol Exp Neurol 1982:41:466:472

25. Taylor EW. The evolution of efferent vagal control of the heart in vertebrates. Cardioscience 1994:5:173-182

26. Metsälä T, Siimes A, Antila K, Välimäki I. Association of breathing movements to the variability of heart rate and blood pressure in foetal lambs. Acta Physiol Scand 1993;147:213-219

27. Barbieri R, Triedman JK. Saul JP. Heart rate control and mechanical cardiopulmonary coupling to assess central volume: a systems analysis Am J Physiol 2002;283:R1210-1220

28. Pagani M, Montano N, Porta A, Malliani A, Abboud F, Birkett C, Somers VK. Relationship between spectral components of cardiovascular variabilities and direct measures of muscle sympathetic nerve activity in humans. Circulation 1997:95:1441-1448

29. Pomeranz B, Macaulay RJB, Caudill MA, Kutz I, Adam D, Gordon D, Kilborn KM, Barger AC, Shannon DC, Cohen RJ. Benson H. Assessment of autonomic function in humans by heart rate spectral analysis. Am J Physiol 1985:248:H151-H153

30. Brown TE, Beightol LA, Koh J, Eckberg DL. Important influence of respiration on human R-R interval power spectra is largely ignored. J Appl Physiol 1993;75(5):2310-2317

31. Taylor JA, Carr DL, Myers CW, Eckberg DL. Mechanisms underlying very-low-frequency RRinterval oscillations in humans. Circulation 1998;98:547-555

32. Eckberg DL. Sympathovagal balance: a critical appraisel. Circulation 1997:96:3224-3232

33. Task Force of the European Society of Cardiology and the North American Society of pacing and electrophysiology. Heart rate variability: standards of measurement, physiological interpretation, and clinical use. Circulation 1996;93:1043-1065

34. Segar JL. Ontogeny of the arterial and cardiopulmonary baroreflex during fetal and postnatal life. Am J Physiol 1997;273:R457-R471

35. Assali NS, Brinkman CR 3rd, Woods JR, Dandavino A. Nuwayhid B. Development of neurohumoral control of fetal, neonatal, and adult cardiovascular functions. Am J Obstet Gynecol 1977;129:748759

36. Woods JR. Dandavino A. Murayama K, Brinkman CR. Assali NS. Autonomic control of cardiovascular functions during neonatal development and in adult sheep. Circ Res 1977;40:401-407

37. Dawes GS. Changes in the circulation at birth. Br Med Bull 1961;17:148-153

38. Langercrantz H, Bisoletti P. Catecholamine release in the newborn at birth. Pediatr Res 1973;11:889893

39. Segar JL, Mazursky JE, Robillard JE. Changes in ovine renal sympathetic nerve activity and baroreflex function at birth. Am J Physiol 1994 ;267 :H1824-H1832

40. Segar JL, Lumbers ER, Nuyt AM, Smith OJ, Robillard JE. Effect of antenatal glucocorticoids on sympathetic nerve activity at birth in preterm sheep. Am J Physiol 1998;274:R160-170

41. Law CM. Shiell AW. Is blood pressure inversely related to birth weight? The strength of evidence from a systematic review of the literature. J Hypertens 1996; 14:935-941 
42. Huxley RR, Shiell AW, Law CM. The role of size at birth and postnatal catch-up growth in determining systolic blood pressure: a systematic review of the literature. J Hypertens 2000;18:815831

43. Persson E, Jansson T. Low birth weight is associated with elevated adult blood pressure in the chronically catheterized guinea-pig. Acta Physiol Scand 1992;145:195-196

44. Langley SC, Jackson AA. Increased systolic blood pressure in adult rats induced by fetal exposure to maternal low protein diets. Clinical Science 1994;86:217-222

45. Ozaki T, Hawkins P, Nishina H, Steyn C, Poston L, Hanson MA. Effects of undernutrition in early pregnancy on systemic small artery function in late-gestation fetal sheep. Am J Obstet Gynecol 2000 Nov; $183(5): 1301-7$

46. Brawley L, Itoh S, Torrens C, Barker A, Bertram C, Poston L. Hanson M. Dietary protein restriction in pregnancy induces hypertension and vascular defects in rat male offspring. Pediatr Res 2003;54:83-90

47. Edwards CRW, Benediktsson R, Lindsay RS, Seckl JR. Dysfunction of placental glucocorticoid barrier: link between fetal environment and adult hypertension? Lancet 1993:341:355-357

48. Benediktsson R, Lindsay RS, Noble J, Seckl JR, Edwards CRC. Glucocorticoid exposure in utero: new model for adult hypertension Lancet 1993;341:339-341

49. Martyn CN et al. Growth in utero, adult pressure, and arterial compliance. Br Heart J 1995:73:116121

50. Brenner BM, Chertow GM. Congenital oligonephropathy and the etiology of adult hypertension and progressive renal injury. Am J Kid Dis 1994;23:171-5

51. Martin H, Gazelius B, Norman M. Impaired Acetylcholine-induced vascular relaxation in low birth weight infants: implications for adult hypertension? Pediatr Res 2000:47:457-462

52. Ruijtenbeek K, Kessels LC, de Mey JG, Blanco CE. Chronic moderate hypoxia and protein malnutrition both induce growth retardation, but have distinct effects on arterial endotheliumdependent reactivity in the chicken embryo. Pediatr Res 2003:53(4):573-9

53. Phillips DIW, Barker DJP. Association between low birth weight and high resting pulse in adult life: is the sympathetic nervous system involved in programming the insulin resistance syndrome? Diabet Med 1997: 14:673-7

54. Goldberger J. Sympathovagal balance: how should we measure it? Am J Physiol 1999;45:H1273$\mathrm{H} 1280$

55. Uzzerman RG, Stehouwer CDA, de Geus EJ, van Weissenbruch MM, Delemarre-van de Waal HA. Boomsma DI. Low birth weight is associated with increased sympathetic activity. Circulation 2003; 108:566-571

56. Lurbe E, Torro I, Rodríguez C, Alvarez V, Redón J. Birth weight influences blood pressure values and variability in children and adolescents. Hypertension 2001;38:389-393

57. Mancia G, Giannasttasio C, Failla M. Sega R. Parati G. Systolic blood pressure and pulse pressure: role of 24-h mean value and variability in the determination of organ damage. J Hypertens 1999;17(suppl 5):S55-S6I

58. Breborowicz G, Moczko J, Gadzinowski J. Quantification of the fetal heart rate variability by spectral analysis in growth-retarded fetuses. Gynecol Obstet Invest 1988:25:186-191

59. Robinson SM, Wheeler T, Hayes MC, Barker DJP, Osmond C. Fetal heart rate and intrauterine growth. Br J Obstet Gynaecol 1991;98:1223-1227

60. Spassov L, Curzi-Dascalova L, Clairambault J, Kauffmann F, Eiselt M, Medigue C, Peirano P. Heart rate and heart rate variability during sleep in small-for-gestational age newborns. Pediatr Research 1994:35:500-505

61. Jackson JA, Wailoo MP, Thompson JR, Petersen SA. Early physiological development of infants with intrauterine growth retardation Arch Dis Child Fetal Neonatal Ed 2004:89:F46-F50 
62. Ruijtenbeek K, le Noble FAC, Janssen GMJ, Kessels CGA, Fazzi GE, Blanco CE, de Mey JGR. Chronic hypoxia stimulates periarterial sympathetic nerve development in the chick embryo. Circulation 2000;102:2892-2897

63. Akselrod $\mathrm{S}$, Eliash $\mathrm{S}, \mathrm{Oz} \mathrm{O}$, Cohen $\mathrm{S}$. Hemodynamic regulation in SHR: investigation by spectral analysis. Am J Physiol 1987;253:H176

64. Akselrod $\mathrm{S}, \mathrm{Oz} \mathrm{O}$, Eliash $\mathrm{S}$. Neural and humoral factors in regulation of blood pressure in the investigation of essential hypertension. In: Di Rienzo M (ed.) Blood pressure and heart rate variability, IOS Press, Amsterdam, 1993, pp192-206

65. Jansson T, Lambert GW. Effect of intrauterine growth restriction on blood pressure, glucose intolerance and sympathetic nervous system activity in the rat at 3-4 months of age. J Hypertens 1999:17:1239-1248

66. Hawkins P, Steyn C, Ozaki T, Saito T, Noakes DE. Hanson MA. Effects of maternal undernutrition in early gestation on ovine fetal blood pressure and cardiovascular reflexes. Am J Physiol 2000;279:R340-R348

67. Langley-Evans SC, Jackson AA. Captopril normalizes systolic blood pressure in rats with hypertension induced by fetal exposure to maternal low protein diets. Comp Biochem Physiol A Physiol 1995; 110:223-228

68. Sherman RC, Langley-Evans SC. Antihypertensive treatment in early postnatal life modulates prenatal dietary influences upon blood pressure in the rat. Clin Sci 2000;98:269-275

69. Pladys P, Lahaie I, Cambonie G, Thibault G, Le NL, Abran D. Nuyt AM. Role of brain and peripheral angiotensin $\mathrm{I}$ in hypertension and altered arterial baroreflex programmed during fetal life in rat. Pediatr Res 2004:55: 1042-1049 


\section{Chapter 10}

Samenvatting 
Uit epidemiologisch onderzoek blijkt dat mensen die geboren worden met een laag geboortegewicht een grotere kans hebben op het ontwikkelen van hypertensie en diabetes mellitus op volwassen leeftijd. Zo is, naarmate het geboortegewicht lager is, de bloeddruk hoger bij kinderen en volwassenen. De hypothese van foetale programmering probeert een verklaring te vinden voor dit fenomeen. Foetale programmering gaat uit van de hypothese dat schadelijke invloeden (hypoxie, maternale ziekteprocessen, groeivertraging) tijdens kritische ontwikkelingsperioden van de foetus tijdens het intra-uteriene leven in staat zijn orgaan- en regelsystemen te (re)programmeren, wat uiteindelijk op volwassen leeftijd kan leiden tot hypertensie en diabetes mellitus. Er zijn dierexperimentele aanwijzingen dat foetale groeivertraging leidt tot verandering in het bloeddrukregulatie systeem. Groeivertraging veroorzaakt sympatische hyperinnervatie van perifere zenuwvezels en verstoort zo de balans tussen het (ortho)sympatische en parasympatische zenuwstelsel. Verstoring van deze sympatovagale balans (verhoogde sympatische activiteit) kan leiden tot een verhoogde vaattonus en een verlies van bloeddrukregulatie met toegenomen variabiliteit in bloeddruk. Beide factoren spelen mogelijk een rol in de genese van hypertensie en orgaanschade.

De hypothese van foetale programmering vormde in beginsel aanleiding tot studie van het (korte-termijn) bloeddrukregulatie systeem (de baroreceptor reflex) bij de pasgeborene. Omdat nog weinig bekend is over ('het normale') functioneren van de baroreceptor reflex bij pasgeborenen en de perinatale invloeden hierop, werd exploratie van de baroreceptor reflex bij pasgeborenen met een bij de zwangerschapsduur passend geboortegewicht het eerste doel van het proefschrift. Dit betekende ondermeer dat onderzoek moest worden verricht naar a) het verwerven, opslaan en analyseren van hartslag- en bloeddruksignalen en b) observaties werden gedaan bij pasgeboren kinderen met een grote spreiding in zwangerschapsduur. Hierbij werd tevens gebruik gemaakt van medische interventies tijdens de intensieve zorg behandeling, namelijk toediening van atropine als farmacologische blokkeerder van de parasympaticus. In hoofdstuk 2, 3 en 4 wordt de werking van de baroreceptor reflex en de signaal analytische methodologie in algemene zin beschreven. In hoofdstuk 5 wordt ingegaan op het verwerven van continue bloeddrukwaarden op niet-invasieve wijze. In hoofdstuk 6,7 en 8 volgen drie klinische studies. In hoofdstuk 9 worden de bevindingen bediscussieerd en vooruitgekeken naar toekomstig onderzoek met betrekking tot foetale programmering van de baroreceptor reflex. 
Hoofdstuk 2 vormt de inleiding tot de baroreceptor reflex. Geregelde processen tonen fluctuaties rondom een streefwaarde. Deze fluctuaties worden veroorzaakt doordat het regelsysteem reageert op een verstoring. Het korte-termijn-regelsysteem voor bloeddruk wordt gevormd door de baroreceptor reflexboog. Deze bestaat uit sensoren, zenuwbanen naar en van het centraal autonome zenuwstelsel en eindorganen. De drukgevoelige sensoren in de arteriën van de grote circulatie worden aangeduid als baroreceptoren. Deze receptoren worden gevonden bij de aftakkingen van de grote vaten in de wand van de arcus aortae en in de wand van de sinus caroticus. In het dagelijkse leven worden verstoringen in bloeddruk voornamelijk veroorzaakt door houdingsveranderingen. De baroreceptor reflex voorkomt normaliter dat men niet duizelig wordt indien men van liggende positie naar staande positie verandert. De baroreceptoren nemen de bloeddrukverstoring waar en zenden via zenuwbanen informatie naar het autonome zenuwstelsel (regelcentrum), dat de bloeddrukverstoring vergelijkt met de streefwaarde. Vervolgens zenden sympatische en parasympatische zenuwbanen signalen naar het hart en de arteriolen, die bijsturing uitvoeren (hartslag verandering, vaattonus) met als doel de oorspronkelijke verstoring te niet te doen. Dit is een zogenaamd teruggekoppeld regulatiesysteem met negatieve feed-back. Fluctuaties in bloeddruk verstoren de hartslag met een zekere grootte (verstoring of gain) en met een zekere tijdsrelatie (fase). De baroreceptor gevoeligheid kan worden gezien als de klinische maat voor gain en wordt uitgedrukt in $\mathrm{ms} / \mathrm{mmHg}$.

Dierexperimenteel onderzoek laat zien dat er tijdens het foetale leven een functionele baroreceptor reflex aanwezig is. Of dit bij de mens ook zo is, is veel minder duidelijk.

In hoofdstuk 3 wordt ingegaan op de signaalanalyse van hartritme en bloeddruk variabiliteit en de betekenis hiervan voor de cardiovasculaire autonome regulatie. De regulatie van het cardiovasculaire systeem komt voor een zeer groot deel tot stand door het samenspel tussen sympatische en parasympatische activiteit van het autonome zenuwstelsel op de eindorganen. Dit samenspel tussen het sympatische en parasympatische systeem (de sympato-vagale balans) is dynamisch en zorgt voor variabiliteit in hartritme en bloeddruk. De variabiliteit in hartritme en bloeddruk kan worden toegeschreven aan een aantal basale fysiologische regelmechanismen. Zo worden zeer trage fluctuaties $(<0,04 \mathrm{~Hz}$, overeenkomend met minder dan 2 fluctuaties per minuut) geassocieerd met de thermoregulatie en hormonale processen. In dit proefschrift werden deze zeer trage fluctuaties buiten beschouwing gelaten. Laagfrequente fluctuaties (bandbreedte tussen 0,04 en $0,15 \mathrm{~Hz}$, overeenkomend met 29 fluctuaties per minuut) worden toegeschreven aan baroreceptor reflex activiteit. Aangenomen wordt dat de laagfrequente fluctuaties onder invloed staan van zowel de sympatische als de parasympatische activiteit. Hoogfrequente fluctuaties zijn 
geassocieerd aan de ademhaling en worden vrijwel uitsluitend gemoduleerd door parasympatische zenuwactiviteit. De aanzienlijke hogere hart- en ademhalingsfrequentie bij de pasgeborene noodzaakt tot een andere definiëring van de ademhalingsband dan de definiëring die wordt gebruikt bij de volwassene. In dit proefschrift is voor definiëring van de hoogfrequente ademhalingsband gebruik gemaakt van (1) de individuele ademfrequentie van het kind met de p-10 en p-90 waarde als grenzen van de hoogfrequente band of (2) van een vooraf vastgestelde hoogfrequente band tussen 0,4 en $1,5 \mathrm{~Hz}$, overeenkomend met de grenzen van ademhaling bij preterm geboren kinderen.

Spectraal analyse (Fourier analyse) is een techniek die een (fysiologisch) signaal kan ontleden in verschillende sinusfuncties. Spectraal analyse toont hiermee de relatieve bijdrage van verschillende fluctuaties als functie van de frequentie. Spectraal analyse van hartritme variabiliteit toont (idealiter) een laagfrequente piek rondom $0,1 \mathrm{~Hz}$ (baroreceptor reflex activiteit, onder sympatische en parasympatische controle) en een hoogfrequente piek rondom de ademhalingfrequentie (respiratoire sinus aritmie, onder parasympatische controle). De verhouding tussen laagfrequente tot hoogfrequente spectraal energie (de zgn. LF/HF ratio) wordt door veel onderzoekers als een maat gezien voor de sympato-vagale balans bij volwassenen. Kruisspectraal analyse van de laagfrequente $(0,04-0,15 \mathrm{~Hz})$ bloeddruk en hartritme variabiliteit geeft informatie over de onderlinge samenhang in grootte (verstoring of gain) en tijd (fase) van de baroreceptor reflex. De verstoring of gain wordt ook wel baroreceptor gevoeligheid genoemd. In het proefschrift worden twee kenmerken van de baroreceptor reflex (de baroreceptor gevoeligheid en de tijdrelatie) bepaald door deze kruisspectrale analyse van de laagfrequente fluctuaties in bloeddruk en hartslag.

In hoofdstuk 4 worden twee signaal analyse methoden vergeleken: een klassieke Fourier methode en een aangepaste filteringmethode. Een belangrijke beperking in de toepassing van Fourier analyse technieken is gelegen in de aanname van signaal stabiliteit. Prematuur geboren kinderen vertonen echter een snelle wisseling in slaap- en waakstadium. Als gevolg hiervan treden veranderingen op in hartritme- en bloeddruk variabiliteit en dus in spectrale energie. Enerzijds is een langere periode noodzakelijk voor betrouwbare schatting van (laagfrequente) fluctuaties, anderzijds wordt de tijd begrensd door de sterk wisselende fysiologische condities van het kind. Er zal dus een compromis moeten worden gevonden tussen een adequate resolutie in tijd en frequentie. Bij de klassieke Fourier methode wordt gebruik gemaakt van segmenten van 64 seconden lang. Met een aangepaste filtering techniek is het mogelijk deze periode te verkorten tot 42 seconden zonder verlies van resolutie in frequentie. 
Omdat alleen bij kritisch zieke pasgeborenen intra-arteriële navelkatheters worden gebruikt tijdens de intensieve zorg fase, werd in hoofdstuk 5 onderzocht of (slag-opslag) bloeddrukwaarden op een niet-invasieve manier betrouwbaar gemeten kon worden bij stabiele pasgeborenen. Bij volwassenen is het namelijk mogelijk op een niet-invasieve manier bloeddruk te meten door middel van een Finapres apparaat. Finapres staat voor "FINger Arterial PRESsure" en is door TNO (Biomedical instrumentation, Amsterdam, Nederland) ontwikkeld. Finapres meet continu op nietinvasieve wijze de bloeddruk aan de vinger en produceert een golfvorm die grote overeenkomsten vertoont met de perifere arteriële golf. Het principe van deze techniek is gebaseerd op servoplethysmometrie, gebruik makend van een "volume-clamp"techniek. Een - in de opblaasbare vingercuff ingebouwde -infrarode cel/sensor meet het bloedvolume van de vinger (plethysmografie). Veranderingen in bloedvolume worden opgevangen door continue aanpassingen van de cuffdruk door middel van een snelwerkend electropneumatisch systeem. Een gecomputeriseerd feedbacksysteem compenseert continu de intra-arteriële druk zodanig dat het drukverval over de arteriële wand (de transmurale druk) nul is. Bij een transmurale druk van nul wordt aangenomen dat de cuffdruk gelijk is aan de intra-arteriële druk. De cuff van de Finapres wordt normaliter aangebracht om de middelste phalanx van de middel- of ringvinger. Omdat er geen maat voorhanden is voor een baby vinger werd de kleinste (volwassen maat) cuff om de pols van het kind bevestigd. De hiermee verkregen waarden werden vergeleken met intra-arteriële bloeddrukwaarden bij pasgeborenen die ten behoeve van de intensieve zorg waren gekatheteriseerd. Uit de resultaten bleek dat de volwassen vinger cuff een goede pasvorm had voor de pols van prematuur geboren kinderen met een gewicht tussen de 1000 en 2500 gram. De absolute waarden van de bloeddruk gemeten met de Finapres weken aanzienlijk af van de intra-arterieel gemeten bloeddruk. De Finapres gaf echter goed vergelijkbare resultaten ten opzichte van de intra-arteriële metingen wat betreft het meten van veranderingen in bloeddruk. Dit biedt perspectief om de baroreceptor reflex gevoeligheid (verandering hartslag t.o.v. bloeddruk, eenheid: $\mathrm{ms} / \mathrm{mmHg}$ ) betrouwbaar op niet-invasieve wijze te meten tijdens houdingsveranderingen bij pasgeborenen.

In hoofdstuk 6 wordt verslag gedaan van een studie bij 10 te vroeg geboren kinderen (zwangerschapsduur: 27 - 34 weken), bij wie in de eerste levensweek de baroreceptor reflex functie werd bestudeerd door middel van (kruis)spectraal analyse van spontane hartritme- en bloeddrukfluctuaties. Hartritme (gemiddeld 140 hartslagen per minuut) en arteriële systolische bloeddruk (gemiddeld $59 \mathrm{mmHg}$ ) toonden langzame fluctuaties (met een frequentie van ongeveer $0,1 \mathrm{~Hz}$ en een bloeddruk amplitudo van $2-4 \mathrm{mmHg}$ ) en snelle fluctuaties (rond de ademhalingsfrequentie van $0,8 \mathrm{~Hz}$ met een bloeddruk 
amplitudo van $1 \mathrm{mmHg}$ ) bij klinisch stabiele prematuur geboren kinderen in het rustige slaap stadium. Omdat variabiliteit voornamelijk aanwezig was in de laagfrequente band $(0,03-0,15 \mathrm{~Hz})$ resulteerde dit in relatief hoge LF/HF ratio's. De baroreceptor gevoeligheid (gain) tussen de laagfrequente bloeddruk en hartslag fluctuaties bedroeg ongeveer $4 \mathrm{~ms} / \mathrm{mmHg}$, wat geringer is dan bij volwassenen $(20-30 \mathrm{~ms} / \mathrm{mmHg})$. De laagfrequente fluctuaties in bloeddruk werden gevolgd door fluctuaties in hartritme met een latentie tijd van ongeveer 4 seconden, wat trager is dan bij volwassenen ( 2 seconden). De baroreceptor reflex bij stabiele prematuur geboren kinderen tonen dus verschillen met volwassenen. De verhoogde $\mathrm{LF} / \mathrm{HF}$ ratio suggereert dat bij prematuur geboren kinderen sprake is van verhoogde sympatische activiteit en/of een onrijp parasympatisch systeem. Uit later onderzoek (hoofdstuk 7 en 8) zal echter blijken dat de toepassing van de LF/HF ratio bij prematuur geboren kinderen beperkt is.

In hoofdstuk 7 wordt een studie beschreven bij te vroeg geboren kinderen (zwangerschapsduur 26 tot 32 weken) in de acute ziekte fase direct na de geboorte, bij wie atropine is toegediend als premedicament van intubatie. Atropine verhoogde de hartfrequentie en verlaagde voornamelijk de laagfrequente (samenhangend met de baroreceptor reflex) variabiliteit in hartritme. In tegenstelling tot bij volwassenen nam de LF/HF ratio af na atropine. Het is mogelijk dat bij prematuur geboren kinderen hoogfrequente hartritme variabiliteit niet uitsluitend door de parasympaticus wordt gemoduleerd. Andere factoren (bijv. directe mechanische koppeling tussen thoraxbewegingen en atrium) zouden zelfs belangrijker kunnen zijn voor de inductie van hoogfrequente hartritme variabiliteit. Atropine had geen effect op de bloeddruk variabiliteit. Atropine verminderde significant de baroreceptor gevoeligheid. Samenvattend toont deze studie aan dat a) de parasympaticus een zeer belangrijke modulator is van de baroreceptor reflex bij te vroeg geboren kinderen; en b) de LF/HF ratio geen bruikbare maat lijkt te zijn voor de beschrijving van de sympato-vagale balans bij prematuur geboren kinderen.

Hoofdstuk 8 toont de invloed van de zwangerschapsduur op de baroreceptor reflex functie. Met het toenemen van de zwangerschapsduur daalt de hartslag; de hartritme variabiliteit neemt echter in belangrijke mate toe. Met het toenemen van de zwangerschapsduur stijgt de bloeddruk en neemt de bloeddruk variabiliteit bij voldragen kinderen gering af. De baroreceptor gevoeligheid neemt toe met de zwangerschapsduur en is gecorreleerd met de toename in hartritme variabiliteit. Zo hebben zeer vroeg geboren kinderen (28-32 zwangerschapsweken) een baroreceptor gevoeligheid van $4 \mathrm{~ms} / \mathrm{mmHg}$ en voldragen kinderen van $15 \mathrm{~ms} / \mathrm{mmHg}$. Dit betekent dat te vroeg geboren kinderen minder goed laagfrequente fluctuaties kunnen opvangen 
door aanpassing in hartritme. M.a.w. de baroreceptor reflex is minder goed ontwikkeld bij te vroeg geboren kinderen. Verdere rijping vindt plaats met het vorderen van de zwangerschapsduur en wordt gemoduleerd door parasympatische rijping.

In hoofdstuk 9 worden de belangrijkste conclusies van het proefschrift samengevat. Door middel van (kruis)spectrale analyse van hartslag en bloeddruksignalen kan inzicht verkregen worden in de korte termijn bloeddrukregulatie van de pasgeborene. De baroreceptor reflex is ook al bij extreem te vroeg geboren kinderen ( 27 weken zwangerschapsduur) aantoonbaar. De baroreceptor reflex gevoeligheid (maat voor de werking) is bij te vroeg geboren kinderen aanzienlijk kleiner dan bij op tijd geboren kinderen. De baroreceptor reflex wordt voornamelijk door het parasympatische deel van het autonome zenuwstelsel gemoduleerd.

Toekomstig onderzoek naar het effect van perinatale invloeden op (verstoring van) de cardiovasculaire autonome regulatie is nu de volgende stap. De effecten van foetale groeivertraging op de functie van de baroreceptor reflex na de geboorte zijn niet bekend bij de mens. Interessant zou zijn om na te gaan of de foetale effecten van groeivertraging zoals die gezien worden bij dierexperimenten (verstoring van de sympato-vagale balans; verlies van bloeddruk regulatie; toename van bloeddruk variabiliteit) kunnen worden gerepliceerd bij de mens. Uit dit proefschrift is echter gebleken dat de zgn. LF/HF ratio bij te vroeg geboren kinderen geen bruikbare maat is voor het schatten van de sympato-vagale balans. Als alternatieve methoden zou men gebruik kunnen maken van atropine toediening (blokkeerder van de parasympaticus en leidend tot uitsluitend sympatische (rest)activiteit) of het uitvoeren van snelle houdingsveranderingen door middel van zgn. kiepproeven (geeft een krachtige stimulatie van sympatische activiteit). Na atropine toediening zou men hartritme en bloeddruk variabiliteit en baroreceptor reflex gevoeligheid kunnen vergelijken tussen pasgeborenen met en zonder groeivertraging. Vergelijkbaar kan men het effect van kiepproeven op de bloeddruk regulatie bestuderen bij pasgeborenen met en zonder groeivertraging. Het gebruik maken van niet-invasieve methoden van continue bloeddrukmeting (Finometer) biedt hierbij grote voordelen. 



\section{Appendix}

A: Constructing a heart rate and blood pressure variability analysis

Andriessen P, Janssen RWJ, de Beer NAM

B: Feasibility of continuous noninvasive blood pressure measurement in infants

O. Schraa, P. Andriessen, B. Smit, W. van den Bosch-Ruis, M. van Koppen, J.J. Settels

C: Passive head-up tilting in neonates

P. Andriessen, B. Vermeulen, P. Baldussu 


\section{Appendix A}

\section{Analyzing heart rate and blood pressure variability}

The consecutive steps in analyzing variability from the ECG or beat-to-beat arterial blood pressure to auto and cross spectra are shown in Figure 1.

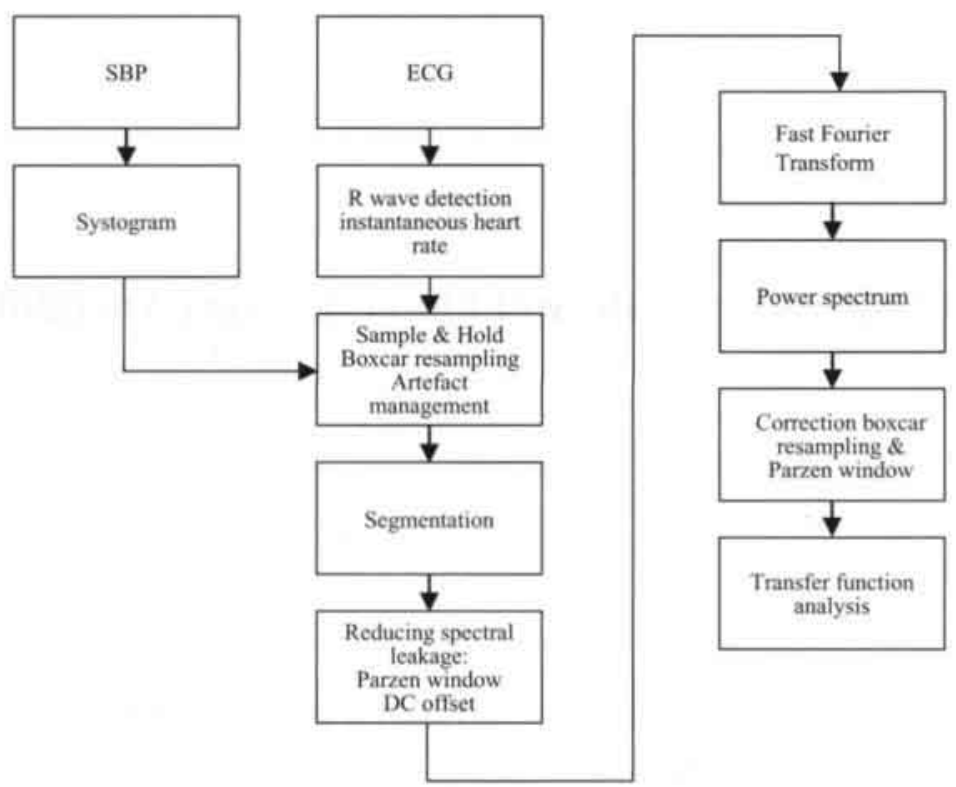

Figure 1. Arterial blood pressure (ABP), electrocardiogram (ECG), direct current (DC).

\section{Detection of $\mathbf{R}$ wave and arterial systolic blood pressure}

The sample frequency was $512 \mathrm{~Hz}$ for the surface electrocardiogram (ECG) signals (exception chapter 6: $400 \mathrm{~Hz}$ ) and $128 \mathrm{~Hz}$ for the arterial blood pressure (chapter $6: 100 \mathrm{~Hz}$ ). From the ECG the consecutive R waves are detected by an appropriate R top detection algorithm. The sampling error of $\mathrm{R}$ top detection (half the sampling interval, i.e. $0.98 \mathrm{~ms}$ ) was optimized using a second-order polynomial fit, resulting in an accuracy of $\mathrm{R}$ top detection of $0.16 \mathrm{~ms}$. The reciprocal value of the $\mathrm{R}-\mathrm{R}$ interval corresponds to the instantaneous heart rate. Thus, $\mathrm{R}$ waves were detected from the ECG and an unevenly spaced R-R interval sequence was created. Likewise, systolic blood pressure was identified from peak detection of the beat-to-beat arterial blood pressure signal, resulting in an unevenly spaced "systogram". Variability analysis was performed 
on 192-s-long segments, being a compromise between signal stability, duration of quiet sleep state and demands of sufficient duration.

\section{"Sample \& hold" procedure}

The original sampled data results in a set of non-equidistant R-R-intervals or systogram. Because of its non-equidistant nature these data points need to be resampled before further processing. Resampling is achieved by a "sample \& hold" procedure: i.e., the instantaneous heart rate at a certain time is taken equal to the most recent determined value. The resulting signal is constant for a certain interval and then abruptly jumps to another level where it maintains until the next "jump". Figure 2 shows the determined heart rates for a certain segment. Consider the $\mathrm{n}^{\text {th }}$ heart cycle. The following two intervals are defined:

$2 \Delta_{s}=t_{n}-t_{n-1}$

and

$2 \Delta_{s}^{\prime}=t_{n+1}-t_{n}$

Note that $2 \Delta_{s}$ is smaller than $2 \Delta_{s}^{\prime}$ because the R-R-value at $t_{n}$ is larger than the R-R-value at $t_{n+1}$.

In the "sample \& hold" procedure the interval in which the heart rate is said to be constant is defined as:

$t_{n}-\Delta_{s}<t \leq t_{n}+\Delta_{s}^{\prime}$

\section{Boxcar resampling}

Mathematically, the sample \& hold procedure is equivalent to convoluting to series of heart rate values with a boxcar window. The boxcar window is defined as:

$$
\begin{aligned}
b c(t) & =0 & & |t|>\frac{2}{f_{s}} \\
& =\frac{2}{f_{s}} & & |t| \leq \frac{2}{f_{s}}
\end{aligned}
$$




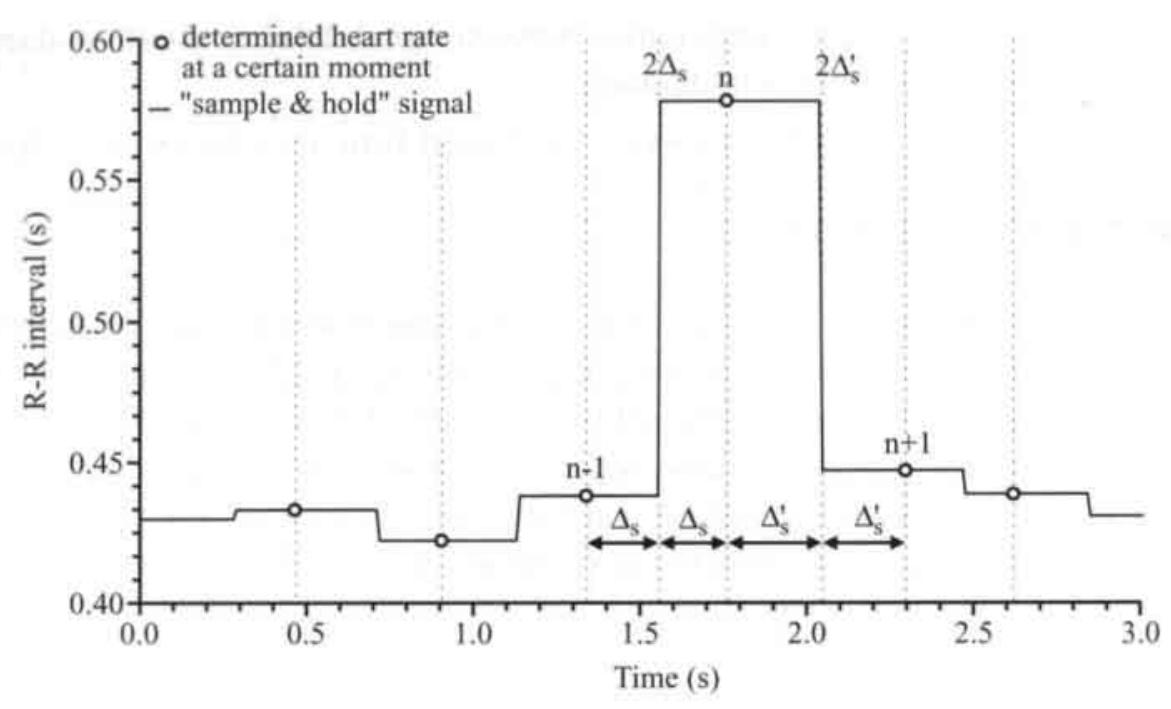

Figure 2. Illustration of the "sample \& hold" technique.

wherein $b c(t)$ denotes the boxcar and $f_{s}$ denotes the sample frequency. Because this convolution introduces discontinuities leading to erroneous high frequency components in the signal, the spectrum of the resulting signal has to be corrected for this convolution, as will be described in the section 'Boxcar resample correction'.

Empirically the resample rate is chosen to be $4 \mathrm{~Hz}$, i.e. the lowest power of 2 above the maximal heart rate in neonates ${ }^{1,2}$.

After applying the "sample \& hold" procedure to the heart rate series, the resulting signal will be resampled at $f_{s}=4 \mathrm{~Hz}$.

$y[n]=\sum_{k=-\infty}^{\infty} h[k] x[n-k]$

The "systogram" was converted into an equidistantly spaced time series using the same resampling method as used for the R-R interval time series.

The equidistant data was visually verified for ectopic beats, missing data or baseline drift. The data selection during the quiet sleep state resulted in $\mathrm{R}-\mathrm{R}$ interval artifact free segments. In the atropine study (chapter 7) an algorithm identified R-R artifacts (R-R interval $>500 \mathrm{~ms}$ or $<200 \mathrm{~ms}$ ) and missing R-R intervals were linearly interpolated. 


\section{Segmentation}

Each 192-s-long segment of preprocessed evenly spaced R-R interval and systolic blood pressure series was subdivided (segmentation) in 5 half-overlapping 64-s (256 points) segments. Using 64-s segments (resulting in a spectral resolution of $0.016 \mathrm{~Hz}$ ) and 5 half-overlapping segments power estimation above $0.04 \mathrm{~Hz}$ was considered to be acceptable (see section: Accuracy of measurements).

\section{Reducing spectral leakage}

The spreading out of spectral energy across several frequency bins is called spectral leakage.

Spectral leakage affects any frequency component of a signal which does not exactly coincide with a frequency bin (Equation A.6) and is caused by the truncation of a signal.

$f \neq n \Delta f ; \quad n= \pm 0,1,2,3 \ldots$

Figure 3 shows the truncation of a signal $\mathrm{s}(\mathrm{t})$. This can be interpreted as multiplying $\mathrm{s}(\mathrm{t})$ by a rectangular window function $\mathrm{h}(\mathrm{t})$. The Fourier transform (see next section) expands this truncated segment with a period $\mathrm{T}$. Signal $\mathrm{s}(\mathrm{t})$ does not match the above criterion, causing discontinuities at the edges of adjacent sections. These discontinuities created by the pattern mismatch give rise to the spurious components in the spectrum of the signal. This causes a particular frequency component to appear not as a single sharp line concentrated in just one frequency bin but as a spread of frequencies, roughly
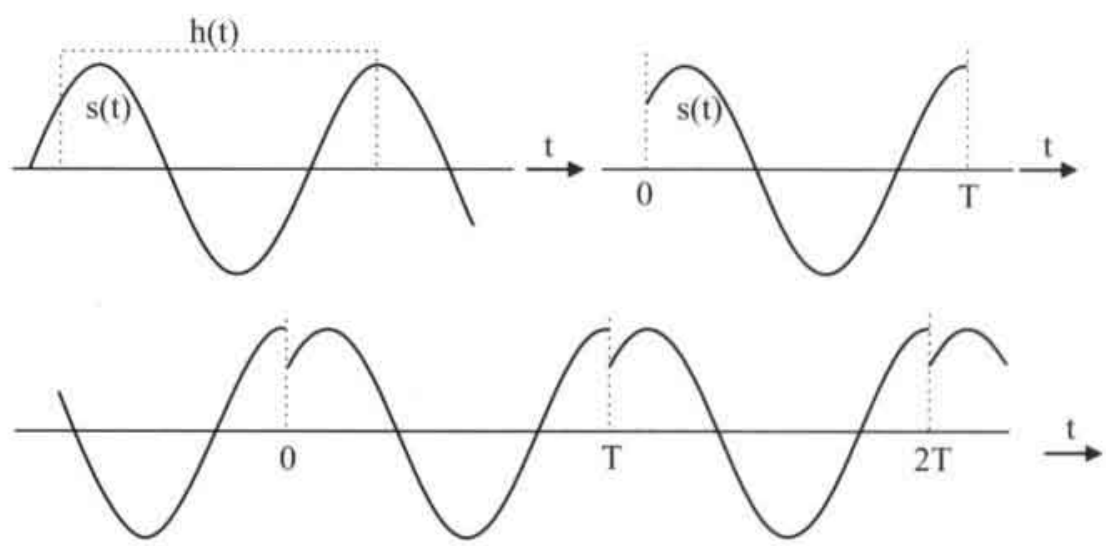

Figure 3. Spectral Leakage introduced by discontinuities at the edges of the signal's periodic expansion. 
centered around where the frequency component should be located, somewhere between the two nearest frequency bins on either side.

Spectral leakage can also be reduced by applying a tapered window function instead of a rectangular window function. Rather than starting and stopping abruptly, the signal now fades in and out towards zero at either end. This reduces the effect of the discontinuities where the mismatched sections of the signal join and hence the amount of leakage. A disadvantages of a tapered window function is that lines in the spectrum become broadened, making it difficult to distinguish separate frequency components and therefore effectively reducing the spectral resolution. A number of tapered window functions have been devised. Some are more effective in reducing spectral leakage, at the expense of loss of spectral detail, while others try to achieve a compromise between these conflicting requirements. In the presented studies a triangle shaped Parzen window is used ${ }^{3}$.

Likewise, to reduce spectral leakage of very low frequency $(0 \mathrm{~Hz})$, the mean R-R interval was calculated and subtracted from all data points to remove the direct current component.

\section{Fourier transform}

The Fourier transform performs the conversion of a function in time domain to the frequency domain and visa versa. The Fourier transform of a continuous infinite signal $s(t)$ is given by

$S(f)=\int_{-\infty}^{\infty} s(t) e^{-2 \pi i f t} d t$

For a signal with a finite length T Equation A.7 becomes:

$S(f)=\int_{0}^{T} s(t) e^{-2 \pi i f t} d t$

However, sampled signals require a discrete version of Equation A.8. Consider a signal $s(t)$ with a finite length of $\mathrm{T}$ seconds which is sampled with a sample rate $f_{s}$. This results in the discrete function

$s_{k}=s\left(t_{k}\right), \quad t_{k}=k \Delta t, \quad k=0,1,2, \ldots N-1$

with sample interval 
$\Delta t=\frac{1}{f_{s}}=\frac{T}{N}$

and $N$ denotes the total number of samples.

The digital Fourier transform of a signal consisting of $N$ (even) samples results in a Fourier spectrum consisting of $N$ independent values for $f$ :

$f_{n}=n \Delta f, \quad n=-\frac{N}{2}+1, \ldots, \frac{N}{2}$

with a frequency resolution of

$\Delta f=f_{n+1}-f_{n}=\frac{f_{s}}{N}=\frac{1}{T}$

This implies that

$-\frac{f_{s}}{2}<f_{n} \leq \frac{f_{s}}{2}$

Using Equations A.9 - A.12 the finite continuous Fourier transform (Equation A.8) becomes

$S(n \Delta f)=\frac{T}{N} \sum_{k=0}^{N-1} s(k \Delta t) e^{\frac{-2 \pi i k n}{N}}, \quad n=-\frac{N}{2}+1, \ldots, \frac{N}{2}, \quad k=0,1,2, \ldots, N-1$

The digital Fourier transform is independent of the normalization with respect to time and frequency. Therefore Equation A.14 can be expressed as

$S\left(f_{n}\right)=S_{n}=\frac{1}{N} \sum_{k=0}^{N-1} s_{k} e^{\frac{-2 \pi i k n}{N}}, \quad n=-\frac{N}{2}+1, \ldots, \frac{N}{2}, \quad k=0,1,2, \ldots, N-1$

\section{Aliasing and the Nyquist criterion}

The Nyquist criterion requires a signal to be sampled at least twice as often as its highest frequency to enable correct reconstruction of the signal. For a bandwidthlimited signal $-f_{c} \leq f \leq f_{c}$ this means that

$f_{s} \geq 2 f_{c}$

When the signal contains frequency components outside $\left[-f_{c}, f_{c}\right]$, the discrete spectrum 
of the signal will, due to the sampling, show overlay with its periodic expansion. This is illustrated in Figure 4 shows the spectrum of a continuous signal (left panel) as well as the spectrum of this same signal sampled at a frequency which does not meet the Nyquist criterion (right panel). The effect in the signal caused by the overlapping parts of the spectrum in the right graph is called 'aliasing'.
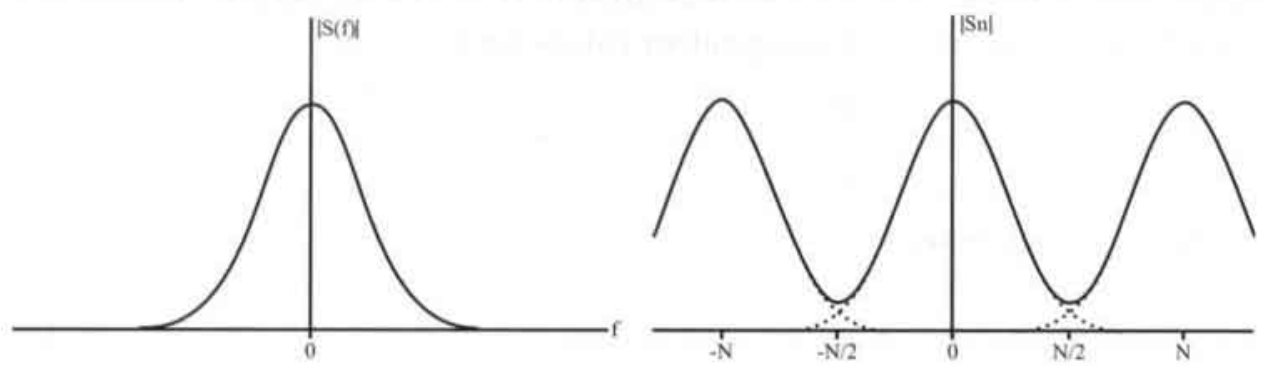

Figure 4. Continuous spectrum without aliasing (left panel) and the corresponding discrete spectrum with aliasing (right panel).

\section{Parseval's theorem and the power spectrum}

Parseval's theorem states that the Fourier transform conserves energy, or

$\int|s(t)|^{2} d t=\int|S(f)|^{2} d f$

This implies that in order to obtain a signal's energy, the square of the Fourier transform $|S(f)|^{2}$ can be used. This is called the power spectrum. Because we are only interested in frequencies from 0 to $1 / 2 f_{s}$ the single-sided power spectrum is used, neglecting the negative frequency components. For real signals, as we are using here, the power spectrum is always symmetrical around zero, and the negative frequencies do not give any additional information. This implies that, when working with the single-sided spectrum, the amplitude should be doubled when taking the integral as in Parseval's theorem.

\section{Boxcar resample correction}

As stated earlier the heart rate signals are convoluted with a boxcar window. Depending on the power of the used boxcar, this causes a gain or loss in the spectral power of the heart rate signals. For a normalized boxcar window, the change in spectral power will 
constitute an overemphasis of high frequencies. To compensate for this change of power, the Fourier spectrum of the heart rate signals has to be divided by the spectrum of the boxcar function (Equation A.4). After all, convolution (Equation A.5) in the timedomain corresponds to multiplication in the frequency domain.

The Fourier transform of a boxcar (or any other rectangular) window is given by

$H(f)=T \frac{\sin (\pi T f)}{\pi T f} \equiv T \operatorname{sinc}(\pi T F)$

\section{Parzen window correction}

To reduce the effect of spectral leakage, a Parzen window was applied. As can be seen from Figure 5, this window decreases the spectral power of the original signal and therefore the signal should be corrected for this procedure. This is achieved by dividing the power spectrum of heart rate variability signal by the average power of the Parzen window. Note that this correction is not necessary when a normalized Parzen window (with signal power 1) is applied.
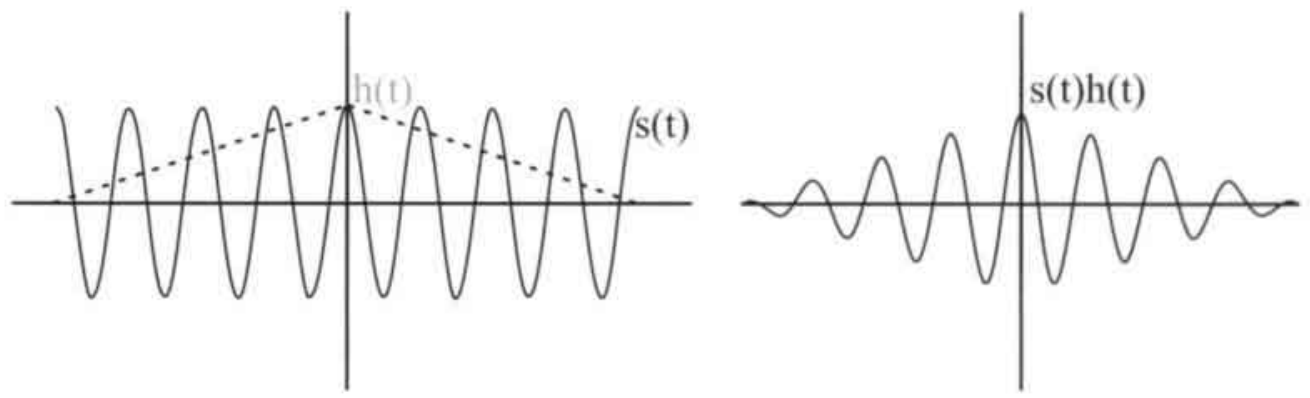

Figure 5. Left panel: Sinusoidal signal $s(t)$ and Parzen window h(t). Right panel: Parzen windowed signal $s(t) \cdot h(t)$.

\section{Transfer function analysis}

The amount of linear coupling between two variables in the frequency domain is usually expressed by the coherence function ${ }^{4}$. The coherence of time series $x(t)$ and $y(t)$ measures the variance of $y$ linearly predictable from $x$ at each frequency $f$. Coherence serves as the frequency domain analog of $r^{2}$, the coefficient of determination. Like $r^{2}$, its value lies between 0 and 1 , with values near 1 indicating a strong linear relation 
between the two series. Coherence is defined as the ratio of the squared covariance of the two time series to the product of their individual variances.

If $S_{x x}$ and $S_{y y}$ denote the auto spectra of $x$ and $y$, and $S_{x y}$ denotes their cross spectrum, then coherence $\gamma^{2}(f)$ is given at each frequency $f$ by

$$
\gamma^{2}(f)=\frac{\left|S_{x y}(f)\right|^{2}}{S_{x x}(f) S_{y y}(f)}
$$

The coherence level indicates the strength of the linear association and provides an estimate of variability in phase between two variables (e.g. blood pressure and R-R interval series). After the work of de Boer et al, it has become common to claim a significant linear relation between two cardiovascular time series when coherence values exceed 0.5 . The level of 0.5 indicates a relation between two signals based on $50 \%$ shared variance.

The transfer function gain and phase between blood pressure and R-R interval series were estimated from the auto- and cross-spectral density functions using a fast Fourier technique 5 .

If $x(t)$ denotes the input variable, $y(t)$ the output variable, $S_{x x}$ the autospectra of $x$ and $S_{x y}$ the cross-correlation spectrum, then the transfer function $H(f)$ is given by

$H(f)=\frac{S_{x y}(f)}{S_{x}(f)}=|H(f)| e^{-i \phi(f)}$

wherein $|H(f)|$ represents the transfer gain and $\phi(f)$ the transfer phase between the variable $x$ and $y$.

The transfer gain $|H(f)|$ is expressed in $\mathrm{s} / \mathrm{mmHg}$ as function of $f$. If $\tau(f)$ denotes time delay (s) as function of $f$, then the relation between $\phi(f)$ and $\tau(f)$ is given by

$$
\tau(f)=\frac{1}{360} \cdot \frac{\phi(f)}{f}
$$

with a periodicity of $360^{\circ}+n$ or $360^{\circ}-n$. 


\section{Accuracy of measurements}

\section{Spectral power estimation}

The standard error of the value per frequency bin is $100 \%$ of the calculated power spectral density6.7. Segmentation of the signal into half-overlapping segments will reduce the error in spectral power. Therefore the 192-s long segments were divided into 5 half-overlapping 64-s ( 256 points) segments.

If $n_{d}$ denotes the number of overlapping segments than the relative error in power estimation is given by

$\varepsilon_{r}=\frac{1}{2} \cdot \sqrt{\frac{9 n_{d}-1}{8 n_{d}^{2}}}$

(dimensionless)

Averaging five power spectra lead to a relative error of mean spectral power of $23 \%$ per frequency bin.

\section{Transfer function estimation}

If $\gamma^{2} x y\left(f_{n}\right)$ denotes the coherence of the transfer function between $x(t)$ and $y(t)$ at frequency $f_{n}$ and $n_{e}$ denotes the amount of overlapping segments, the relative error $\left(\varepsilon_{r}\right)$ of the transfer gain is given by ${ }^{8}$

$\varepsilon_{r}\left|H\left(f_{n}\right)\right|=\frac{\left[1-\gamma_{x y}^{2}\left(f_{n}\right)\right]^{\frac{1}{2}}}{\left|\gamma_{x y}\left(f_{n}\right)\right| \sqrt{2 n_{e}}} \quad$ (dimensionless)

The absolute error $\phi\left(f_{n}\right)$ of the transfer phase is given by

$\sigma\left[\phi\left(f_{n}\right)\right]=\frac{\left[1-\gamma_{x y}^{2}\left(f_{n}\right)\right]^{\frac{1}{2}}}{\left|\gamma_{x y}\left(f_{n}\right)\right| \sqrt{2 n_{e}}} \quad(\mathrm{rad})$

Although the right side of the equation is the same, the interpretation is different for transfer gain and phase. The table shows the relative error for transfer gain and absolute error for transfer phase - using 5 half-overlapping 64-s segments - as function of coherence values. 
Table: The relative error of the transfer gain $\left(\varepsilon_{r}\right)$ and absolute error $(\sigma)$ as function of coherence values

Coherence value $\varepsilon_{r}$ transfer gain $(\%)$ $\sigma$ transfer phase (degree)
0.5
32
18
0.6
26
15
0.7
21
12
0.8

\section{References}

1. Jaffe RS, Fung DL. Constructing a heart rate variability analysis system. J Clin Monit 1994;10:4558

2. de Jong W. Blood pressure variability in neonates: with a special focus on signal acquisition and signal processing. $\mathrm{PhD}$ Thesis 2000. Eindhoven University of Technology, Eindhoven, The Netherlands

3. Parzen E. Time series analysis papers. Holden-Day. San Fransisco, 1967

4. de Boer RW, Karemaker JM, Strackee J. Relationships between short-term blood pressure fluctuations and heart rate variability in resting subjects: a spectral approach. Med Biol Eng Comput $1985 ; 23 ; 352-358$

5 Bartels LW. A cross spectral analysis method between heart rate and blood pressure variability in neonates, Eindhoven, Technical University of Eindhoven, Master thesis, 1997.

6. Welch PD The use of fast Fourier transform for the estimation of power spectra: a method based on time averaging over short, modified periodograms. IEEE Trans Audio and Electroacoust 1967:15:7073

7 Bingham C. Godfrey MD, Tukey JW. Modern techniques of power spectrum estimation. IEEE Trans Audio and Electroacoust 1967:15:56-66

8. Bendat JS. Piersol AG. Random data, analysis and measurement procedures. John Wiley \& Sons, New York, 1986 


\section{Appendix B}

\section{Background \& Objectives}

The fetal origins hypothesis states that intrauterine growth retardation is strongly associated with hypertension in adults. Recent animal studies show hyperactivity of the sympathetic system in chronic hypoxic fetuses, which might be related to maladapted baroreflex function. Investigating autonomic cardiovascular regulation as early as possible therefore is important, with noninvasive continuous blood pressure a key parameter. Based on our previous finger arterial blood pressure technology (as implemented in Finapres and successors) we are interested to develop a system that measures blood pressure in infants.

\section{Design and Methods}

We investigated the feasibility of several prototype versions miniature finger cuffs (Figure 6) in 18 infants and children admitted to the (N)ICU's (Amsterdam and Veldhoven) who had intra-arterial blood pressure measurements for their clinical care. Gestational age ranged from 32 to 40 weeks and postnatal age ranged from 2 days to 1 year, weight ranged from 1.7 to $11 \mathrm{~kg}$. Experimental periods were limited to 30 minutes and included measurements of finger physiology of the artery around collapse. In addition, trial finger blood pressure recordings were made using the adult volume clamp set-point algorithms as implemented in Finapres, Portapres and Finometer.

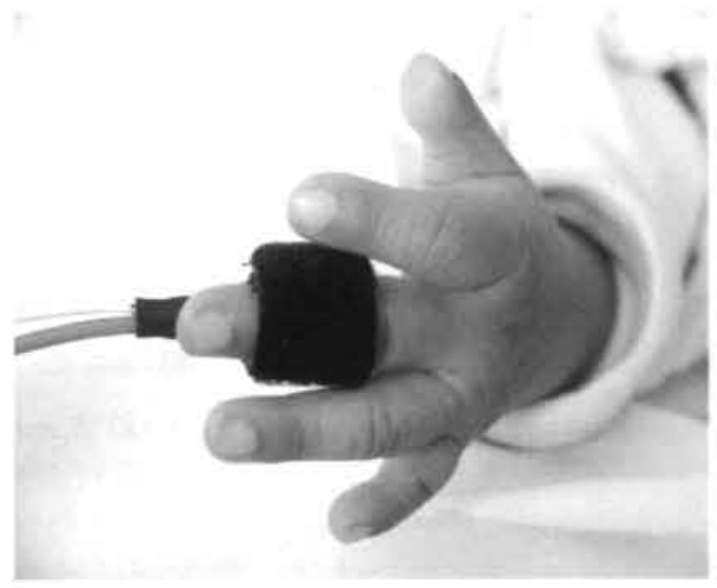

Figure 6. The baby finger cuff is placed around the middle finger. 


\section{Results}

The optical components and the inflatable bladder which must provide a uniform pressure transmission to the finger proved to be critical issues, and were modified several times in various prototype cuffs. As a related issue, the cuff application to the finger is a critical item. The adult volume clamp set-point algorithm did not find a proper value and sometimes showed erratic behaviour. Nevertheless, in 17 out of 18 subjects we were able to record waveforms with good resemblance to the intra-arterial waveforms.

\section{Conclusions}

The cuff design proved to be critical to obtain the data needed for the understanding of the physiology of newborn finger arteries near collapse under a volume clamp cuff and the differences with adult physiological relations. Since the set-point strategy for adults does not fit, these relations are essential to develop a new volume clamp set-point strategy. Our waveform data collected so far is an encouraging preliminary indicator of the ultimate feasibility.

\section{Reference}

Schraa O, Andriessen P, Smit B, van den Bosch-Ruis W, van Koppen M, Settels J. Feasibility of continuous noninvasive blood pressure measurements in infants. J Hypertens 2004;22 (suppl 2):S280 


\section{Appendix C}

Recently the Department of Medical Technology of Máxima Medical Center developed a tilting table for infants (Figure 7). A regular baby cot was adapted into a tilting cot which allowed smooth tilts of any degree between $0^{\circ}$ and $+60^{\circ}$.

The infant is placed in the supine position and a vacuum mattress was moulded around the baby by sucking out the air to prevent any sliding or movement during tilting. The baby is secured with straps around it and covered with blankets and a quilt.

In addition, a mobile acquisition system is developed and equipped with a neonatal monitor (past: Hewlett Packard monitor, type Merlin; present: General Electrics neonatal monitor), Finometer (TNO Institute of Applied Physics, Biomedical Instrumentation, Amsterdam) and a Compaq personal computer to measure ECG, blood pressure, thorax impedance and oxygen saturation.

In a pilot study of 12 healthy term infants, swift tilts between $0^{\circ}$ and $+60^{\circ}$ were achieved within $5 \mathrm{~s}$. The tilting procedure resulted in small motor movements and a temporarily change of the respiratory pattern. Most subjects remained asleep in the $+60^{\circ}$ position. In 10 subjects the heart rate increased after tilting without a change in blood pressure (oscillometric measurements every minute).

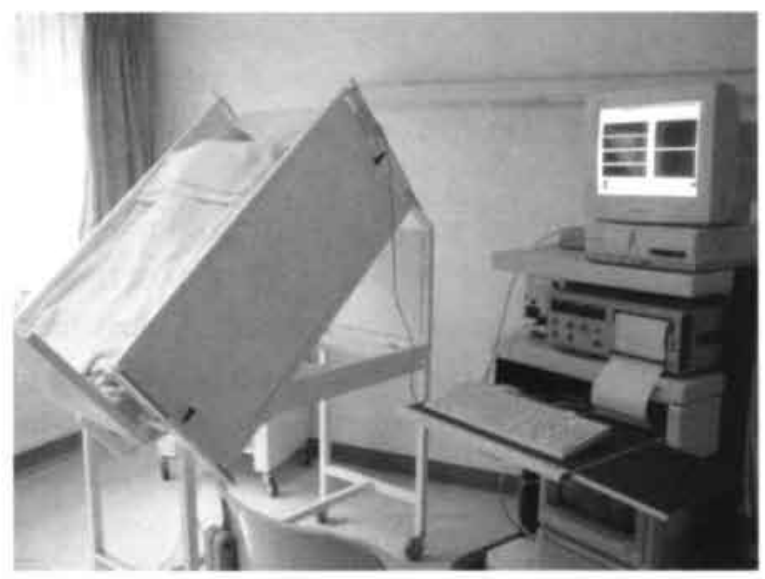

Figure 7. The tilting table for infants and data acquisition system are developed at Máxima Medical Center. 



\section{Dankwoord}

Allereerst: bedankt lieve kinderen, bij wie ik de klinische studies heb mogen verrichten. Tijdens jullie verblijf op de neonatologie afdeling werden hartslag-, ademhaling- en bloeddruksignalen gemeten en opgeslagen op een computer, en vormde zo de basis voor dit proefschrift.

Dit proefschrift is een gevolg van een intensief samenwerkingsverband tussen de afdelingen neonatologie en klinisch fysica van Máxima Medisch Centrum te Veldhoven, de faculteiten Elektrotechniek en Natuurkunde van de Technische Universiteit Eindhoven en de afdeling neonatologie van het Academisch Ziekenhuis Maastricht.

Mijn promotor prof. dr. C.E. Blanco, dank ik voor de plezierige en ontspannen wijze waarop hij mij bij het onderzoek begeleid heeft. Beste Carlos, eind 2000 ben ik bij je langs gekomen met de vraag dat ik graag onderzoek wilde doen in samenwerking met Maastricht. Jouw taxatie (Peter, waar is Veldhoven goed in?) en een wetenschappelijk relevante vraagstelling (Peter, wat lijkt je van foetale programmering?) leidde tot het onderzoeksprotocol BIRTH (Birth weight-In-Relation-To-Hypertension). Je hebt mij veel vrijheid gegeven in de uitvoering van het onderzoek. Zelfs verandering van het proefschrift onderwerp ('Birth weight' werd: 'Baroreceptor', en de relatie met 'Hypertension' wordt onderwerp van vervolgonderzoek) was geen probleem. Als de grote lijn maar duidelijk is. Het gaf (en geeft) mij veel plezier en voldoening om wetenschappelijk onderzoek te doen!

Mijn co-promotor, collega dr. Bambang Oetomo, dank ik voor de stimulerende opmerkingen tijdens het schrijven van de artikelen. Beste Sidarto, bedankt voor je enthousiasme.

De leden van de leescommissie dank ik voor hun tijd om mijn wetenschappelijke proeve van bekwaamheid te toetsen op kwaliteit.

Zeer veel dank gaat uit naar André Koolen, mijn collega kinderarts-neonatoloog, voor zijn bijdrage in de beginfase van het onderzoek. Beste André, zonder jouw visie en inspanning zou het nooit mogelijk zijn geworden dit proefschrift te schrijven! Bijna alle noodzakelijke voorwaarden voor acquisitie en data analyse zijn door jouw toedoen mogelijk gemaakt.

Ik dank mijn collega's van de vakgroep kindergeneeskunde voor hun interesse in het onderzoek. Ik wens Martin de Kleine, René van Gent en Jan Buijs veel succes in hun bijdrage de medische wetenschap te verrijken met kennis over neonatale follow-up, niet ontdekt astma bij schoolgaande kinderen en cerebrale beeldvorming bij asfyctische pasgeborenen. Ik wens de overige vakgroepleden veel succes in het lezen en doorgronden van al deze kennis. 
Ook dank ik het ziekenhuismanagement (Hans Brands en Martén van de Goor) dat zij geld en andere middelen wisten vrij te maken voor de uitvoering van wetenschappelijk onderzoek in een niet-academisch ziekenhuis.

Het Technologisch Onderzoek Perinatologie (TOP), een overlegstructuur binnen Máxima Medisch Centrum vormde het platform waar verschillende disciplines (technici, ingenieurs en medisch specialisten) elkaar ontmoetten. Van de overleggroep wil ik noemen: prof. dr. S.G. Oei, gynaecoloog Máxima Medisch Centrum en hoogleraar faculteit Biomedische Technologie, Technische Universiteit Eindhoven (voorzitter); prof. dr. P.F.F. Wijn, klinisch fysicus Máxima Medisch Centrum en hoogleraar faculteit Natuurkunde, Technische Universiteit Eindhoven; ir. R.C.M Berendsen en ir. C.H.L. Peters, klinisch fysici; ir. B. Vermeulen en ing. M. Schasfoort, medisch technologen Máxima Medisch Centrum en dr. ir. N.A.M. de Beer, post-doc fysica faculteit Elektrotechniek, Technische Universiteit Eindhoven. Beste Guid, jij hebt ervoor gezorgd dat het TOP overleg een regelmatig karakter kreeg. Beste Pieter, bedankt voor je visie in medisch technologische ondersteuning van de neonatale intensive care unit en de aanvoer van afstudeerders van de Technische Universiteit Eindhoven. Beste Ralph en Nicole, bedankt voor de inhoudelijke discussies en jullie bijdrage aan het proefschrift. Zonder jullie inbreng was het niet gelukt. Bedankt Barbara en Martijn, als het meetsysteem 'down' ging zorgden jullie voor een oplossing. Beste Chris, ik wens je succes in het (her)structureren van een stabiel acquisitie systeem. Bedankt Rick, voor je bijdrage in de Finapres studie. Bedankt Rogier, voor je bijdrage in het beschrijven van de wiskundige analyse ten behoeve van Appendix A. Veel dank ben ik ook verschuldigd aan dr. B.J.A. Janssen (afdeling Farmacologie \& Toxicologie van de Universiteit van Maastricht) voor zijn bijdrage aan de atropine studie. Beste Ben, ik hoop dat we in de toekomst de (soms verschillende) observaties bij dier- en humane studies dichter bij elkaar kunnen brengen. Ik heb veel van je geleerd.

Ik wil ir. O. Schraa (fysicus van TNO Biomedical Instrumentation, Amsterdam) bedanken voor de uitleg van de Finometer. Beste Olaf, ik hoop in de toekomst verder met je samen te werken op het gebied van het ontwikkelen van een baby 'finger cuff'. Succes Olaf met jouw boekje, houdt hierbij stelling 7 in gedachte.

Ik wil Henk en Lars Dinnissen bedanken voor het maken van de lay-out van het proefschrift. Beste Henk, het is leuk om te zien hoe een manuscript langzamerhand een echt boekje wordt. Je hebt hart voor de zaak.

Beide paranymfen, Hans van der Laan en Diederik van Romondt, en hun partners, wil ik bedanken voor de vriendschap en gezelligheid, en hun ideeën en hulp om de promotie tot een onvergetelijke dag te maken. 
Mijn ouders wil ik bedanken voor hun stimulatie tijdens mijn middelbare school loopbaan en hun materiële en immateriële hulp tijdens de medische studie.

Ik heb overigens geen proefschrift nodig om mijn vrouw en kinderen te bedanken. Ik dank elke dag al voor het voorrecht hen te kennen.

De grootste dank gaat uit naar de Maker van hemel en aarde. Onvoorstelbaar hoe de mens geschapen is. Zelfs een relatief eenvoudig systeem, zoals de baroreceptor reflex, is ingenieus en ondoorgrondelijk gemaakt: het elektronische zoekprogramma Alta Vista levert al 16000 'hits' op. 


\section{Curriculum Vitae}

De auteur van dit proefschrift werd op 30 juli 1962 geboren in Eindhoven. Nadat hij achtereenvolgens de MAVO, HAVO en Atheneum B doorlopen had werd in 1983 begonnen met de studie geneeskunde aan de Universiteit van Maastricht. In 1987 en 1989 behaalde hij achtereenvolgens het doctoraal- en artsexamen, beide voorzien van het predikaat "cum laude". De eerste beginselen in de neonatologie werden geleerd als arts-assistent-niet-in-opleiding in het Sint Radboud Ziekenhuis te Nijmegen. In 1991 werd begonnen met het academische deel van de opleiding tot kinderarts in het Sint Radboud Ziekenhuis te Nijmegen (opleider: prof. dr. R.C.A. Sengers). Het nietacademische deel van de opleiding werd vervolgd in het Sint Joseph Ziekenhuis te Veldhoven (opleiders: dr. E.J.P. Lommen, dr. W.E. Tjon A Ten). In 1996 werd hij ingeschreven in het medisch specialistenregister als kinderarts. Tijdens het laatste half jaar van de opleiding kindergeneeskunde werd tevens een start gemaakt met een fellowship neonatologie in het (toenmalige) Sint Joseph Ziekenhuis (18 maanden, opleider: M.J.K. de Kleine). Dit fellowship werd vervolgd met een academisch deel aan de Vrije Universiteit van Amsterdam (6 maanden, opleider: prof. dr. H.N. Lafeber). Gedurende twee jaar (1998-1999) was de auteur als kinderarts-neonatoloog werkzaam in de neonatale intensive care unit van de Vrije Universiteit van Amsterdam (hoofd: prof. dr. W.P.F. Fetter). Na een half jaar in het Sophia kinderziekenhuis te Rotterdam (hoofd: prof. dr. J.N. van den Anker) te hebben gewerkt, keerde de auteur in 1999 terug naar de neonatale intensive care unit van het Sint Joseph Ziekenhuis te Veldhoven. Het Sint Joseph Ziekenhuis werd - na de fusie met het Diaconessenhuis te Eindhoven omgedoopt tot Máxima Medisch Centrum. Het wetenschappelijk onderzoek ten behoeve van dit proefschrift werd geheel uitgevoerd in de neonatale intensive care unit van Máxima Medisch Centrum, locatie Veldhoven.

Tijdens de studie geneeskunde leerde de auteur zijn vrouw Petra kennen, met wie hij sinds 1987 getrouwd is. Samen hebben zij 5 kinderen: Floortje, Lisa, Anneloes, Bas en Nadine. 

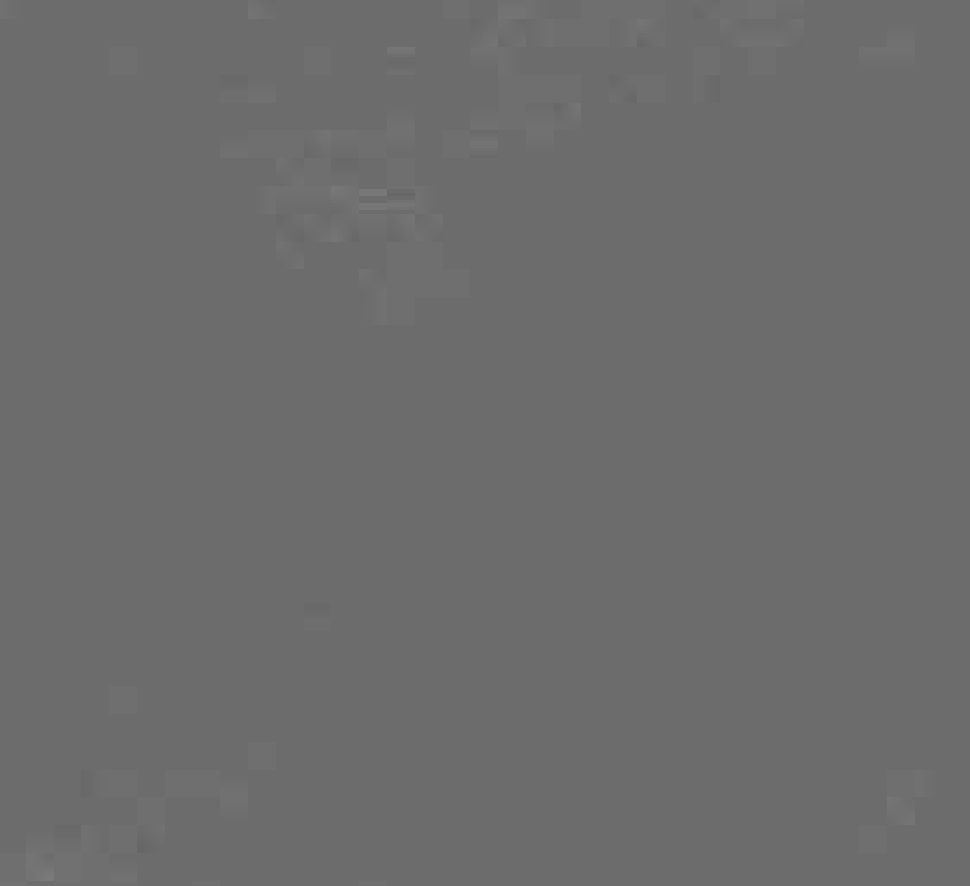

.

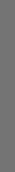

나난

1 II

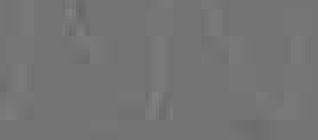
$3=$ $-\quad=1 \%$ 